\title{
Universiteit
}

Leiden

The Netherlands

\section{Computational approaches to dissociative chemisorption on metals: towards chemical accuracy}

Kroes, G.J.

\section{Citation}

Kroes, G. J. (2021). Computational approaches to dissociative chemisorption on metals: towards chemical accuracy. Physical Chemistry Chemical Physics, 23(15), 8962-9048. doi:10.1039/d1cp00044f

Version: $\quad$ Publisher's Version

License: $\quad$ Licensed under Article 25fa Copyright Act/Law (Amendment Taverne)

Downloaded from: https://hdl.handle.net/1887/3216927

Note: To cite this publication please use the final published version (if applicable). 
Check for updates

Cite this: Phys. Chem. Chem. Phys., 2021, 23, 8962

\section{Computational approaches to dissociative chemisorption on metals: towards chemical accuracy}

\author{
Geert-Jan Kroes (iD)
}

We review the state-of-the-art in the theory of dissociative chemisorption (DC) of small gas phase molecules on metal surfaces, which is important to modeling heterogeneous catalysis for practical reasons, and for achieving an understanding of the wealth of experimental information that exists for this topic, for fundamental reasons. We first give a quick overview of the experimental state of the field. Turning to the theory, we address the challenge that barrier heights $\left(E_{\mathrm{b}}\right.$, which are not observables) for DC on metals cannot yet be calculated with chemical accuracy, although embedded correlated wave function theory and diffusion Monte-Carlo are moving in this direction. For benchmarking, at present chemically accurate $E_{\mathrm{b}}$ can only be derived from dynamics calculations based on a semi-empirically derived density functional (DF), by computing a sticking curve and demonstrating that it is shifted from the curve measured in a supersonic beam experiment by no more than $1 \mathrm{kcal} \mathrm{mol}^{-1}$. The approach capable of delivering this accuracy is called the specific reaction parameter (SRP) approach to density functional theory (DFT). SRP-DFT relies on DFT and on dynamics calculations, which are most efficiently performed if a potential energy surface (PES) is available. We therefore present a brief review of the DFs that now exist, also considering their performance on databases for $E_{\mathrm{b}}$ for gas phase reactions and DC on metals, and for adsorption to metals. We also consider expressions for SRP-DFs and briefly discuss other electronic structure methods that have addressed the interaction of molecules with metal surfaces. An overview is presented of dynamical models, which make a distinction as to whether or not, and which dissipative channels are modeled, the dissipative channels being surface phonons and electronically non-adiabatic channels such as electron-hole pair excitation. We also discuss the dynamical methods that have been used, such as the quasi-classical trajectory method and quantum dynamical methods like the timedependent wave packet method and the reaction path Hamiltonian method. Limits on the accuracy of these methods are discussed for DC of diatomic and polyatomic molecules on metal surfaces, paying particular attention to reduced dimensionality approximations that still have to be invoked in wave packet calculations on polyatomic molecules like $\mathrm{CH}_{4}$. We also address the accuracy of fitting methods, such as recent machine learning methods (like neural network methods) and the corrugation reducing procedure. In discussing the calculation of observables we emphasize the importance of modeling the properties of the supersonic beams in simulating the sticking probability curves measured in the associated experiments. We show that chemically accurate barrier heights have now been extracted for DC in 11 molecule-metal surface systems, some of which form the most accurate core of the only existing database of $E_{\mathrm{b}}$ for DC reactions on metal surfaces (SBH10). The SRP-DFs (or candidate SRP-DFs) that have been derived show transferability in many cases, i.e., they have been shown also to yield chemically accurate $E_{\mathrm{b}}$ for chemically related systems. This can in principle be exploited in simulating rates of catalyzed reactions on nano-particles containing facets and edges, as SRP-DFs may be transferable among systems in which a molecule dissociates on low index and stepped surfaces of the same metal. In many instances SRP-DFs have allowed important conclusions regarding the mechanisms underlying observed experimental trends. An important recent observation is that SRP-DFT based on semi-local exchange DFs has so far only been successful for systems for which the difference of the metal work function and the molecule's electron affinity exceeds $7 \mathrm{eV}$. A main challenge to SRP-DFT is to extend its applicability to the other systems, which involve a range of important $\mathrm{DC}$ reactions of e.g. $\mathrm{O}_{2}, \mathrm{H}_{2} \mathrm{O}, \mathrm{NH}_{3}, \mathrm{CO}_{2}$, and $\mathrm{CH}_{3} \mathrm{OH}$. Recent calculations employing a PES based on a screened hybrid exchange functional suggest that the road to success may be based on using exchange functionals of this category. rsc.li/pccp

Received 6th January 2021 Accepted 24th March 2021

DOI: $10.1039 / \mathrm{d} 1 \mathrm{cp} 00044$ 


\section{Introduction}

The accurate modeling of dissociative chemisorption (DC) reactions on metal surfaces is of large practical importance to the description of heterogeneous catalysis, by which the majority of chemicals are made. ${ }^{1}$ DC reactions often constitute the elementary reactions that control the rate of the catalyzed process. $^{2,3}$ Well known examples of such reactions include the DC of methane in steam reforming, ${ }^{4}$ which is presently the main commercial process for hydrogen production, and of $\mathrm{N}_{2}$ in ammonia production, ${ }^{5,6}$ which helps feed a large part of the world population. With the theoretical methods now existing one can predict trends in transition metal (TM) catalysis, and which materials should be good catalysts for producing specific chemicals. ${ }^{7}$ However, theory is not yet very accurate for computing rates, as illustrated by errors in the computed rate of ammonia production still being as large as 1-2 orders of magnitude. ${ }^{8}$ On the one hand, it is fortunate that in computing rates of catalyzed processes one can focus on accurately modeling the transition states (TSs) and reaction intermediates with a high degree of rate control. ${ }^{9}$ On the other hand, the ability to accurately compute rates depends crucially on the accuracy with which reactions barrier heights, ${ }^{2,3}$ and more generally, the moleculesurface interaction ${ }^{10}$ can be computed. As discussed further below, this poses a major problem to the accurate calculation of rates of heterogeneously catalyzed processes, and the catalysis literature has emphasized the need for higher accuracy than afforded by current density functionals (DFs) for achieving predictive power. ${ }^{11}$

Dissociative chemical reactions are also of interest for fundamental reasons. Initial state selected reaction probabilities may depend strongly on the initial vibrational and rotational state of the molecule, ${ }^{12-14}$ and such findings provide information on the geometry of the TS to the reaction. ${ }^{15,16}$ Experiments can also determine how strongly the reaction probability depends on the initial orientation of the molecule with respect to the surface, and on whether the surface can act to reorient the molecule while it travels to the barrier. ${ }^{17,18}$ Experiments on polyatomic molecules have also shown that different vibrations may exhibit different

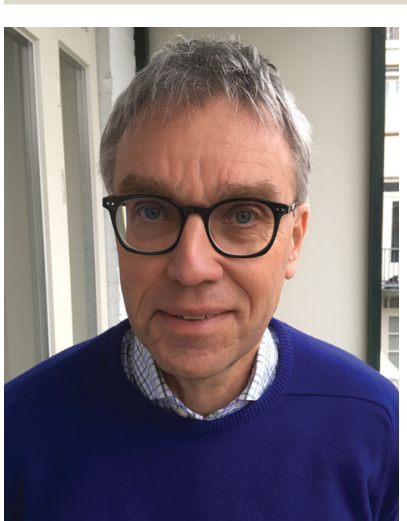

Geert-Jan Kroes
Dr Geert-Jan Kroes obtained his $\mathrm{PhD}$ in Chemistry in 1990 at the University of Amsterdam (The Netherlands), working under the supervision of Prof. R. P. H. Rettschnick. He worked as a postdoc with David Clary from 1990 to 1992 (Cambridge, UK), and with Marc van Hemert and Ewine van Dishoeck from 1992 to 1993 (Leiden, The Netherlands). He worked as a KNAW-fellow at the Free University of Amsterdam and at Leiden University from 1993-1998. At Leiden he became Assistant Professor in 1998, and Full Professor in 2003. His research is focused on reactive scattering of molecules from metal surfaces. efficacies for promoting reaction, ${ }^{19}$ and that the dissociation reaction may be bond selective. ${ }^{20}$ Reactions can be strongly promoted by surface atom motion through increasing the surface temperature $\left(T_{\mathrm{s}}\right),{ }^{21}$ and it has been argued that for specific systems the DC can be affected by electron-hole pair (ehp) excitation. ${ }^{22,23}$ Experiments on vibrationally inelastic scattering ${ }^{24,25}$ and on diffractive scattering ${ }^{26}$ (which has recently become measurable also for polyatomic molecules like methane ${ }^{27}$ ) may also yield information on the geometry of the TS. Modeling these experiments to obtain a thorough understanding of the physics underlying the trends revealed by the experiments requires an accurate description of the molecule-surface interaction. ${ }^{28-30}$

The main challenge to theorists aiming for an accurate description of DC reactions on metals is the present absence of a first principles electronic structure method that can compute molecule-metal surface interaction energies and the corresponding reaction barriers with chemical accuracy (with a generally accepted definition being $1 \mathrm{kcal} \mathrm{mol}^{-131}$ ). With the semi-local DFs that are applicable in developing potential energy surfaces (PESs) with density functional theory (DFT) or in density functional theory molecular dynamics (DFMD) calculations for DC on metals, the best available DF for gas phase reactions (MN-15L, a meta-gradient approximation functional) shows a mean unsigned error of $1.7 \mathrm{kcal} \mathrm{mol}^{-1}$ for barrier heights. ${ }^{32}$ However, this DF, which was obtained semi-empirically by fitting to several databases, ${ }^{32}$ actually shows a mean unsigned error (MUE) of $4.1 \mathrm{kcal} \mathrm{mol}^{-1}$ for molecular adsorption of closed-shell molecules on metals. ${ }^{33}$ More importantly, first principle methods that can at least compute chemically accurate interaction energies for a few points have not yet been demonstrated. Diffusion Monte-Carlo (DMC) calculations on the benchmark $\mathrm{H}_{2}+\mathrm{Cu}(111)$ reaction underestimated the best available value of the reaction barrier height $\left(E_{\mathrm{b}}\right)$ by $1.6 \pm 1.0 \mathrm{kcal} \mathrm{mol}^{-1} \cdot 34$ Dynamics calculations based on an embedded correlated wave function (ECW) method were able to reproduce dissociation probabilities of $\mathrm{O}_{2}$ scattering from $\mathrm{Al}(111)$ with high accuracy, but not yet with chemical accuracy. ${ }^{35}$ While for gas phase reactions of small molecules highly accurate $\operatorname{CCSD}(T)^{36} E_{\mathrm{b}}$ are available in several databases, ${ }^{37,38}$ databases for $E_{\mathrm{b}}$ based on accurate first principles method are simply absent for DC on metals.

In the absence of chemically accurate first principles methods for molecules interacting with metals, the fact that a reaction barrier is not a direct observable poses an extra challenge. Specifically, a reaction barrier can obviously be computed with chemical accuracy using a TS search with a chemically accurate electronic structure method, but in the absence of such an accurate method this approach is not useful. An alternative approach uses a dynamics method to compute a physical quantity that is an observable (the reaction probability curve, i.e., the initial sticking coefficient $\left(S_{0}\right)$ as a function of the incidence energy $\left(E_{i}\right)$, which can be measured in a supersonic molecular beam (MB) experiment). Such an experiment is able to probe the reactivity of well-defined, specific Miller index surfaces, making it suitable for validation. In contrast, in kinetics experiments performed under thermal conditions the reaction often takes place at steps, kinks, vacancies or other defects, ${ }^{39,40}$ 
making these experiments unsuitable for validation purposes, as the computational determination of the barrier geometry becomes ambiguous due to the surface geometry of the reaction site(s) not being well defined. ${ }^{41}$

The solution to the problem posed by the two challenges discussed above, which we will discuss in this review, is based on the specific reaction parameter (SRP) approach to density functional theory (SRP-DFT), which was first developed by Truhlar and co-workers for reactions taking place in the condensed phase. ${ }^{42}$ In the procedure used for DC on a metal surface, a suitable trial DF is constructed, with usually one adjustable parameter. ${ }^{43}$ Next, dynamics calculations are performed, either on the basis of a $\mathrm{PES}^{43}$ or with direct dynamics, ${ }^{44}$ and the parameter (and possibly the form) of the DF is adjusted until the measured $S_{0}$ is reproduced to within chemical accuracy. Once that has been achieved, and after it has been demonstrated that dynamics calculations on the basis of the determined DF can also reproduce another experiment on the same system with chemical accuracy, the DF is called a SRP density functional (SRP-DF). ${ }^{43,44}$ The procedure is obviously semi-empirical, and as designed it is also specific to the system investigated. An advantage of SRP-DFT is that it allows chemically accurate $E_{\mathrm{b}}$ to be extracted, as can be argued ${ }^{43}$ on the basis of the so-called hole model. ${ }^{45}$ Also, it has already been shown that SRP-DFs may exhibit transferability, i.e., a SRP-DF designed to reproduce results for a specific system may also yield chemically accurate results for chemically related systems. ${ }^{46-48}$ As will be discussed below, the SRP-DFT approach has now resulted in chemically accurate barriers for 11 molecule-surface reactions. The SRP-DFT approach has provided the most accurate core of the only existing database for barriers for DC of small molecules on metal surfaces (SBH10), which takes all its carefully chosen references from experiments. ${ }^{49}$

The need to compare with experiments based on dynamics calculations leads to yet more challenges. While it may be possible to accurately model dissociation of $\mathrm{H}_{2}$ on cold metal surfaces with the static surface approximation, ${ }^{28}$ for accurate results it is usually necessary to model surface atom motion for molecules heavier than $\mathrm{H}_{2}$ and its isotopologues. ${ }^{50-53}$ Furthermore, DC on metals may be affected by ehp excitation, ${ }^{22,54}$ and there is uncertainty about how to best model this effect on reaction. ${ }^{55-59}$ Quantum dynamics (QD) calculations on reaction of polyatomic molecules like $\mathrm{CH}_{4}$ or $\mathrm{H}_{2} \mathrm{O}$ on metal surfaces necessarily involve approximations. ${ }^{50,60,61}$ Direct dynamics ${ }^{44,47}$ or quasi-classical trajectory (QCT) calculations ${ }^{62,63}$ can model motion in all molecular degrees of freedom (DOFs) and in the surface atom motion. However, the application of these methods requires careful thought about the experimental conditions for which they should produce valid results. ${ }^{44,47}$ Furthermore, because the calculation of reaction probabilities $<0.01$ is not yet within reach of direct dynamics methods, such calculations require the presence of a PES also describing the dependence of the molecule-surface interaction on the surface atom vibrations (surface phonons). ${ }^{53,62,63}$

In the present state-of-the art in reaction dynamics, moleculemetal surface reactions are still often modeled with standard DFs at the generalized gradient approximation (GGA) level, like
PBE, ${ }^{21,51,53,58,62,64-97}$ its predecessor PW91, ${ }^{53,61,65,78,86,98-129}$ and RPBE. ${ }^{59,65,74,78,85,86,93,101,110,116,117,124,130-142}$ However, GGAexchange DFs combined with correlation DFs approximately describing the attractive van der Waals interaction are increasingly used. $^{101,143-151}$ Researchers are also starting to use meta-GGA (mGGA) DFs. ${ }^{151,152}$ Goals of these calculations include validating models, ${ }^{61,62,65,73,74,76,86,89,98,100,102,105,106,123,145,153}$ analyzing features of the reaction mechanism, $21,51,53,58,64-66,69-72,75,77,79-82,87,90-97,99$, 103,104,108,109,111,114,118,119,121,122,124,126,128,129,131,135-137,140-143 making recommendations for improved catalysts ${ }^{67,68,83,107,130}$ or for better controlling the outcome of a reaction, ${ }^{144}$ and improving the description of experiments. ${ }^{59,62,78,85,88,101,110,112,113,115-117,125}$, 127,132,134,138,139,146-151,154 However, increasingly studies are performed with the aim of developing an SRP-DF, 43,44,46,47,152,155-159 or in which an SRP-DF is used that was specifically designed for the system under study, ${ }^{57,84,102,150,153,160-177}$ or an SRP-DF for a closely related system is used. ${ }^{48,63,166,178-192}$ Also, increasingly experiments are performed with the aim of testing the predictions of calculations using SRP-DFs. ${ }^{23,193,194}$ Recently, the first study we are aware of has been published ${ }^{35}$ that used a PES developed on the basis of ECW calculations, i.e., for $\mathrm{O}_{2}+\mathrm{Al}(111)$. While many studies are still performed with the Born-Oppenheimer (BO) static surface

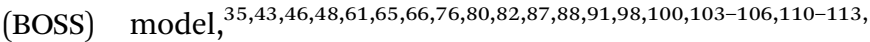
115-117,119-121,123,132,134,137,143,144,148,150-152,156,158,159,163,169,176,177,186,187

increasingly studies explicitly take into account surface atom motion $^{44,47,51,63,70,83-86,89,92-94,96,101,107,118,124,136,139,141,153,157,164-166 \text {, }}$ 170-173,175,181-185,188-191 or ehp excitation, ${ }^{57,58,64,85,95,97,99,122,124,129,140,174}$ or both. ${ }^{59,69,74,109,114,138,142,143,146,147,168,180,192}$ In some cases, ehp excitation as well as non-adiabatic electron transfer was modeled. ${ }^{125-128}$

To give an idea of the kind of accuracy that can be achieved with standard semi-local DFs, we consider three examples that compare theory with experiment. $S_{0}$ computed $^{151}$ for $\mathrm{H}_{2}+\mathrm{Ru}(0001)$ on the basis of the PBE, ${ }^{195} \mathrm{RPBE},{ }^{196} \mathrm{WC},{ }^{197} \mathrm{HTBS}^{198}$ and revTPSS ${ }^{199}$ DFs are compared with experiment ${ }^{200}$ in Fig. 1. As often seen, PBE

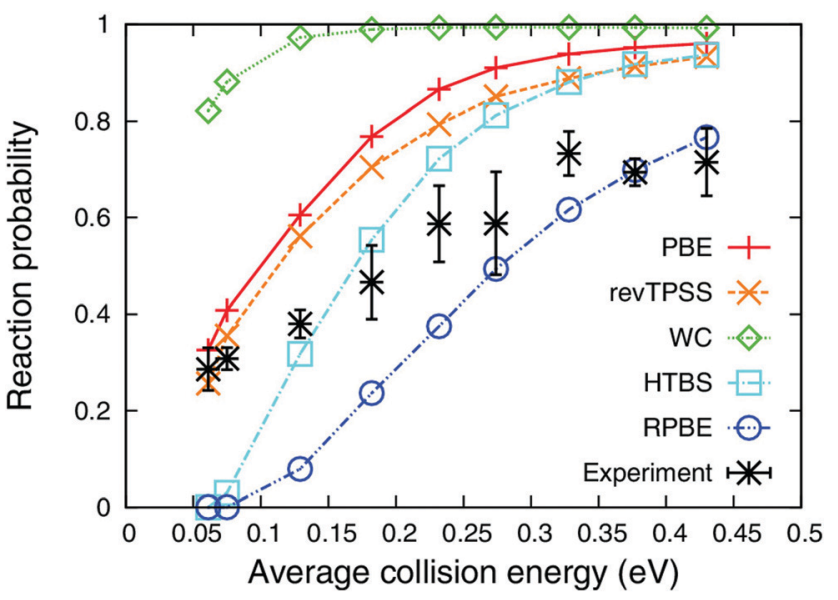

Fig. 1 Experimental $S_{0}$ are compared with computed $\mathrm{S}_{0}{ }^{151}$ for $\mathrm{MBs}$ of $\mathrm{H}_{2}$ dissociating on Ru(0001), for the PBE, ${ }^{195} \mathrm{WC}^{197}{ }^{197} \mathrm{RPE}^{196} \mathrm{HTBS}^{198}$ and revTPSS ${ }^{199}$ DFs. Reprinted from [M. Wijzenbroek and G. J. Kroes, The effect of the exchange-correlation functional on $\mathrm{H}_{2}$ dissociation on $\mathrm{Ru}(0001)$, J. Chem. Phys. 2014, 140, 084702], with the permission of AIP Publishing. 
leads to overestimated reaction probabilities, and RPBE to underestimated $S_{0}$. The WC DF developed for solid state applications overestimates reaction even more than PBE, and the HTBS DF yields a $S_{0}$ curve with a slope that is too high. The mGGA revTPSS DF improves over PBE but still largely overestimates the measured $S_{0}$. As the dynamics model used is basically not in doubt, PESs were accurately fitted, and the properties of the MBs were considered in the calculations, the comparison in Fig. 1 nicely illustrates the difficulties one may encounter when attempting to model the sticking of a molecule on a metal surface using standard semi-local DFs.

$S_{0}$ computed $^{73}$ for $\mathrm{CH}_{4}+\mathrm{Ni}(100)$ on the basis of the PBE $\mathrm{DF}^{195}$ are compared to results from experiments ${ }^{201,202}$ in Fig. 2. Here, the agreement between theory and experiment is quite good. However, in the experiments a significant fraction of methane in the beam should be in excited vibrational states at high $E_{i}$, which should enhance the $S_{0}$, but excited vibrational states were not considered in the theory in Fig. 2. Furthermore, the QD calculations only considered 8 of the 15 DOFs of methane (see also Section 4). It is therefore quite possible that the good agreement found results from cancellation of errors. DFMD calculations on sticking of $\mathrm{CHD}_{3}$ on $\mathrm{Pt}(111)$ found that the use of the PBE DF leads to a significant overestimation of the measured $S_{0}{ }^{51}$ While the type of comparison made in Fig. 2 may be quite informative, it does not yet allow rigorous conclusions to be drawn on the quality of the DF used, due to uncertainties in the validity of the dynamical model for making quantitative predictions.

Finally, $S_{0}$ computed ${ }^{71}$ for DC of $\mathrm{CO}_{2}$ on $\mathrm{Ni}(100)$ on the basis of the PBE $\mathrm{DF}^{195}$ with the reaction path Hamiltonian (RPH) method, ${ }^{50,203}$ and with the quasi-classical trajectory (QCT) method, ${ }^{92}$ are compared to experimental results ${ }^{204}$ in Fig. 3. The problem addressed is very challenging, as the PES is characterized by a reaction path with two TSs, with a chemisorbed precursor

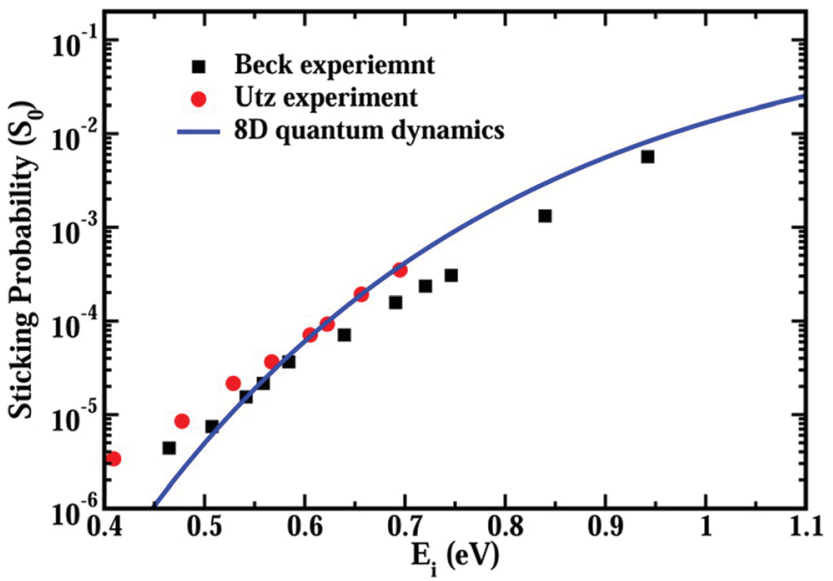

Fig. $2 S_{0}$ computed $^{73}$ with 8D QD calculations for $\mathrm{CH}_{4}$ initially in its rovibrational ground state dissociating on $\mathrm{Ni}(100)$ (blue line). The theoretical results are compared with results of laser-off experiments performed in the Utz group ${ }^{201}$ (red circles) and in the Beck group ${ }^{202}$ (black squares). Reprinted with permission from (X. J. Shen, Z. J. Zhang and D. H. Zhang, Eight-dimensional quantum dynamics strudy of $\mathrm{CH}_{4}$ and $\mathrm{CD}_{4}$ dissociation on Ni(100) surface, J. Phys. Chem. C, 2016, 120, 20199-20205). Copyright (2016) American Chemical Society.

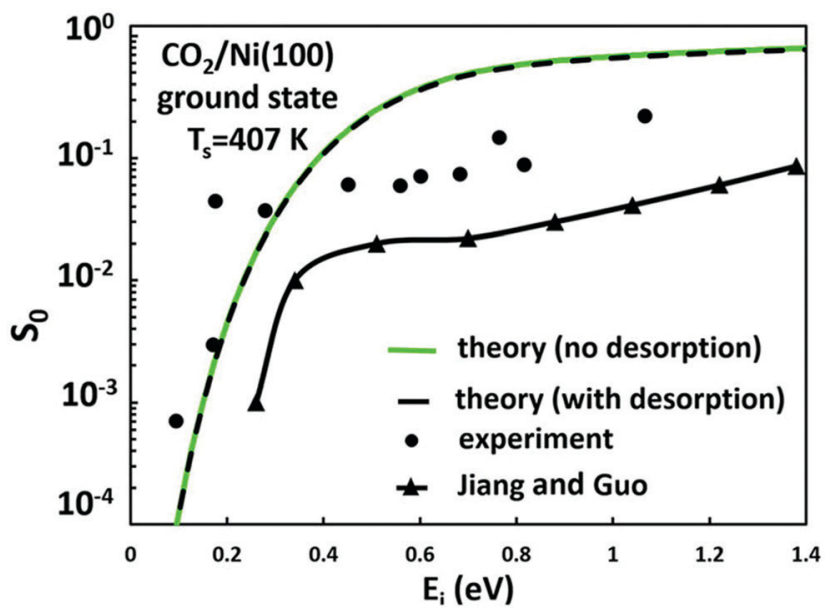

Fig. $3 S_{0}$ computed ${ }^{71}$ with the $\mathrm{RPH}$ method for $\mathrm{CO}_{2}$ dissociating on $\mathrm{Ni}(100)$, with (black line) and without (green line) a correction made for desorption. The black circles show experimental results ${ }^{204}$ and the black triangles results of QCT calculations. ${ }^{92}$ Reprinted from [A. Farjamnia, B. Jackson, The dissociative chemisorption of $\mathrm{CO}_{2}$ on $\mathrm{Ni}(100)$ : a quantum dynamics study, J. Chem. Phys., 2017, 146, 074704], with the permission of AIP Publishing.

molecule in between. ${ }^{71}$ Ref. ref. 71 considered the effect of surface atom motion and modeled all molecular DOFs with QD, albeit with some dynamical approximations. Ref. ref. 92 treated the surface as static and used QCT to likewise model motion in all molecular DOFs. As can be seen, neither theoretical study was able to reproduce the experiment. Using DFMD to additionally model surface phonon motion led to a slight improvement ${ }^{70}$ over the static surface QCT results, ${ }^{92}$ but still the experimental reaction probability was overestimated. Like that in Fig. 2, the type of comparison made in Fig. 3 may be informative, but it does not yet allow rigorous conclusions to be drawn on the quality of the DF used, due to uncertainties in the validity of the dynamical model, and the complexity of the $\mathrm{CO}_{2}$-metal surface PES.

A large range of experiments is available on diatomic molecules scattering from metal surfaces. Experiments that have been performed during the last 5 years on $\mathrm{H}_{2}$ scattering from metal surfaces investigate how elastic scattering depends on the orientational alignment of $\mathrm{H}_{2}$ with respect to the metal surface, ${ }^{169}$ how metal defects like steps ${ }^{166,194,205,206}$ and kinks ${ }^{206}$ affect the reactivity, and the extent to which DC may depend on the incidence plane. ${ }^{193}$ The question has been addressed to what extent associative desorption from $\mathrm{Au}(111)^{23}$ and from copper surfaces ${ }^{194}$ may be affected by ehp excitation, and a new "slow" channel has been identified in associative desorption. ${ }^{194}$ Very recently, experiments have emerged that address the fully initial and final rotational-state resolved state-to-state scattering of ortho$\mathrm{H}_{2}$ from $\mathrm{Cu}(111)$, including the selection of the initial and final magnetic rotational quantum numbers. ${ }^{207}$ This technique was also demonstrated recently for $\mathrm{H}_{2}$ scattering from an insulator surface, i.e., $\mathrm{LiF}(001) .{ }^{208}$ Older work has, amongst other things, addressed the dependence of the reaction on the initial rovibrational state ${ }^{12}$ and reactant orientational alignment with respect to the surface; ${ }^{17}$ an overview is given in a recent review paper. $^{28}$ 
Other experiments on diatomic molecules reacting on or scattering from metals have been performed on e.g. $\mathrm{N}_{2}, \mathrm{O}_{2}, \mathrm{HCl}$, $\mathrm{CO}$, and NO. Recent experiments on $\mathrm{N}_{2}$ have looked at its vibrationally inelastic scattering from $\mathrm{Pt}(111) .{ }^{209}$ Older experiments have studied its $\mathrm{DC}$ on $\mathrm{Ru}(0001)^{210}$ and on tungsten surfaces. ${ }^{211-213}$ Recent experiments on $\mathrm{O}_{2}$ have investigated the effect of orientational alignment ${ }^{214,215}$ and of the molecular spin state $^{215}$ on its DC on metals, and its DC on $\mathrm{Ru} / \mathrm{Cu}(111)$ and $\mathrm{Cu}(111) .{ }^{216}$ Older experiments have addressed its DC on, for instance, $\mathrm{Al}(111)^{217}$ and $\mathrm{Ag}(111) \cdot{ }^{218}$ The $\mathrm{HCl}+\mathrm{Au}(111)$ system has recently been investigated with challenging experiments on $\mathrm{DC}^{219}$ and on vibrationally inelastic scattering, ${ }^{220}$ and recently experiments have also been performed on vibrationally inelastic scattering of $\mathrm{HCl}$ from $\mathrm{Ag}(111){ }^{221}$ A number of exciting recent experiments have been performed on scattering of $\mathrm{CO}$ from metal surfaces, ${ }^{222-225}$ and on oxidation of CO. ${ }^{226}$ The experiments on vibrationally pre-excited CO scattering from $\mathrm{Au}(111)^{224}$ have been analysed in terms of a quantitative energy landscape involving both a physisorption and a chemisorption state, and a barrier in between. ${ }^{227}$ This analysis was in large part inspired by preceding, but very recent theoretical work demonstrating a long vibrational lifetime of physisorbed $\mathrm{CO}$ on $\mathrm{Au}(111)^{228}$ and fast vibrational relaxation of $\mathrm{CO}$ in the chemisorption well. ${ }^{229}$ Vibrationally inelastic scattering of NO from metal surfaces has become a benchmark system for electronically non-adiabatic scattering from metal surfaces, ${ }^{230,231}$ which continues to receive attention. ${ }^{232-234}$

A host of experiments also exists on the reactive and inelastic scattering of polyatomic molecules from metal surfaces. The DC of methane and its isotopologues has become a paradigm for non-statistical reactive scattering. ${ }^{14,18-20,201}$ New experiments challenging theory continue to evolve on $\mathrm{DC}^{21,44,47,162,165,181,182,235-239}$ as well as vibrationally inelastic ${ }^{240}$ and diffractive ${ }^{27}$ scattering, and physisorption. ${ }^{241}$ Recently initial-state selected DC of water $\left(\mathrm{D}_{2} \mathrm{O}\right)$ was studied for the first time in a supersonic $\mathrm{MB}$ experiment. ${ }^{78}$ Experiments have also addressed reactions on stepped ${ }^{165,182,236,242}$ and kinked ${ }^{181}$ surfaces. Experiments have even determined sticking probabilities for dissociative chemisorption at specific surface sites, making distinctions between terrace and step sites, ${ }^{162,242,243}$ or terrace and kink sites, ${ }^{243}$ sometimes with support from theory helping to identify the reacted species. ${ }^{244}$ Of these experiments the most recent ones also employed initial quantum state selection. ${ }^{162,243}$ Older $\mathrm{MB}$ experiments exist on e.g. the $\mathrm{DC}$ of $\mathrm{NH}_{3}{ }^{245} \mathrm{CH}_{3} \mathrm{OH},{ }^{246} \mathrm{CO}_{2},{ }^{204}$ and ethane ${ }^{247-251}$ and higher alkanes. $^{247,252}$ Recently quantum-state and velocity resolved experiments have been performed on vibrational excitation of acetylene scattering from $\mathrm{Au}(111),{ }^{253}$ and experiments have also addressed trapping-desorption and direct scattering of formaldehyde from $\mathrm{Au}(111)$. $^{254,255}$

This review focuses on applications of SRP-DFT to reactions of small molecules with metal surfaces at the gas solid interface, with emphasis on DC reactions. We will also review electronic structure methods and dynamical models and methods that are applicable to these reactions, and methods for potential surface fitting. However, in reviewing these methods and models we will often refer to references for details. There are several excellent recent reviews and perspective papers that are related to the broader field of scattering of molecules from metal surfaces at the gas solid interface. Topics addressed include DFT for surface chemistry and catalysis, ${ }^{11}$ kinetics of chemical reactions at gas-surface interfaces ${ }^{41}$ and in heterogeneous catalysis, with emphasis on a new powerful pump-probe technique, ${ }^{256}$ surface catalysis modeling, ${ }^{257}$ reactive and non-reactive scattering of $\mathrm{H}_{2}$ from metal surfaces, ${ }^{28} \mathrm{O}_{2}$ activation by metal surfaces, ${ }^{258}$ dissociation dynamics of methane on TM surfaces, ${ }^{259}$ energetics and dynamics of methane and water dissociation on transition metal surfaces, ${ }^{260}$ quantum state-resolved studies of chemisorption reactions, ${ }^{261,262}$ electronically non-adiabatic effects in surface chemistry and dynamics ${ }^{263-266}$ and how to address these effects, ${ }^{255,267}$ effects of surface atom motion on dissociation reactions, ${ }^{30,266}$ mode-specificity and bond-selectivity in QD descriptions of reactions of polyatomic molecules on metals, ${ }^{29}$ and how experiments can test central assumptions made in theory applied to the dynamics of molecular interactions and chemical reactions at metal surfaces. ${ }^{268} \mathrm{~A}$ recent theoretical perspective on the dynamics of reactions on metal surfaces was published by Jiang and Guo. ${ }^{269}$

As noted this review focuses on dissociative chemisorption of molecules on metal surfaces at the gas-solid interface. Of course research is taking place in closely related areas, i.e., reactions of molecules at the gas-liquid interface, reactions at the surface of metal clusters or nano particles, and gas phase reactions. There is a growing body of work on interactions of molecules with metal surfaces at the solid-liquid interface, with important applications being electrochemistry and heterogeneous catalysis, and potentially incorporating research on grand challenges. ${ }^{270}$ Review papers emphasizing theory that can serve as an introduction to this area concern the structure of water at metal interfaces, ${ }^{271}$ the modeling of electrochemical interfaces, ${ }^{272-274}$ and heterogeneous catalysis. ${ }^{275}$ Some trends in this area are to perform research on implicit solvent models, ${ }^{276-279}$ to investigate the use of neural networks to model the metal-liquid interface, ${ }^{280}$ and to investigate whether scaling relationships found for the gas-solid interface also hold for the gas-liquid interface. ${ }^{281}$ Reviews on reactions at interfaces (whether gaseous or solid) with metal clusters include ref. 282-284; also in this area attention is being paid to ${ }^{282}$ developing affordable solvent models. Finally, there is a lot of cross-fertilization between theoretical molecule-surface reaction dynamics and gas phase reaction dynamics, e.g., concerning how potential energy surfaces should be fitted, how dynamics calculations should be performed, and how reaction mechanisms can be interpreted. The review paper by Zhang and Guo ${ }^{285}$ and the perspective paper of Guo and co-workers ${ }^{286}$ can serve as useful introductions to this research area.

The outline of this review is as follows. Section 2 gives an overview of electronic structure methods applicable to molecules interacting with metal surfaces. Section 2.1 gives a brief description of standard DFT, including the performance of standard DFs on databases of reaction barriers and adsorption energies to metals. In Section 2.2 we describe the SRP approach to DFT. Section 2.3 very briefly discusses tight binding methods that are 
closely related to DFT, and Section 2.4 briefly describes methods other than DFT that have been applied to molecules interacting with metal surfaces. Section 3 discusses the fitting of PESs to electronic structure results, which is necessary if a direct dynamics method is not used. Section 3.1 discusses the use of neural network potentials (NNPs), Section 3.2 briefly touches on the corrugation reducing procedure (CRP), and Section 3.3 briefly discusses other fitting methods. Section 4 gives a brief overview of dynamical models and methods that can be employed to model reactive and non-reactive scattering of molecules from metal surfaces. Section 4.1 describes dynamical models, which is related to the approximations that are made in scattering calculations. Section 4.2 discusses the classical trajectory (CT) methods that are being used in dynamics calculations. In Section 4.3 we briefly describe on the fly dynamics methods, like DFMD. Section 4.4 describes QD methods, including their performance on DC of $\mathrm{H}_{2} \mathrm{O}$ and $\mathrm{CH}_{4}$, and approximations often invoked to deal with motion along the surface, rotational motion, vibrational motion, and surface atom motion. Section 4.5 gives a summary description of the ring polymer molecular dynamics (RPMD) method. Section 4.6 discusses the calculation of observables, and how the usefulness of comparing computed $S_{0}$ to experimental values depends on the availability of accurate information regarding the molecular beams employed.

In Section 5 we turn to the results that have been obtained with SRP-DFs. In Section 5.1 we describe the systems for which SRP-DFs and in Section 5.2 the systems for which candidate SRP-DFs have been derived. In Section 5.3 we describe systems for which attempts to derive SRP-DFs have so far failed and in Section 5.4 we present calculations using SRP-DFs for related systems. Section 6 provides additional discussion. Topics considered are for which systems the SRP-DFT approach based on GGA exchange DFs works, and why (Section 6.1), strategies for deriving SRP-DFs, and why SRP-DFT works (Section 6.2). We also consider to what extent SRP-DFs are transferable among chemically similar systems and the potential use of transferability in modeling heterogeneous catalysis (Section 6.3), the performance of general purpose DFs (Section 6.4), challenges facing SRP-DFT (Section 6.5), the importance of using a correct dynamical model (Section 6.6), how dynamics based on SRPDFs helps with interpreting dynamical effects (Section 6.7), and predictions made with SRP-DFs that are in need of experimental testing (Section 6.8). Section 7 presents conclusions.

\section{Electronic structure theory}

Dynamics calculations, which are needed to compute the reaction probabilities for comparison with MB sticking experiments, depend on electronic structure theory for either the PES or the forces (the latter are obtained without the need for a PES in direct dynamics calculations). Electronic structure methods we will discuss in this section include DFT, SRP-DFT, and other methods.

\subsection{DFT}

Most dynamics calculations on reactive scattering of molecules from metal surfaces use a PES or forces taken from DFT. ${ }^{287,288}$
Most of these studies have relied on DFs at the GGA level of theory, in which the DF only depends on the electron density and its gradient, while some studies have used DFs at the mGGA level, in which the DF also depends on the second derivative of the density, or on the kinetic energy density. ${ }^{289,290}$ Below we will discuss some much used DFs, with reference to the accuracy achievable with these DFs for $E_{\mathrm{b}}$ for gas phase reactions, for adsorption energies of molecules to TM surfaces, and for $E_{\mathrm{b}}$ for DC of molecules on metal surfaces. Much of what will be stated below on DFT is based on recent general reviews and perspectives on DFT, ${ }^{290-294}$ recent reviews on DFT for van der Waals energies and forces, ${ }^{295-297}$ and a recent review on random phase approximation methods ${ }^{298}$ (which may be viewed as rung $5 \mathrm{DFs},{ }^{299}$ see below).

DFs may be classified in several ways, for instance, according to the "rung on Jacob's ladder" they occupy ${ }^{299}$ (see Fig. 4), according to whether they are based on non-empirical constraints or on semi-empirical parameterization, ${ }^{293}$ or according to whether they were developed to work on specific problems, or as general purpose ("universal") DF. ${ }^{293}$ We will discuss them by "rung" first, also noting that the rung determines whether a DF is "semi-local" or "non-local". Specifically, DFs on the first three rungs (LDA, GGA, and mGGA) are semi-local. This means that they can be evaluated efficiently through a three-dimensional (3D, in this work " $n$-dimensional” will be abbreviated as “ $n \mathrm{D}$ ”) integral over space, evaluating the action of the DF on the density, and possibly its gradient, and possibly the kinetic energy density at each point in space. $^{299}$ The DFs on rungs 4 and 5 are much harder to evaluate,

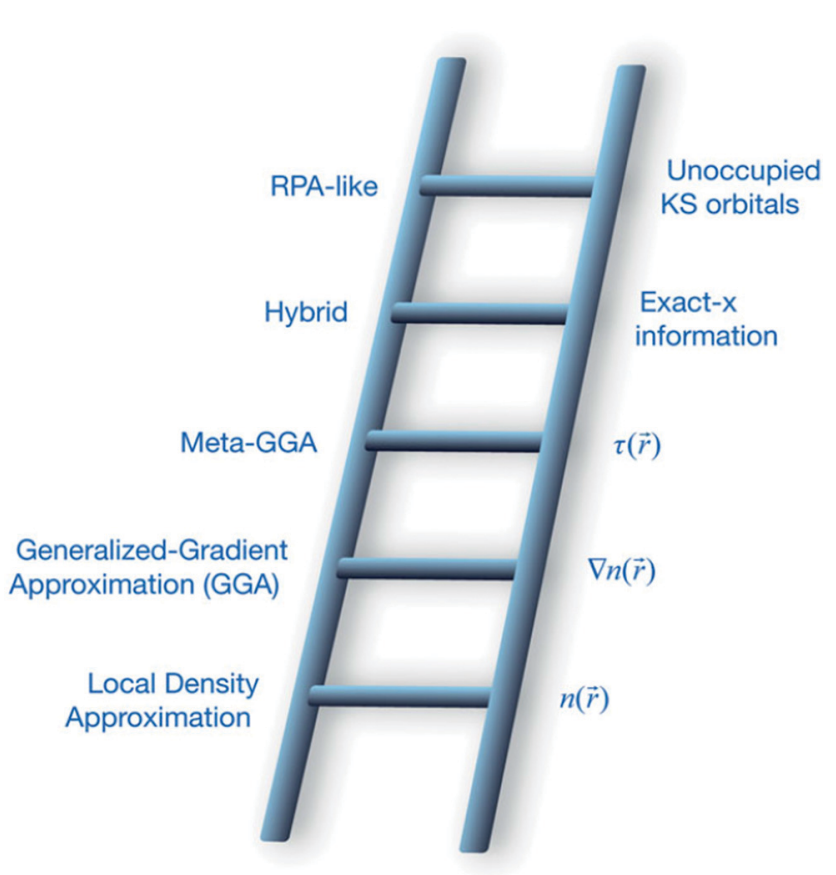

Fig. 4 Jacob's ladder of DF approximations. ${ }^{299}$ The rung names are shown on the left, and their "ingredients" on the right. Reprinted by permission from Springer Nature Customer Service GmbH: Springer Nature, MRS Bull., Climbing the ladder of density functional approximations, J. P. Perdew, vol. 38, pp. 743-750, Copyright 2013. 
and take much more computer time. ${ }^{299}$ Within each rung we will distinguish between non-empirical and semi-empirical DFs, and in some cases discuss whether they were developed to be general purpose DFs, or for a specific class of problems. In classifying the DFs by their rung some of the functionals using correlation DFs for an approximate description of the attractive van der Waals interaction which, strictly speaking, are non-local although they can usually be efficiently evaluated, will be classified as semi-local.

2.1.1. Benchmarking DFT: databases. The accuracy of DFs can be benchmarked by testing them on databases of properties of for instance molecules, TM surfaces, and molecules interacting with TM surfaces. Databases that are important in this context concern $E_{\mathrm{b}}$ for gas phase reactions and for reactions of molecules with TMs, and of adsorption energies of atoms and molecules to TM surfaces. Databases that will be mentioned below include the DBH24/08 database (24 diverse barrier heights for gas phase reactions), ${ }^{37}$ the $\mathrm{B} 76$ database (38 barrier heights for hydrogen-atom transfer (HTBH38/08), and 38 barrier heights for non-hydrogen atom transfer reactions (NHTB38/08), see ref. 289 and references therein), and the $\mathrm{BH} 206$ database (206 barrier heights for gas phase reactions). ${ }^{31}$ The $\mathrm{DBH} 24 / 08 E_{\mathrm{b}}$ are either derived from accurate experiments, or they come from high level theory (the Weizman-1 or the multi-reference configuration interaction (MRCI) method ${ }^{300}$ ).

SBH10 is a recently developed database containing 10 barrier heights for DC reactions on TM surfaces. ${ }^{301}$ The ADS41 database $^{302}$ contains 26 entries for chemisorption energies (dissociative as well as molecular) on TMs (CE26) and 15 entries for adsorption energies dominated by van der Waals interactions. This is basically an extension with one chemisorption system and one physisorption system of the CE39 database of Wellendorff et al., ${ }^{303}$ which contained 25 entries for chemisorption energies on TMs $\left(\mathrm{CE} 25^{303}\right)$ and 14 physisorption energies. In turn, the CE27 database d $^{304,305}$ is a predecessor of the CE25

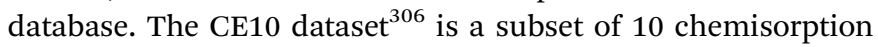
energies chosen from the CE25 database. A variant of CE39 (which we call CE39b) exists in which the gas phase dissociation of dissociatively adsorbing molecules is factored out, so that only molecular and atomic chemisorption and physisorption are considered. ${ }^{33}$ Similarly, the CE21b database is a subset of the CE25 set with the gas phase dissociation of dissociatively adsorbing molecules factored out. ${ }^{307}$ The CE39 and ADS41 databases have recently been superseded by the CE81 database, ${ }^{308}$ which contains dissociative adsorption and molecular adsorption energies for 81 systems. This database has, to our knowledge, not yet been used for benchmarking purposes. The estimated accuracy of the CE39 and CE81 databases is about $3.0 \mathrm{~kJ} \mathrm{~mol}^{-1}$ per adsorbed fragment. ${ }^{308}$

A further possibility for benchmarking that we have not yet mentioned, and that we have not used in our review, is to compare with measured vibrational frequencies of molecules adsorbed to metal surfaces. Adsorbate vibrational frequencies can be considered to be a fingerprint of the molecule-surface interaction. ${ }^{309}$ Sophisticated methods like the vibrational selfconsistent field and the vibrational configuration-interaction method, ${ }^{310}$ and collocation methods ${ }^{311}$ can be used to compute accurate vibrational energies of molecules adsorbed to surfaces.
2.1.2. Rung 1: the local density approximation. At the lowest or first rung ${ }^{299}$ the local density approximation (LDA) ${ }^{288}$ or local spin density approximation (LSDA) is found. There are some variants according to how exactly the correlation energy is computed from Quantum Monte-Carlo (QMC) data, ${ }^{31}$ but these distinctions are not important within the present framework. As can be seen from Tables 1 and 2, the LDA is not accurate for $E_{\mathrm{b}}$ of gas phase reactions, nor is it accurate for adsorption energies. The LDA satisfies a number of constraints and is good for some aspects of solid state physics like lattice constants and surface energies, but it is not good for chemistry. ${ }^{299}$

2.1.3. Rung 2: DFs based on GGA exchange. The second rung contains the GGA DFs, which also use the gradient of the electron density. Well-known examples of this class that have been used as a basis for surface reaction dynamics calculations include the PW91 DF, ${ }^{312}$ its successor $\mathrm{PBE},{ }^{195}$ the B88P86 DF, ${ }^{313}$ and the RPBE DF. ${ }^{196}$ The PBEsol $\mathrm{DF}^{314}$ is not usually used for calculations on reaction dynamics or surface adsorption. The PBE,

Table 1 Mean signed errors (MSE), mean unsigned errors (MUE), and root-mean-square errors (RMSE) for gas phase reaction barrier heights computed for the DBH24/08, the combined HTBTH38/08 and NHTBH38/ 08 (called B76 here), and the $\mathrm{DBH} 206$ databases are presented for selected DFs of different types. All values are in $\mathrm{kcal} \mathrm{mol}^{-1}$. DBH24/08 results are mostly from studies by Truhlar and co-workers ${ }^{738}$ and Wellendorff et al., ${ }^{304}$ the B76 results from a study by Peverati and Truhlar, ${ }^{289}$ and the $\mathrm{DBH} 2 \mathrm{O} 6$ results from a study by Mardirossian and Head-Gordon ${ }^{31}$

\begin{tabular}{|c|c|c|c|c|c|c|}
\hline \multirow[b]{2}{*}{ DF } & \multirow{2}{*}{$\frac{\text { Database }}{\text { Type DF }}$} & \multicolumn{3}{|c|}{ DBH24/08 } & \multirow{2}{*}{$\frac{\text { B76 }}{\text { MUE }}$} & \multirow{2}{*}{$\frac{\mathrm{DBH} 206}{\mathrm{RMSE}}$} \\
\hline & & MSE & MUE & RMSE & & \\
\hline LDA & LDA & -13.4 & 13.4 & 16.8 & 15.0 & 19.58 \\
\hline PBEsol & GGA & -10.4 & 10.4 & - & 11.3 & 13.2 \\
\hline PW91 & GGA & -8.5 & 8.5 & - & 9.2 & 9.56 \\
\hline B88P86 & GGA & -8.4 & 8.4 & - & 8.9 & 8.95 \\
\hline PBE & GGA & -8.2 & 8.2 & 9.9 & 8.9 & 9.17 \\
\hline RPBE & GGA & -6.3 & 6.3 & 7.8 & 6.6 & 7.62 \\
\hline N12 & NGA & - & - & - & $6.9^{316}$ & 7.09 \\
\hline MOHLYP & GGA & -5.6 & 5.7 & - & 5.6 & - \\
\hline GAM & NGA & - & - & - & $5.3^{317}$ & 7.22 \\
\hline RPBE-D3 & GGA + LD & - & - & - & - & 8.35 \\
\hline optB88-vdW & $\mathrm{GGA}+\mathrm{NLD}$ & -8.5 & 8.5 & 10.4 & - & - \\
\hline optPBE-vdW & $\mathrm{GGA}+\mathrm{NLD}$ & -7.6 & 7.6 & 9.5 & - & - \\
\hline vdW-DF2 & GGA + NLD & -6.9 & 7.1 & 8.5 & - & - \\
\hline vdW-DF1 & GGA + NLD & -6.2 & 6.5 & 7.8 & - & - \\
\hline BEEF-vdW & $\mathrm{GGA}+\mathrm{NLD}$ & -6.0 & 6.0 & 7.6 & - & - \\
\hline revTPSS & mGGA & -8.1 & 8.1 & 9.5 & 8.0 & 7.44 \\
\hline SCAN & mGGA & - & - & - & - & 7.57 \\
\hline M06-L & mGGA & -3.2 & 4.1 & - & 4.0 & 6.9 \\
\hline MS2 & mGGA & - & - & - & $6.2^{739}$ & 6.20 \\
\hline MBEEF & mGGA & - & - & - & - & 5.43 \\
\hline MN12-L & mNGA & - & - & - & 1.8 & 4.29 \\
\hline MN15-L & mNGA & - & - & - & $1.7^{32}$ & 4.78 \\
\hline B97M-V & mGGA + NLD & - & - & - & - & 4.35 \\
\hline B3LYP & H GGA & -4.1 & 4.2 & - & 4.4 & 5.96 \\
\hline HSE06 & SH GGA & - & - & - & 4.0 & - \\
\hline M08-HX & H mGGA & 0.2 & 1.1 & - & 0.97 & 1.80 \\
\hline MN12-SX & SH mGGA & - & - & - & 1.15 & 3.05 \\
\hline$\omega \mathrm{B} 97 \mathrm{M}-\mathrm{V}$ & $\begin{array}{l}\text { LCH mGGA + } \\
\text { NLD }\end{array}$ & - & - & - & - & 1.68 \\
\hline RPA & rung 5 & & & & $2.3^{364}$ & \\
\hline $\mathrm{HF}$ & WFT & 8.4 & 8.7 & - & 16.1 & 16.6 \\
\hline MP2 & WFT & 4.7 & 5.0 & - & 4.9 & - \\
\hline QMC(PBE) & Stochastic & & & & $1.2^{423,424}$ & \\
\hline $\operatorname{CCSD}(\mathrm{T})$ & WFT & - & $0.46^{37}$ & - & - & - \\
\hline
\end{tabular}


Table 2 Mean signed errors (MSE), mean unsigned errors (MUE), and root-mean-square errors (RMSE) for adsorption energies of atoms and molecules to metal surfaces, computed for the databases CE10, CE21b, CE39b, CE26 and ADS41 are presented for selected DFs of different types. All values are in $\mathrm{kcal} \mathrm{mol}^{-1}$. CE10 results are taken from ref. 306, CE21b results from ref. 307, CE39b results from ref. 368, and the CE26 and the ADS41 results from ref. 302. Boldfaced entries under the ADS41 and the CE26 headings refer to the very similar CE25 303 and CE39 ${ }^{303}$ datasets, respectively

\begin{tabular}{|c|c|c|c|c|c|c|}
\hline & Database & CE10 & CE21b & CE26 & ADS41 & CE39b \\
\hline LSDA & LDA & - & - & $21.8 /-19.4^{c}$ & - & 19.3 \\
\hline PW91 & GGA & - & - & $7.7 /-4.8^{c}$ & $7.6^{a}$ & 9.3 \\
\hline PBE & GGA & 10.1 & $6.9 /-6.5$ & $7.1 /-4.4 /-2.7^{c}$ & $7.8 / 7.2^{a}$ & 9.6 \\
\hline RPBE & GGA & 5.3 & $3.5 / 1.4$ & $5.3 / 2.1 / 1.0^{a}$ & $8.8 / 7.6^{a}$ & 8.7 \\
\hline GAM & GGA & - & - & - & - & 8.3 \\
\hline optB88-vdW & GGA + NLD & - & - & $9.3 /-6.7^{a}$ & $6.0^{a}$ & - \\
\hline vdW-DF2 & GGA + NLD & & & $6.7 / 3.5$ & & \\
\hline vdW-DF1 & GGA + NLD & & & $4.8 / 2.1$ & & \\
\hline BEEF-vdW & GGA + NLD & 4.4 & - & $4.8 / 0.0 / 0.3^{a}$ & $5.3 / 4.9^{a}$ & 5.8 \\
\hline SW-R88 & sGGA + NLD & - & - & $5.1 / 0.3^{a}$ & $3.4^{a}$ & - \\
\hline SCAN/rVV10 & mGGA + NLD & - & - & $12.0 /-10.6^{d}$ & 8.5 & - \\
\hline MS2 & mGGA & - & - & $6.2 /-3.5$ & 5.8 & - \\
\hline RTPSS & mGGA & - & $4.2 / 0.7$ & - & - & - \\
\hline HSE06 & SH GGA & - & - & $9.2 /-5.3$ & 8.3 & - \\
\hline RPA & rung 5 & 4.8 & - & - & - & - \\
\hline
\end{tabular}

RPBE, and PBEsol DFs are examples of non-empirical, constraintbased DFs. Of these, the PBE DF is usually considered a general purpose DF. The RPBE DF was designed to work well for chemisorption of molecules and atoms to metal surfaces, ${ }^{196}$ and the PBEsol DF for the description of the solid state. ${ }^{314}$ In the words of Perdew, there is an incompatibility within the GGA for constraints: a subset of constraints can be obeyed by a GGA DF, but not all of them at once by the same GGA DF. ${ }^{299}$ This yields some freedom in which constraints to apply: constraint-based DFs may still be designed with a specific class of problems in mind, even though they are usually called "non-empirical". 314 Examples of semiempirical gradient approximation (GA) DFs include MOHLYP, ${ }^{315}$ N12, ${ }^{316}$ and GAM. ${ }^{317}$ The latter two were called non-separable gradient approximation (NGA) DFs by Truhlar and co-workers, by which they meant that these second rung DFs could not be written as a combination of a distinct exchange DF and a distinct correlation DF. ${ }^{316}$ MOHLYP was designed to be good for inorganometallic and organometallic chemistry, N12 to be good for both structural and energetic properties, i.e., general purpose, and GAM to be especially good for homogeneous catalysis involving TMs.

The non-empirical GGA DFs PBE, RPBE, PBEsol, PW91 and B88P86 all systematically underestimate $E_{\mathrm{b}}$ for gas phase reactions, to the point that not only are the mean signed errors (MSEs) all negative, but the mean unsigned errors equal the absolute values of the MSEs for the DB24/08 database (see Table 1). The poor performance of these and similar GGA DFs for gas phase reaction $E_{\mathrm{b}}$ has been used to argue that they should be poor for surface chemistry in general. ${ }^{318,319}$ However, as can be seen in Table 2 some of these GGA DFs perform reasonably well for surface adsorption energies, and results of Garza et $a .^{307}$ and of Wellendorff et $a{ }^{303}$ show that the PBE and RPBE DFs do not systematically underestimate chemisorption energies on TMs (see also Table 2 for the CE21b database). In fact, it is now well known that the RPBE DF often overestimates $E_{\mathrm{b}}$ for DC on TMs, while PW91 and PBE often underestimate $E_{\mathrm{b}} \cdot{ }^{43,116}$ These points are important to SRP-DFT and we will discuss them further below. We note that PBEsol does systematically underestimate chemisorption energies, but this DF was designed for solid state properties like lattice constants and surface energies. It is now well known that GGAs that do well on these properties tend to perform poorly on adsorption energies and gas phase $E_{\mathrm{b}}{ }^{314,320}$ (see also Fig. 5). Note finally that the MOHLYP, N12, and GAM DFs of Truhlar and co-workers perform comparatively well on $E_{\mathrm{b}}$ for gas phase reactions (see Table 1), with the GAM DF exhibiting a MUE of $5.3 \mathrm{kcal} \mathrm{mol}^{-1}$ for the B76 database of $E_{\mathrm{b}}$. GAM also performs comparatively well for adsorption energies (see Table 2), suggesting that this DF might also perform rather well in surface reaction dynamics calculations.

GGA DFs are not capable of describing the van der Waals dispersion interaction. The van der Waals interaction may be added in a cost-effective manner in several ways. One of the simplest methods is by adding pair potentials on the basis of dispersion coefficients, which are dependent on the chemical environment of the atoms in Grimme's DFT-D3 method ${ }^{321}$ (in Tables 1-3 we call this "local dispersion" with acronym LD, just to emphasize that the DF used can still be semi-local). Another version of the DFT-D3 $\operatorname{method}^{322}$ uses a different 


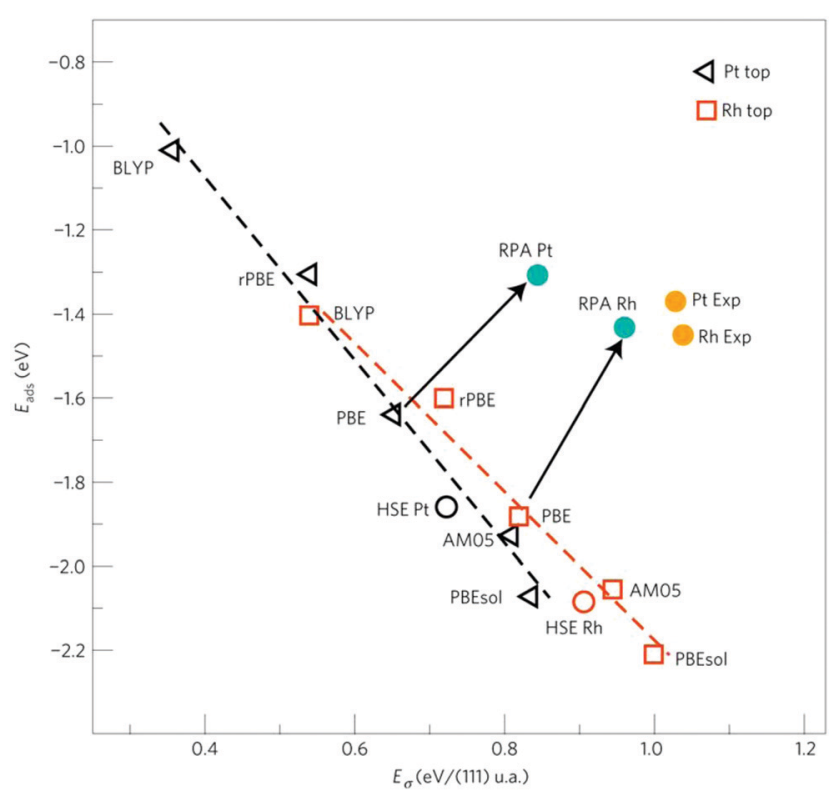

Fig. 5 The adsorption energy at the top site is plotted vs. the surface energy per unit area for $\mathrm{CO}$ on $\mathrm{Pt}(111)$ (black triangles) and $\mathrm{Rh}(111)$ (red squares). ${ }^{320}$ Semi-local functionals used were the AM05, ${ }^{726}$ PBEsol, ${ }^{314}$ $\mathrm{PBE}^{195} \mathrm{RPBE}^{196}$ and BLYP ${ }^{313,353}$ DFs. Results are also shown for the hybrid HSE $^{356}$ DF. Results obtained with the RPA ${ }^{727}$ are shown as blue circles, and experimental results (from ref. 320 and ref. 728 ) as yellow circles. Reprinted by permission from Springer Nature Customer Service GmbH: Nature, Nature Mater., Accurate surface and adsorption energies from many-body perturbation theory, L. Schimka, J. Harl, A. Stroppa, A. Grüneis, M. Marsman, F. Mittendorfer, G. Kresse, vol. 9, pp. 741-744, Copyright 2010.

Table 3 Mean signed errors (MSE), mean unsigned errors (MUE), and root-mean-square errors (RMSE) for reaction barriers on transition metal surfaces for three selected DFs of different types, as computed for the database $\mathrm{SBH} 10 .{ }^{301}$ Errors in adsorption energies of atoms and molecules to transition metal surfaces are also presented, as computed for the database CE26 per adsorbed fragment. All values are in $\mathrm{kcal} \mathrm{mol}^{-1}$. SBH10 results were taken from ref. 301 and the CE26 results from ref. 301 and ref. 302

\begin{tabular}{|c|c|c|c|c|c|c|c|}
\hline \multirow[b]{2}{*}{ DF } & \multirow{2}{*}{$\frac{\text { Database }}{\text { Type DF }}$} & \multicolumn{3}{|c|}{ SBH10 } & \multicolumn{3}{|l|}{ CE26 } \\
\hline & & MSE & MUE & RMSE & MSE & MUE & RMSE \\
\hline BEEF-vdW & GGA + NLD & 0.7 & 2.8 & 3.2 & -0.2 & 3.9 & 4.8 \\
\hline MS2 & mGGA & -7.8 & 8.3 & 9.7 & -3.5 & 5.3 & 6.2 \\
\hline HSE06 & rsHGGA & -9.9 & 9.9 & 10.8 & -5.3 & 7.8 & 9.5 \\
\hline
\end{tabular}

damping function than the original DFT-D3 method, i.e., the so-called Becke-Johnson damping function ${ }^{322}$ (DFT-D3(BJ)), and the original version is sometimes called DFT-D3(0) to distinguish it from this newer version. In a newer version of DFT-D (DFT-D4) $)^{323}$ the effect of charges in a molecule are also taken into account. The method of Tkatchenko and Scheffler ${ }^{324,325}$ may be viewed along similar lines as the Grimme DFT-D methods. ${ }^{295}$

Other methods for incorporating van der Waals dispersion interactions use the non-local correlation DFs (non-local dispersion, acronym NLD) of Lundqvist and Langreth and co-workers (vdW-DF1, ${ }^{326}$ here abbreviated as vdW1, or vdW-DF2, ${ }^{327}$ here abbreviated as vdW2, or a very recent follow-up version $(\mathrm{vdW}-\mathrm{DF} 3)^{328}$ ), or non-local correlation DFs of Vydrov and
Van Voorhis (VV10 $0^{329}$ or its slightly revised rVV10 form ${ }^{330}$ ). These DFs are non-local in the sense that they use densitydependent nonlocal correlation. ${ }^{293}$ Only the vdW1 and vdW2 correlation DFs are strictly non-empirical, in the sense that they do not contain adjustable parameters. ${ }^{295}$ The vdW1 correlation DF has been combined with several exchange DFs in a semiempirical spirit, e.g., in the optPBE-vdW1 $1^{331}$ and the optB88$\mathrm{vdW} 1 \mathrm{DFs}^{331}$ to obtain good interaction energies of weakly interacting dimers, and in the optB86b-vdW ${ }^{332} \mathrm{DF}$ to obtain more accurate lattice constants of solids. The vdW1 correlation DF has also been combined with improved exchange DFs based on theoretical arguments, ${ }^{333,334}$ and the same is true for the vdW2 DF. ${ }^{335}$ Calculations with the vdW1 $\mathrm{DF}^{326}$ correctly describe the scaling of the adsorption energy of molecules on metals with molecular size, ${ }^{295}$ whereas the standard PBE ${ }^{195}$ GGA DF fails to do so. ${ }^{295}$ The vdW2 DF has been incorporated in a DF (called BEEF-vdW) semi-empirically fitted to gas phase reaction barriers, adsorption energies on TM surfaces, and other observables. ${ }^{304}$ Calculations with the vdW1 and vdW2 DFs have become computationally tractable thanks to an efficient implementation by Roman-Perez and Soler, ${ }^{336}$ which is also at the basis of the computational efficiency of the rVV10 DF. ${ }^{330}$

Of the DF approaches incorporating van der Waals dispersion, RPBE-D3 improves the description of the chemisorption and physisorption energies combined in the ADS41 database (Table 2). However, adding D3 dispersion to the RPBE DF worsens the description of chemisorption energies (see the CE26 database results in Table 2), and of gas phase reaction $E_{\mathrm{b}}$ (Table 1), suggesting that adding dispersion in this manner to a GGA might not be useful to surface reaction dynamics. Similar findings concerning gas phase reaction barriers were obtained for other cases were D3 dispersion was added to existing GGA exchange-correlation functionals. ${ }^{31}$ It might be tempting to relate this finding to the way D3 dispersion was constructed $^{321}$ and to conclude that adding D3 dispersion to an existing functional might not work for barriers in general. However, this is not necessarily correct. In fact, the fourthbest performing functional ${ }^{31}$ for the DBH206 database simply combined the hybrid Minnesota functional SOGGA11-X with D3(BJ) dispersion, ${ }^{337}$ and performs better on the DBH206 database than SOGGA11-X itself, ${ }^{31}$ while also performing quite well on non-covalent interactions. ${ }^{337}$ The vdW1 and vdW2 DFs outperform the optPBE-vdW and optB88-vdW DFs for gas phase reaction barriers (Table 1 ) and chemisorption energies on TMs (Table 2). The BEEF-vdW DF is best for these properties, and also gives a rather accurate description of barriers for DC on TMs (with an MUE of only $2.8 \mathrm{kcal} \mathrm{mol}^{-1}$, see Table 3). Note that BEEF-vdW has been fitted semi-empirically to chemisorption energies and gas phase reaction barriers, but not to DC barriers. Finally, we note that adsorption energies can be computed very accurately with an approach based on weighted averages of RPBE adsorption energies and optB86b energies (SW-R88 called here), ${ }^{338}$ but this method is a DFT-based method rather than a DFT method.

2.1.4. Rung 3: DFs based on mGGA exchange. The third rung of DFs also contains the kinetic energy density $\tau$ (one could also use the second derivative of the density, but this may 
lead to less stable calculations ${ }^{293}$ ). According to Perdew, with constraint-based mGGAs good accuracy should be achievable for chemistry at equilibrium, i.e., for typical solids and molecules near their equilibrium geometries. ${ }^{299}$ By now well-known examples of constraint-based mGGA DFs include the revTPSS $^{199}$ and the SCAN ${ }^{339}$ DFs, where the latter obeys all 17 known exact constraints that can be obeyed by a mGGA. ${ }^{339}$

An advantage of inclusion of $\tau$ is that it can be used to determine whether the bonding in a particular region is metallic, covalent-like, or weak, ${ }^{340}$ or whether in a certain region there is chemical bonding or a decaying electron density tail ${ }^{341}$ (see also ref. 293 and ref. 299). This can be done on the basis of a so-called inhomogeneity parameter $\alpha,{ }^{340}$ which is related to the electron localization function (ELF) ${ }^{342} \eta$ through $\eta=1 /(1+$ $\left.\alpha^{2}\right),{ }^{343}$ where $\eta$ can be used to rigorously classify chemical bonds, as noted by Sun et al. ${ }^{340}$ An empirical mGGA based on this principle, which has been trained on heats of formation and gas phase reaction barrier heights, is the MS2 DF. ${ }^{344}$ The RTPSS $\mathrm{DF}^{307}$ is a constraint-based mGGA DF in which one constraint was deliberately omitted to get a better description of chemisorption energies. Examples of semi-empirical mGGA DFs include M06-L, ${ }^{345}$ which was trained to describe maingroup thermochemistry, TM bonding, thermochemical kinetics, and non-covalent interactions, and $\mathrm{mBEEF},{ }^{305}$ which was trained to describe molecular formation and gas phase reaction energies, chemisorption energies, and solid state energetic and structural data. Examples of semi-empirical meta-NGAs are the MN12-L ${ }^{346}$ and MN15-L $\mathrm{L}^{32}$ DFs, which were both trained on extensive data sets including gas phase $E_{\mathrm{b}}$ and solid state lattice constants. Finally, the $\mathrm{B} 97 \mathrm{M}-\mathrm{V}^{347}$ and the SCAN/rVV10 $0^{330,339}$ DFs are examples of mGGAs incorporating van der Waals correlation DFs. Of these, the B97M-V DF is a semi-empirical DF trained on a large dataset concerning main group chemistry.

We now turn to the performance of the rung 3 DFs on the databases considered here. Of the non-empirical mGGAs, revTPSS is a somewhat better than the PBE GGA DF but tends to be worse than the GGA RPBE DF for gas phase barriers (Table 1), and revTPSS is worse than the GGA RPBE DF for chemisorption energies (Table 2). The SCAN DF does not improve over revTPSS for gas phase reaction barriers (Table 1), and is even worse for adsorption energies (Table 2). Adding more constraints has therefore not resulted in improved performance for these properties. In contrast, RTPSS shows a better performance on chemisorption energies than revTPSS. The MS2 DF does perform considerably better than all considered GGAs and NGAs for gas phase reaction barriers, but it is still outperformed by the RPBE DF for chemisorption energies. We also note that the semi-empirical BEEF-vdW GGA DF with van der Waals correlation DF shows a much better performance than MS2 on the SBH10 database for DC barriers, and on the CE26 chemisorption energy database (Table 3). Of the semi-empirical mGGAs and meta-NGAs, MN12-L and MN15-L show an excellent performance on the B76 database for gas phase reactions. These two DFs, and the mGGA DF B97M-V also containing non-local dispersion, also show a good performance for the larger DBH206 database. It should be interesting to test these DFs on DC reactions. These three DFs slightly outperform the semi-empirical mBEEF mGGA on gas phase $E_{\mathrm{b}}$. MN15-L and MN06-L both perform reasonably well for the $\mathrm{CE} 39 \mathrm{~b}$ database (chemisorption and physisorption on metals), although BEEF-vdW does slightly better. We note that adding $\tau$ in semi-empirical DFs has allowed bringing down the MUE for the B76 database for gas phase reactions from $5.3 \mathrm{kcal} \mathrm{mol}^{-1}$ for the best semi-empirical NGA to $1.7 \mathrm{kcal} \mathrm{mol}^{-1}$ for the MN15-L DF (Table 1), but that such a low MUE has yet to be realized for DC reactions (Table 3).

2.1.5. Rung 4: DFs based on exact exchange. Before we turn to the hybrid DFs of rung 4, we first provide a brief discussion of a second shortcoming of semi-local DFs (we already mentioned the problem that they are not able to describe the van der Waals attractive or dispersion interaction). The second problem concerns the so-called self-interaction, which may be discussed with reference to the Hartree-Fock (HF) description of the one-electron hydrogen atom (we follow ref. 31 in the description of this problem). HF theory is exact for the $\mathrm{H}$-atom, because the classical Coulomb and the non-classical exchange contributions to the electron-electron interaction cancel, as they should for a one-electron atom. In other words, HF oneelectron theory is free of self-interaction error (SIE). In semilocal DFT there is no exact exchange term, and the exchange needs to be described within the framework of the exchangecorrelation (XC) term. As a consequence, most semi-local DFs are not SIE free.

The pragmatic solution has been to mix in a fraction $X$ of $\mathrm{HF}$ exchange in the exchange term in the XC DF, and use a fraction of $(1-X)$ semi-local exchange ${ }^{293,299,347}$ which brings us to the hybrid DFs on rung 4. Hybrid DFs include an exact exchange (also often called HF exchange) component, and one could say that the extra ingredient added is exact exchange, or, alternatively, the occupied Kohn-Sham orbitals needed to compute it (see also below). Often the term exact exchange (rather than $\mathrm{HF}$ exchange) is used to avoid the impression that the exact exchange energy should equal the exchange energy in $\mathrm{HF}$ theory. ${ }^{293}$ To make this clearer, and to prepare the ground of the description of range-separated hybrid DFs, we first provide the expressions used for the exact exchange energy, which for real orbitals reads: ${ }^{293}$

$$
E_{\mathrm{X}}=\int \mathrm{d} \mathbf{r} \int \mathbf{r}^{\prime} \frac{\left|\rho_{\mathrm{X}}\left(\mathbf{r}, \mathbf{r}^{\prime}\right)\right|^{2}}{\left|\mathbf{r}-\mathbf{r}^{\prime}\right|}
$$

with

$$
\rho_{\mathrm{X}}=\sum_{1=1}^{N_{\text {occ }}} \phi_{i}(\mathbf{r}) \phi_{i}\left(\mathbf{r}^{\prime}\right) .
$$

In eqn (1b), $\phi_{i}(\mathbf{r})$ is the Kohn-Sham (KS) orbital $\mathrm{i}$ evaluated at the point $\mathbf{r}$ in $3 \mathrm{D}$ space. KS orbitals $\phi_{i}(\mathbf{r})$ differ from HF orbitals $\psi_{i}(\mathbf{r})$ because the $\psi_{i}(\mathbf{r})$ orbitals are evaluated in a formalism in which correlation is absent. As a result the exact exchange will usually not equal the exchange energy computed in HF theory. 
According to Perdew, exact exchange is needed to describe stretched bonds, as the XC hole can spread out over two or more atomic centers, thereby requiring a non-local description. ${ }^{299}$ As can be seen from eqn (1), the evaluation of exact exchange requires a double integral over $3 \mathrm{D}$ space, and the DFs of rung 4 are therefore called non-local (NL). As a result, the DFT calculation of a PES or DFMD calculations using hybrid DFs for a molecule interacting with a metal surface are at present usually considered computationally intractable. We will nevertheless provide a brief discussion here, as researchers are starting to use hybrid DFs in electronic structure calculations on molecules interacting with metal surfaces in the hope of correcting at least partially for the SIE (see e.g. ref. 348-350), and a screened hybrid DF has already been used in dynamics calculations on $\mathrm{O}_{2}+\mathrm{Al}(111)^{351}$ (see below).

The B3LYP DF is the best known global hybrid $\mathrm{DF}^{352}$ in which the exchange energy is a mixture of exact exchange $(20 \%)$ and GGA exchange, ${ }^{313}$ and GGA correlation ${ }^{353}$ (the DF is therefore called a hybrid GGA DF). By "global" we mean that $X$ is taken constant and not switched off depending on the distance $\left|\mathbf{r}-\mathbf{r}^{\prime}\right|$ in eqn (1a). ${ }^{293,354}$ The MO8-HX ${ }^{355}$ DF is a global hybrid mGGA DF, which combines a constant fraction of exact exchange with mGGA exchange. This DF was fitted to databases regarding main-group thermochemistry, chemical kinetics, and non-covalent interactions.

Hybrid DFs in which $X$ does depend on $\left|\mathbf{r}-\mathbf{r}^{\prime}\right|$ are called range-separated hybrid (RSH) DFs. ${ }^{293,354}$ Here, an important distinction needs to be made. In one type of $\mathrm{RSH} \mathrm{DF}$, the fraction $X$ of exact exchange decreases to zero at large distances between two points in 3D space. Such DFs are often called screened hybrid (SH) DFs. ${ }^{293}$ Examples of $\mathrm{SH}$ DFs are the HSE06 DF, ${ }^{356}$ which is a SH GGA DF, and the MN12-SX DF, ${ }^{357}$ which is a SH mNGA DF. Using completely screened exact exchange at large distances is appropriate when dealing with metals, which have an infinite dielectric constant. ${ }^{293}$ For these DFs an additional advantage is that the use of screened exact exchange is computationally favorable in plane wave DFT codes. ${ }^{356}$ In the other type of RSH DFs, $X$ increases with distance between the electrons. This has the advantage that the exchange DF becomes one-electron SIE free in the long range. ${ }^{354}$ These RSH DFs are often called long range corrected range separated hybrid DFs (abbreviated LCH DFs here). The recent $\omega \mathrm{B} 97 \mathrm{M}-\mathrm{V} \mathrm{DF}{ }^{354}$ is an example of a LCH mGGA DF (mixing exact exchange with semi-local mGGA exchange at long range), which also incorporates a van der Waals correlation DF. The vdW $1^{326}$ and vdW2 $2^{327}$ van der Waals correlation DFs have also been combined with hybrid exchange DFs. ${ }^{358}$

The global hybrid GGA B3LYP DF performs reasonably well on gas phase $E_{\mathrm{b}}$ (Table 1), but note that its performance on the corresponding databases is not better, and in some cases poorer, than that of the best mGGA DFs. The global hybrid mGGA DF M08-HX already performs much better: on the basis of its MUE, it shows chemical accuracy for the B76 database, and was in the top 3 list of performers on the DBH206 database (see Table 1 for its RMSE). The M08-HX DF contains a large fraction of exact exchange $(52.23 \%)$, which is useful for a good description of gas phase $E_{\mathrm{b}} \cdot{ }^{31}$ The rung $4 \mathrm{DF}$ that performed best on the DBH206 database is the LCH mGGA $\omega B 97 \mathrm{M}-\mathrm{V}$ DF (Table 1), which also incorporates a van der Waals DF. However, we note that it may well be that none of these DFs is very useful for the description of molecules interacting with metals, as these hybrid DFs all contain long range exact exchange.

The SH GGA DF considered here, HSE06, ${ }^{356}$ has a maximum of $25 \%$ exact exchange. Its performance on the B76 gas phase reaction barrier database is similar to that of B3LYP (Table 1). The SH mNGA DF considered here, MN12-SX, has a maximum of 25\% exact exchange like HSE06. However, MN12-SX clearly outperforms HSE06 on gas phase $E_{\mathrm{b}}$, possessing almost chemical accuracy for the B76 database (Table 1). MN12-SX also shows excellent accuracy on solid state physics properties, with a performance for solid state lattice constants (including metals) that is as good as that of the PBEsol DF. ${ }^{357}$

The performance of HSE06 on chemisorption energies (the CE26 database) is rather disappointing, with GGA and GGA van der Waals DFs like BEEF-vdW, RPBE, and even PBE outperforming the HSE06 DF (Tables 2 and 3). The HSE06 DF systematically underestimates both $E_{\mathrm{b}}$ for DC and chemisorption energies (Table 3). The HSE06 DF is outperformed for DC barriers by the semi-local BEEF-vdW and MS2 DFs (Table 3).

In ref. 301 only one GGA DF with NL correlation, one mGGA, and one SH DF were tested on the SBH10 database for dissociation barriers on metals. On the basis of this limited test, it is too early to draw conclusions on which class of DFs should be best for the description of reactions on metals. For instance, we would very much like to see results for the MN12-SX DF, given its excellent performance on gas phase $E_{\mathrm{b}}$ as well as lattice constants. If this $\mathrm{SH}$ DF would also systematically underestimate DC barriers, one might try fitting a SH DF with a larger fraction of short-range exact exchange. Ref. ref. 301 noted that the performance of the tested DFs mirrored that on chemisorption energies, and attributed this to most of the TSs present in SBH10 having "late barriers", resembling final states. It is however noteworthy that the semi-local GGA with NL van der Waals correlation does not systematically underestimate DC barriers on metals (Table 3), while it does systematically underestimate the gas phase reaction barriers in the $\mathrm{DBH} 24 / 08$ database, similar to the PBE and RPBE semi-local GGAs (see Table 1). This point is relevant to the construction of SRP-DFs, and we will get back to it below.

2.1.6. Rung 5 DFs and wave function theory. We finally come to the DFs on rung $5 .^{299}$ DFs on this rung also use virtual Kohn-Sham orbitals (the extra ingredient added, Fig. 4) to compute correlation energies with a formalism that is NL in the orbitals, as opposed to methods that are NL in the densities, such as the van der Waals correlation DFs. ${ }^{293,299}$ This makes rung 5 DFs very expensive to use. A well-known example of rung 5 DFs is the random phase approximation (RPA), ${ }^{359-362}$ which uses many-body perturbation theory for correlation in combination with exact exchange, and, according to Truhlar and co-workers, may therefore be considered as exchange-free perturbation theory. ${ }^{293}$ Rung 5 DFs may also be mixtures of hybrid exchange DFs with wave function theory (WFT) for correlation, which are 
also referred to as double hybrids. ${ }^{363}$ The RPA method was tested on the B76 database for gas phase reaction barriers, for which it showed a very good performance (MUE of $2.3 \mathrm{kcal} \mathrm{mol}^{-1}$ ), but not yet chemical accuracy. The RPA method was also tested on the CE10 database for chemisorption to metals, and was shown to have a performance intermediate to RPBE and BEEF-vdW for this database ${ }^{306}$ (Table 2).

On the basis of the rather large MUE calculated with the RPA for the CE10 $\left(4.8 \mathrm{kcal} \mathrm{mol}^{-1}\right)^{306}$ and the B76 database (MUE = $\left.2.3 \mathrm{kcal} \mathrm{mol}^{-1}\right),{ }^{364}$ we would argue that the authors ${ }^{306}$ have not yet shown enough evidence that the theoretical method considered by them (i.e., the RPA method) rather than experiment can be used as a benchmark to test other theoretical methods against for their accuracy for chemisorption. This situation is different for gas phase reaction barriers, with the $\operatorname{CCSD}(\mathrm{T})$ method (a fifth order perturbation WFT method) exhibiting chemical accuracy for the DBH24/08 database (Table 1).

A few comments regarding the performance of hybrid DFT and WFT on gas phase reaction barriers are in order. As can be seen from Table 1, HF systematically overestimates gas phase $E_{\mathrm{b}}$, while the LDA systematically underestimates them. This may be used as an empirical argument for mixing in exact exchange in DFT. ${ }^{31}$ Second, it is useful to consider the accuracy of the MP2 method, as it has been implemented in several periodic codes, e.g. the CRYSTAL code. ${ }^{365}$ While MP2 clearly improves upon the HF method for the DB24 database, it still considerably and systematically overestimates gas phase reaction barriers (MSE $=4.7 \mathrm{kcal} \mathrm{mol}^{-1}$ ), making it unlikely that periodic MP2 calculations will be able to deliver chemical accuracy for $E_{\mathrm{b}}$ for DC on metals.

2.1.7. Computational points and benchmarking. We finish this subsection with two computational points and a general comment regarding benchmarking. First, it is important to note that results of calculations with semi-empirical mGGA or hybrid mGGA DFs may be quite sensitive to the integration mesh used in the calculations. A problem of some of the Minnesota DFs is that potential energy curves for weak noncovalent interactions may display oscillatory behavior, ${ }^{366}$ and this may carry over to van der Waals interactions in systems where a molecule interacts with a metal at long range. Unfortunately this is a problem with the MN12-SX DF, which also tends to perform less well for non-covalent interactions in general. These are real drawbacks, as it would obviously be advantageous to have a good $\mathrm{SH}$ meta-gradient approximation DF available that could outperform HSE06 on molecules interacting with metals. Second, when working with the Lundqvist-Langreth van der Waals correlation DFs ${ }^{326,327}$ one should be aware that some observables or properties may be quite sensitive to the pseudopotential used. ${ }^{302}$

The databases being developed for adsorption to metals and dissociation barriers on metals are obviously useful for benchmarking DFT for these interactions. In a recent paper Bligaard et $a .^{367}$ have discussed best practices for benchmarking in catalysis science. In this context, and after reviewing the work on the databases referenced here, we note that, while it may be useful to make small extensions to databases (e.g. from $\mathrm{CE} 39^{303}$ to $\mathrm{ADS} 41^{302}$ ) or change a reference (e.g. to chemisorption of fragments resulting from dissociation ${ }^{368}$ ), better comparability with earlier work can be achieved if the results for the earlier smaller database or reference are also presented.

\subsection{The specific reaction parameter approach to DFT (SRP-DFT)}

The idea of using a specific reaction parameter (SRP) or a few SRPs with electronic structure theory can be traced back to work of Truhlar and co-workers. ${ }^{369}$ Their work was driven by the wish to extend semi-empirical approaches to larger systems. For a small system a semi-empirical approach can be implemented by fitting an analytical PES and tuning one or more PES parameters to fit to, for instance, a measured rate constant; however, such an approach was deemed impractical for larger systems. Instead, the idea was to use an electronic structure method as a fitting tool, by adjusting one or more of its parameters (the SRPs) to reproduce, for instance, a rate constant. As stated by Truhlar and co-workers, ${ }^{369}$ the interest in the resulting method was then not so much its ability to reproduce the rate constant it was fitted to, but rather the resulting method's usefulness for studying details of dynamical processes, or the effect of solvation on the reaction dynamics. The authors originally used an AM1 model and modified specific one-electron energies (which are parameters in the AM1 model) to obtain agreement with experiment. They also recognized that obtaining a reasonably accurate, but computationally inexpensive method allowed them to perform direct dynamics calculations. Subsequently, calculations with semi-empirical electronic Hamiltonians using SRPs were performed for a range of applications, ${ }^{370-375}$ including an application to surface reaction dynamics by the Hase group (to the reaction of O-atoms with an alkyl thiolate self-assembled monolayer). ${ }^{376}$

Truhlar and co-workers were also the first to realize that the concept of SRPs could be applied with DFT, by fitting one or more of the coefficients occurring in a DF to a measured observable (again, usually a rate constant). ${ }^{42}$ In their first application of SRP-DFT, they varied two parameters of a three-parameter hybrid $\mathrm{DF}$ due to Becke to obtain a good fit to the rate constant for the gas-phase reaction of $\mathrm{H}$ with $\mathrm{CH}_{3} \mathrm{OH}$, and used this DF in direct dynamics studies of this reaction in the condensed phase. ${ }^{42}$ Later applications of SRP-DFT concerned gas phase reactions. ${ }^{377-380}$ While these early studies all changed the parameter $X$ in global hybrid DFs to obtain better agreement with experiments on reactions, a recent study on the $\mathrm{H}+\mathrm{H}_{2}$ reaction obtained excellent agreement with experiments by fine-tuning specific parameters of a double hybrid DF. ${ }^{381}$

The SRP-DFT approach was first applied to a moleculemetal surface reaction in $2009 .{ }^{43}$ Contrary to the treatment of gas phase reactions, in the applications of SRP-DFT to molecule-metal surface reactions hybrid DFs have not yet been used. Reasons for this include the computational expense of plane wave DFT calculations using hybrid DFs, and the tendency of hybrid DFs to overestimate the bandwidth of the highest metal band. ${ }^{348,349}$ Instead, the SRP-DFs that have been used to study reactions of molecules with metal surfaces have mostly involved 
weighted averages of GGA DFs, or weighted averages of GGA exchange DFs with NL correlation DFs added. Specifically, the following generic expressions have been used:

$$
\begin{gathered}
E_{\mathrm{XC}}^{\mathrm{SRP}}=\alpha E_{\mathrm{XC}}^{\mathrm{GGA} 1}+(1-\alpha) E_{\mathrm{XC}}^{\mathrm{GGA} 2}(\text { ref. } 243) \\
E_{\mathrm{XC}}^{\mathrm{SRP}}=\alpha E_{\mathrm{X}}^{\mathrm{GGA} 1}+(1-\alpha) E_{\mathrm{X}}^{\mathrm{GGA} 2}+E_{\mathrm{C}}^{\mathrm{GGA}}(\text { ref. } 175) \\
E_{\mathrm{XC}}^{\mathrm{SRP}}=\alpha E_{\mathrm{X}}^{\mathrm{GGA} 1}+(1-\alpha) E_{\mathrm{X}}^{\mathrm{GGA} 2}+E_{\mathrm{C}}^{\mathrm{NL}}(\text { ref. } 44) \\
E_{\mathrm{XC}}^{\mathrm{SRP}}=E_{\mathrm{X}}^{\mathrm{PBE} \alpha}+E_{\mathrm{C}}^{\mathrm{NL}}(\text { ref. } 156)
\end{gathered}
$$

Also, for $\mathrm{H}_{2}+\mathrm{Cu}(111)$ it has been established that an SRP-DF can be constructed of the form

$$
E_{\mathrm{XC}}^{\mathrm{SRP}}=\alpha E_{\mathrm{X}}^{\mathrm{mGGA} 1}+(1-\alpha) E_{\mathrm{X}}^{\mathrm{mGGA} 2}+E_{\mathrm{C}}^{\mathrm{mGGA}}(\text { ref. 152). }
$$

A basic idea of all of these DFs is that the parameter $\alpha$ should be tunable so that, with an appropriate dynamical model, dynamics calculations using a PES or forces computed directly with the SRP-DF can accurately reproduce measured $S_{0}$ curves. In the above equations, this was achieved by taking a weighted average of two GGA XC DFs (eqn (2a)), by taking a weighted average of two GGA exchange DFs and using the GGA correlation DF shared by the corresponding GGAs (eqn (2b)) (for example, $\mathrm{PBE}^{195}$ and RPBE, ${ }^{196}$ which share the PBE correlation DF), by taking a weighted average of two GGA exchange DFs and a NL correlation DF like vdW1 ${ }^{326}$ (eqn (2c)), by combining a tunable exchange $\mathrm{DF}\left(\mathrm{PBE} \alpha^{382}\right)$ with a NL correlation $\mathrm{DF}\left(\mathrm{vdW} 2,^{327}\right.$ eqn (2d)), or by taking a weighted average of two mGGAs exchange DFs and using the mGGA correlation DF shared by the corresponding mGGAs ${ }^{152}$ (eqn (2e)). When employing one of the eqn (2a)-(2c) and (2e), it is desirable that using one of the DFs effectively used leads to consistent overestimation of the measured $S_{0}$ curve, while the other DF consistently underestimates the measured $S_{0}$. Note that the PBE $\alpha^{382} \mathrm{DF}$ interpolates between a DF that closely resembles the WC $\mathrm{DF}^{197}(\alpha=0.52)$, the $\operatorname{PBE} \operatorname{DF}^{195}(\alpha=1)$ and the RPBE $\operatorname{DF}^{196}(\alpha=\infty)$.

The inspiration to take the first SRP-DF according to eqn (2a) came from a comparison ${ }^{200}$ of experimental $S_{0}$ with theoretical $S_{0}$ previously computed with the PW91 and RPBE DFs for $\mathrm{H}_{2}+\mathrm{Ru}(0001)$, from work on ammonia production, ${ }^{6}$ and from research on error estimation in DFT. ${ }^{383}$ The work on $\mathrm{H}_{2}+\mathrm{Ru}(0001)^{200}$ showed that, to a reasonable approximation, the experimental $S_{0}$ fell in between reaction probabilities predicted ${ }^{384}$ with the PW91 and RPBE DFs. Fig. 6 illustrates the principle for $\mathrm{H}_{2}+\mathrm{Cu}(111)$ : QCT calculations based on the PW91 DF (the RPBE DF) overestimated (underestimated) measured values of $S_{0}$. However, $S_{0}$ calculated effectively with a DF that can be written as $E_{\mathrm{XC}}^{\mathrm{SRP}}=0.57 E_{\mathrm{XC}}^{\mathrm{PW} 91}+0.43 E_{\mathrm{XC}}^{\mathrm{RPBE}}$ were in excellent agreement with experiment, as further discussed below. The work on $\mathrm{NH}_{3}$ production computed production rates as a function of stationary point energies interpolated between PW91 and RPBE values, showing that error cancellation between PW91 and RPBE rates occurred due to a compensation effect. ${ }^{6}$ The research on error estimation in $\mathrm{DFT}^{383}$ showed that a semiempirical GGA DF optimized by fitting molecular atomization energies and cohesive energies had an exchange enhancement factor intermediate between PBE and RPBE.

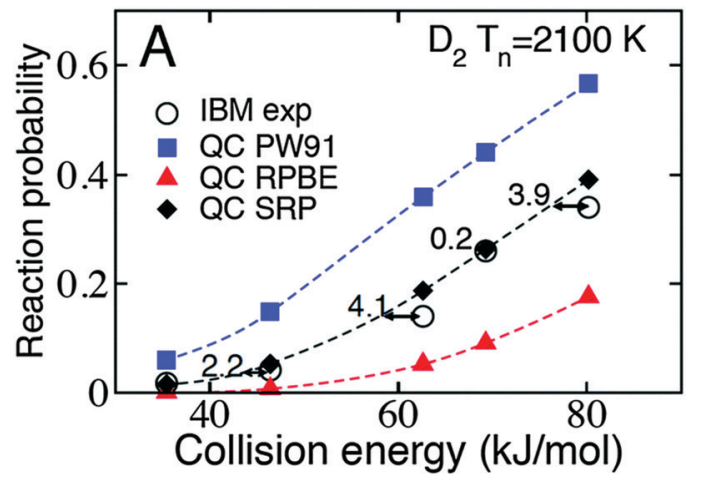

Fig. $6 S_{0}$ computed $^{43}$ with the QCT method ( $Q C$ in legend) using the PW91, ${ }^{312}$ RPBE, $^{196}$ and SRP43 ${ }^{43}$ (SRP) DFs are compared with experimental ${ }^{12}$ values for $D_{2}+C u(111)$ (IBM exp). The computed $S_{0}$ exhibit statistical errors $\leq 0.005$. Figure taken from ref. 43 .

Because SRP-DFT operates essentially by computing $S_{0}$ curves and comparing with experimental $S_{0}$ curves, great care has to be taken to model the experiment as accurately as possible. In principle this requires knowledge about the MBs used in the experiments. As further discussed below, these can be characterized by the vibrational temperature $\left(T_{\mathrm{vib}}\right)$ of the molecules in the beam (usually equal to the nozzle temperature, $T_{\mathrm{N}}$ ), the rotational temperature $\left(T_{\text {rot }}\right)$ in the beam (details below), and the distribution of the velocities or the $E_{i}$ in the direction towards the surface. As will also be discussed below, experiments greatly differ in terms of the amount of details in which these beam properties are documented. Obviously, the success of fitting an SRP-DF for a particular system will depend to a large extent on the availability of this detailed information. Sometimes this involves much guesswork, ${ }^{156}$ in other cases very detailed information is available on experiments done quite some time ago $^{43}$ or velocity distributions can be fitted to published time-of-flight (TOF) distributions of $\mathrm{MBs},{ }^{43}$ and in other cases the information becomes available through a joint theoretical-experimental effort aimed partly at deriving an SRP-DF for the system investigated. ${ }^{44,47}$ For the latter case, guidelines have been presented for the design of experiments to be modeled with DFMD calculations aimed at developing an SRP-DF ("surface reaction barriometry",47).

\subsection{Tight binding methods based on DFT}

To represent the interaction of $\mathrm{H}_{2}$ with $\mathrm{Pd}(100)^{385}$ and later of $\mathrm{O}_{2}$ with Pt(111) Groß and co-workers ${ }^{386,387}$ have used a total energy tight-binding (TETB) method that was developed especially for metals by Papaconstantopoulos and co-workers. ${ }^{388,389}$ With this TTEB method, and after some fitting, they could reproduce a set of previously computed PW91 DFT energies for $\mathrm{H}_{2}+\mathrm{Pd}(100)$ with an RMSE of about $100 \mathrm{meV}$, and the TTEB results were of higher accuracy in regions where this was needed. ${ }^{385}$ A similarly low RMSE was reported for $\mathrm{O}_{2}+\mathrm{Pt}(111) .{ }^{387}$ The TTEB method could subsequently be used in direct dynamics calculations where the number of trajectories that could be computed was high enough to allow important new conclusions regarding a seeming paradox concerning experimental findings on sticking and scattering, 
on the basis of statistically relevant results. ${ }^{387}$ At the time, this could not have been achieved with DFMD calculations.

The TTEB method ${ }^{388,389}$ used by Groß and co-workers bears some similarities with the more generally known density functional tight-binding (DFTB) method, ${ }^{390}$ but it is not equal to it, and in some sense the TTEB method resembles a fitting method, albeit that physical meaning can be attached to its parameters. ${ }^{385}$ We are not aware of applications to moleculemetal surface reactions after 2006. To our knowledge, DFTB ${ }^{390}$ has not been applied to molecule-metal surface reactions; however, it has been applied to large molecules, clusters and nonoparticles, including metal clusters, metal clusters interacting with molecules (functionalized clusters), adsorption of molecules on metaloxide surfaces, etc. For a recent review see ref. 390.

\subsection{Other electronic structure methods for molecules interacting with metal surfaces}

Researchers are increasingly looking at applying methods to molecules interacting with metal surfaces of which the accuracy and reliability may surpass that of DFT with the best DFs in future, or already surpasses DFT, but which allow only a few single point calculations rather than the mapping out of a complete PES. Carter and coworkers have developed a density functional embedded correlated wave function theory (the ECW method) that is potentially very accurate for molecules interacting with metals. ${ }^{391,392}$ In this method, the system is partitioned into a cluster (containing the adsorbing molecule and a cluster of metal atoms near to it) and a (possibly periodic) bulk environment. An assumption made is that these subsystems are non-degenerate, and for this case a unique global embedding potential can be defined such that the embedding potential for the cluster equals the embedding potential for the bulk environment. The total energy of the system $E_{\text {tot }}$ is calculated from the energy of the total system computed with DFT $E_{\text {tot }}^{\mathrm{DFT}}$, the energy of the embedded cluster computed with a high level $a b$ initio (or correlated wave function (CW)) method $E_{\mathrm{emb}, \mathrm{cl}}^{\mathrm{CW}}$, and the energy of the embedded cluster computed with DFT $E_{\mathrm{emb}, \mathrm{cl}}^{\mathrm{DFT}}$ as follows: ${ }^{392}$

$$
E_{\mathrm{tot}}=E_{\mathrm{tot}}^{\mathrm{DFT}}+\left(E_{\mathrm{emb}, \mathrm{cl}}^{\mathrm{CW}}-E_{\mathrm{emb}, \mathrm{cl}}^{\mathrm{DFT}}\right) .
$$

The underlying assumption is that the CW correction to the energy of the cluster improves the total energy computed for the total system relative to the DFT results. The scheme does not consider changes in the environment due to treating the embedded cluster with a CW method, but the assumption is made that this is not problematic due to the short screening length in metals. ${ }^{392}$ Also, the method can be extended to lift the limitation that the subsystems should be non-degenerate. ${ }^{393}$

CW methods that have been used in calculations on electronic ground state systems consisting of a molecule interacting with a metal surface include CASSCF, ${ }^{394}$ CASPT2, ${ }^{395,396}$ and CIS (configuration interaction singles, or CI singles). ${ }^{397}$ Applications of the ECW method to such systems include calculations on $\mathrm{H}_{2}+$ $\mathrm{Au}(111)^{398}$ and on $\mathrm{O}_{2}+\mathrm{Al}(111) \cdot{ }^{35,399,400}$ In a recent successful application, QCT calculations on the ECW PES were able to reproduce experiments on the sticking of $\mathrm{O}_{2}$ on $\mathrm{Al}(111)$ semiquantitatively (albeit not yet with chemical accuracy, see Fig. 7). ${ }^{35}$

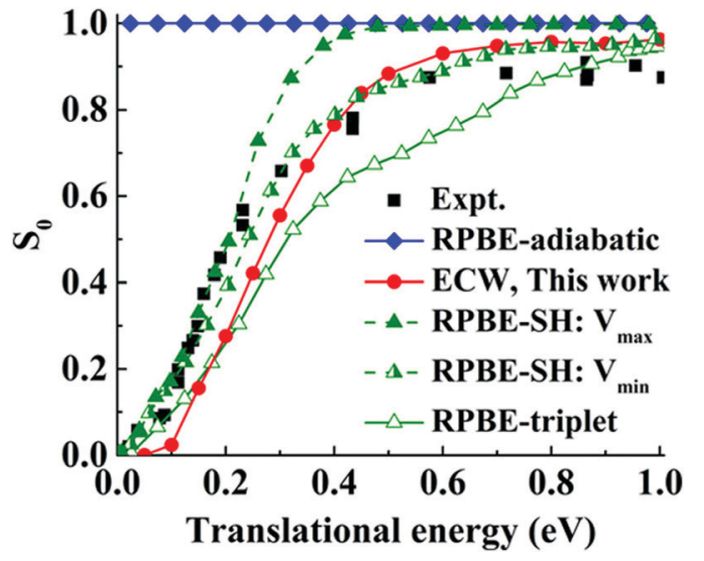

Fig. $7 S_{0}$ computed with the QCT method using an adiabatic RPBE ${ }^{196}$ PES, ${ }^{570}$ a triplet RPBE PES computed with spin-constrained DFT, ${ }^{570}$ a triplet and singlet RPBE PES with surface hopping using maximal $\left(V_{\max }\right)$ and minimal $\left(V_{\text {min }}\right)$ coupling, ${ }^{573}$ and an ECW FPLEPS PES ${ }^{35}$ are compared with experimental $S_{0}{ }^{217}$ Figure taken from ref. 35 (https://pubs.acs.org/ doi/10.1021/acs.jpclett.8b01470). Further permission requests to be directed to the ACS.

In the work on $\mathrm{O}_{2}+\mathrm{Al}(111)$, in the construction of the PES the CASPT2 $\operatorname{method}^{395,396}$ was used in the calculations on the embedded cluster. The ECW method can also be applied to excited states of systems consisting of a molecule interacting with a metal surface. ${ }^{398,401-404}$ It can be expected that as computers become more powerful the ECW approach will eventually achieve chemical accuracy for barriers for molecule-metal surface reactions, if large enough clusters can be treated with a high enough level CW method.

With the ONIOM approach ${ }^{405}$ one can compute an improved total energy of a system in which a molecule interacts with a metal surface using an equation similar to eqn (3), i.e.,

$$
E_{\mathrm{tot}}^{\mathrm{ONIOM}}=E_{\mathrm{tot}}^{\mathrm{LL}}+\left(E_{\mathrm{cl}}^{\mathrm{HL}}-E_{\mathrm{cl}}^{\mathrm{LL}}\right) .
$$

In eqn (4), "LL" stands for a low-level method, and "HL" stands for a high-level, i.e., more accurate method. A difference with the ECW method is that calculations on bare clusters are done, without the presence of an embedding potential representing the effect of the environment. This may adversely affect the accuracy for molecules interacting with metals, as the delocalized Bloch states found in bulk metal are quite different from the wave functions describing metal atoms in clusters. ${ }^{392}$ The ONIOM approach has nevertheless been used to compute $E_{\mathrm{b}}$ for $\mathrm{H}_{2}$ dissociation over a surface atom in $\mathrm{Cu}(100),{ }^{406}$ using PBE-DFT as the LL method and both $\operatorname{CCSD}(\mathrm{T})^{407}$ and MRCI $+\mathrm{Q}^{408}(+\mathrm{Q}$ stands for the Davidson correction ${ }^{409}$ ) as the HL method. The ONIOM calculation with MRCI $+\mathrm{Q}$ was in excellent agreement $\left(E_{\mathrm{b}}=0.873 \mathrm{eV}\right)$ with the semi-empirical SRP-DFT result for $\mathrm{H}_{2}+$ $\mathrm{Cu}(100)\left(E_{\mathrm{b}}=0.87 \mathrm{eV}\right)$, but the ONIOM calculation with $\operatorname{CCSD}(\mathrm{T})$ $\left(E_{\mathrm{b}}=0.629 \mathrm{eV}\right)$ was not. Calculations with the ONIOM approach have also addressed the physisorption of $\mathrm{H}_{2}$ on $\mathrm{Cu}(100) .{ }^{410}$ These calculations employed the vdW2 $\operatorname{method}^{327}$ as the LL method, and the $\operatorname{CCSD}(\mathrm{T}) \operatorname{method}^{407,411}$ as the HL method. Transition energies between physisorbed states were in very good 
agreement with experiment, and the agreement was somewhat better than obtained using a bare cluster of $\mathrm{Cu}$ atoms modeling the $\mathrm{Cu}(100)$ surface with $\operatorname{CCSD}(\mathrm{T}) .{ }^{410}$ The above results suggest that the ONIOM approach may work quite well for specific cases of chemisorption and physisorption, but more tests are needed to confirm this.

The method that is considered to be the "gold standard" for gas phase chemistry, $\operatorname{CCSD}(\mathrm{T})$, is increasingly being used for materials science applications. ${ }^{412}$ A periodic $\operatorname{CCSD}(\mathrm{T})$ calculation has recently been performed on DC of $\mathrm{H}_{2}$ on a semi-conductor surface, i.e., $\mathrm{Si}(100) .{ }^{413}$ For this one system, barrier heights computed with $\operatorname{CCSD}(\mathrm{T})$ agreed with QMC results to within the stochastic error bar of the QMC results. Comparison was made with lower level CW approaches, and with results obtained with several DF functions. ${ }^{413}$ While the $\operatorname{CCSD}(\mathrm{T})$ method shows a high future promise for achieving chemical accuracy for molecules reacting on metals, an application of periodic $\operatorname{CCSD}(\mathrm{T})$ to a molecule reacting on a metal has yet to be demonstrated for a simple metal, let alone a TM.

Finally, the stochastic DMC method ${ }^{414,415}$ also shows considerable promise for achieving chemical accuracy for moleculemetal surface reactions. A nice property of DMC is that its computational cost scales favorably with the number of electrons $N\left(\right.$ as $\left.\mathrm{O}\left(N^{3}\right)\right)$ if localized orbitals are used to expand the electronic orbitals in. ${ }^{416,417}$ In contrast, $\operatorname{CCSD}(\mathrm{T})$ scales with $N$ as $\mathrm{O}\left(N^{7}\right) \cdot{ }^{37}$ In principle the DMC method is an exact method, ${ }^{415,416,418}$ and the parallelizability of DMC makes the method very suitable for highperformance computing: ${ }^{418}$ With DMC it is possible to reproduce gas phase $E_{\mathrm{b}}$ measured in experiments or computed with ab initio methods to within or close to chemical accuracy. ${ }^{419-424}$ The most extensive information comes from two recent papers, ${ }^{423,424}$ the results of which can be combined to compute the MUE for the B76 gas phase reaction barrier heights, which leads to a MUE value of $1.2 \mathrm{kcal} \mathrm{mol}^{-1}$; here a single determinant PBE trial wave function was used (see also Table 1). The largest error is usually made in the calculation of the transition state energy, and there is some correlation between the approximate nodal error and the HOMO-LUMO gap of the reactants. ${ }^{422,424}$ DMC calculations have been used earlier to study molecular adsorption of $\mathrm{CO}$ and $\mathrm{H}_{2} \mathrm{O}$ on $\mathrm{Cu}(100),{ }^{425}$ and $\mathrm{DC}$ of $\mathrm{N}_{2}$ on $\mathrm{Cu}(111)^{426}$ and of $\mathrm{H}_{2}$ on $\mathrm{Mg}(0001) .{ }^{427}$ Recent DMC calculations have reproduced semiempirical DC barrier heights for $\mathrm{H}_{2}+\mathrm{Cu}(111)^{34}$ and for $\mathrm{H}_{2}+$ $\mathrm{Pt}(111)$ to within $1.6 \pm 1.0$ and $0.9 \pm 1.0 \mathrm{kcal} \mathrm{mol}^{-1}$, respectively. Also, recent DMC calculations correctly predict the site-preference for $\mathrm{CO}$ adsorption on $\mathrm{Rh}(111), \operatorname{Ir}(111), \mathrm{Pt}(111)$, and $\mathrm{Cu}(111)$, which represents a challenging problem. ${ }^{428}$ An important recent result of DMC and DFT calculations on $\mathrm{H}_{2}+\mathrm{Al}(110)$ is that all eight tested functionals that were tested against DMC accurately describe the variation of the barrier height with impact site and molecular orientation $^{429}$ (see Fig. 8), which, as discussed later, is important to the correct description of sticking by SRP-DFT. Very recently DMC calculations have been published that very accurately (to within $1 \mathrm{~kJ} \mathrm{~mol}^{-1}$ ) reproduce the activation barrier for $\mathrm{H}_{2} \mathrm{O}$ addition of $\mathrm{H}_{2} \mathrm{O}$ to $\mathrm{CO}$ on $\mathrm{Pt}(111) .{ }^{430}$

In view of its recent successes, it is likely that DMC calculations start delivering chemical accuracy for reactions of molecules with
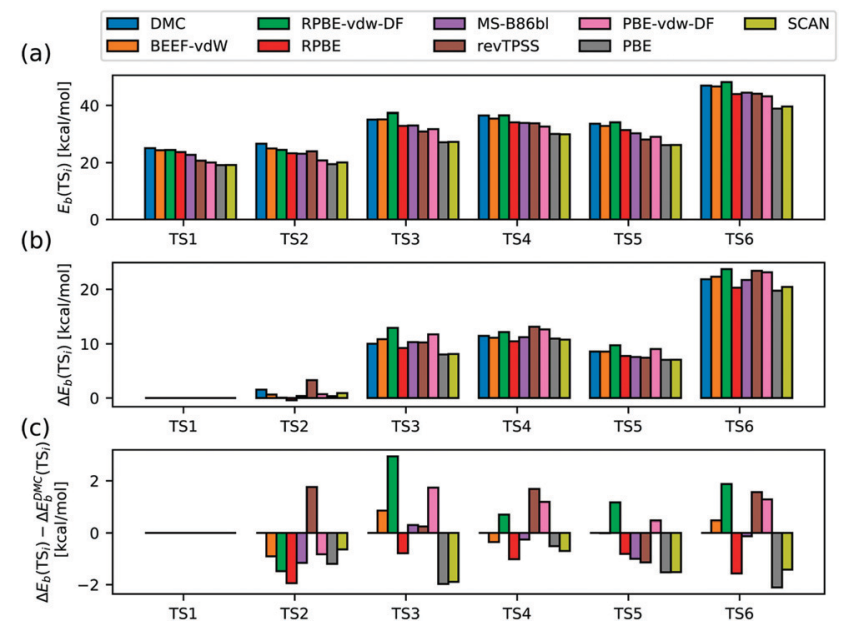

Fig. 8 (a) Comparison of barrier heights $E_{\mathrm{b}}$ computed ${ }^{429}$ for $\mathrm{H}_{2}+\mathrm{Al}(110)$, for six geometries (TS1-TS6) sampling different impact sites and orientations with DMC and DFT using 8 different density functionals. (b) Similar as in (a), but now plotting $\Delta E_{\mathrm{b}}(\mathrm{TS} i)=E_{\mathrm{b}}(\mathrm{TS} i)-E_{\mathrm{b}}(\mathrm{TS} 1)$ for $i=1-6$, for each method. (c) Similar to (b), but now plotting $\Delta E_{\mathrm{b}}\left(\mathrm{TS}\right.$ i) $-\Delta E_{\mathrm{b}}{ }^{\mathrm{DMC}}$ (TSi) for each method. Reprinted from [A. Powell, G. J. Kroes and K. Doblhoff-Dier, Quantum Monte Carlo calculations on dissociative chemisorption of $\mathrm{H}_{2}$ on Al(110): minimum barrier heights and their comparison to DFT values, J. Chem. Phys., 2020, 153, 224701], with the permission of AIP Publishing.

TMs in the not to distant future. Present sources of errors include the locality error (due to the need to use pseudo-potentials), finitesize errors, time-step errors, and fixed node errors due to the need to use an approximate wave function to start the calculations. ${ }^{34}$ It might be possible to reduce finite-size errors and locality errors by using density functional embedding of DMC. ${ }^{431}$ While this method has recently been shown to be applicable to a few systems, ${ }^{431}$ more work is needed to demonstrate its accuracy for molecules interacting with metals.

\section{PES fitting}

Dynamics calculations, which are needed to compute the reaction probabilities for comparison with MB sticking experiments, can be performed efficiently if a PES is available. Obtaining a PES requires interpolating or fitting to electronic structure data. Performing an accurate interpolation or fit of electronic structure data is obviously important: dynamics results obtained with a PES will only truly reflect the quality of the underlying electronic structure method if the energies obtained with it are accurately interpolated or fitted. Below, we describe several methods for fitting PESs that have been used in research on dynamics of reactions at metal surfaces.

\subsection{Neural network (NN) methods}

NN methods and other machine learning methods have been covered in a recent tutorial review ${ }^{432}$ and in recent perspectives $^{433-435}$ and reviews, ${ }^{436,437}$ and we borrow extensively from some of these papers ${ }^{432,433,436}$ in the below discussion. Artificial NNs may be defined as follows: "Artificial NNs are massively parallel interconnected networks of simple (usually adaptive) 
elements and their hierarchical organizations which are intended to interact with the objects of the real world in the same way as biological nervous systems do". ${ }^{438}$ In potential fitting, usually multilayer feed-forward (MLFF) NNs are used. MLFF NNs are "universal approximators", i.e., in principle they can be used to approximate unknown and arbitrary multidimensional functions to arbitrary accuracy when presented with a set of known function values (ref. 432 and references therein). An advantage of NN potentials (NNPs) is that no knowledge is needed regarding the functional form of the PES. ${ }^{432}$ A disadvantage is that considerable effort may have to be put into the "training" of the network. ${ }^{432}$

Essentially, for the most accurate PES fits, two types of NN methods are used in recent applications to gas-surface scattering. For molecules interacting with static surfaces, the permutation invariant polynomial NN (PIP-NN) approach ${ }^{439,440}$ can be used, and for molecules interacting with mobile surfaces, the highdimensional NN (HDNN) approach ${ }^{432,441-443}$ can be used.

In the PIP-NN approach to gas-surface scattering, ${ }^{439,440}$ polynomials are used that automatically incorporate the symmetry with respect to the permutation of the nuclei (PIPs). ${ }^{44-446}$ In the PIP-NN approach, in the first step the coordinates of the atoms in the molecule are transformed to the values taken on by polynomials incorporating the permutation symmetry. The values of these polynomials are then used as inputs to MLFF NNs. ${ }^{66}$ The method has been used to generate accurate fits for, for instance, $\mathrm{H}_{2}+\mathrm{Cu}(111)$ and $\mathrm{Ag}(111),{ }^{439} \mathrm{H}_{2}+\mathrm{Co}(0001),{ }^{104} \mathrm{H}_{2} \mathrm{O}+$ $\mathrm{Ni}(111),{ }^{121} \mathrm{CO}_{2}+\mathrm{Ni}(100),{ }^{92} \mathrm{CH}_{4}+\mathrm{Ni}(111),{ }^{167} \mathrm{NH}_{3}+\mathrm{Ru}(0001)^{66}$ and $\mathrm{CH}_{3} \mathrm{OH}+\mathrm{Cu}(111) .{ }^{144}$ The method allows accurate fits, for example, RMSEs of $2.5 \mathrm{meV}$ (using $\sim 4000$ points), and $14.7 \mathrm{meV}$ (using $\sim 18000$ points) have been reported for $\mathrm{H}_{2}+$ $\mathrm{Cu}(111)$ and $\mathrm{CO}_{2}+\mathrm{Ni}(100)$, respectively. ${ }^{440}$ A drawback of the method is that the number of PIPs grows fast with the number of DOFs in the system, making it hard to apply to complicated systems containing, for example, more than 10 atoms of the same element. ${ }^{447}$ Although this may be alleviated by removing redundant PIPs, ${ }^{448,449}$ this fast growth of the number of PIPs in practice limits its application to molecules interacting with static surfaces.

In early applications NN methods were applied to fitting PESs for molecules interacting with surfaces without using PIPs as input to the NN. ${ }^{82,450-454}$ NN methods that do not use PIPs as inputs are still used for fitting PESs for molecules interacting with static surfaces while achieving quite high accuracy. ${ }^{61,73,98,455-457} \mathrm{~A}$ special class of NN methods ${ }^{458-460}$ is constructed in such a way that the resulting PES has a sum of products form, which is advantageous when used in combination with specific quantum dynamical methods, as will be discussed below.

The HDNN approach ${ }^{432,441-443}$ can be used for molecules interacting with mobile surfaces. In this approach the total energy of the system is constructed as the sum of $N_{\text {atom }}$ atomic energy contributions, where $N_{\text {atom }}$ is the number of atoms in the system. Each atom belonging to a specific chemical element is described by the atomic NN for that element. These atomic NNs describe the energy of the atom as it depends on its local chemical environment to within a cut-off radius. This may involve the use of atom-centered symmetry functions ${ }^{442}$ describing two-body interactions depending on interatomic distances and three-body interactions depending on interatomic distances and the angle subtended by the three atoms whose interaction is described. ${ }^{442}$ Later, some minor modifications to these symmetry functions have been proposed. ${ }^{62,461}$ The values of the symmetry functions are taken as the input parameters to the atomic NNs. Advantages of the HDNN approach are that (i) the introduction of cut-offs, which define the size of the chemical environments of the atoms, limits the dimensionality of the fit, (ii) permutation symmetry is automatically obeyed, and (iii) the number of atoms of a specific element already contained in the system can in principle be changed without the need for retraining the NN. A fourth advantage will be mentioned below. The method has already been applied to the calculation of $S_{0}$ for a few systems, i.e., $\mathrm{N}_{2}+$ $\mathrm{Ru}(0001),{ }^{139} \mathrm{HCl}+\mathrm{Au}(111),{ }^{133} \mathrm{CO}_{2}+\mathrm{Ni}(100),{ }^{62} \mathrm{CH}_{4}+\mathrm{Cu}(111),{ }^{63}$ and to describe vibrational energy dissipation in scattering of $\mathrm{CO}$ from $\mathrm{Au}(111),{ }^{229}$ and dissociative chemisorption of $\mathrm{CO}_{2}$ on and its recombinative desorption from $\mathrm{Pt}(111) .{ }^{462}$ Reasonably high accuracy was reported: RMSEs achieved for $\mathrm{N}_{2}+\mathrm{Ru}(0001)$ and $\mathrm{CO}_{2}+$ $\mathrm{Ni}(100)$ were $38^{139}$ and $15^{62} \mathrm{meV}$, respectively. Also, $S_{0}$ computed with the HDNN approach are in good agreement with values obtained with DFMD calculations ${ }^{62,63,133}$ (see e.g. Fig. 9a and Fig. 3 of ref. 133). The HDNN approach has been implemented ${ }^{463}$ in the LAMMPS ${ }^{464}$ MD code, and the training of the network can be parallelized. $^{465}$

Very recently, a new variety of the Behler-Parinello approach to HDNN potentials was presented by Jiang and co-workers. ${ }^{466}$ In this version of the method the atomistic neural networks are defined by descriptors consisting of $L$-dependent atomic electron densities described by Gaussian type orbitals, where $L$ is the electronic angular momentum. The method implicitly incorporates three-body terms but does not require the explicit calculation of these terms, which is thought to make the method efficient. ${ }^{466}$ The method has been applied to the interaction of $\mathrm{H}_{2}$ with $\mathrm{Cu}(111), \mathrm{Cu}(100), \mathrm{Cu}(110)$, and $\mathrm{Cu}(211)$, in a paper which also demonstrated that the same atomic networks trained on all these systems could be used to compute accurate potential energy surfaces for all four systems. ${ }^{467}$ This illustrates a fourth advantage of atomic NNPs: in principle, and if well-trained, atomic NNPs can be used to generate accurately fitted PESs for a molecule interacting with a range of different surfaces of the same metal. ${ }^{467}$ The method has also been applied to $\mathrm{H}_{2} \mathrm{O}$ interacting with $\mathrm{Pt}(110)-(1 \times 2){ }^{468}$ Atom-centered symmetry functions have been tested against three other local environment descriptors in calculations on solid state materials. ${ }^{469}$ Other machine learning methods based on atomic descriptors have also been applied to molecules interacting with metals, e.g. to $\mathrm{CH}_{4}$ interacting with $\mathrm{Pt}(111) .{ }^{470}$ Finally, symmetry adapted high-dimensional neural networks have also been used to represent electronic friction tensors of adsorbates on metals. ${ }^{471}$

\subsection{The CRP}

The CRP is an interpolation method for describing the interaction of diatomic $\mathrm{AB}$ molecules with static metal surfaces and generates PESs depending on six DOFs (six-dimensional, 6D). It was developed early on by Busnengo et al., ${ }^{472}$ and has been 

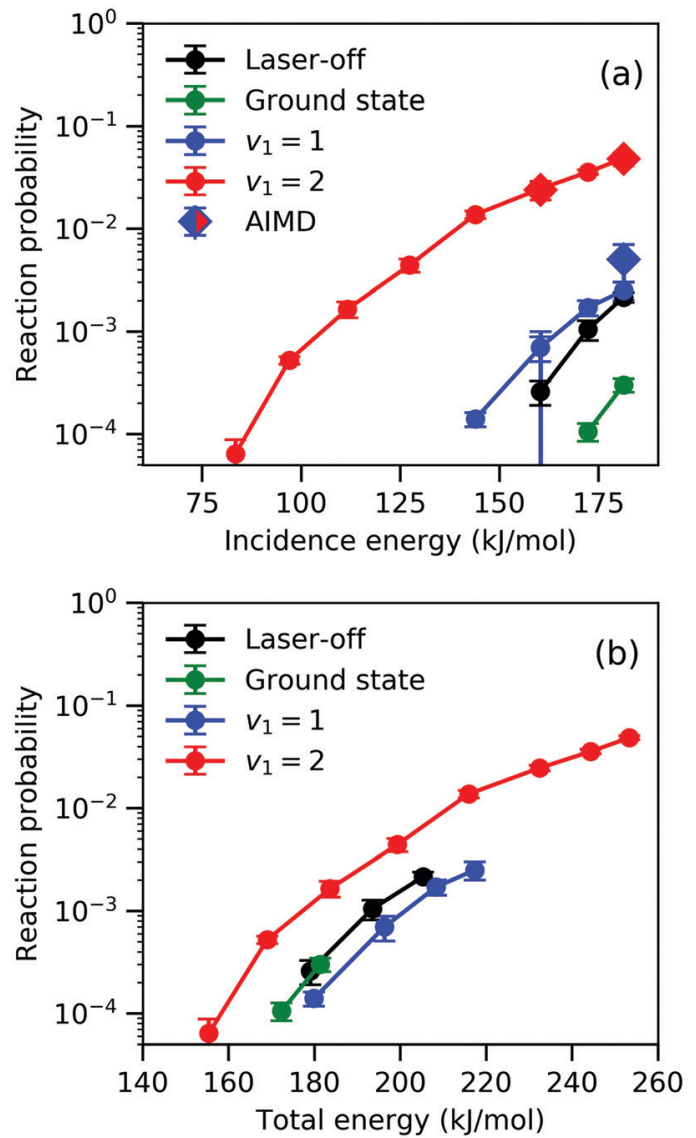

Fig. $9 S_{0}$ computed with a HD NNP (circles) and with DFMD (diamonds) on the basis of the SRP32-vdW1 DF are compared for $\mathrm{CHD}_{3}+\mathrm{Cu}(111),{ }^{63}$ for laser-off conditions, for the molecule in its ground vibrational state, and for the $\nu_{1} \mathrm{CH}$-stretch vibration excited with one quantum and with two quanta. The results are shown as a function of incidence energy (a) and of total energy (incidence + vibrational energy, panel b). Figure taken from ref. 63 (https://pubs.acs.org/doi/10.1021/acs.jpclett.9b00560). Further permission requests to be directed to the ACS.

discussed earlier in a review on $\mathrm{H}_{2}$ scattering from metal surfaces. ${ }^{28}$ We will therefore be brief in our discussion.

The idea underlying the CRP is that it is easier to interpolate a $6 \mathrm{D}$ function if it depends less strongly on the orientation and the impact site of the molecule on the surface than the actual PES. To reduce this dependence, i.e., to reduce the "corrugation", in a first step the atom-surface potentials $V_{\mathrm{A}}$ and $V_{\mathrm{B}}$ of the loose A and $\mathrm{B}$ atoms interacting with the static surface are computed and fitted, and subtracted from the $6 \mathrm{D}$ potential $V_{6 \mathrm{D}}$ describing the interaction of the $\mathrm{AB}$ molecule with respect to the surface. This leads to a $6 \mathrm{D}$ interpolation function $I_{6 \mathrm{D}}$ that is much less corrugated than the original PES $\left(V_{6 \mathrm{D}}\right)$, and $I_{6 \mathrm{D}}$ is then interpolated in a procedure more fully described in ref. 28 and ref. 472. With the proper choice of angular functions, the method is automatically permutationally invariant. In a second step, the corrugation (i.e., dependence on surface impact site) is also removed as much as possible from the potentials $V_{\mathrm{A}}$ and $V_{\mathrm{B}}$ of the loose $\mathrm{A}$ and $\mathrm{B}$ atoms interacting with the static surface. This is done by substracting from these potentials a sum of pair potentials, with the pair potential usually fitted to the interaction of the atom with the surface as it moves vertically above a surface atom (above a top site). The subtraction of the sum of pair potentials yields $3 \mathrm{D}$ interpolation functions $I_{\mathrm{A}}$ and $I_{\mathrm{B}}$, which are again easier to interpolate than the original 3D potentials $V_{\mathrm{A}}$ and $V_{\mathrm{B}}$. The CRP is similar in spirit to a procedure developed almost simultaneously by Kresse, ${ }^{473}$ who omitted the reduction of the corrugation of the atom-surface potentials by subtracting sums of pair potentials.

The accuracy of the CRP is usually tested by comparison to raw DFT data not included in the interpolation set. Usually, errors no larger than $0.4-0.7 \mathrm{kcal} \mathrm{mol}^{-1}$ are obtained in the coordinate regions relevant to reaction, so the method is quite accurate. The accuracy of the CRP has been confirmed by comparing CT results for sticking of $\mathrm{H}_{2}$ to $\mathrm{Pd}(100)$ to AIMD data for the same system. ${ }^{474}$ The CRP can be generalized to an $\mathrm{AB}$ molecule interacting with a pre-covered surface, ${ }^{118}$ and it can also be used in a context where a diatomic molecule scatters from a thermally distorted surface. ${ }^{171,475}$ Though perhaps somewhat less accurate than the PIP-NN approach for diatomic molecules interacting with static metals, the CRP is often the method of choice for obtaining global PESs for diatomic molecules interacting with static metal surfaces. ${ }^{132,156,476}$

\subsection{Other fitting methods}

Busnengo and co-workers have used a reactive force field (RFF) method with a functional form similar to one originally devised by Tersoff ${ }^{477}$ and fully described in Supporting Information (SI) to their papers, ${ }^{53,478}$ and applied it to fit PBE-DFT data for $\mathrm{CH}_{4}+$ $\mathrm{Ni}(111),{ }^{53} \mathrm{CH}_{4}+\mathrm{Pt}(111)^{53,479}$ and $\mathrm{CH}_{4}+\mathrm{Ir}(111) .{ }^{478}$ The expression they use is almost identical to the reactive bond order (REBO) expression used earlier by Busnengo and co-workers to simulate reaction of $\mathrm{H}_{2}$ on Pd surfaces. ${ }^{480}$ In the latter paper, the REBO expression used is attributed to Brenner et al., ${ }^{481}$ although this is not immediately evident from the equations presented in the papers. A very thorough assessment of the fitting errors was not yet presented for methane interacting with $\mathrm{Ni}(111)$ and $\mathrm{Pt}(111)$, but for $\mathrm{CH}_{4}+\mathrm{Pt}(111)$ the authors noted that $E_{\mathrm{b}}$ obtained with their REBO force field differed from the raw DFT values by about $1.8 \mathrm{kcal} \mathrm{mol}^{-1}$ if the surface was allowed to relax in response to the presence of $\mathrm{CH}_{4} \cdot{ }^{479}$ This suggests that chemical accuracy was not yet achieved with this type of classical force field method for $\mathrm{Ni}(111)$ and $\mathrm{Pt}(111)$. However, for $\mathrm{CH}_{4}+\operatorname{Ir}(111)$ the RMSE is below a kcal $\mathrm{mol}^{-1}$ for interaction energies up to and including the barrier height to dissociation (see Fig. S1 of ref. 478), indicating that the method is accurate enough to achieve chemical accuracy in fitting PESs.

The modified Shepard (MS) interpolation method uses energies, gradients, and Hessians computed with electronic structure theory to construct the PES as a weighted average over second-order Taylor interpolants. ${ }^{48,483}$ In investigations of reactive molecule-metal surface scattering the method was first applied to $\mathrm{H}_{2}+\mathrm{Pt}(111) .{ }^{484,485}$ The method is accurate enough to yield a good description of observables that are sensitive to an accurate representation of the PES, as shown for rotationally and diffractionally inelastic scattering of $\mathrm{H}_{2}$ from $\mathrm{Cu}(111) \cdot{ }^{117}$ In its original formulation for molecule-surface scattering ${ }^{485}$ the 
MS scheme suffered from a problem with imposing translational symmetry, ${ }^{486}$ but Frankcombe has shown that this problem can be solved by imposing plane group symmetry. ${ }^{487}$ Frankcombe has recently tested the thus modified method on a polyatomic molecule $\left(\mathrm{CH}_{4}\right)$ interacting with a model (100) surface of an fcc metal. ${ }^{488} \mathrm{He}$ has also shown that the new method can be efficiently used for molecules interacting with thermally distorted surfaces using approximate Hessians. ${ }^{489}$

The scattering of a diatomic molecule from a metal surface can also be studied with LEPS PESs. McCreery and Wolken modified an expression originally due to London, Eyring, Polanyi, and Sato to obtain a LEPS potential for a molecule scattering from a flat surface. ${ }^{490,491}$ This potential contains $D, \alpha$, and $r$ parameters modeling Morse pair potentials, and Sato parameters $\Delta$. The description of the molecule-surface interaction can be improved by making the $D, \alpha$, and $r$ parameters periodic functions of the coordinates $X$ and $Y$ describing the projection of the atoms on the surface, ${ }^{492,493}$ obtaining a periodic LEPS (PLEPS) potential. ${ }^{494} \mathrm{~A}$ shortcoming of the PLEPS expression is that the Sato parameters are taken independent of $X$ and $Y$, so that the shape and position of the barriers are restrained to be roughly the same over the surface. ${ }^{494,495}$ A more flexible periodic LEPS (called FPLEPS) potential is obtained if the Sato parameters are also made dependent on $X$ and $Y^{494,496}$ An advantage of the FPLEPS method is that a fit of a PES for a diatomic molecule interacting with a static surface can be obtained with an order of magnitude less points (say 500) than needed for an accurate fit with the CRP. ${ }^{494}$ However, the FPLEPS expression is also less accurate, as was demonstrated for $\mathrm{N}_{2}+\mathrm{W}(100)$ where the method could not achieve chemical accuracy for the early barriers occurring far away from the surface (errors $\sim 100 \mathrm{meV}$ ), and in a few cases failed miserably for late barriers (errors $\sim 250 \mathrm{meV}$ ). Recently the method has been used to fit a PES for, for example, $\mathrm{O}_{2}+$ $\mathrm{Cu}(100)^{497}$ and $\mathrm{O}_{2}+\mathrm{Al}(111) \cdot{ }^{35}$ In the latter case, the advantage that fewer points could be used to fit the PES came in handy, as an expensive electronic structure method (the ECW method) was used to compute electronic energies. ${ }^{35}$ However, the limited accuracy of the FPLEPS method also led to some of the uncertainties contained in the conclusions of this important work. ${ }^{35}$

As already noted, PIPs can be used in a NN approach to PES fitting. PIPS have also been used briefly to fit molecule-surface PESs in an approach not using NNs. ${ }^{498}$ In this approach, ${ }^{498}$ a pseudo "surface atom" is introduced at a specific impact site, which is usually chosen to correspond with the TS of the system. The PES is then expanded in polynomials of Morse-like functions of the interatomic distances between the "atoms" of the system, i.e., all the atoms in the molecule and the pseudo-atom. The fitting expression is made permutationally invariant in the atoms of the molecule belonging to the same chemical elements, and least-squares fitting of the coefficients is performed. In this approach, the flat surface approximation (FSA) is invoked, i.e., the approximation is made that the PES does not depend on the coordinates for translational motion parallel to the surface, and on the azimuthal rotation of the molecule about the surface normal. ${ }^{498}$ The method has been applied to $\mathrm{H}_{2} \mathrm{O}+\mathrm{Cu}(111),{ }^{108,119,499} \mathrm{D}_{2} \mathrm{O}+\mathrm{Ni}(111),{ }^{78}$ and $\mathrm{CH}_{4}+\mathrm{Ni}(111),{ }^{60,500}$ and has been reviewed in ref. 498 .
It is also possible to combine different methods. For instance, it is possible to use the CRP, but to fit the $6 \mathrm{D}$ interpolation function $I_{6 \mathrm{D}}$ with the NN approach, as has been done for a few systems. ${ }^{452,501}$

In calculations with the multi-configuration time-dependent Hartree (MCTDH) method, it is computationally favorable to use a PES that is in a sum of products form. ${ }^{502}$ Ideally, products of $1 \mathrm{D}$ functions of the DOFs used in the MCTDH calculations are used. The POTFIT method ${ }^{502-504}$ fits potentials to this form, and has been used to describe for instance $\mathrm{H}_{2}+\mathrm{Pt}(111)^{505}$ and $\mathrm{CH}_{4}+\mathrm{Pt}(111)^{87}$ (the latter in a reduced dimensionality framework). A new method called canonical polyadic decomposition also allows to bring the PES in a sum of products form, and thanks to some of its features it can be used efficiently for highdimensional systems. ${ }^{506}$ As already mentioned in Section 3.1 there are also NN methods ${ }^{458-460}$ that result in PESs with a sum of products form.

\section{Dynamical models and methods and computation of observables}

Once an electronic structure model has been set up for the system (whether implemented through a PES or direct dynamics), a dynamical model and method are required to compute $S_{0}$ (or rate constants if kinetics results are required). The model used determines whether (i) only motion in the molecular DOFs is modeled (BOSS model) and which molecular DOFs are modeled explicitly, or whether (ii) also surface atom motion is modeled (BO moving surface or BOMS model), or whether instead (iii) electronically non-adiabatic effects like ehp excitation are modeled (non-BOSS or NBOSS model), or whether (iv) both nonadiabatic effects and surface phonons are modeled (NBOMS). In discussing these models and their use, we will closely follow a recent review paper by Reuter and co-workers. ${ }^{266}$ It is good to start with a caveat: As these authors note, still much is unclear about the relative importance of the two energy dissipation channels (ehp and surface phonon excitation) for moleculemetal surface reactions. ${ }^{266}$ The topic is important not only because these channels may require accurate modeling to extract accurate reaction barriers from dynamics simulations of MB sticking experiments. ${ }^{10}$ As Reuter and coworkers note, the energy released in for instance DC reactions may well affect how heterogeneously catalyzed processes proceed, which is usually neglected in current microkinetic modeling of these important processes. $^{266}$

The dynamical method determines how the equations of motion are solved, possibly in connection with how the electronic structure calculations are implemented. Finally, a dynamics calculation should result in observables, and we will also discuss how these are computed.

\subsection{Dynamical models}

4.1.1. The BOSS model. Many molecule-metal surface reactions can already be described with quantitative or semiquantitative accuracy while neglecting electronically non-adiabatic effects and treating the metal surface as static, where the atoms 
occupy their ideal metal lattice positions. This is particularly true for activated dissociation of $\mathrm{H}_{2}$ on cold $\left(T_{\mathrm{s}} \leq 300 \mathrm{~K}\right)$ metal surfaces, and is illustrated by the achievement of chemically accurate results with the BOSS model for sticking of $\mathrm{H}_{2}$ on $\mathrm{Cu}(111),{ }^{43} \mathrm{Cu}(100),{ }^{46}$ and Pt(111), ${ }^{156}$ with the use of SRP-DFs. In attempts to develop SRP-DFs highly accurate descriptions were likewise achieved with the BOSS model for $\mathrm{H}_{2}+\mathrm{Ru}(0001),{ }^{151} \mathrm{Ni}(111),{ }^{476} \mathrm{Ag}(111)^{152}$ and $\operatorname{Pt}(211){ }^{48}$

The available evidence also suggests that, in the absence of SRP or candidate SRP DFs (c-SRP DFs), activated dissociation of $\mathrm{H}_{2}$ on metals can be modeled with at least semi-quantitative accuracy with the BOSS model, ${ }^{80,111,116,186,507-510}$ with deviations from experiment usually stemming from the inaccuracy of the DF employed. BOSS calculations on $\mathrm{H}_{2}$ scattering from metals have also been used successfully to reproduce and explain mechanistic trends, ${ }^{169,511}$ or to predict trends ${ }^{187}$ later confirmed in experiments. $^{23,166}$ Finally, BOSS calculations on $\mathrm{H}_{2}$-metal surface scattering have also been performed to test whether specific dynamical approximations may lead to accurate results. For instance, several authors have found that full-dimensional (6D) calculations on DC are accurately reproduced by an approximation using site-averaging of explicit dynamics (SAED) results of 4D calculations modeling only motion in the vibrational and rotational coordinates of $\mathrm{H}_{2}$, and in its motion towards the surface. ${ }^{76,102}$ However, we note that calculations on scattering of $\mathrm{H}_{2}$ from metal surfaces with the BOSS model may also fail rather badly for specific observables in activated systems, as found for vibrationally inelastic scattering of $\mathrm{H}_{2}$ from $\mathrm{Cu}(111) .{ }^{177}$ For more details on modeling of $\mathrm{H}_{2}$ reacting on metals, see a recent review paper. $^{28}$

The BOSS model has also been used to study reactive scattering of heavier diatomic molecules from metal surfaces. For a few of these systems (i.e., $\mathrm{HCl}+\mathrm{Au}(111)$ and $\mathrm{Ag}(111))$ the SAED approximation is accurate. ${ }^{106,457}$ In many cases using the BOSS model leads to semi-quantitative agreement with experiment, as found for e.g. $\mathrm{O}_{2}+\mathrm{Ag}(111)^{82}$ and $\mathrm{Al}(111),{ }^{35} \mathrm{~N}_{2}+\mathrm{W}(110)^{512}$ and $\mathrm{Ru}(0001) \cdot{ }^{513}$ For $\mathrm{HCl}+\mathrm{Au}(111)$ BOSS dynamics calculations are in disagreement with experiments on sticking, ${ }^{134}$ but it is likely that this is mostly due to the DF used.

The BOSS model has also been used to study reaction of polyatomic molecules on metal surfaces. It has been successful at describing the reaction of $\mathrm{H}_{2} \mathrm{O},{ }^{131} \mathrm{NH}_{3}{ }^{66}$ and $\mathrm{CH}_{4}$ with metal surfaces with semi-quantitative accuracy for several systems. The BOSS method has also been used to predict trends in reactivity of such systems, for instance regarding the effect of pre-exciting different vibrations, ${ }^{66,67,108,144}$ different rotational states, ${ }^{108,131}$ of the incidence angle $\theta_{i},{ }^{121}$ of rotational dynamics, ${ }^{87}$ and of steric effects. $^{131,137}$ Several studies have focused on the validity of making reduced dimensionality approximations, ${ }^{100,123}$ and in many cases site-averaging approximations were found to perform well (e.g. for $\left.\mathrm{H}_{2} \mathrm{O}+\mathrm{Ni}(100),{ }^{98} \mathrm{H}_{2} \mathrm{O}+\mathrm{Cu}(100),{ }^{61} \mathrm{CH}_{4}+\mathrm{Ni}(111)^{145}\right)$.

While the BOSS model has therefore also been used to study reactive scattering of molecules heavier than $\mathrm{H}_{2}$ from metals, we note that dynamics studies using this model have so far not yet reached a chemically accurate description of these systems. The reasons for this will be discussed in the subsequent sections and concern the role of phonons and ehp excitation.
Furthermore, also in studies using the BOSS model, for achieving quantitative accuracy it is essential to include all or most molecular DOFs. ${ }^{61,513,514}$ As noted by Reuter and co-workers, ${ }^{266}$ the absence of dissipation in the model means that an ad hoc criterion needs to be used whether sticking has occurred or not (typically this is decided on the basis of a dissociating bond achieving a critical value). Finally, as also noted by them, ${ }^{266}$ the BOSS model implies an efficient two-step procedure to compute $S_{0}$ : first a PES is computed, and then the dynamics is done.

4.1.2. The BOMS model. Quite a few implementations exist of the BOMS model that can usefully be applied in the framework of attempts to obtain a chemical accurate description of sticking of molecules to metal surfaces. As will become clear below, these implementations differ in which couplings of phonon and projectile motion are taken into account (e.g. electronic and mechanical, see below), and whether instantaneous couplings between these motions are modeled. They also differ in the extent to which motion in one, three, or many extra surface atom DOFs needs to be modeled in the dynamics, which is relevant to the computational expense. Some implementations are applied " $a$ posteriori", i.e., after the dynamics has been performed for a static surface, making these implementations inexpensive to apply. Implementations also differ in whether and to what extent they can describe phonon energy dissipation away from the reaction zone, and in whether and to what extent they can take into account the phonon fine structure of the metal surface. Finally, implementations differ in their computational expense. In discussing the implementations below, we attempt to address all of these issues.

One of the simplest models for describing the effect of surface atom motion is the so-called surface oscillator (SO) model. ${ }^{515,516}$ Assumptions underlying this model are that in the TS the molecule dissociates above a top layer surface atom (a "top site"), and that the interaction of the molecule with the surface "moves" with this surface atom, i.e., the interaction depends on the distance of the molecule to this atom instead of the distance to the surface. The vibrational motion of the surface is approximately represented by the motion of this one independently vibrating surface atom (the Einstein model), which may be taken in either one dimension (perpendicular to the surface) or in three dimensions. The oscillating atom is usually treated as a harmonic oscillator, but it can also be treated as a Morse oscillator, thereby introducing anharmonicity. ${ }^{517}$ The barrier location moves with the vibrating atom, but not its height. As shown early on by Busnengo and co-workers for $\mathrm{H}_{2}$ reacting on Pd surfaces, ${ }^{475,518}$ using the SO model to treat surface motion may help with describing the effect of transient trapping of the molecule on the $S_{0}$, both in its dependence on $E_{i}$ and $T_{\mathrm{S}}$. Holloway and co-workers showed that the SO-model helped explain the Arrhenius like form of the $T_{\mathrm{s}}$-dependence of inelastic scattering probabilities for the activated dissociation system $\mathrm{H}_{2}+\mathrm{Cu}(111) .{ }^{519}$

The generalized Langevin oscillator (GLO) method ${ }^{475,520,521}$ extends the SO model by introducing energy dissipation and (if $T_{\mathrm{s}}$ is taken greater than $0 \mathrm{~K}$ ) thermal fluctuations, thereby also modeling the surface as a bulk thermal bath. The GLO method does this by coupling the motion of the oscillating surface 
atom to a "ghost oscillator", which is subject to "phononic friction", while thermal fluctuations can be imposed in a way that ensures that the fluctuation-dissipation theorem is obeyed. The GLO method has been applied to e.g. sticking of $\mathrm{H}_{2}$ to Pd surfaces, ${ }^{475}$ vibrational excitation of $\mathrm{H}_{2}$ scattering from $\mathrm{Cu}(111),{ }^{168}$ sticking of $\mathrm{N}_{2}$ to $\mathrm{W}$ surfaces, ${ }^{86,132}$ sticking of $\mathrm{O}_{2}$ to $\mathrm{Pd}(100)^{522}$ and $\mathrm{Cu} / \mathrm{Ru}(0001){ }^{130}$ and scattering of $\mathrm{O}_{2}$ from $\mathrm{Ag}(111)^{94}$ and $\mathrm{Pt}(111){ }^{523}$

A more sophisticated treatment than the GLO is due to Jackson and co-workers, ${ }^{524,525}$ and has been called various names, including e.g. the lattice reconstruction sudden (LRS) model (the term we will use) and the lattice sudden model. This model is not only a refinement of the SO-model, it also is an important extension, recognizing that for systems like $\mathrm{CH}_{4}$ interacting with a metal surface $E_{\mathrm{b}}$ (and not just its location) may very strongly with the displacement of the surface atom below the dissociating molecule, ${ }^{50,524,525}$ as first noted for $\mathrm{CH}_{4}+\operatorname{Ir}(111)$ by Jónsson and coworkers. $^{526}$ The refinement consists in recognizing that the dependence of the barrier location $Z_{\mathrm{b}}$ on the surface atom displacement coordinate $Q$ can to a good approximation be written as

$$
Z_{\mathrm{b}}(Q)=Z_{\mathrm{b}}(0)+\alpha Q
$$

where $\alpha$ is called the mechanical coupling (parameter), and is taken as 1.0 in the SO method. The extension consists in taking into account the dependence of $E_{\mathrm{b}}$ on $Q$ through

$$
E_{\mathrm{b}}(Q)=E_{\mathrm{b}}(0)-\beta Q
$$

where $\beta$ is called the electronic coupling (parameter), and is usually negative. The electronic coupling is taken into account ${ }^{30}$ by first computing an "intermediate" $T_{\mathrm{s}}$-dependent $S_{0}$ as

$$
S_{0}^{Q \mathrm{av}}\left(E_{i}, T_{\mathrm{s}}\right)=\int P_{\text {lat }}\left(Q ; T_{\mathrm{s}}\right) S_{0}\left(E_{i} ; Q\right) \mathrm{d} Q,
$$

with the additional approximation ${ }^{30}$ that the $S_{0}$ computed for a static lattice with surface atom displacement $Q$ is taken as

$$
S_{0}\left(E_{i} ; Q\right) \approx S_{0}\left(E_{i}+\beta Q ; Q=0\right) .
$$

In eqn (7) $P_{\text {lat }}\left(Q ; T_{\mathrm{s}}\right)$ is the probability of finding a surface atom displacement $Q$ at the surface temperature $T_{\mathrm{s}}$. The use of eqn (8) implies that for positive $Q$ the sticking probability computed for $Q=$ 0 is simply shifted towards lower $E_{i}$ with a "shifting energy" $\Delta E_{i}(Q)=$ $\beta Q$, thereby enhancing reaction. The mechanical coupling is taken into account by modeling the surface atom through the surface mass (SM) model. ${ }^{516}$ In this model, the surface atoms' vibrations are taken into account by assuming that the molecule collides with a moving mass that obeys a momentum distribution as if the surface atom it collides with were vibrating, so that the reaction probability becomes dependent on the relative velocity of the molecule with respect to the moving surface atom. This implies that the final $T_{\mathrm{s}}$-dependent $S_{0}$ is computed as

$$
\begin{aligned}
S_{0}\left(E_{i}, T_{\mathrm{s}}\right)= & \int \mathrm{d} E_{\mathrm{CM}} \sqrt{\frac{M_{\mathrm{s}}^{\prime}}{4 \pi k T \mu_{\mathrm{T}} E_{\mathrm{CM}}}} \exp \left[-\frac{M_{\mathrm{s}}^{\prime}}{2 k T}\left(\sqrt{\frac{2 E_{\mathrm{CM}}}{\mu_{\mathrm{T}}}}\right.\right. \\
& \left.-\left(\sqrt{\frac{2 E_{i}}{M}}\right)^{2}\right] S_{0}^{Q \mathrm{av}}\left(E_{\mathrm{CM}}\right)
\end{aligned}
$$

where the relative translational energy is given by

$$
E_{\mathrm{CM}}=\frac{1}{2} \mu_{\mathrm{T}}\left(\sqrt{\frac{2 E_{i}}{M}}-\alpha \frac{P_{0}}{M_{\mathrm{s}}}\right)^{2} .
$$

In eqn (9), $M_{\mathrm{s}}^{\prime}=M_{\mathrm{s}} / \alpha^{2}, M_{\mathrm{s}}$ is the mass of the surface atom, and the reduced mass $\mu_{\mathrm{T}}=M_{\mathrm{s}}^{\prime} M /\left(M_{\mathrm{s}}^{\prime}+M\right)$, with $M$ equal to the mass of the impinging molecule. While Jackson and co-workers originally used an Einstein model to describe the coordinate and momentum distribution of the surface atom, more recently they have turned to using the Debye model (which describes a single atom vibrating in the field of other vibrating surface atoms). ${ }^{527}$

Zhang and co-workers ${ }^{73,528}$ have proposed changes to the electronic coupling model as provided by eqn (6). In their VTSR model, ${ }^{528}$ the shifting energy $\left(V\right.$, which is $\Delta E_{i}(Q)$ in our notation above) is computed based on the transition state (TS) for the relaxed surface $(R) .{ }^{529}$ That is, $E_{\mathrm{b}}$ is allowed to depend nonlinearly on $Q$, and a relaxation in $Q$ is carried out to determine the variation of $E_{\mathrm{b}}$ with $Q$, leading to $\Delta E_{i}(Q)=E_{\mathrm{b}}(Q=0)-$ $E_{\mathrm{b}}(Q) .{ }^{528}$ This gave improved agreement with phonon-sudden calculations in which 7D $S_{0}$ were explicitly computed with a different DFT-PES for different $Q$ with subsequent averaging. ${ }^{528}$ While they argue that their model represents an improved model over eqn (6), is not clear whether the VTSR model is a better a posteriori model than the LRS model: The VTSR model does not take mechanical coupling into account. ${ }^{529}$ The "P-averaged" (i.e., mechanically coupled) energy-shifted (according to eqn (6)) results of Jackson and co-workers were in better agreement with calculations including explicit coupling with phonons than their " $P$-averaged" sudden results. ${ }^{524}$ Therefore, reproducing sudden results (as done by Zhang and co-workers ${ }^{528}$ ) may not be a guarantee for obtaining a better a posteriori model to describe phonon effects than the LRS model.

If the motion of the molecule is electronically coupled to more than one surface atom, this can also be taken into account with the LRS model. ${ }^{78}$ Finally, taking into account the mechanical coupling implies that the " $Q=0$ " problem is solved while replacing $\mathbf{M}$ by $\mu_{\mathrm{T}}$, next implementing the sudden approximation to apply the electronic coupling (effectively averaging over $Q$ ), and then completing the implementation of mechanical coupling by averaging over the momentum distribution for relative motion. This is usually done intertwined with averaging over the molecule's impact sites, as fully described in the ESI to ref. 78.

Fig. 10 shows how the mechanical coupling and the electronic coupling impact the $T_{\mathrm{s}}$-dependent reaction probabilities with the approximations made for $\mathrm{CH}_{4}+\mathrm{Ni}(111)$, with the parameters taken as appropriate for this system. ${ }^{81}$ It is immediately obvious that, for the $T_{\mathrm{s}}$ addressed (475 K), at low $E_{i}$ the electronic coupling $(\beta=1.16, \alpha=0)$ affects the sticking much more than the mechanical coupling $(\alpha=0.7, \beta=0)$. Furthermore, turning on the electronic coupling has the effect of increasing $S_{0}\left(E_{i}, T_{\mathrm{s}}\right)$ for all $E_{i}$, whereas turning on the mechanical coupling increases $S_{0}\left(E_{i}, T_{\mathrm{s}}\right)$ at low $E_{i}$ but decreases it at high $E_{i}$ compared to the rigid surface case. The two effects reinforce each other at low $E_{i}$ where they both increase the reactivity. Using the actual value of $\alpha$ rather than the simple SM model $(\alpha=1)$ leads to distinct results 


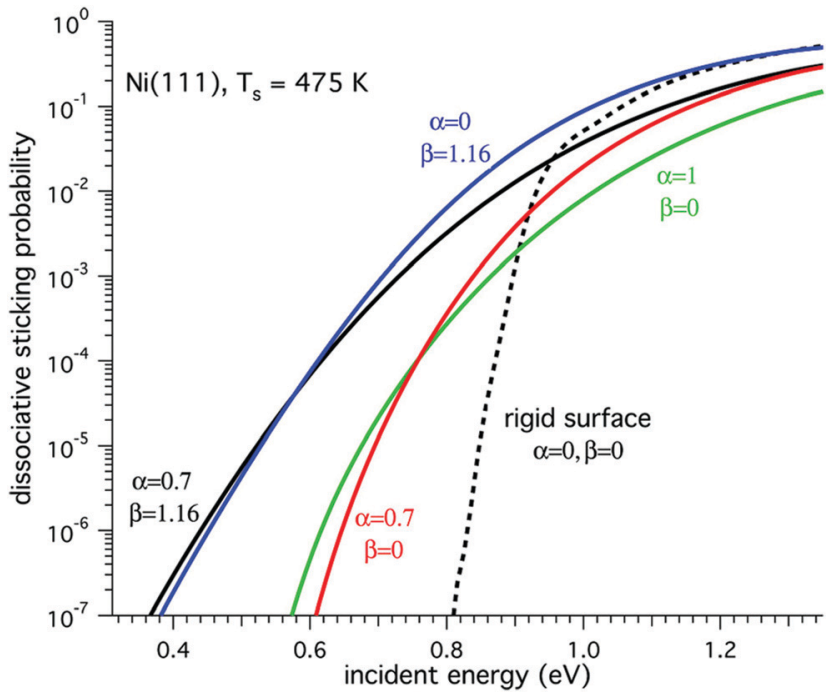

Fig. $10 \mathrm{~S}_{0}$ computed $^{81}$ for $\mathrm{CH}_{4}+\mathrm{Ni}(111)$ with the $\mathrm{RPH}$ method using the LRS model for phonon coupling are shown as a function of $E_{i}$ for $T_{\mathrm{S}}=475 \mathrm{~K}$, for the four combinations of the electronic and mechanical coupling parameters indicated. Results obtained with the static surface approximation are presented for comparison. Reprinted from [The dissociative chemisorption of methane on Ni(111): The effects of molecular vibration and lattice motion. J. Chem. Phys., 2013, 138, 174705], with the permission of AIP Publishing.

but the effect is not very large. It is clear from Fig. 10 that it is not a good idea to ignore the electronic coupling if $E_{\mathrm{b}}$ shows a strong dependence on surface atom displacements, as often found for polyatomic molecules like $\mathrm{CH}_{4}$ and $\mathrm{H}_{2} \mathrm{O}$ interacting with TM surfaces.

The LRS model of Jackson and coworkers has been used extensively in QD calculations with the RPH method mo,203 $^{5}$ on $\mathrm{CH}_{4}$ reacting with $\mathrm{Ni}(100),{ }^{50,89} \mathrm{Ni}(111),{ }^{21,30,81} \mathrm{Ni}(211),{ }^{179}$ $\operatorname{Pt}(111),{ }^{30,527}$ and with $\mathrm{Pt}(211),{ }^{162}$ on $\mathrm{H}_{2} \mathrm{O}$ reacting with $\mathrm{Ni}(111),{ }^{72,77} \mathrm{Ni}(110),{ }^{72}$ and $\mathrm{Ni}(100),{ }^{72}$ and on $\mathrm{CO}_{2}$ reacting on $\mathrm{Ni}(100) .{ }^{71}$ It has also been used in conjunction with reduced dimensional quantum wave packet calculations on dissociation of $\mathrm{CH}_{4}$ on Ni surfaces ${ }^{73,528}$ and of $\mathrm{H}_{2} \mathrm{O}$ on Ni(111), ${ }^{155}$ and with QCT calculations on $\mathrm{CH}_{4}+\mathrm{Ni}(111) .{ }^{161,167}$ Calculations with the LRS model have been in good agreement with the measured $T_{\mathrm{s}}$-dependence of the sticking of $\mathrm{CH}_{4}$ on $\mathrm{Pt}(111)^{30}$ and on $\mathrm{Ni}(111) ;{ }^{30}$ for the latter system good agreement with the experimental trend was found for $T_{\mathrm{s}}$ ranging from 90 to $475 \mathrm{~K}$ (see also Fig. 11). ${ }^{21}$ Likewise, applying the LRS model to results of QCT calculations performed for a fixed lattice gave excellent agreement with AIMD calculations for the mobile surface for $\mathrm{CH}_{4}+\mathrm{Ni}(111)$, although these calculations did raise the question why the LRS model performed better with the use of the Einstein than with the Debye model. ${ }^{167}$ Jackson's LRS model can also be used in conjunction with semi-classical calculations. ${ }^{68}$

It is also possible to compute $S_{0}$ based on a quantum sudden approach (QD with the phonon sudden approximation, PSA). In this case, initial internal state (i) selected reaction probabilities for the SO in the initial phonon state $n$ (we are assuming

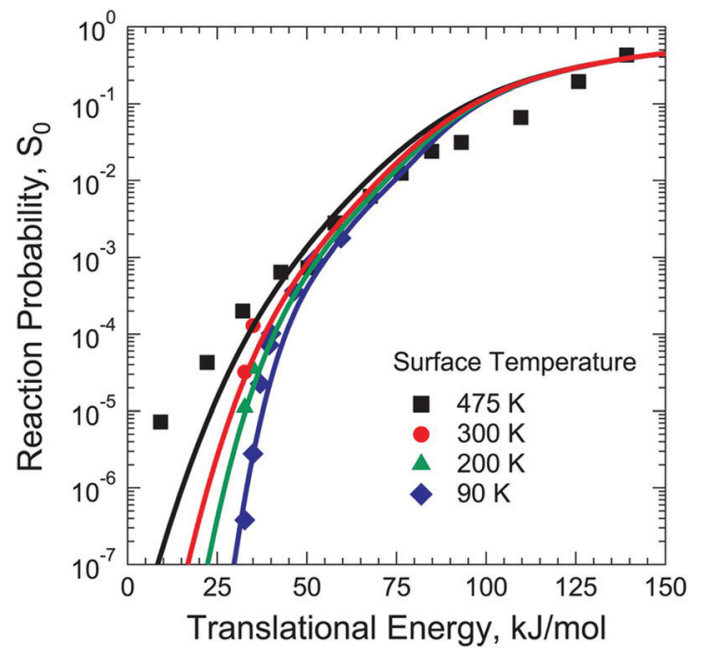

Fig. 11 Initial-state resolved $S_{0}$ computed ${ }^{21}$ with the RPH method using the LRS model for phonon coupling are shown for $\mathrm{CH}_{4}\left(\nu_{3}, v=1\right)+\mathrm{Ni}(111)$ as a function of $E_{i}$ for the values of $T_{s}$ indicated. Reprinted with permission from (V. L. Campbell, N. Chen, H. Guo, B. Jackson and A. L. Utz, Substrate vibrations as promoters of chemical reactivity on metal surfaces. J. Phys. Chem. A, 2015, 119, 12434-12441). Copyright (2015) American Chemical Society.

the Einstein model is used) can be computed for fixed $E_{i}$ according to ${ }^{171}$

$$
R_{i n}\left(E_{i}\right)=\int \mathrm{d} Q\left[1-\sum_{\boldsymbol{f} n^{\prime}}\left|S_{\boldsymbol{i n}, \boldsymbol{f} n^{\prime}}\left(E_{i} ; Q\right)\right|^{2}\right]\left|\chi_{n}(Q)\right|^{2} .
$$

The $S$-matrix elements for the transition from the $(i n)$ state to the $\left(f n^{\prime}\right)$ state, where $i(f)$ is the initial rovibrational (final rovibration-diffraction) state of the molecule and $n^{\prime}$ the final phonon state, can be computed from QD calculations for fixed $Q$, and then averaging over fixed $Q$ dynamics results. ${ }^{171} \mathrm{With}$ the averaging over $Q$ (see also eqn (11)), the electronic coupling (usually the most important) is taken into account. The method has been tested by comparing phonon-sudden $(6+1)$ QD with full-dimensional (7D) QD calculations for $\mathrm{H}_{2}+\mathrm{Cu}(111)$, showing good agreement for low $E_{i}$ and excellent agreement for high $E_{i}$, for initial-phonon-state selected reaction probabilities. ${ }^{171}$ The $T_{\mathrm{s}}$-dependence of $S_{0}$ can be incorporated by performing a "surface thermal average" of the $R_{i n}(E)$, and subsequently averaging over the distributions of $E_{i}$ and of the internal states of the molecules in the MB. Compared to the LRS and VTSR models, in taking into account the electronic coupling the variation of the whole potential with $Q$ is taken into account, and not just the variation of $E_{\mathrm{b}}$ with $Q$. However, the PSA does not take mechanical coupling into account. For $\mathrm{H}_{2}$ metal surface scattering, in principle it is possible to study the full effect of phonon motion by simply adding surface DOFs, but in practice this has so far only been done by adding one surface DOF, in 7D QD calculations on $\mathrm{H}_{2}+\mathrm{Cu}(111) .{ }^{171}$

Of the model implementations discussed above, none contains explicit electronic coupling between the molecule and the surface. More specifically, the SO cannot respond instantaneously to the electronic coupling enacted by the incoming molecule, although in 
the SO and GLO methods it can respond to the instantaneous mechanical coupling. Zhou and Jiang ${ }^{153}$ have recently come up with a modified GLO (MGLO) approach that solves this problem. Instead of simply replacing the molecule surface interaction $V(\boldsymbol{R})$ by $V(\boldsymbol{R}-\boldsymbol{Q})$ (with $\boldsymbol{R}$ denoting the coordinates of the atoms in the molecule, and $\boldsymbol{Q}$ those of the SO), $V(\boldsymbol{R})$ is replaced by $V(\boldsymbol{R}-\boldsymbol{Q})+$ $V_{\mathrm{c}}\left(Z_{\mathrm{CM}}, Q\right)$, where $Z_{\mathrm{CM}}$ is the distance of the molecule's centre-ofmass to the surface. Following earlier work of Saalfrank and co-workers, ${ }^{530}$ the electronic coupling potential $V_{\mathrm{c}}\left(Z_{\mathrm{CM}}, Q\right)$ is taken as a linear coupling (as also done in the LRS model), which however vanishes at large molecule-surface distances. The possibility that the mechanical coupling parameter $\alpha$ differs from 1 is additionally taken into account by scaling the mass of the surface and ghost oscillators in the MGLO approach accordingly. ${ }^{153} S_{0}$ computed using the MGLO approach for $\mathrm{CHD}_{3}+\mathrm{Ni}(111)$ were in remarkably good agreement ${ }^{153}$ with DFMD calculations on the same system. ${ }^{44}$ While further testing of the approach may be required, this approach looks very promising for systems where the electronic and mechanical coupling between molecule and lattice is mainly due to the interaction of the molecule with a single surface atom, as appears to be the case for many systems in which $\mathrm{CH}_{4}$ interacts with a transition metal surface.

None of the methods discussed above really describes the effects of the collective motion beyond perhaps taking into account that the atoms' vibrational amplitudes are taken from the Debye model rather than the Einstein model. We now discuss methods that do take additional effects of collective surface phonon motion into account.

Perhaps the simplest of these methods is the static corrugation model (SCM). ${ }^{531}$ In this model, the surface atoms are not allowed to move during the dynamics calculation, but all atoms are displaced from their ideal lattice positions with amplitudes taken from the Debye model. The method describes electronic coupling, but not mechanical coupling. The molecule-distorted surface interaction energies are based on DFT calculations, using fits to describe the coupling between the molecular and the phonon motion. ${ }^{160,531}$ Calculations on $\mathrm{H}_{2}+\mathrm{Cu}(111)$ established that the model yields good agreement with initial-state selected reaction probabilities up to $T_{\mathrm{s}}=925 \mathrm{~K}$ if in addition to the static surface atom displacements also the thermal expansion of the $\mathrm{Cu}$ lattice was taken into account. ${ }^{160,531}$ The comparison with subsequent QCT calculations using a $0 \mathrm{~K} \mathrm{H}_{2}+\mathrm{Cu}(111)$ PES and a $925 \mathrm{~K}$ expanded lattice PES and AIMD calculations suggested that the following $T_{\mathrm{s}}$-dependent factors affect the reaction of $\mathrm{H}_{2}$ on $\mathrm{Cu}(111)$ through collective surface atom motion: surface expansion (especially regarding the distance between the top two layers of the surface) leads to enhanced reaction for all $E_{i}$, while static surface atom displacements and allowing motion in the surface during the dynamics taken together shift the reaction probability curve to higher $E_{i}$, and broaden this curve. ${ }^{172}$

The DFMD ${ }^{532}$ implementation of the BOMS model is in principle able to model all effects of (collective) surface phonon motion and of $T_{\mathrm{s}}$ on $S_{0}$. The method has been applied to achieve chemical accuracy for sticking of $\mathrm{CHD}_{3}$ to $\mathrm{Ni}(111),{ }^{44} \mathrm{Pt}(111),{ }^{47}$ and $\operatorname{Pt}(211),{ }^{47,84}$ with $T_{\mathrm{s}}$ approximately equal to $500 \mathrm{~K}$. More broadly,
DFMD has been used to study the following systems in which a polyatomic molecule reacts with or is formed at a metal surface: sticking of $\mathrm{CHD}_{3}$ on $\mathrm{Ni}(111),{ }^{44} \mathrm{Pt}(111),{ }^{47,51,84,157,164} \mathrm{Pt}(110)$ $(2 \times 1),{ }^{182} \operatorname{Pt}(211),{ }^{47,164,165} \operatorname{Pt}(210),{ }^{181} \operatorname{Pd}(111),{ }^{53} \mathrm{Cu}(111),{ }^{63}$ $\mathrm{Cu}(211),{ }^{185}$ and single atom surface alloys of $\mathrm{Cu}(111),{ }^{185}$ of $\mathrm{CH}_{4}$ on $\operatorname{Ir}(332),{ }^{534}$ of $\mathrm{CH}_{3} \mathrm{OH}$ on $\mathrm{Cu}(111),{ }^{183}$ of water on $\mathrm{Ni}(111),{ }^{184}$ of $\mathrm{NH}_{3}$ on $\mathrm{Ru}(0001),{ }^{535}$ of $\mathrm{CO}_{2}$ on $\mathrm{Ni}(100),{ }^{62,69,70} \mathrm{CO}_{2}$ formation through recombinative desorption from $\mathrm{Pt}(111)^{462,534}$ and $\mathrm{Pt}(332),{ }^{534}$ and $\mathrm{CO}_{2}$ formation through oxidation of surface adsorbed $\mathrm{CO}$ by impinging O-atoms on $\mathrm{Pt}(111) .{ }^{536}$ DFMD has also been used to study systems in which a diatomic molecule reacts with a metal surface. Topics addressed have been sticking of $\mathrm{H}_{2}$ on $\mathrm{Cu}(111),{ }^{170,175}$ $\mathrm{Cu}(100),{ }^{173} \mathrm{Cu}(211),{ }^{511}$ Pd single atom surface alloys of $\mathrm{Cu}(111),{ }^{83}$ $\operatorname{Pd}(100),{ }^{474,537}$ H-precovered Pd surfaces, ${ }^{532,538,539}$ sulfur-precovered $\operatorname{Pd}(100),{ }^{539}$ and CO-precovered $\mathrm{Ru}(0001),{ }^{188}$ sticking of $\mathrm{N}_{2}$ on $\mathrm{W}(110),{ }^{86,88,101,149}$ sticking of $\mathrm{O}_{2}$ on $\mathrm{Pt}(111),{ }^{93}$ and sticking of $\mathrm{HCl}$ on $\mathrm{Au}(111){ }^{85,136}$

In principle, reflection of dissipated energy from the bottom of the metal slab modeling the surface may be problematic for non-direct dissociation in a DFMD simulation. If this is suspected, an Andersen thermostat ${ }^{540}$ or a Nosé-Hoover thermostat ${ }^{541}$ can be coupled to the atoms in the lower layer of the metal slab to allow for energy dissipation while maintaining the modeled $T_{\mathrm{s}}$ in the long term. ${ }^{93}$

Of course, with DFMD nuclear motion is treated classically. The validity of a classical approach for the dynamics of a molecule or atom scattering from a surface can be assessed by computing the argument of the Debye-Waller (DW) factor ${ }^{542}$

$$
2 W=\frac{3 p^{2} T_{\mathrm{s}}}{M_{\mathrm{s}} k \Theta_{\mathrm{D}}^{2}}
$$

Experience ${ }^{543,544}$ suggests that the surface atoms can be treated classically it $T>\Theta_{\mathrm{D}}$ (the surface Debye temperature), and if $2 W>6$ (in eqn (12)), $p^{2}$ is the average of the square of the change in momentum of the scattering molecule.

Although the DFMD (or AIMD) method in principle contains all ingredients needed to model molecule-metal surface scattering under conditions where classical mechanics is applicable, it is still expensive to apply, with the number of DFMD trajectories to model reaction at a specific experimental condition usually restricted to $\approx 1000$. At present, in practice this precludes calculations of statistically accurate $S_{0}<0.01$, and on systems in which the sticking is considerably affected by long-time events like trapping. It is therefore fortunate that reactive molecule-metal surface scattering can now also be modeled ${ }^{139}$ with the Behler-Parinello $\operatorname{method}^{441}$ and variants of this method ${ }^{466}$ for constructing high-dimensional neural network potentials (HDNNPs, see also Section 3.1). In this framework the QCT method has now been applied successfully to sticking of $\mathrm{N}_{2}$ to $\mathrm{Ru}(0001),{ }^{139}$ of $\mathrm{HCl}$ to $\mathrm{Au}(111),{ }^{133}$ of $\mathrm{CO}_{2}$ to $\mathrm{Ni}(100),{ }^{62}$ of $\mathrm{CHD}_{3}$ on $\mathrm{Cu}(111),{ }^{63}$ and $\mathrm{of}_{2}$ to $\mathrm{Cu}(111), \mathrm{Cu}(100)$, and $\mathrm{Cu}(110) .{ }^{467}$ In essence everything that applies to DFMD also applies to QCT calculations using HDNNPs. For instance, just like energy dissipation away from the reaction zone can be modeled with DFMD using an Anderson thermostat, the same should be possible with the QCT-HDNNP approach. 
Finally, probably the best approach to the classical modeling of the effect of surface atom dynamics and $T_{\mathrm{S}}$ is the QM/Me embedding scheme of Meyer and Reuter. ${ }^{96}$ With this method, a small DFMD cell containing the projectile and the nearest surface atoms is embedded in a larger cell also containing metal atoms, which interact through many-body classical interatomic potentials. ${ }^{545}$ The QM/Me method enjoys all the advantages of the DFMD and QCT-HDNNP approaches. Additionally, energy dissipation away from the reaction zone is modeled even more accurately with this approach, because the surface unit cell can be taken large also in the directions parallel to the surface. The QM/Me method has been used to successfully study the energy dissipation accompanying DC of $\mathrm{O}_{2}$ on $\mathrm{Pd}(100) .{ }^{96}$ An advantage of the $\mathrm{QM} / \mathrm{Me}$ method is that the phonon excitation spectrum can be modeled with much higher energy resolution, making it possible to investigate the effect of the specific type of surface phonon (for instance, Rayleigh or other) on the reaction dynamics. This has recently been used successfully to explain differences between the diffusion dynamics of hot O-atoms on different Pd facets on the basis of differences between the phonon-mode-specific energy transfer to the different facets. ${ }^{546}$

The properties of the various implementations of the BOMS model are summarized in Table 4. The SO, SM, GLO, VTSR, MGLO, LRS, and PSA models in principle describe the moleculephonons coupling through a single atom oscillator, although additive electronic couplings to the motion of more than one surface atom have been taken into account with the LRS model. ${ }^{78}$ The SO, SM, and GLO models are only applicable to systems with no or weak electronic coupling, while the VTSR and PSA models do not describe mechanical coupling. When applied with QD, an advantage of the a posteriori SM, VTSR, and LRS schemes is that they do not add an extra DOF to be explicitly modeled in the dynamics calculations. The SCM model treats electronic coupling to several surface atom oscillators in a static way. The DFMD, HDNNP, and QM/Me schemes also treat the phonon fine-structure of the surface, and are capable of doing this to an increasing extent. However, these three schemes add many DOFs to the calculations, and cannot be used in conjunction with QD. QD schemes in which phonons are either treated as a heat bath with an open-system density matrix approach or a full wave function approach are available, but the cost of especially the latter approach scales unfavorably with the number of DOFs modeled in the dynamics calculations (for a brief discussion of these schemes and new approaches see ref. 547). This currently precludes their use in schemes aimed at obtaining chemically accurate $E_{\mathrm{b}}$.

4.1.3. The NBOSS model. In the NBOSS model, the BO approximation is abandoned, but the surface is treated as static. Currently, two types of methods can be used with dynamics calculations modeling motion in at least six DOFs (as appropriate for DC of the smallest, i.e., diatomic molecule) to compute $S_{0}$. The first type of method is the electronic friction (EF) method, ${ }^{54,57,58}$ which may be viewed as an extreme version of the mean field Ehrenfest method with the potential averaged over multiple ehp states taken equal to the electronic ground state potential. The second type of method is the independent electron surface hopping (IESH) method, ${ }^{128}$ where the molecule can change its electronic state and additionally ehp excitation is possible. In the above two methods, energy dissipation is directly coupled to nuclear motion, i.e., electronic excitation directly affects the nuclear motion. Methods also exist in which the molecule's motion on the surface is propagated adiabatically and conclusions are drawn about ehp excitation spectra and non-adiabatic energy loss to the surface (e.g., ref. 548 and ref. 549). While these methods may yield very useful insights (also regarding the importance of ehp excitation for specific DC systems ${ }^{97}$ ), we will not consider these methods in detail here.

EF theory ${ }^{550,551}$ can be derived using time-dependent perturbation theory. Starting with a mixed quantum-classical description of the electron-nuclear system, a formalism can be derived in which the electrons are dealt with through a generalized Langevin treatment, leading to a generalized Langevin equation for the nuclear DOFs. ${ }^{266}$ This scheme is valid if the weak coupling approximation is valid. ${ }^{266}$ In addition, the Markov approximation is invoked, thereby assuming short electronic coherence times. ${ }^{266,549}$ In EF dynamics the system conceptually moves on the BO ground state potential, and ehp excitation is described through a dissipative friction force and temperature dependent fluctuation forces, as mediated by friction coefficients. $^{266}$

Table 4 For the implementations of the BOMS model, shown is whether electronic coupling and/or mechanical coupling are described, and if and which couplings are described instantaneously in the dynamics. Also provided is information on whether the implementation adds DOFs to the dynamical model, and if so how many, on whether energy dissipation and local thermal fluctuations can be described, if and to what extent phonon fine structure can be described, and on the computational expense of the implementation

\begin{tabular}{llllllll}
\hline Method & El. coup. & Mech. co. & Instantaneous & Extra DOF & Dissipation fluctuations & Fine structure & Expensive \\
\hline SO & No & Yes & Mechanical & $1-3$ & Yes & No & In QD \\
SM & No & Yes & No & No & No & No & No \\
GLO & No & Yes & Mechanical & $2-6$ & Yes & No & No \\
VTSR & Yes & No & No & No & No & No & No \\
MGLO & Yes & Yes & Yes & $2-6$ & No & No \\
LRS & Yes & Yes & No & No & No & Medium \\
PSA & Yes & No & No & No & No & No \\
6 + mD QD & Yes & Yes & Yes & 1 so far & No & Very \\
SCM & Yes & No & No & No & No & No \\
DFMD & Yes & Yes & Yes & Many & Yes & Mes & Medium \\
QCT on HDNNP & Yes & Yes & Yes & Many & Yes & Medium-high & Medium \\
QM/Me & Yes & Yes & Yes & Many & High & Very
\end{tabular}


Presently, EF theory comes in two forms. The most straightforward version of EF theory is the local density friction approximation (LDFA). ${ }^{54}$ In this approach, the isotropic friction coefficient affecting motion of atom $i$ at location $r$ in the molecule is written as

$$
\eta_{i}(\underline{r})=\frac{3 \hbar}{r_{\mathrm{s}}^{2}(\underline{r})}\left(\frac{4}{9 \pi}\right)^{1 / 3} \sum_{l=0}^{\infty}(l+1) \sin ^{2}\left[\delta_{l, i}\left(r_{\mathrm{s}}\right)-\delta_{l+1, i}\left(r_{\mathrm{s}}\right)\right]
$$

In eqn (13), $r_{\mathrm{s}}$ is the mean electron radius of the free electron gas (which in turn is a function of the electron density of the bare metal at $\underline{r})$, and the $\delta_{1, i}\left(r_{\mathrm{s}}\right)$ are the scattering phase shifts for the atom moving through a jellium with the associated electron density. ${ }^{54,552}$ eqn (13) can be used to compute a friction force that is linearly proportional to the velocity of the atom and the friction coefficient defined in eqn (13). Advantages of the LDFA model are that it rests on a firm theoretical basis when applied to atoms scattering from metals, ${ }^{553}$ and that friction coefficients are readily available from the literature. ${ }^{552,554}$ The electron density that is required to compute $r_{\mathrm{s}}$ and the phase shifts can be calculated with DFT and its dependence on the spatial coordinates can be fitted with NNs. ${ }^{434}$ As noted by Alducin et al., ${ }^{264}$ the LDFA method can also be derived using a nonperturbative approach. In this approach, one can use timedependent density functional theory (TDDFT) to describe nonadiabatic interactions between a moving atom or molecule and a metallic surface over the entire regime of atomic velocities (see also ref. 95 and ref. 555). As noted by Alducin et al. ${ }^{264}$ Salin et $a l .{ }^{556}$ have proven that the approach based on eqn (13) and the friction force discussed above constitutes the exact low velocity limit of TDDFT for an atom moving through jellium.

A notable disadvantage of the LDFA method is that it does not take into account the electronic structure of the molecule, and how this changes along the reaction coordinate, because the straightforward application of the LDFA implies the independent atom approximation. ${ }^{55}$ An attempt to fix this with a new approach within the LDFA framework has been made, ${ }^{557}$ but work on $\mathrm{H}_{2}+\mathrm{Cu}(111)$ has shown that this approach may not be applicable to all molecules interacting with metals. ${ }^{57}$

The electronic structure of the molecule (and of the metal) is taken into account in so-called orbital dependent friction (ODF) theory, ${ }^{57,551,558-560}$ and this is an advantage of the ODF method. In the ODF method, the friction coefficients, which are elements of a $N_{\mathrm{d}} \times N_{\mathrm{d}}$ friction tensor, where $N_{\mathrm{d}}$ is the number of nuclear DOFs subject to electronic friction, are computed using

$$
\eta_{i \alpha j \beta}^{\mathrm{ODF}}(\mathbf{R})=2 \pi \hbar \sum_{\mathbf{k} a b} g_{\mathbf{k} a b}^{i \alpha}(\mathbf{R})^{*} \cdot g_{\mathbf{k} a b}^{j \beta}(\mathbf{R}) \delta\left(\varepsilon_{\mathbf{k} a}-\varepsilon_{\mathrm{F}}\right) \delta\left(\varepsilon_{\mathbf{k} b}-\varepsilon_{\mathrm{F}}\right)
$$

Here, the indexes $i$ and $j$ refer to atoms $i$ and $j$, and $\alpha$ and $\beta$ to Cartesian directions. Furthermore, $g_{\mathbf{k} a b}^{i \alpha}(\mathbf{R})$ is an electronphonon matrix element describing the non-adiabatic coupling between two electronic states of the system (the molecule plus the metal) with band indices $a$ and $b$ at wave vector $\mathbf{k}$ due to the motion of adsorbate atom $i$ along direction $\alpha$. Finally, the $\varepsilon_{\mathbf{k}}$ is the energy of the electron with band index a at wave vector $\mathbf{k}$, and $\varepsilon_{\mathrm{F}}$ is the energy of the Fermi level. A disadvantage of the ODF method is that it seems to be built on a contradiction. The quasi-static limit invoked in the derivation of eqn (14) would suggest to take the $\delta$-functions as narrow as possible, which would however imply that the resulting friction coefficients would go to zero. ${ }^{561}$ Maurer et $a .^{58}$ have argued that a finite width of the broadening functions can instead be used to include non-Markovian effects due to finite coherence times of the excited ehps. ${ }^{549}$ In practice the problem has been solved pragmatically by performing the calculations with varying width parameters and demonstrating that the results are rather insensitive to the widths employed over a range including the width parameter used in the actual calculations. ${ }^{57,58}$

Until recently ODF coefficients were only used in lowdimensional scattering calculations ${ }^{562-565}$ because techniques were lacking for their efficient evaluation. Only recently $6 \mathrm{D}$ friction tensors have become available for dynamics simulations on DC of diatomic molecules on metals (i.e., $\mathrm{H}_{2}+\mathrm{Ag}(111)^{58,64}$ and $\mathrm{Cu}(111),{ }^{57}$ and $\left.\mathrm{N}_{2}+\mathrm{Ru}(0001)^{59}\right)$. Diagonal elements of the friction tensor for motion of the molecule towards the surface and for vibrational motion are presented in the upper and lower panels of Fig. 12 for $\mathrm{H}_{2}+\mathrm{Cu}(111)^{57}$ and $\mathrm{N}_{2}+\mathrm{Ru}(0001),{ }^{59}$ respectively. These figures clearly show that the friction coefficients for vibrational motion show a much steeper increase going along the reaction path to the TS in the ODF than in the LDFA method. This steeper increase has in the past been the basis for making the argument that electronic structure effects should be taken into account when computing friction coefficients, ${ }^{55}$ and that ehp excitation should have an important effect on reactive scattering. ${ }^{55,564}$

Molecular dynamics with electronic friction (MDEF) ${ }^{551,558}$ calculations using the LDFA have now been performed for a number of DC systems, including $\mathrm{H}_{2}+\mathrm{Cu}(110),{ }^{54} \mathrm{Cu}(111),{ }^{57,174}$

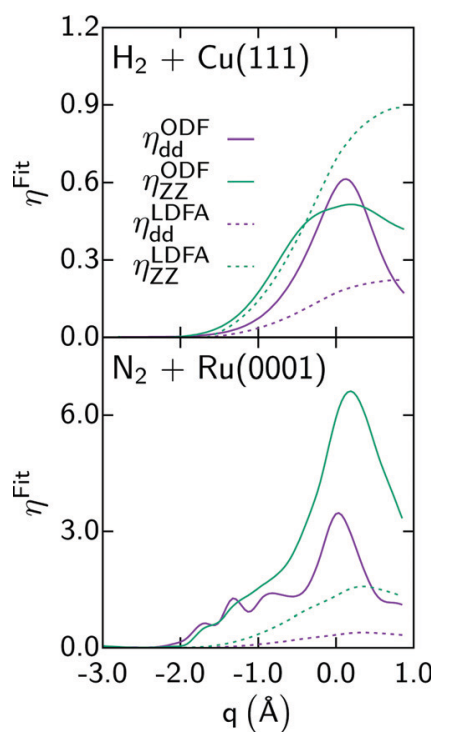

Fig. 12 Diagonal elements of the friction tensor, $\eta_{q q}$ (in $m e V$ ps $\AA^{-2}$ ), are shown as a function of the reaction coordinate $q$ ( $d$ is the bond distance of the molecule, $Z$ the distance to the surface) for the LDFA and the ODF approximations, as computed for $\mathrm{H}_{2}+\mathrm{Cu}(111)^{57}$ and $\mathrm{N}_{2}+\mathrm{Ru}(0001) .^{59}$ Data taken from ref. 57 and ref. 59. 
$\mathrm{Cu}(211),{ }^{511} \mathrm{Ag}(111),{ }^{58} \mathrm{Pt}(211),{ }^{48}$ and $\mathrm{Ru}(0001),{ }^{140} \mathrm{~N}_{2}+\mathrm{W}(110),{ }^{54}$ $\mathrm{HCl}+\mathrm{Au}(111),{ }^{85} \mathrm{H}_{2} \mathrm{O}+\mathrm{Ni}(111),{ }^{122}$ and $\mathrm{CH}_{4}+\mathrm{Ni}(111) .{ }^{99}$ So far, these calculations have suggested that ehp excitation can be rather safely neglected when computing $S_{0}$ (energy shifts of sticking curves usually being smaller than $\left.1 \mathrm{kcal} \mathrm{mol}^{-1}\right)$. This is not the case for hot-atom recombination of $\mathrm{H}$-atoms on $\mathrm{W}(110)$, where a hot $\mathrm{H}$-atom may dissipate its energy quite fast when ehp excitation is allowed, thereby making the hot-atom reaction less efficient. ${ }^{566}$ The comparisons between NBOSS calculations with the LDFA and with ODF that are now available suggest that, at least for $\mathrm{H}_{2}+$ metal systems $\left(\mathrm{H}_{2}+\mathrm{Ag}(111)^{58,64}\right.$ and $\left.\mathrm{Cu}(111)^{57}\right)$, it hardly matters whether the LDFA or ODF is used when computing DC probabilities (see Fig. 13 for $\mathrm{H}_{2}+\mathrm{Cu}(111)$ ). However, MDEF calculations with the LDFA and ODF show marked differences in their predictions of probabilities for vibrational de-excitation and accompanying non-adiabatic energy loss to the surface for scattering of $\mathrm{H}_{2}+\mathrm{Cu}(111) .{ }^{57}$ It should also be of interest to test the LDFA and ODF approaches on energy losses and vibrational de-excitation probabilities measured for $\mathrm{H}_{2}$ scattering from $\mathrm{Cu}(100) .{ }^{567,568}$

Non-adiabatic transitions between different electronic states of the molecule have also been implicated in DC of $\mathrm{O}_{2}$ on $\mathrm{Al}(111),{ }^{569,570}$ and in vibrationally inelastic scattering of NO from $\mathrm{Au}(111)^{571}$ and from cesiated $\mathrm{Au}(111),{ }^{230,231}$ with very solid evidence having been presented in the latter case. The evidence concerning the impact of electronically non-adiabatic effects (i.e., surface induced spin-orbital coupling between the triplet and singlet states of $\mathrm{O}_{2}$ ) on dissociation of $\mathrm{O}_{2}$ on $\mathrm{Al}(111)$ is controversial. ${ }^{399}$ Therefore, efforts have been made to find fingerprints of the non-adiabatic mechanism in the scattering of singlet $\mathrm{O}_{2}$ from $\mathrm{Al}(111)$ studied with theory. ${ }^{572,573}$ Calculations ${ }^{572,573}$ employing the fewest switches surface hopping method of Tully ${ }^{574}$ have led to predictions ${ }^{573}$ that significant amounts of the $\mathrm{O}_{2}$ incident in the excited singlet state should be reflected in the ground state triplet state. To our knowledge, this prediction has not yet been tested in experiments.

Tully and co-workers ${ }^{128,575}$ have extended the fewest-switching surface hopping method to deal with the case that ehp excitation

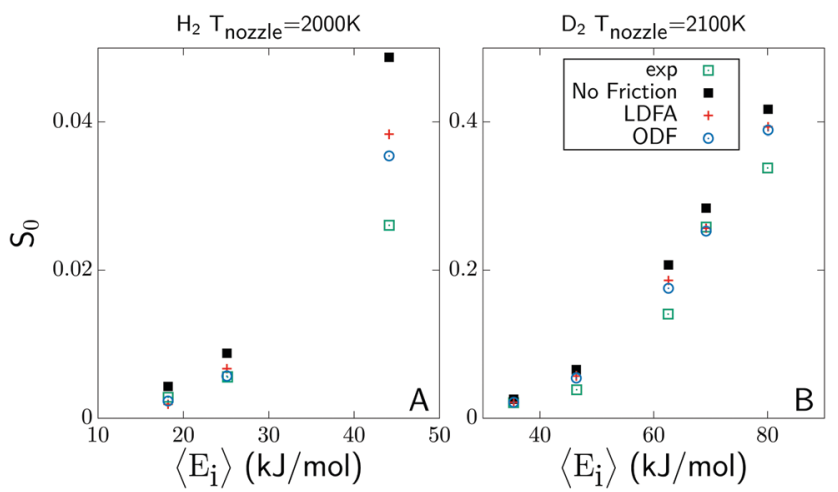

Fig. 13 Calculated ${ }^{57}$ and measured ("exp") ${ }^{12,641} S_{0}$ for sticking of normally incident $\mathrm{H}_{2}(\mathrm{~A})$ and $\mathrm{D}_{2}$ (B) on $\mathrm{Cu}(111)$ are shown as a function of average normal incidence energy. Computed results are shown for electronically adiabatic calculations ("No Friction"), and for calculations modeling ehp excitation using the LDFA ("LDFA") and ODF ("ODF"). Data taken from ref. 57. can accompany electronic transitions of the system (e.g. electron transfer leads to the molecule becoming ionic). The resulting IESH method ${ }^{128,575}$ has been used in numerous recent calculations on scattering of molecules like NO from metal surfaces. However, to our knowledge the method has not yet been used for DC on a metal surface, for which the method's accuracy remains to be proven.

As noted in ref. 266 a clear influence of electronically nonadiabatic effects on DC probabilities of molecules on metals remains to be established, whereas pronounced surface phonon effects are now known for a number of reactions (see above). A clear influence of electronically non-adiabatic effects on scattering of molecules back to the gas phase has been established (see above). ${ }^{230,231}$ Similarly, large non-adiabatic effects have been predicted ${ }^{189}$ and measured for scattering of atoms from surfaces. ${ }^{192,265,576}$ However, also due to the importance of heterogeneous catalysis the search for non-adiabatic effects on reactions on metals continues. ${ }^{266}$

4.1.4. The NBOMS model. In the NBOMS model, both nonadiabatic effects and surface atom motion are modeled. So far, in the calculations that do not use a density matrix approach and that we are aware of, the non-adiabatic effects have, with few exceptions, ${ }^{59,577}$ been modeled exclusively with EF within the LDFA approach. ${ }^{54}$ The calculations published differ with respect to how surface atom motion was modeled. The first publication studying both effects of surface atom motion and ehp excitation that we are aware of used an empirical potential model, and EF within the ODF approach. ${ }^{266,577}$ The paper ${ }^{577}$ addressed scattering of $\mathrm{CO}$ from $\mathrm{Cu}(100)$ and found the effects of ehp excitation on the sticking and on energy loss in scattering to be much smaller than the effects of surface phonon excitation. The study employed the MDEF method, a high-dimensional PES, and a generalized Langevin approach for describing dissipation and thermal fluctuations associated with ehp excitation.

Calculations using a GLO approach to describe the surface phonons and EF within the LDFA have addressed scattering of $\mathrm{N}_{2}$ from $\mathrm{W}(110)$, dissociative and non-dissociative adsorption of $\mathrm{N}_{2}$ on $\mathrm{W}(100),{ }^{143}$ scattering of $\mathrm{N}_{2}$ from $\mathrm{W}(100),{ }^{146}$ reactive scattering of $\mathrm{CO}_{2}$ from $\mathrm{Ni}(100),{ }^{69}$ of $\mathrm{N}$-atoms from $\mathrm{Ag}(111),{ }^{109,578,579}$ vibrational excitation and sticking of $\mathrm{H}_{2}$ on $\mathrm{Cu}(111),{ }^{168}$ and the Eley-Rideal recombination of $\mathrm{H}_{2}$ and $\mathrm{N}_{2}$ on tungsten surfaces. $^{52,580}$ In most studies energy dissipation to phonons was found to be more important than dissipation through ehp excitation, ${ }^{69,109,143,146,578,579}$ with ehp excitation being the more important channel only for $\mathrm{H}_{2}$ recombination. ${ }^{52,580}$ For $\mathrm{H}_{2}$ on $\mathrm{Cu}(111)$ ehp excitation needed to be included to describe the trend that the vibrational excitation probability from the vibrational state $v=0$ to $v=1$ increases with $T_{\mathrm{s}},{ }^{168}$ a trend also found for scattering of $\mathrm{CO}$ from $\mathrm{Cu}(100)$ in the early work of Tully and coworkers, ${ }^{577}$ and in experiments on $\mathrm{H}_{2}+\mathrm{Cu}(111) .{ }^{581}$ However, allowing dissipation to ehps (and to phonons) diminished the vibrational excitation probabilities relative to simulations with the BOSS model for $\mathrm{H}_{2}+\mathrm{Cu}(111) .{ }^{168}$

Calculations combining DFMD to describe surface atom motion with LDFA electronic friction (DFMDEF) were performed on vibrational excitation and sticking of $\mathrm{H}_{2}$ on $\mathrm{Cu}(111),{ }^{168}$ DC on and scattering of $\mathrm{HCl}$ from $\mathrm{Au}(111),{ }^{147}$ and DC of $\mathrm{N}_{2}$ 
on $\mathrm{Fe}(110){ }^{74,582}$ DFMDEF calculations also addressed energy dissipation upon DC of $\mathrm{H}_{2}$ on $\mathrm{Pd}(100) .{ }^{74,114,582}$ Another topic addressed with DFMDEF is Eley-Rideal reactions, of $\mathrm{H}$-atoms on $\mathrm{Cu}(111),{ }^{583}$ of D-atoms with $\mathrm{CD}_{3}$ pre-adsorbed to $\mathrm{Cu}(111),{ }^{584}$ and of $\mathrm{H}$ with $\mathrm{Cl}$ preadsorbed to $\mathrm{Au}(111) \cdot{ }^{585}$ Finally, DFMDED calculations investigated scattering of N-atoms from $\mathrm{Ag}(111),{ }^{74,541,582}$ and dynamic displacement of $\mathrm{CO}$ pre-adsorbed to $\mathrm{Cu}(111)$ by incident $\mathrm{H}$-atoms. ${ }^{586}$

The DFMDEF calculations on vibrational excitation of $\mathrm{H}_{2}$ on $\mathrm{Cu}(111)$ showed that the GLO + LDAF friction method is remarkably accurate in its description of vibrational excitation, at least within the limits of quasi-classical dynamics. ${ }^{168}$ The DFMDEF calculations on energy dissipation upon dissociation of $\mathrm{H}_{2}$ on $\operatorname{Pd}(100)^{74,114,582}$ showed that energy dissipation to ehps may be quite important in DC of $\mathrm{H}_{2}$. However, it is mainly important for the energy dissipation that occurs after the dissociation, when the $\mathrm{H}$-atoms separate. DFMDEFp calculations on energy loss of hyperthermal $\mathrm{H}$-atoms scattering from $\mathrm{Cu}(111)$ and $\mathrm{Au}(111)$ predicted that $\mathrm{H}$ loses much more energy $(\approx 1 \mathrm{eV})$ to ehp excitation than to phonons $(0.38 \mathrm{eV}$ on $\mathrm{Cu}(111)$, and $0.14 \mathrm{eV}$ to $\left.\mathrm{Au}(111)^{189}\right)$. In these calculations energy loss to ehp excitation was computed a posteriori from DFMD calculations (hence the "p" in DFMDEFp). ${ }^{189}$ The prediction was later confirmed in experiments of Bünermann et al. ${ }^{192}$

The tendency of $\mathrm{H}$-atoms to lose energy to ehps also explains the importance of ehp excitation to hot-atom recombination of $\mathrm{H}$-atoms on tungsten surfaces. ${ }^{52,566,580}$ Novko et al. worked on surface electron density models for AIMDEF simulations, asking the question of how to describe the effect of the electron density associated with a mobile surface on ehp excitation efficiently and accurately. They found ${ }^{74}$ that this is best done with a Hirshfeld partitioning scheme first used for this purpose by Reuter and coworkers. ${ }^{557}$ Comparison of DFMDEF to DFMD results for $\mathrm{DC}$ of $\mathrm{HCl}$ on $\mathrm{Au}(111)$ showed only a small difference, implying that ehp excitation does not greatly affect the $S_{0}$ for this system. ${ }^{147}$

Recently MDEF calculations have emerged in which the HDNNP method describing the coupling of molecular and phonon motion was combined with modeling ehp excitation with EF. For molecules this was first done for $\mathrm{HCl}+\mathrm{Au}(111)$ using EF at the LDFA level. ${ }^{133}$ For this system, ehp excitation was found to have only a small effect on reaction (similar to the DFMDEF result ${ }^{147}$ ), vibrational excitation, and energy transfer to the surface. ${ }^{133} \mathrm{~A}$ similar study of $\mathrm{N}_{2}$ on $\mathrm{Ru}(0001)$ only found a small effect of LDFA friction on sticking and scattering. ${ }^{138}$ Interestingly, a much larger effect of ehp excitation on DC of $\mathrm{N}_{2}$ on $\mathrm{Ru}(0001)$ was found using the ODF (Fig. 14). ${ }^{59}$ In contrast to the $\mathrm{H}_{2}+\mathrm{Cu}(111)$ case $^{57}$ discussed in Section 4.1.3 (see Fig. 13), now a big difference is found between the sticking results depending on whether the LDFA or ODF friction is used: for $\mathrm{N}_{2}+\mathrm{Ru}(0001)$, adding ehp excitation to the model with the ODF roughly halves $S_{0}$, whereas little effect is found with the LDFA. Using the ODF also led to better agreement with the best experimental estimates of $S_{0} \cdot{ }^{59}$ The reason that ODF friction has a much larger effect than LDFA friction on reactive scattering of $\mathrm{N}_{2}$ from $\mathrm{Ru}(0001)$ is that the diagonal ODF friction coefficients for motion towards the surface and in the molecular vibration

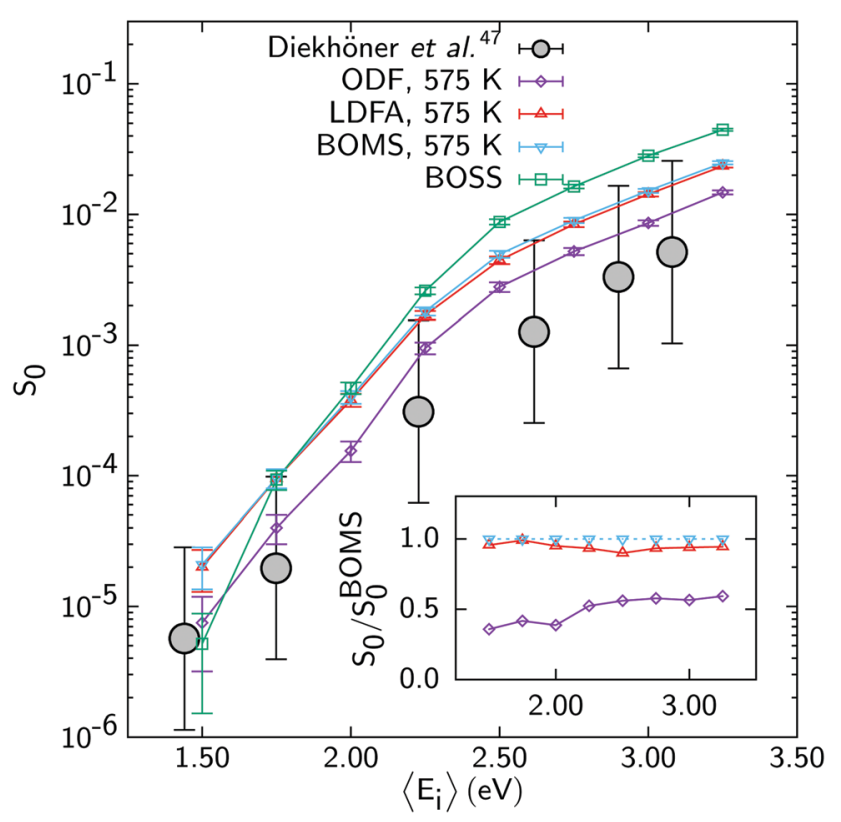

Fig. $14 S_{0}$ computed for $N_{2}+R u(0001)$ with MDEF and the RPBE DF using the BOSS model, ${ }^{139}$ the BOMS model, ${ }^{139}$ and the NBOMS model with the LDFA and the ODF approximations ${ }^{59}$ are shown as a function of $E_{i}$. Experimental results are also shown. Taken from ref. 59 (https://pubs.acs. org/doi/10.1021/acs.jpclett.9b00523). Further permission requests to be directed to the ACS.

are both much larger than their LDFA counterparts, whereas this is not the case for $\mathrm{H}_{2}+\mathrm{Cu}(111)$ (see Fig. 12).

The MDEF method has been used also with a high-dimensional PES describing the coupling to phonons for atom-metal surface $(\mathrm{H}+\mathrm{Au}(111))$ scattering by Janke et al. ${ }^{180}$ and Bünermann et al. ${ }^{192}$ To obtain a high-dimensional PES, they used effective medium theory (EMT), ${ }^{180,190}$ basing the EMT fit on data obtained with the SRP48 DF for $\mathrm{H}_{2}+\mathrm{Cu}(111)$. Fitting the PES with EMT does not allow one to reproduce raw DFT data with chemical accuracy, but semi-quantitative accuracy can be achieved $^{190}$ and a considerable advantage of EMT for MDEF calculations is that it also yields directly the electronic density of the system, albeit it in this case for both the surface and the projectile. ${ }^{180}$ However, Janke et al. found that the dynamics results showed little dependence on whether the electron density of $\mathrm{H}$ was included when calculating the system's electronic density. ${ }^{180}$ The EMT-LDFA results were in excellent agreement with experiments for $\mathrm{H}+\mathrm{Au}(111),{ }^{192}$ suggesting that LDFA friction is accurate enough for describing scattering of hyperthermal $\mathrm{H}$-atoms from late TMs.

Finally, in DFMDEF calculations the contribution of the projectile to the system's electronic density can be removed with the Hirshfeld partitioning scheme ${ }^{557}$ mentioned earlier. This way, the effect of surface atom motion on the EF coefficient can be efficiently and accurately computed. ${ }^{74}$ As far as we know, this has not yet been implemented in a MDEF scheme based on HDNNPs, where the assumption made so far has been that the electronic density of the mobile surface equals that of the static surface. 
As also reviewed by Alducin et al. ${ }^{264}$ the conclusions from dynamics studies employing EF as obtained from the LDFA to study the effects of ehp excitation (and in some cases phonons) on molecule-metal surface reactions are that: (i) the effect of LDFA friction on sticking through DC tends to be small, as the fate of the molecule (dissociation or not) is usually decided before ehp excitation becomes important (ii) for activated dissociation ehp excitation inhibits dissociation, (iii) for trapping mediated dissociation ehp excitation may promote sticking, (iv) ehp excitation dominates energy dissipation by dissociated $\mathrm{H}$-atoms and is still important (though less so than phonons) for dissipation by heavier dissociated atoms, such as $\mathrm{N}$-atoms. Finally, $(v)$ the energy loss to ehp excitation is determined by the electron density in the regions the atoms travel through with high velocity, and the extent of these regions, and more generally (vi) ehp excitation usually becomes the more important the lighter the atoms in the molecule are. ${ }^{264}$ As noted above, at least one example has been found in which it matters whether ODF or the LDFA is used in calculations on sticking. ${ }^{59}$ More research is needed to investigate how conclusions $\mathrm{i}-\mathrm{v}$ are affected if ehp excitation is modeled with ODF instead of with the LDFA.

\subsection{CT methods}

In the CT method ${ }^{587-589}$ the classical equations of motion

$$
\begin{gathered}
\frac{\mathrm{d} x}{\mathrm{~d} t}=\frac{\partial H}{\partial p} \\
\frac{\mathrm{d} p}{\mathrm{~d} t}=-\frac{\partial H}{\mathrm{~d} q}=-\frac{\partial V}{\mathrm{~d} q}
\end{gathered}
$$

are solved. Several versions of the method have been applied to molecule-surface scattering. In the simplest method, the ordinary CT method, no zero-point vibrational energy (zpe) is given to the molecule at the onset of the trajectory. Initial zpe is however given to the molecule in the QCT method. Finally, in applications to diatomic molecules scattering from surfaces an amount of zpe in the vibration of the diatomic molecule that depends on the molecule-surface distance can be added to what becomes a $5 \mathrm{D}$ potential, ${ }^{590}$ in what has been called the CZPE method, to account for the softening of the bond when the molecule approaches the surface and to avoid zpe leakage.

In most applications to molecule-metal surface scattering, the QCT method is used rather than the CT or CZPE method. However, comparisons to QD results have shown that the CT method sometimes outperforms the QCT method for nonactivated reaction, ${ }^{591,592}$ and the CZPE method may work best for trapping-mediated reaction. ${ }^{590}$ Over the last decade, the CT method has been used to study DC of $\mathrm{H}_{2}$ on a tungsten surface partly covered by $\mathrm{Cu},{ }^{111}$ for reactive scattering of $\mathrm{H}_{2}$ from metals at grazing incidence, ${ }^{593}$ and for non-reactive scattering of $\mathrm{N}_{2}$ from the W(110) surface. ${ }^{110}$ In the study of reaction of $\mathrm{H}_{2}$ on a tungsten surface partly covered by $\mathrm{Cu}$ the CZPE method has been used as well. ${ }^{111}$

The QCT method can also be used to study state-to-state scattering. In this case a method needs to be used to assign a final rotational and vibrational state at the end of the scattered trajectory. This can be done using "histogram binning" (also called "standard binning"), in which the final state is assigned on the basis of the closest vibrational energy, rotational energy or vibrational action or angular momentum of the quantum state, and this is the method that is most often used (ref. 168 and ref. 174). It has been argued that a better method is to use the so-called Gaussian binning procedure (for details see e.g. ref. 594-596), and this method has also been used in applications to molecule-metal surface scattering. ${ }^{133,596}$

There are now numerous instances in which initial-state resolved reaction probabilities computed with QCT have been compared with QD results for a diatomic molecule reacting on a metal surface to assess the accuracy of QCT. Examples include $\mathrm{H}_{2}+\mathrm{Cu}(111),{ }^{159,597} \mathrm{Ru}(0001),{ }^{151} \mathrm{Ni}(111),{ }^{476} \mathrm{Pd}(111),{ }^{158}$ $\operatorname{Pt}(111),{ }^{156} \mathrm{Pt}(211),{ }^{48}$ and $\mathrm{D}_{2}+\mathrm{Ag}(111) .{ }^{186}$ Generally, very good agreement is obtained. As an example we show very recent results for $\mathrm{H}_{2}+\mathrm{Cu}(111)^{597}$ in Fig. 15, which also addressed $E_{i}$ much lower than the zpe corrected barrier height $E_{\mathrm{b}}^{\mathrm{c}}$. For these energies, the QCT reaction probabilities should be zero. As can be seen, the agreement between the QCT and QD reaction probabilities for the $(v=0, j=0)$ rovibrational ground state is excellent even for energies somewhat below $E_{\mathrm{b}}^{\mathrm{c}}(0.65 \mathrm{eV}$ for the PES used in ref. 597). Only near $0.50 \mathrm{eV}$ the QCT and QD results started to deviate, which the authors attributed to the neglect of tunneling effects in the QCT calculations. ${ }^{597}$

The recent work also compared QD and QCT "sticking probabilities" that were computed for a gas temperature of 300 K. In Fig. 15 these results also show substantial differences for $E_{i}=0.5 \mathrm{eV},{ }^{597}$ which might be taken to suggest that the QCT method is not accurate for simulating experimental $S_{0}$ for systems like $\mathrm{H}_{2}+\mathrm{Cu}(111)$. However, this is not correct. The quantities plotted for $300 \mathrm{~K}$ are not true $S_{0}$ in the sense that

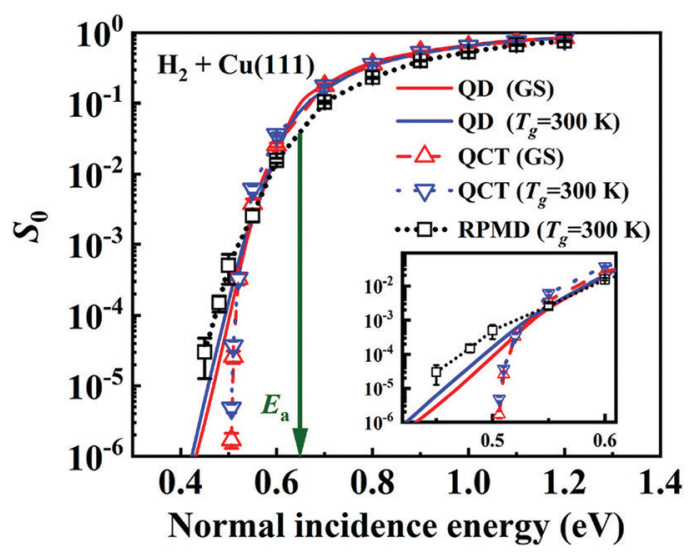

Fig. $15 S_{0}$ computed $^{597}$ for $\mathrm{H}_{2}+\mathrm{Cu}(111)$ with $\mathrm{QD}$ for the ground rovibrational state (GS, red solid line), with $Q D$ using Boltzmann averaging for a gas temperature $\left(T_{\mathrm{g}}\right)$ of $300 \mathrm{~K}$ (blue solid line), with QCT for the ground rovibrational state (GS, red upper triangle), with QCT using Boltzmann averaging for a $T_{\mathrm{g}}=300 \mathrm{~K}$ (blue lower triangle), and with RPMD for $T_{\mathrm{g}}=300 \mathrm{~K}$ (black squares) are shown as a function of $E_{\mathrm{n}}$. Reprinted with permission from (Q. H. Liu, L. Zhang, Y. Li, B. Jiang, Ring polymer molecular dynamics in gas-surface reactions: Inclusion of quantum effects made simple, J. Phys. Chem. Lett., 2019, 10, 7475-7481). Copyright (2019) American Chemical Society. 


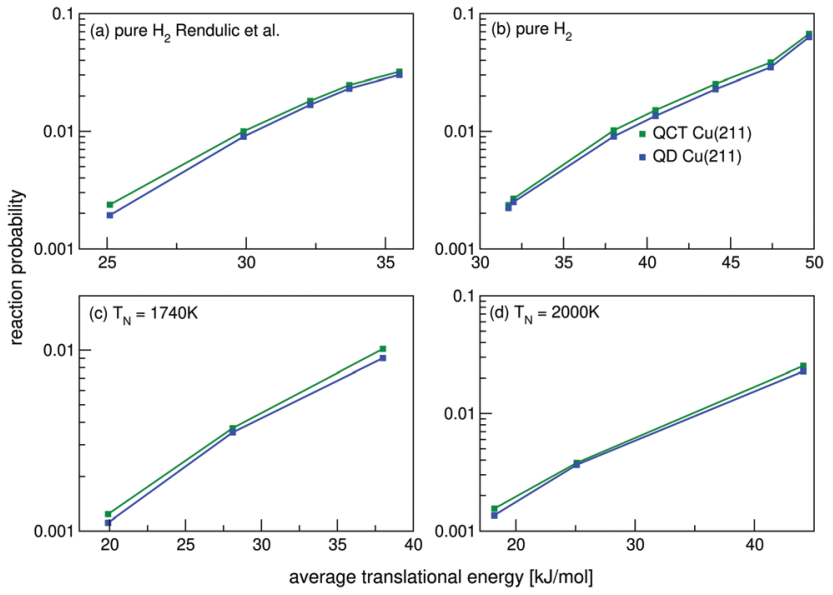

Fig. $16 \mathrm{~S}_{0}$ computed $^{511}$ with the SRP48 DF for $\mathrm{H}_{2}+\mathrm{Cu}(211)$ using QD (blue lines) and QCT (green lines), for beam conditions used in experiments ${ }^{641,649}$ on $\mathrm{H}_{2}+\mathrm{Cu}(111)$. Data taken from ref. 511.

they do not represent measurable quantities. As noted by the authors, ${ }^{597}$ achieving an $E_{i}$ of $0.50 \mathrm{eV}$ requires a $T_{\mathrm{N}}$ or gas temperature of $2100 \mathrm{~K}$ rather than $300 \mathrm{~K}$. Under high $T_{\mathrm{N}}$, but low $E_{i}$ conditions the sticking is usually dominated by vibrational states with $v>0$, for which QCT calculations accurately predict reaction probabilities. For this reason QCT usually accurately predicts $S_{0}$ under actual experimental conditions, as can be seen by the QD vs. QCT comparison for $\mathrm{H}_{2}+$ $\mathrm{Cu}(211),{ }^{511}$ for conditions under which experiments were carried out for $\mathrm{H}_{2}+\mathrm{Cu}(111)$ (see Fig. 16). As can be seen, for the "simulated experiments", excellent agreement is achieved between QCT and QD $S_{0}$.

It is much harder to assess the accuracy of the QCT method (also as it is used in the framework of DFMD calculations) for polyatomic molecules, but as we will now discuss in some detail such efforts have been made for $\mathrm{CH}_{4}+\mathrm{Ni}(111)^{500,598}$ and $\mathrm{Ni}(100),{ }^{598} \mathrm{CHD}_{3}+\mathrm{Ni}(111),{ }^{161}$ and $\mathrm{H}_{2} \mathrm{O}+\mathrm{Ni}(111) .{ }^{121,597} \mathrm{We}$ start with a QD vs. QCT comparison for $\mathrm{CH}_{4}$ on $\mathrm{Ni}(111)$, as made in the framework of a RPH model ${ }^{50,203}$ by Jackson and coworkers. ${ }^{598}$ QD and QCT results are compared in Fig. 17 for the vibrational ground state, and for the $1 \nu^{3}$ and $2 \nu^{3}$ states, respectively. The results were computed by carrying out a calculation for the impact site corresponding to the TS, and then averaging over impact sites and phonon coordinates and momenta using the LRS method discussed above. ${ }^{598}$ As can be seen, the QD and QCT results for the vibrationally excited states are in rather good agreement, but the agreement for the vibrational ground state is somewhat poorer, especially for $E_{i}<E_{\mathrm{b}}^{\mathrm{c}}\left(0.94 \mathrm{eV}\right.$ in this case $\left.{ }^{598}\right)$. The disagreement between the QD and QCT results was attributed to zpe violation, a problem that may be especially severe in this case as the methane molecule is carrying about $1.2 \mathrm{eV}$ of initial zpe with it. ${ }^{598}$

In practice, the agreement between QD and QCT results for an actual "sticking probability" is likely to be much better than suggested by Fig. 17, for two reasons: (i) the molecular "laser off" beam also contains vibrationally excited molecules, and their contribution to the $S_{0}$ should be more accurately evaluated

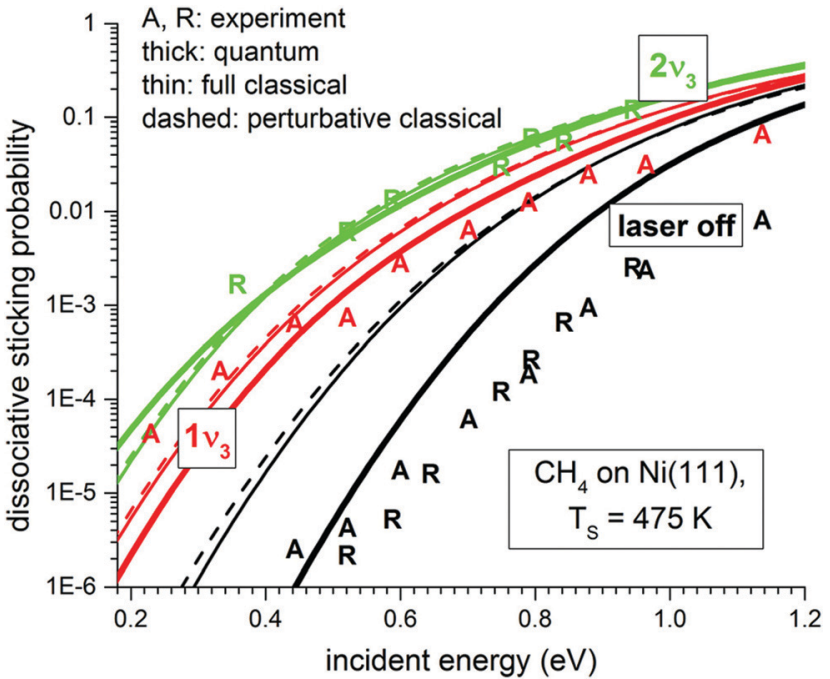

Fig. $17 S_{0}$ computed ${ }^{598}$ for $\mathrm{CH}_{4}+\mathrm{Ni}(111)$ using the LRS model for phonon coupling are shown as a function of incidence energy. Results are shown for methane initially in its rovibrational ground state (black), in its $1 \nu_{3}$ state (red) and in its $2 \nu_{3}$ state (green), also comparing to experimental results (same color coding) from the Utz group $(A)^{14}$ and the Beck group (R). ${ }^{635}$ The theoretical results were obtained with the RPH method (thick solid lines), the QCT method (thin solid lines), and the QCT method using a perturbative Hamiltonian (dashed lines). Reprinted from [M. Mastromatteo and B. Jackson, The dissociative chemisorption of methane on $\mathrm{Ni}(100)$ and $\mathrm{Ni}(111)$ : classical and quantum studies based on the reaction path Hamiltonian, J. Chem. Phys., 2013, 139, 194701], with the permission of AIP Publishing.

with QCT, as also clearly suggested by Fig. 17, and (ii) neither the QCT nor the QD calculations of Fig. 17 are full-dimensional. For instance, the individual QCT calculations are effectively 10D, as the $X$ and $Y$ DOFs for motion along the surface were taken out of the problem and later averaged over, and the rotational DOFs were treated with the rotationally adiabatic approximation (RAA) using QD. It is well known that the QCT method in principle produces increasingly more accurate results the more DOFs are taken into account and averaged over in the actual dynamics calculations. ${ }^{588}$ DFMD calculations on $\mathrm{CHD}_{3}+\operatorname{Pt}(111)$ for $E_{i}$ somewhat above $E_{\mathrm{b}}^{\mathrm{c}}$ found that in practice zpe violation contributed to reaction very rarely, so that in practice zpe violation is not expected to present a problem for $\mathrm{CH}_{4}+$ metal systems under conditions at which $E_{i}>E_{\mathrm{b}}^{\mathrm{c}}{ }^{51}$

In the same paper on $\mathrm{CHD}_{3}+\mathrm{Pt}(111),{ }^{51}$ the possible effect of the neglect of tunneling in QCT or DFMD calculations on $\mathrm{CH}_{4}$ dissociation on metals was also addressed. Early calculations employing a 1D mode ${ }^{599}$ had suggested such effects to be quite large. On the basis of RPH calculations discussed in the SI to ref. 51 it was possible to show that effects of tunneling should be minor for $\mathrm{CH}_{4}$ dissociating on $\mathrm{Pt}(111)$ even for $T_{\mathrm{S}}$ as low as $120 \mathrm{~K}$ (see also Fig. 18).

In the above QCT/QD comparison for $\mathrm{CH}_{4}+\mathrm{Ni}(111)$, the same dynamical models were used in the QCT and QD calculations, thereby comparing results of 10D dynamical calculations with additional but identical averaging procedures. ${ }^{598}$ This has the advantage of comparing "apples" with "apples", but in this case 


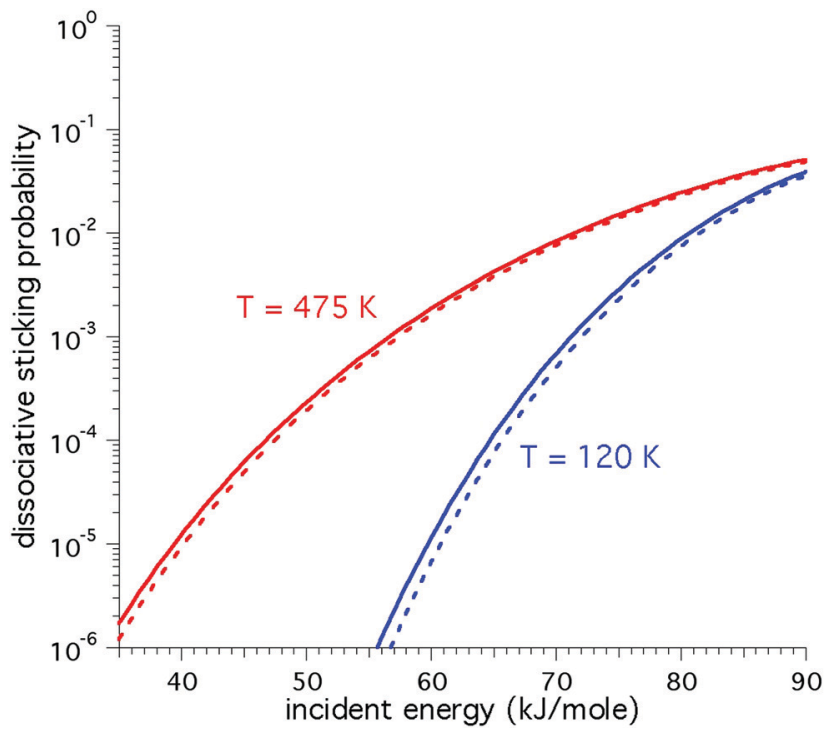

Fig. 18 Dissociative sticking probability as a function of normal incidence energy for vibrational ground state $\mathrm{CH}_{4}$ reacting with $\mathrm{Ni}(100)$ at $120 \mathrm{~K}^{51}$ and at $475 \mathrm{~K}^{50}$ The results were obtained ${ }^{50}$ with the reaction path method, for the case where the vibrationally non-adiabatic coupling are set equal to zero. The dashed lines exclude contributions to the sticking from tunneling. Reprinted with permission from (F. Nattino, H. Ueta, H. Chadwick, M. E. van Reijzen, R. D. Beck, B. Jackson, M. C. van Hemert and G. J. Kroes, Ab Initio Molecular Dynamics Calculations versus Quantum-State-Resolved Experiments on $\mathrm{CHD}_{3}+\mathrm{Pt}(111)$ : New Insights into a Prototypical GasSurface Reaction, J. Phys. Chem. Lett., 2014, 5, 1294-1299). Copyright (2014) American Chemical Society.

"stacks the deck" against QCT by limiting the number of dynamical DOFs, thereby worsening the performance of QCT. ${ }^{588}$ On the other hand, a full-dimensional QD vs. QCT comparison is presently not possible for $\mathrm{CH}_{4}+$ metal surface systems. Zhou and Jiang circumvented this issue by performing a comparison of QCT with experimental $S_{0}$ for $\mathrm{CHD}_{3}+\mathrm{Ni}(111),{ }^{161}$ using a $\mathrm{PES}^{167}$ obtained with a SRP-DF that was shown to yield chemical accuracy for conditions under which the QCT method was expected to be accurate. ${ }^{44}$ Zhou and Jiang tested the performance of the QCT method for conditions under which $E_{i}$ was below or just barely above $E_{\mathrm{b}}^{\mathrm{c}}$ for $\mathrm{CHD}_{3}+\mathrm{Ni}(111)$ (85.9 and $89.9 \mathrm{~kJ} \mathrm{~mol}^{-1}$ for $\mathrm{CH}$ and $\mathrm{CD}$ bond breaking, respectively). The calculations used a 15D dynamical model and PES, and the LRS method was used to take into account the effect of surface phonons and temperature. ${ }^{161}$ Results of the calculations are compared in Fig. 19. The mean distance (MD) from the computed $S_{0}$ to a fitted curve (not shown) through the experimental $S_{0}$ (Fig. 19) was $8 \mathrm{~kJ} \mathrm{~mol}^{-1}$, meaning that chemical accuracy was not achieved.

The authors attributed the discrepancy noted in Fig. 19 to zpe leakage causing too much reaction in the QCT calculations. However, it should be noted that the discrepancies get larger with increasing $E_{i}$, whereas better agreement would be expected instead. This is likely due to artificial intramolecular vibrational redistribution (IVR) that can already occur in vibrationally excited molecules while they fly through the gas phase with the use of QCT. Specifically, a problem not considered by the

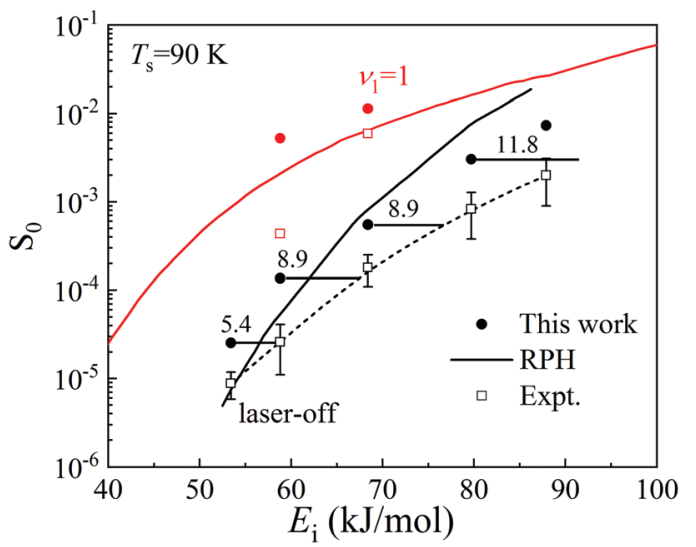

Fig. 19 Sticking probabilities computed ${ }^{161}$ for $\mathrm{CHD}_{3}+\mathrm{Ni}(111)$ with the QCT method and using the LRS model for surface atom motion for laser off reaction (black solid circles) and for reaction of $\nu_{1}=1 \mathrm{CHD}_{3}$ (red solid circles) are compared with experiment ${ }^{20}$ (black and red squares) for $T_{\mathrm{s}}=$ $90 \mathrm{~K}$. Also shown are results obtained for the same system and conditions using the RPH method. ${ }^{729}$ Reprinted by permission from Springer Nature Customer Service GmbH: Springer Nature, Sci. China Chem., Modespecific and bond-selective dissociative chemisorption of $\mathrm{CHD}_{3}$ and $\mathrm{CH}_{2} \mathrm{D}_{2}$ revisited using a new potential energy surface, $\mathrm{X}$. $\mathrm{Y}$. Zhou and $\mathrm{B}$. Jiang, vol. 61, pp. 1134-1142, Copyright 2018.

authors was that He-seeded beams were used in the experiments they compared to. ${ }^{20}$ This meant that nozzle temperatures of $550,600,700,730$, and $900 \mathrm{~K}$ had to be used ${ }^{20}$ to achieve the $E_{i}$ for which experimental results are shown in Fig. 19 (the authors did take the velocity distribution of the MBs into account, using the data presented in Table S1 of ref. 44). For $T_{\mathrm{N}} \geq 650 \mathrm{~K}$ the artificial IVR from D-atom excited vibrational states noted above may occur and enhance the computed $S_{0}$ for $\mathrm{CHD}_{3}$ reacting on metals. ${ }^{44}$ Artificial IVR (as it may occur in the gas phase with QCT) was not a problem in the previously discussed QCT-QD comparison in which the RPH method mo,203 $^{50}$ was used, as energy will not flow between pure harmonic oscillator states if the PES is expanded harmonically with respect to the reaction path, as was done. ${ }^{598}$ The above means that the QD-QCT comparison of Fig. $19^{161}$ might have been more favorable had the comparison been to experiments in which $\mathrm{H}_{2}$ seeded $\mathrm{CHD}_{3}$ beams were used, in which lower $T_{\mathrm{N}}$ can be used to achieve given $E_{i \cdot}{ }^{44}$

Finally, a very recent comparison between 7 + 2D QD and 9D QCT results was made for $\mathrm{DC}$ of $\mathrm{D}_{2} \mathrm{O}$ on $\mathrm{Ni}(111) .{ }^{597}$ With "7 + 2D QD", we mean that a 9D PES was used, and that 7D QD calculations performed for 15 impact sites were used to obtain approximate 9D QD results using the SAED approximation. As can be seen from Fig. 20, QCT calculations of the reaction probability of the rovibrational ground state of $\mathrm{D}_{2} \mathrm{O}$ considerably overestimated the QD result for the same state, even for reaction probabilities considerably greater than 0.01 and $E_{i} \gg E_{\mathrm{b}}^{\mathrm{c}}$ (which is $0.58 \mathrm{eV}$ with the PES used ${ }^{597}$ ).

The discrepancy noted above was attributed to zpe leakage. ${ }^{597}$ It is not clear why the discrepancy is so large for $\mathrm{D}_{2} \mathrm{O}+\mathrm{Ni}(111)$, while much better agreement was obtained for $\mathrm{CHD}_{3}+\mathrm{Ni}(111)$ (see Fig. 19). Possible reasons are that (i) there is no averaging ${ }^{588}$ 


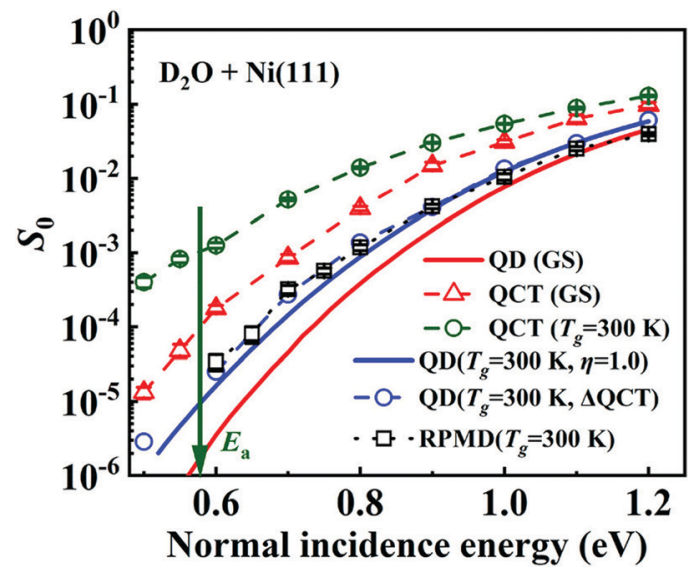

Fig. $20 S_{0}$ computed with QD for the ground rovibrational state (GS, red line), with QCT for the ground rovibrational state (GS, red triangles and dashed red lines), with QCT for a gas temperature of $300 \mathrm{~K}$ (green circles, dashed lines), with QD for a gas temperature of $300 \mathrm{~K}$ assuming a vibrational efficacy of 1.0 (blue line), with $Q D$ at a gas temperature of $300 \mathrm{~K}$ assuming the difference with QD at $0 \mathrm{~K}$ to be the same as with QCT (blue circles and blue dashed lines) are compared to values computed with the RPMD method. ${ }^{597}$ Reprinted with permission from (Q. H. Liu, L. Zhang, Y. Li and B. Jiang, Ring polymer molecular dynamics in gas-surface reactions: Inclusion of quantum effects made simple, J. Phys. Chem. Lett., 2019, 10, 7475-7481). Copyright (2019) American Chemical Society.

over initial states of the molecule and surface phonons in the calculation on $\mathrm{D}_{2} \mathrm{O}+\mathrm{Ni}(111)$, (ii) the SAED approximation may not work for $\mathrm{D}_{2} \mathrm{O}+\mathrm{Ni}(111)$ (this approximation has only been shown to work for reaction probabilities $>0.01$ in comparisons with actual 9D QD results for $\mathrm{H}_{2} \mathrm{O}+\mathrm{Cu}(111)^{61}$ and $\left.\mathrm{Ni}(100)\right)$. In the framework of this comparison, a point of confusion may be that $6 \mathrm{D}$ reaction probabilities for the vibrational ground state and the three fundamental vibrationally excited states seemed to come out larger with QD than with QCT for $\mathrm{D}_{2} \mathrm{O}+\mathrm{Ni}(111)$ (see Fig. S6 of ref. 121). However, it turns out that the 6D QCT dynamics results shown in Fig. S6 of ref. 121 were obtained with the more repulsive, empirically scaled PES developed for $\mathrm{D}_{2} \mathrm{O}+\mathrm{Ni}(111)$, while the QD calculations shown were done with a PW91 PES. ${ }^{600}$ In any case we suggest that more research be done on comparing QCT with QD for DC of $\mathrm{H}_{2} \mathrm{O}$ on metals, preferably comparing 9D QCT to 9D QD results for systems for which the latter results are available. ${ }^{61,98}$ For the moment we note that the present results for $\mathrm{D}_{2} \mathrm{O}+\mathrm{Ni}(111)$ (Fig. 20) cast doubt on the ability of the QCT and DFMD methods to reliably model DC of water on metals.

As already noted Gaussian binning can be used to improve the accuracy of QCT calculated state-to-state scattering probabilities. Interestingly, recent work has shown that this method may also be used to improve the accuracy of computed $S_{0}$, for $\mathrm{H}_{2}+\mathrm{Pd}(111) .{ }^{596}$ In this procedure, the sticking probability is calculated according to weighting scheme $X$ using

$$
S_{0}^{\mathrm{X}}=\frac{N_{\mathrm{S}}}{N_{\mathrm{S}}+\Sigma_{\mathrm{R}}^{\mathrm{X}}} .
$$

In eqn (16), $N_{\mathrm{S}}$ is the number of trajectories counted as reactive, and $\sum_{\mathrm{R}}^{\mathrm{X}}$ is the total weight of the $N-N_{\mathrm{S}}$ nonreactive trajectories according to weighting scheme $\mathrm{X}$. In the weighting scheme used,

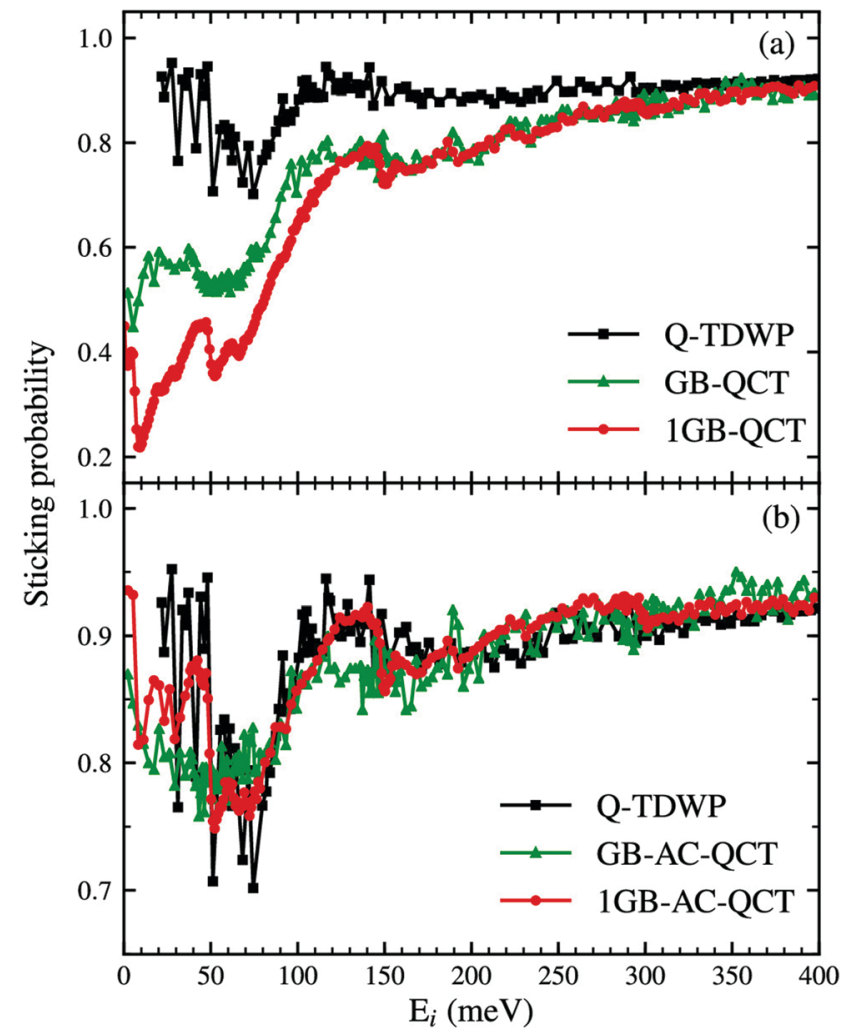

Fig. 21 Sticking probabilities computed ${ }^{596}$ with QCT dynamics using different Gaussian binning schemes ("GB" and " $1 \mathrm{~GB}$ ", see the text) without (panel a) and with (panel B) the adiabaticity correction ("AC") are compared with values computed using QD (i.e., with the TDWP method). Taken from ref. 596 (https://pubs.acs.org/doi/10.1021/acs.jpclett.9b02742). Further permission requests to be directed to the ACS.

weights may be assigned according to the final actions (for vibration, rotation, and diffraction, called "GB" in Fig. 21), or on the basis of the final total internal and parallel translational energy (called "1GB" in Fig. 21). In addition it is necessary to apply a so-called adiabaticity correction for reactions that may be trapping mediated ("AC" in Fig. 21), as explained in ref. 596, ref. 601 and ref. 602. Especially calculations using the GB-AC-QCT scheme are in excellent agreement with QD results for the initialstate resolved reaction probability of $(v=0, j=0) \mathrm{H}_{2}$ with $\operatorname{Pd}(111)$ (see Fig. 21b, $j$ is the rotational quantum number of $\mathrm{H}_{2}$ ). ${ }^{596}$ In contrast, reaction probabilities computed with the ordinary QCT method decreased monotonically with $E_{i}$ (see Fig. 1 of ref. 596). Probabilities for rotationally (in)elastic scattering from $v=0$, $j=0$ to $v=0, j=0,2$, and 4 were in excellent agreement with QD results using the 1GB-AC-QCT scheme (see Fig. 3 of ref. 596). The 1GB-AC-QCT scheme describes sticking of $\mathrm{H}_{2}$ on and rotationally inelastic scattering of $\mathrm{H}_{2}$ from $\mathrm{Pd}(111)$ just as well as or even better for $(v=0, j>0)$ than for $(v=0, j=0) \mathrm{H}_{2} \cdot{ }^{603}$ It would be nice if this scheme could be extended to polyatomic molecules reacting on metal surfaces.

Finally, it is also possible to compare vibrational efficacies, which measure how efficiently pre-exciting to a vibrational state promotes reaction relative to putting the excitation energy in translational motion. Comparisons of vibrational efficacies 
computed with QCT and QD tend to yield mixed results. For reaction of $\mathrm{CH}_{4}$ on metals Mastromatteo and Jackson ${ }^{598}$ and Jiang and $\mathrm{Guo}^{500}$ found larger vibrational efficacies from QD than from QCT calculations, where the dimensionality of the QD and QCT results differed in the latter case. Mastromatteo and Jackson explained this result on the basis of the raw QCT method overestimating the ground state reactivity due to zpe leakage. ${ }^{598}$ On the other hand, Jiang and coworkers note ${ }^{350}$ that $\mathrm{QCT}^{121}$ and $\mathrm{QD}^{120}$ calculations find comparable vibrational efficacies for dissociation of water on $\mathrm{Ni}(111)$. More research is needed into these trends, and also whether perhaps the use of Gaussian weighting can help diminish discrepancies between QCT and QD calculations. Finally, with the present reduced dimensionality models used in the wave packet calculations on DC of $\mathrm{CH}_{4}$ on metals, such as the ones compared to in ref. 500, these wave packet calculations are not expected to yield accurate vibrational efficacies (see Section 4.4 .3 below).

The QCT method is still very much used in studies of diatomic molecules scattering from reactive metal surfaces. Recent studies have addressed reaction of $\mathrm{H}_{2}$ on $\mathrm{Cu}(111),{ }^{43,57,150,152,159,160,531,597}$ $\mathrm{Cu}(100),{ }^{150,172,597} \mathrm{Cu}(211),{ }^{166,511} \mathrm{Ru}(0001),{ }^{150,151} \mathrm{Ni}(111),{ }^{476}$ $\operatorname{Pd}(111),{ }^{158,596,602} \operatorname{Pt}(111),{ }^{150,156,163} \operatorname{Pt}(211),{ }^{48} \mathrm{Au}(111),{ }^{187}$ and $\mathrm{Ag}(111){ }^{58,64,152,186}$ reaction of $\mathrm{N}_{2}$ on $\mathrm{Ru}(0001),{ }^{59,138,139}$ on $\mathrm{W}(110),{ }^{86}$ and on $\mathrm{W}(100),{ }^{132,143}$ reaction of $\mathrm{O}_{2}$ on $\mathrm{Al}(111),{ }^{35}$ $\mathrm{Ag}(111),{ }^{82}$ and $\mathrm{Cu}$ covered $\mathrm{Ru}(0001),{ }^{130}$ and reaction of $\mathrm{HCl}$ on $\mathrm{Au}(111) .{ }^{85,133}$ Additionally, recent QCT studies have addressed vibrationally inelastic scattering of $\mathrm{H}_{2}$ from $\mathrm{Cu}(111)^{57}$ and of $\mathrm{HCl}$ from $\mathrm{Au}(111),{ }^{133}$ rotationally inelastic diffraction of $\mathrm{H}_{2}$ from $\operatorname{NiAl}(110),{ }^{112}$ scattering of $\mathrm{N}_{2}$ from $\mathrm{W}(110)^{109}$ and from $\mathrm{W}(100),{ }^{146}$ and scattering of $\mathrm{O}_{2}$ from $\mathrm{Ag}(111) .{ }^{94}$

The QCT method is also much used in research on reaction of polyatomic molecules on metal surfaces, for systems such as $\mathrm{CH}_{4}+\mathrm{Ni}(111)^{99,137,167,500,598}$ and $\mathrm{Ni}(100),{ }^{598} \mathrm{CHD}_{3}+\mathrm{Ni}(111)^{99,153,161}$ and $\mathrm{Cu}(111),{ }^{63} \mathrm{CH}_{2} \mathrm{D}_{2}+\mathrm{Ni}(111),{ }^{161} \mathrm{CH}_{3} \mathrm{D}+\mathrm{Ni}(111),{ }^{99} \mathrm{CH}_{4}+\operatorname{Ir}(111)$ and $\mathrm{Ir}(332),{ }^{604} \mathrm{D}_{2} \mathrm{O}+\mathrm{Ni}(111),{ }^{121,122,597} \mathrm{CO}_{2}+\mathrm{Ni}(100),{ }^{62,69} \mathrm{CH}_{3} \mathrm{OH}+$ $\mathrm{Cu}(111),{ }^{144}$ and $\mathrm{NH}_{3}+\mathrm{Ru}(0001) .{ }^{66}$

\subsection{On the fly dynamics methods}

The DFMD method can be used to model the DC of a molecule on a mobile metal surface, and to study the influence of $T_{\mathrm{S}}$ on the dissociation (see also Section 4.1.2). A considerable advantage of the method is that the fitting of a high-dimensional PES can be skipped. A disadvantage of the method is that it is computationally expensive to use, with the statistically accurate calculation of sticking or scattering probabilities $<0.01$ currently out of reach. Furthermore, the DFMD method is subject to all the pitfalls of the QCT method, except fitting errors in the PES. An overview of the systems to which the method has been applied has been given in Section 4.1.2.

The effects of ehp excitation and of phonon motion can be taken into account simultaneously with the DFMDEF method (Section 4.1.4). The DFMDEF method has the same advantages and disadvantages as the DFMD method. An overview of the systems to which the method has been applied has been given in Section 4.1.4.

\subsection{QD methods}

Most applications of QD methods to reactive molecule-metal surface scattering use the time-dependent wave packet (TDWP) method $^{605}$ as implemented for molecule-surface scattering. ${ }^{508,509,591}$ In this method, the wave function is expanded in time-independent basis functions, and typically taken in a direct product form of basis functions for different DOFs. The time-dependence is included via time-dependent expansion coefficients. The initial wave function is usually taken as a Gaussian wave packet for motion towards the surface times a wave function describing parallel translational motion, rotational, and vibrational motion. The wave function is propagated in time by acting with the evolution operator on the initial wave function, using a suitable propagation algorithm. State-to-state scattering probabilities can be computed by applying asymptotic boundary conditions in the entrance channel, which involves time-to-energy Fourier transforms. Reaction probabilities can be computed by summing and subtracting the state-to-state scattering probabilities from one, or by computing the reactive flux through a surface positioned in the exit channel. Below, we will discuss applications of this method to diatomic molecules reacting on metals, and to $\mathrm{H}_{2} \mathrm{O}$ and $\mathrm{CH}_{4}$ reacting on metals.

In the time-independent wave packet method ${ }^{606}$ as implemented for molecule-surface reactions ${ }^{607}$ one acts with the Green's function on the initial wave function instead. This method may be viewed as acting with the evolution operator on the initial wave function while simultaneously performing the time-to-energy Fourier transforms required for the asymptotic analysis. Applications of this method are rare, possibly because the propagators are not as efficient as the split-operator method ${ }^{608}$ that can be used in the TDWP method.

In the multi-configuration time-dependent Hartree (MCTDH) method $^{502,609}$ the time-dependence of the wave functions is contained in both the expansion functions and in the basis functions. While this method is in principle highly suitable for studying high-dimensional reactive systems, ${ }^{610,611}$ in practice it has so far only been used to study a few molecule-metal surface reactions. ${ }^{87,505,612-614}$

On the contrary, the RPH method ${ }^{50,203}$ has been used to a far greater extent to study reactive scattering of polyatomic molecules from metal surfaces. This method is similar in spirit to the TDWP method, but propagates the wave function along the reaction coordinate. $^{50,203}$ The wave function is expanded in the local normal mode vibrational states (in vibrationally adiabatic states, taken in the harmonic approximation). Approximations are applied to the asymptotically unbound motions of the polyatomic molecule, i.e., the parallel translational motion and the rotational motion. All vibrations of the molecule can be included for the molecules studied so far $\left(\mathrm{H}_{2} \mathrm{O},{ }^{77} \mathrm{CH}_{4},{ }^{50,203}\right.$ and $\left.\mathrm{CO}_{2}{ }^{71}\right)$. The effect of $T_{\mathrm{S}}$ can be taken into account with the LRS model in a rather accurate, albeit a posteriori fashion (Section 4.1.2). ${ }^{21,30}$ The method is capable of producing semi-quantitative results, and of explaining several of the trends found in experiments. Below we mention and discuss several applications of the RPH method.

Finally, Groß and co-workers have developed a time-independent coupled-channel method that also uses reaction path coordinates, and has been implemented to study the reactive scattering of diatomic molecules from metal surfaces. ${ }^{507}$ 
4.4.1. Wave packet calculations on diatomic molecules interacting with metal surfaces. Systems studied in recent QD calculations on diatomic molecules reacting on metal surfaces while modeling all six molecular DOFs include $\mathrm{H}_{2}+$ $\mathrm{Cu}(111),{ }^{43,102,117,159,169,174,177} \mathrm{Cu}(100),{ }^{46} \mathrm{Cu}(211),{ }^{511} \mathrm{Ag}(111),{ }^{76,80,186}$ $\mathrm{Co}(0001),{ }^{76} \mathrm{Ni}(111),{ }^{476} \mathrm{NiAl}(110),{ }^{112} \mathrm{Pd}(111),{ }^{158} \mathrm{Pt}(111),{ }^{156,163,615}$ $\mathrm{Pt}(211),{ }^{48}$ and $\mathrm{Ru}(0001) .{ }^{116,148,151}$ 6D QD calculations have also been performed on reaction of $\mathrm{HCl}^{106,134}$ and $\mathrm{DCl}^{105}$ on $\mathrm{Au}(111)$, and of $\mathrm{HCl}$ on $\mathrm{Ag}(111),{ }^{65,457,616}$ and on $\mathrm{Ag}(100)$ and $\mathrm{Ag}(110) .{ }^{616,617}$ Finally, 7D QD calculations also considering phonon motion have been performed on sticking of $\mathrm{H}_{2}$ to $\mathrm{Cu}(111) .{ }^{171}$

An important finding from the calculations on diatomic molecules dissociating on metals is that it is usually possible to accurately compute $S_{0}$ by averaging the $S_{0}$ computed with $4 \mathrm{D}$ QD calculations over the impact sites $X$ and $Y$ kept fixed in the $4 \mathrm{D}$ dynamics (the SAED approximation, Section 4.1.1). The 4D calculations model motion in the internal coordinates of the diatomic molecule and of the motion towards the surface. This result was first obtained by Zhang and co-workers for $\mathrm{HCl}+$ $\mathrm{Au}(111),{ }^{106}$ who also found that it was not necessary to make a correction for different zpes in the averaged 4D calculations and the 6D calculations, ${ }^{106}$ as used earlier by Dai and Light in calculations on $\mathrm{H}_{2}+\mathrm{Cu}(111) .{ }^{509}$ The finding that the SAED approximation works well was subsequently confirmed for $\mathrm{HCl}+$ $\mathrm{Ag}(111)$ and $\mathrm{Ag}(100),{ }^{617} \mathrm{H}_{2}+\mathrm{Cu}(111),{ }^{102}$ and for $\mathrm{H}_{2}$ interacting with $\mathrm{Ag}(111)$ and $\mathrm{Co}(0001),{ }^{76}$ though the SAED approximation works less well for $\mathrm{HCl}+\mathrm{Ag}(110) .{ }^{617}$ This approximation and variants of it have been used in several high-dimensional QD calculations on polyatomic molecules reacting with metal surfaces (see below).

4.4.2. Wave packet calculations on $\mathrm{H}_{2} \mathrm{O}$ interacting with metal surfaces. Polyatomic molecule-metal surface systems that have recently received a lot of attention include systems where $\mathrm{H}_{2} \mathrm{O}$ or one of its isotopologues interacts with a metal surface. As shown in Fig. 22 for the example of HOD interacting with rigid $\mathrm{Cu}(111)$, this problem can be characterized by nine DOFs, and full 9D QD calculations have just become possible. ${ }^{61}$

The first QD calculations on a $\mathrm{H}_{2} \mathrm{O}$-metal surface system addressed $\mathrm{H}_{2} \mathrm{O}+\mathrm{Cu}(111) .{ }^{119}$ Copper is a good low temperature water-gas shift catalyst (producing $\mathrm{H}_{2}$ and $\mathrm{CO}_{2}$ from $\mathrm{H}_{2} \mathrm{O}$ and $\mathrm{CO}$ ), while Ni (discussed below) has the disadvantage that it is easily poisoned by carbon following CO dissociation. ${ }^{618}$ The pioneering calculations by Jiang, Guo, and co-workers treated this system with the FSA (in which the PES does not depend on $X$, $Y$, and the azimuthal angle $\alpha$ in Fig. 22). ${ }^{119}$ The 6D calculations considered motion in the remaining six DOFs (see Fig. 22) for impact on the site at which the TS was located (the minimum barrier height is about $1.1 \mathrm{eV}$ as computed ${ }^{119}$ with the PW91 $\mathrm{DF}^{312}$ ). They found that putting energy initially in any of the three vibrations of $\mathrm{H}_{2} \mathrm{O}$ promotes reaction more efficiently than enhancing the $E_{i}$ by the same amount. At roughly the same time Tiwari and co-workers published calculations modeling motion in 3 DOFs explicitly (i.e., $\theta_{2}, Z$, and the distance of the dissociating $\mathrm{H}$-atom to $\mathrm{O}$ ). Their calculations also took into account different projections of the molecule's angular momentum $j$ on the surface normal (i.e., $m_{j}$, which is a conserved quantum number in the flat

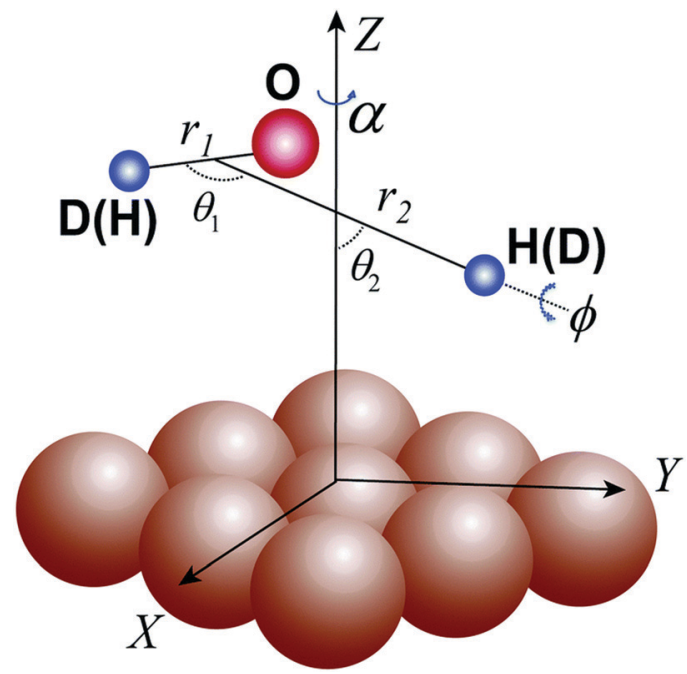

Fig. 22 Coordinate system for $\mathrm{H}_{2} \mathrm{O}$ reacting on a (111) surface of an fcC metal. $Z$ is the distance of the molecule's COM to the surface, and $X$ and $Y$ define its projection on the surface unit cell. $r_{1}$ is the $\mathrm{OH}$ distance of the non-dissociating $\mathrm{OH}$ bond, $r_{2}$ is the distance of the leaving $\mathrm{H}$ atom to the COM of the $\mathrm{OH}$ remaining behind, and $\theta_{1}$ is the angle between the $\boldsymbol{r}_{1}$ and the $\boldsymbol{r}_{2}$ vectors. $\alpha, \theta$, and $\phi$ are the Euler angles defining the orientation of the molecule, where $\alpha$ is the azimuthal angle for the rotation of the molecule about the $Z$-axis, and $\phi$ is the angle for the rotation of the molecule about the body fixed axis taken along $\boldsymbol{r}_{2}$. Reproduced from ref. 103 with permission from the PCCP Owner Societies.

surface approximation) while addressing the effect of the initial rotational state of $\mathrm{H}_{2} \mathrm{O} .{ }^{619}$ Jiang, Guo and coworkers also addressed vibrationally mediated bond selective dissociation of HOD on $\mathrm{Cu}(111)$ with their 6D model, ${ }^{499}$ and very recently their 6D model was used in predictive calculations on vibrationally inelastic scattering of HOD and $\mathrm{H}_{2} \mathrm{O}$ from $\mathrm{Cu}(111) .{ }^{620}$ Furthermore they considered the effect of initially rotationally excited states on dissociation of $\mathrm{H}_{2} \mathrm{O}$ on $\mathrm{Cu}(111)$ with $6+1 \mathrm{D}$ calculations, ${ }^{108}$ which were performed for different (conserved) $M$ states for $J>0$, where $M$ is the projection of the rotational angular momentum quantum number $J$ of $\mathrm{H}_{2} \mathrm{O}$ on the surface.

The $\mathrm{H}_{2} \mathrm{O}+\mathrm{Cu}(111)$ system was next studied by Zhang and co-workers in $7 \mathrm{D}$ calculations performed for different fixed sites. ${ }^{100}$ These calculations also considered the dependence of the molecule-surface interaction on $\alpha$ (Fig. 22). Zhang and coworkers found this dependence to be quite strong on most impact sites. ${ }^{100}$ In particular, they showed that for quantitative accuracy the use of a 6D model (where the calculation is either performed for $\alpha$ corresponding to the global TS, or the value corresponding to the minimum barrier for the specific site considered) does not suffice. However, quite accurate results could be obtained for the rovibrational ground state of $\mathrm{H}_{2} \mathrm{O}$ by performing 6D calculations for 18 equally spaced values of $\alpha$, and averaging the resulting reaction probabilities (the azimuthal averaging (AA) approximation). ${ }^{100}$ Subsequent work showed that this also holds for initially vibrationally excited states of $\mathrm{H}_{2} \mathrm{O}$. ${ }^{621}$

This work set the stage for the first full-dimensional (9D) QD calculations on $\mathrm{H}_{2} \mathrm{O}$ dissociating on a static metal surface, which were also performed for the $\mathrm{Cu}(111)$ surface. ${ }^{61}$ This work 


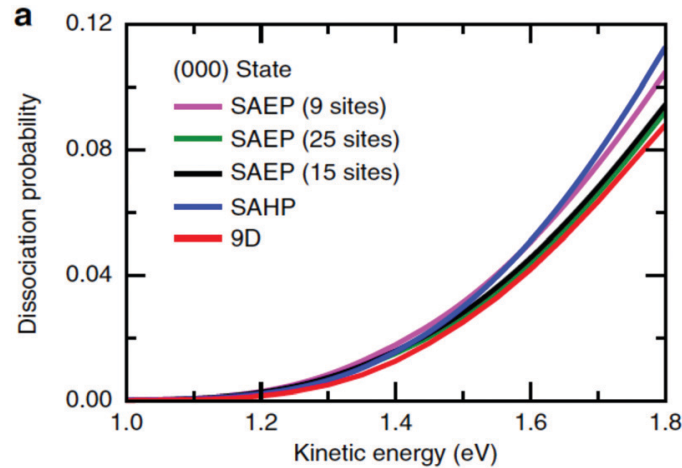

Fig. $23 \mathrm{~S}_{0}$ computed $^{61}$ in 9D QD calculations for $\mathrm{H}_{2} \mathrm{O}$ in its rovibrational ground state colliding with $\mathrm{Cu}(111)$ are compared with values computed using different sudden approximations for molecular motion along the surface, i.e., using the SAED approximation employing 9 and 25 sites (called SAEP here), using the SAED approximation employing 15 sites in half the irreducible triangle of the surface unit cell and assuming symmetry (called SAEP 15 sites here), and using the SAHP approximation. Reprinted from Z. J. Zhang, T. H. Liu, B. N. Fu, X. M. Yang and D. H. Zhang, First-principles quantum dynamical theory for the dissociative chemisorption of $\mathrm{H}_{2} \mathrm{O}$ on rigid Cu(111), Nat. Commun., 2016, 7, 11953, licensed under CC-BY 4.0.

also showed that accurate results can be obtained for reaction probabilities $\geq 0.001$ using the SAED approximation, by performing explicit 7D QD calculations for 25 sites and averaging the computed reaction probabilities (called "SAEP" in Fig. 23) ${ }^{61}$ Accurate results could also be obtained using 15 sites contained in half the irreducible triangle of the surface unit cell, amounting to the approximation that the $\mathrm{H}_{2} \mathrm{O}$ interaction with the fcc site equals that with the hcp site ("SAEP (15 sites)" in Fig. 23). In contrast, the approximation of shifting the reaction probability curve computed for the TS by an energy difference obtained from a harmonic approximation to the change of the site-dependent $E_{\mathrm{b}}$ with $X$ and $Y$, which was introduced to model reaction of $\mathrm{CH}_{4}$ with metals in the RPH method, ${ }^{50}$ was found not to work well for $\mathrm{H}_{2} \mathrm{O}+\mathrm{Cu}(111)$. Results obtained with this approximation (abbreviated "SAHP" for "site-averaging with harmonic potential" approximation) are also shown in Fig. 23. In the SAHP approximation, a reaction probability curve is computed with dynamics for just one site (usually the TS site or a site close to it), and for the other sites this reaction probability curve is shifted with the difference in $E_{\mathrm{b}}$ between the sites, after which averaging is performed. The difference between the SAHP and SAED results shows that it is not just $E_{\mathrm{b}}$ that determines the reactivity at a given site. Instead, the full topology of the PES at the site (e.g. the tightness of the reduced dimensionality TS) needs to be taken into account, as this strongly affects the energy dependence of the $S_{0}$ at the site. ${ }^{61}$ This work set a standard for accurate QD calculations on $\mathrm{H}_{2} \mathrm{O}$ dissociating on metals: ${ }^{61}$ such calculations should either be full-dimensional (9D), or they should use the SAED approximation averaging over 7D QD calculations performed for enough fixed surface sites. Using the SAED approximation, Zhang and co-workers also published results for $\mathrm{HOD}^{103}$ and $\mathrm{D}_{2} \mathrm{O}^{622}$ DC on $\mathrm{Cu}(111)$.

The first water-metal system for which supersonic $\mathrm{MB}$ experiments were performed is the $\mathrm{D}_{2} \mathrm{O}+\mathrm{Ni}(111)$ system, with $\mathrm{D}_{2} \mathrm{O}$ chosen because the lasers owned by the experimentalists could be used to pre-excite its anti-symmetric stretch vibration. ${ }^{78}$ This system has a minimum $E_{\mathrm{b}}$ of about $0.77 \mathrm{eV}$ as computed ${ }^{78}$ with the PW91 DF. ${ }^{312}$ The experimental results were published along with QD calculations on the basis of a PW91 PES and a semiempirically corrected PES, and the latter gave a semi-quantitative description of the experimental results. ${ }^{78}$ In this pioneering work, a $6 \mathrm{D}$ QD calculation was done for reaction at the TS values of $X, Y$, and $\alpha$ (see Fig. 22). Next, the SAHP approach was used to average the computed reaction probability curve over $X$ and $Y$, computing the barriers for the $\alpha$-value of the TS. The work of Jiang and Huo went beyond the later work of Zhang and coworkers ${ }^{62}$ in one important aspect: the impact of $T_{\mathrm{S}}$ and surface atom motion was addressed with the LRS model ${ }^{524,525}$ discussed in Section 4.1.2. However, hindsight suggests the use of the SAHP approach and of a single value of $\alpha$ to be severe approximations.

Jiang and Guo next investigated the influence of the SAHP and SAED approaches on the sticking of $\mathrm{D}_{2} \mathrm{O}$ to $\mathrm{Ni}(111)$ by averaging 6D QD calculations (performed for one value of $\alpha$ ) and 7D QCT calculations (also modeling the dynamics in $\alpha$ ). ${ }^{623}$ Their work confirmed that for obtaining accurate results for water-metal systems the SAED method needs to be used. ${ }^{623}$ They also tested an approach in which the same energy dependence of site-dependent reaction probability curves was assumed as for the TS, but the difference in the site-dependent $E_{\mathrm{b}}$ was explicitly computed with DFT rather than obtained using a harmonic expansion around the TS. This approach (which they called "site averaging by explicit energy shifting", which may be abbreviated as "SAEES") gave results that hardly differed from the SAHP results. The authors concluded that it is incorrect to assume the same energy dependence of the $S_{0}$ at all surface sites, as their shape is controlled by the detailed topology of the PES when calculated with dynamics, and not just by the $E_{\mathrm{b}} \cdot{ }^{623}$ In contrast to results discussed in Section 4.2 (see Fig. 20), they found the results of 9D QCT calculations to be in rather good agreement with the site-averaged 6D QD results obtained with the SAED approach. ${ }^{623}$ However, the likely cause of this is that the QD $S_{0}$ were obtained by averaging over $S_{0}$ obtained for the $\alpha$-value most favorable to reaction, while the QCT calculations also took into account the dynamics at $\alpha$-values that are unfavorable to reaction. This underlines the importance of the use of the same dynamical model when assessing the quality of the QCT approach (see also Section 4.2).

Jiang and Guo and co-workers next introduced motion in $\alpha$ in their QD model, and obtained approximate 9D results for $\mathrm{D}_{2} \mathrm{O}+\mathrm{Ni}(111)$ with the SAED approach using a PW91 PES. ${ }^{120}$ Their work showed the convergence of approximate 9D $(7+2 \mathrm{D}$ SAED) results with the number of sites averaged over, as previously found for $\mathrm{H}_{2} \mathrm{O}+\mathrm{Cu}(111) .{ }^{61}$ However, they did not yet test whether the converged SAED results compare well with 9D QD results for $\mathrm{D}_{2} \mathrm{O}+\mathrm{Ni}(111)$. 9D QD calculations for $\mathrm{D}_{2} \mathrm{O}+$ $\mathrm{Ni}(111)$ should be harder to perform than for $\mathrm{H}_{2} \mathrm{O}+\mathrm{Cu}(111)$, as modeling the QD of $\mathrm{D}_{2} \mathrm{O}$ rather than $\mathrm{H}_{2} \mathrm{O}$ should require a larger number of basis functions. Their work ${ }^{120}$ also confirmed the validity of the AA approximation found earlier for $\mathrm{H}_{2} \mathrm{O}+$ $\mathrm{Cu}(111)^{100}$ for $\mathrm{D}_{2} \mathrm{O}+\mathrm{Ni}(111)$, by averaging $6 \mathrm{D}$ results of calculations performed for a large number of angles $\alpha$ to obtain approximate 7D 


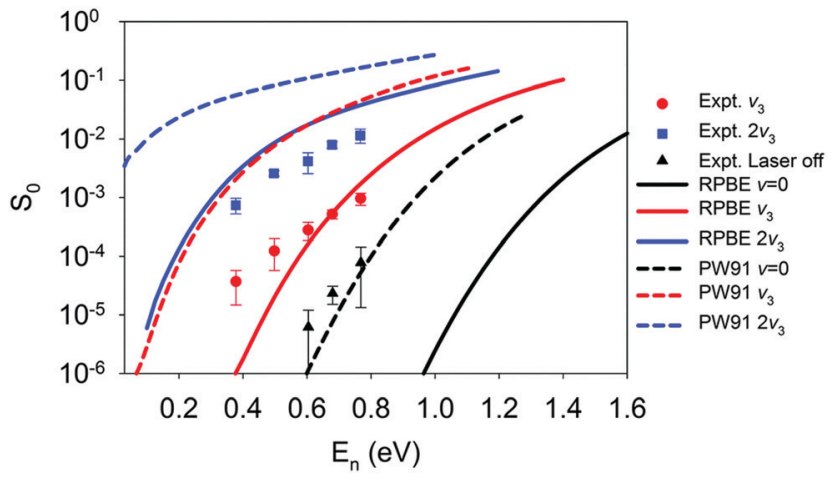

Fig. $24 S_{0}$ measured $^{78}$ for $D_{2} \mathrm{O}+\mathrm{Ni}(111)$ are compared to $Q D S_{0}$ computed $^{155}$ by site-averaging 7D results and correcting for surface temperature effects using the LRS model, using a PW91 PES (dashed lines) and using an RPBE PES (solid lines), for the three initial vibrational states indicated. Reproduced from ref. 155 with permission from the PCCP Owner Societies.

QD results, and comparing these to exact 7D results. Jiang and Guo $^{155}$ also obtained approximate 9D QD results for a thermal surface based on site-averaged 7D QD calculations, the SAED approach, and the LRS model using a PES obtained with the $\mathrm{RPBE}^{196} \mathrm{DF}$. The RPBE $S_{0}$ they computed were in reasonable agreement with the experimental values for the $1 \nu_{3}$ and $2 \nu_{3}$ states (see Fig. 24). The implications of the differences with the PW91 results $^{120}$ (see also Fig. 24) will be discussed below.

Jiang considered the effect of the initial rotational state of $\mathrm{D}_{2} \mathrm{O}$ on sticking on $\mathrm{Ni}(111)$ with approximate $9 \mathrm{D}$ calculations. ${ }^{131}$ The topic addressed is important as the original experiment ${ }^{78}$ modeled by Jiang pre-excited $\mathrm{D}_{2} \mathrm{O}$ to specific $J_{K_{\mathrm{a}} K_{\mathrm{c}}}$ rotational states $\left(2_{12}\right.$ for $1 \nu_{3}$ and $3_{13}$ for $2 \nu_{3}$ ). Jiang ${ }^{131}$ found that the approximate 9D results hardly differed from the approximate $9 \mathrm{D}$ results for the $\left(0_{00}, 1 \nu_{3}\right)$ and $\left(0_{00}, 2 \nu_{3}\right)$ states when also averaging over the magnetic rotational quantum number. Therefor the differences between the approximate 9D results and experiment in Fig. 24 are not related to an incorrect modeling of the initial rotational state of $\mathrm{D}_{2} \mathrm{O} \cdot{ }^{131}$ According to Jiang steric effects may be observable in an experiment using polarized laser light to pre-excite the $1 \nu_{3}$ or $2 \nu_{3}$ states, as siteaveraged results do depend on the initial rotational state if the magnetic rotational quantum number is not averaged over. ${ }^{131}$

Zhang and co-workers ${ }^{98}$ also obtained full 9D QD results for $\mathrm{H}_{2} \mathrm{O}+\mathrm{Ni}(100)$, based on a PW91 PES. Their PW91 PES has a TS near the bridge site, with a barrier of only $0.31 \mathrm{eV},{ }^{98}$ which is much lower than obtained before for $\mathrm{H}_{2} \mathrm{O}+\mathrm{Ni}(111)(0.77 \mathrm{eV}) .^{78}$ Their QD results for $\mathrm{H}_{2} \mathrm{O}+\mathrm{Ni}(100)$ again confirmed the validity of the SAED approach, and showed that SAHP results are more approximate. ${ }^{98}$

Finally, a word of caution is in order concerning the accuracy of the QD calculations on DC of $\mathrm{H}_{2} \mathrm{O}$ on metals. The high dimensionality of the calculations by itself does not guarantee their accuracy. The 7D and 9D QD calculations typically use the split-operator method ${ }^{608}$ to propagate the wave function in time, with a fairly large time step (10 atomic units of time ${ }^{61,120}$ ). Results of convergence tests have been shown for the number of points used for motion in $X$ and $Y$ and for the rotational basis set, ${ }^{61}$ but no such tests have been shown for the number of vibrational basis functions used for $r_{1}$ and $r_{2}$ (see Fig. 22), and convergence tests have not been shown for $S_{0}$ lower than $10^{-3}$. The calculations use optical potentials defined over small ranges of $r$ and $Z,{ }^{61,120}$ and the parameters of these potentials have to be chosen carefully to avoid reflection of the wave function. ${ }^{624}$ Flux analysis to obtain reaction probabilities is performed at values of $r_{2}$ such that the minimum energy path for a given site has not yet decayed to the asymptotic energy (see for instance Fig. $2 \mathrm{~b}$ of ref. 155). Papers presenting high-dimensional QD results may present reaction probabilities as low as $10^{-561}$ or even $10^{-7,155}$ but it is not clear how well converged such results really are.

4.4.3. Wave packet calculations on $\mathrm{CH}_{4}$ interacting with metal surfaces. Another class of polyatomic molecule-metal surface reactions that have recently received a lot of attention in high-dimensional QD calculations consists of systems where $\mathrm{CH}_{4}$ interacts with a metal surface. The DOFs that have been treated in such calculations (excepting calculations with the RPH method to be discussed below) are visualized in Fig. 25. Motion in the DOFs shown can be modeled with a quantum Hamiltonian that is based on a dynamical model proposed by Palma and Clary ${ }^{625}$ but extended for gas-surface reactions. ${ }^{60,145}$ In the dynamical model implicit in Fig. 25, the $C_{3 \mathrm{v}}$ symmetry of the methyl fragment is preserved. The internal coordinates shown for $\mathrm{CH}_{4}$ are $r, s, \chi, \theta_{2}$, and $\varphi_{2}$, while the coordinates

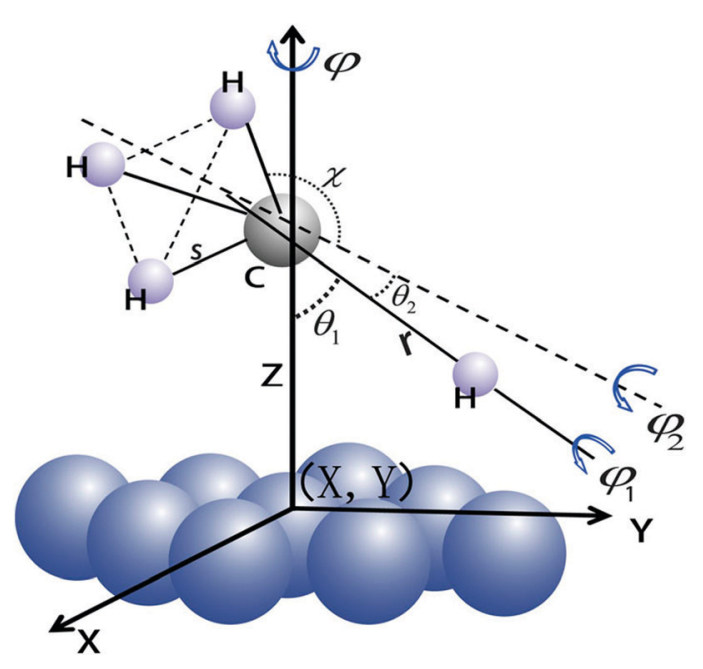

Fig. 25 Coordinate system for $\mathrm{CH}_{4}$ reacting on a (111) surface of an fcc metal, where the remaining $\mathrm{CH}_{3}$ moiety is restricted to have $C_{3 v}$ symmetry. $Z$ is the distance of the molecule's COM to the surface, and $X$ and $Y$ define its projection on the surface unit cell. $s$ is the $\mathrm{CH}$ distance in the methyl fragment, $r$ is the distance of the leaving $\mathrm{H}$ atom to the $\mathrm{COM}$ of the $\mathrm{CH}_{3}$ remaining behind, and $\chi$ is the umbrella angle of the $\mathrm{CH}_{3}$ fragment relative to its $C_{3 v}$ symmetry axis. $\varphi, \theta_{1}$, and $\varphi_{1}$ are the Euler angles defining the orientation of the molecule, where $\varphi$ is the azimuthal angle for the rotation of the molecule about the $Z$-axis, and $\varphi_{1}$ is the angle for the rotation of the molecule about the body fixed axis taken along $\boldsymbol{r}$. Furthermore, $\theta_{2}$, and $\varphi_{2}$ are the polar and azimuthal angles of orientation of the methyl fragment with respect to $r$. Reprinted from [X. J. Shen, Z. J. Zhang and D. H. Zhang, Methane dissociation on $\mathrm{Ni}(111)$ : A seven-dimensional to nine-dimensional quantum dynamics study. J. Chem. Phys., 2017, 147, 024702], with the permission of AIP Publishing. 
describing translational motion and rotation are $X, Y$, and $Z$, and $\theta_{1}, \varphi_{1}$, and $\varphi$, respectively.

The first QD calculations that treated more than two molecular DOFs modeled motion in $Z, r$, and $\theta_{1}{ }^{626}$ and were therefore $3 \mathrm{D}$ calculations, addressing dissociation of $\mathrm{CH}_{4}$ on $\mathrm{Ni}(111)$. Modeling reaction of $\mathrm{CH}_{4}$ on Ni(100), Carré and Jackson ${ }^{627}$ also considered the effect of the magnetic rotational quantum number $M$ using the FSA in which $M$ is conserved, performing $3+1 \mathrm{D}$ calculations. They also treated the effect of surface atom motion with the SM model. ${ }^{516}$ Xiang et al. added motion in $\varphi_{1}$, performing $4+1 \mathrm{D}$ calculations already considering motion in all rotational molecular DOFs of $\mathrm{CH}_{4}$ reacting on $\mathrm{Cu}(111)$, albeit that the approximation was made that $M$ was conserved. ${ }^{628}$ Next, Jackson and coworkers also performed $4+1 \mathrm{D}$ QD calculations on $\mathrm{CH}_{4}$ reacting on $\mathrm{Ni}(111)^{517,524,525,629,630}$ and $\mathrm{Pt}(111),{ }^{524,630}$ but modeling motion in the "phonon" coordinate $Q$, representing the motion of the top layer surface atom above which $\mathrm{CH}_{4}$ dissociates, instead of in $\varphi_{1}$ (see Fig. 25). Their calculations showed the large effect $T_{\mathrm{s}}$ can have on the DC of $\mathrm{CH}_{4}$, and established the validity of the LRS model for taking into account the effects of $T_{\mathrm{s}}$ a posteriori. ${ }^{524}$ Füchsel et al. performed $5+1 \mathrm{D}$ QD calculations on $\mathrm{CH}_{4}$ reacting on $\mathrm{Pt}(111)$, modeling motion in $Z, r$, all rotational DOFs, and treating motion in $\theta_{2}$ adiabatically. ${ }^{87}$ Their calculations yielded insight in the rotational dynamics of the reaction, showing that neither the rotational sudden approximation (RSA) nor the RAA is accurate for the DC of $\mathrm{CH}_{4}$ in this system.

The first QD calculations explicitly treating motion in as much as eight DOFs were done by Jiang and Guo and coworkers. ${ }^{60}$ Their calculations explicitly treated motion in $r, s, \chi, \theta_{2}, \varphi_{2}, Z, \theta_{1}$, and $\varphi_{1}$, and addressed dissociation of $\mathrm{CH}_{4}$ on $\mathrm{Ni}(111)$. A 12D PES was used, which was calculated in the FSA for the values of $X, Y$ and $\varphi$ corresponding to the TS. With the way the $\mathrm{CH}_{4}$ was modeled, dissociation was possible in only one $\mathrm{CH}$-bond, and therefore the computed $S_{0}$ were multiplied with a factor four. The authors also noted restrictions following from the revised form ${ }^{631}$ of the Palma-Clary Hamiltonian: ${ }^{625}$ due to the approximations made to the methyl fragment, only two stretches and three bend modes are modeled, instead of 4 and 5, respectively. Also, the use of the model leads to a large error in the frequency of the $v_{2}$ bending mode, ${ }^{60}$ as this mode does not retain the $C_{3 \mathrm{v}}$ symmetry of the non-reacting umbrella. ${ }^{60}$ The pioneering calculation of Jiang and Guo and coworkers ${ }^{60}$ already took into account the effects of motion along the surface and of surface atom motion in an approximate way, through the SAHP approximation and the LRS model, respectively, as had been done earlier in RPH calculations by Jackson and Nave. ${ }^{50}$ The calculations provided semi-quantitative agreement with experiments on the laser-off reactivity, and on the reactivity of the $1 v_{3}, 2 v_{3}$, and $3 v_{4}$ states.

Next Zhang and co-workers computed a 15D PES for $\mathrm{CH}_{4}+$ $\mathrm{Ni}(111)$ and used this in 7D QD calculations. ${ }^{456}$ Compared to the earlier work of Jiang and Guo and co-workers, ${ }^{60}$ differences were that $s$ was kept frozen, but the authors did perform their explicit dynamics calculations for an additional impact site (the fcc site) and for additional values of the angle $\varphi .{ }^{456} \mathrm{They}^{456}$ used the same approximations (SAHP and LRS) as Jiang, Guo, and co-workers. ${ }^{60}$ An important difference with the work of the latter $^{60}$ is that, even though only one of the $\mathrm{CH}$-bonds can dissociate in their model, Zhang and co-workers ${ }^{456}$ did not multiply their computed $S_{0}$ with a factor 4 . The reason was that if they would do that their computed reaction probabilities would exceed 1 at high $E_{i}{ }^{529}$ Zhang and co-workers also omitted the factor 4 in their later work, ${ }^{73,123,145,528}$ and mentioned that future research should explore whether multiplication with this symmetry factor should be performed in full-dimensional QD calculations. ${ }^{456}$ Whether or not the multiplication with the symmetry factor of 4 can be omitted currently represents a major uncertainty in wave packet calculations on DC of $\mathrm{CH}_{4}$ on metal surfaces. ${ }^{456}$

Zhang and co-workers also used their 15D PES for $\mathrm{CH}_{4}+$ $\mathrm{Ni}(111)$ to test a different model (the VTSR model) for taking into account the effect of surface phonons and $T_{\mathrm{s}}^{528}$ (see Section 4.1.2).

Zhang and co-workers performed the first 9D QD simulations on $\mathrm{CH}_{4}$ reacting on a metal surface ( $\left.\mathrm{Ni}(111)\right)$, and used these to test several approximations. ${ }^{123,145}$ In the 9D calculations, they modeled motion in the 8 DOFs also used by Jiang and Guo and co-workers, ${ }^{60}$ and in addition modeled motion in $\varphi$. One approximation tested is one in which the calculations are performed for a single value of $\varphi$ (i.e., 8D calculations). Calculations performed for a single value of $\varphi$ (i.e., the TS value, dashed blue and black lines in Fig. 26) tend to overestimate $S_{0}$ as computed with $\varphi$ as an additional DOF (blue and black solid lines in Fig. 26). ${ }^{123}$ This is true for calculations modeling impact on a single site (blue lines in Fig. 26), but also for calculations that are site averaged using the SAHP approximation (SAHP, black lines in Fig. 26). ${ }^{123}$ The effect is that the 9D calculations also sample orientations in

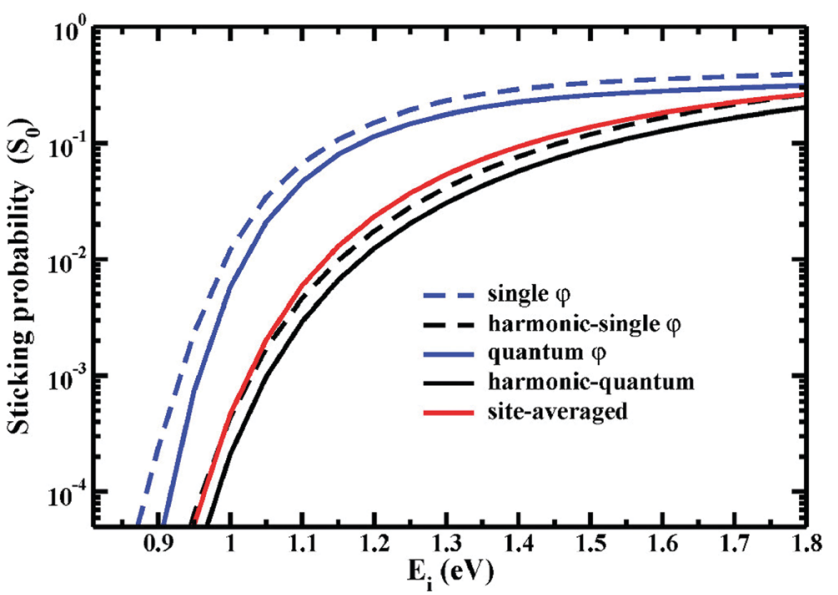

Fig. $26 S_{0}$ computed ${ }^{123}$ with 8D (dashed lines) and 9D (solid lines) QD calculations on $\mathrm{CH}_{4}+\mathrm{Ni}(111)$ are compared. In the 8D calculations, only a single value for $\varphi$ was used, while in the 9D calculations it is treated as a DOF. The curves indicated by blue lines model impact on a single site, while the curves indicated by the black lines were obtained using site averaging of the $8 \mathrm{D}$ and $9 \mathrm{D}$ results with the SAHP method. The red curve was obtained by site averaging 9D results with the SAEEP method. Reprinted from [X. J. Shen, Z. J. Zhang and D. H. Zhang, Communication: methane dissociation on $\mathrm{Ni}(111)$ surface: importance of azimuth and surface impact site, J. Chem. Phys., 2016, 144, 101101], with the permission of AIP Publishing. 
which $\varphi$ is more unfavorable to reaction. ${ }^{123}$ While Fig. 26 shows that taking $\varphi$ into account is important for $\mathrm{DC}$ of $\mathrm{CH}_{4}$ on $\mathrm{Ni}(111)$, it is not as important as it is for $\mathrm{H}_{2} \mathrm{O}+\mathrm{Cu}(111)$ or $\mathrm{Ni}(111)$ (see e.g. Fig. 5 of ref. 621 and Fig. 3 of ref. 120, respectively). Moreover, higher $S_{0}$ were obtained if the site-averaging was performed with the SAEES method (red curve in Fig. 26) than with the SAHP method (black solid curve in Fig. 26). ${ }^{123}$ The reason is that the harmonic expansion in the SAHP method overestimates the barriers far away from the impact point corresponding to the TS. ${ }^{89,123}$

In $8 \mathrm{D}$ calculations on $\mathrm{CH}_{4}+\mathrm{Ni}(111)$ the approximation of freezing the symmetric stretch coordinate of the inert umbrella $\left(s\right.$ in Fig. 25) turned out to work much better ${ }^{123}$ than the approximation of freezing $\varphi$ (see also Fig. 25). ${ }^{145}$ Finally, Zhang and co-workers determined that determining the $X, Y$-dependent barrier from a linear relationship between this barrier height and the distance from the top site yields better results than the use of the SAHP approximation. ${ }^{145}$ Energy shifting approximations for averaging over $X$ and $Y$ should work better for $\mathrm{CH}_{4}$-metal than for $\mathrm{H}_{2} \mathrm{O}$-metal surface systems, for which the shape of the sitedependent reaction probability curve computed with explicit dynamics may strongly depend on $X$ and $Y$ (compare Fig. 4 of ref. 120 for $\mathrm{D}_{2} \mathrm{O}+\mathrm{Ni}(111)$ to Fig. 3a of ref. 123 for $\left.\mathrm{CH}_{4}+\mathrm{Ni}(111)\right)$.

Finally, Zhang and co-workers performed $7+1 \mathrm{D}$ QD calculations on $\mathrm{CH}_{4}$ and $\mathrm{CD}_{4}$ reacting on $\mathrm{Ni}(100)$, on the basis of a $15 \mathrm{D}$ PES $^{73}$ computed with the PBE DF. ${ }^{195}$ They modeled motion in the same DOFs as Jiang and Guo and co-workers, ${ }^{60}$ except that $s$ was frozen. However, the calculations were done using the AA approximation, i.e., 7D QD calculations were performed for several (12) values of $\varphi$, and subsequently averaged. Additional averaging was performed over 15 points in $X$ and $Y$, most likely with the SAED approach. The VTSR model ${ }^{528}$ (Section 4.1.2) was used to include the effect of $T_{\mathrm{s}}$. The $S_{0}$ computed for the ground vibrational state of $\mathrm{CH}_{4}$ were in good agreement with experimental laser-off results obtained for $T_{\mathrm{N}}=400 \mathrm{~K}^{201}$ and $375-425 \mathrm{~K},{ }^{202}$ and $T_{\mathrm{S}}=475 \mathrm{~K}^{201,202}$ (see Fig. 2). ${ }^{73}$ While this agreement is encouraging, it is unclear what the agreement achieved says about the accuracy of the QD model, in the light of the uncertainty regarding the multiplication with the symmetry factor 4 (which was not performed ${ }^{73}$ ), the symmetry constraints on the inert umbrella (which also imply that the zpe correction to the barrier could be different), the fact that $T_{\mathrm{N}}$ was ignored in the calculations comparing with laser-off experiments, and the use of a standard GGA DF.

While consequences of the symmetry constraints on the inert umbrella were not addressed in surface reaction dynamics calculations, they were addressed for the gas phase $\mathrm{CH}_{4}+\mathrm{H}$ reaction. ${ }^{632}$ Initial-state selected reaction probabilities for total angular momentum $J=0$ and initial rotational angular momentum of methane $j=0$ are shown in Fig. 27 for the vibrational ground state and the initial bend-excited states $\nu_{2}=1$ and $\nu_{4}=1 .^{632}$ The results were computed treating all 12 DOFs, as well as using two 8D ("crude" and "adiabatic", with the latter being similar to the Palma and Clary Hamiltonian ${ }^{625}$ used in surface reaction dynamics calculations $^{60,73,123,145,456,528}$ ) models in which the $C_{3 \mathrm{v}}$ symmetry of the remaining methyl fragment was preserved. As can be seen, the $8 \mathrm{D}$ adiabatic reaction probabilities are in reasonable agreement with the exact $12 \mathrm{D}$ results for the rovibrational ground state,

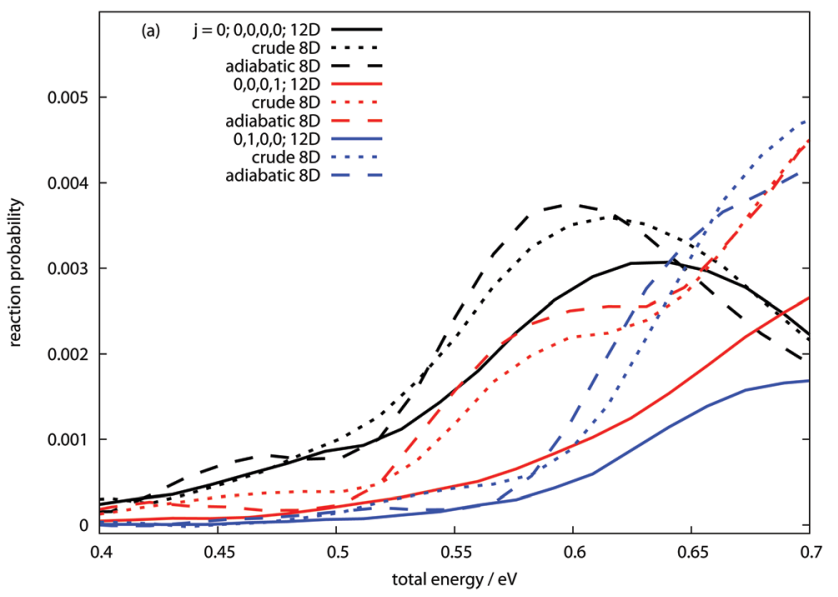

Fig. 27 Initial state resolved reaction probabilities obtained ${ }^{632}$ for the $\mathrm{H}+$ $\mathrm{CH}_{4} \rightarrow \mathrm{H}_{2}+\mathrm{CH}_{3}$ reaction are compared for full-dimensional (12D) QD calculations and for two types of reduced dimensionality calculations in which the $C_{3 v}$ symmetry of the methyl fragment is treated as conserved (crude 8D and adiabatic 8D). The calculations were done for the initial vibrational ground state (0000), the $\nu_{2}=1$ vibrationally excited state (0100), and the $\nu_{4}=1$ vibrationally excited state. In all cases the total angular momentum $\mathrm{J}$ and the initial angular momentum of $\mathrm{CH}_{4}, j$, are 0 . Reprinted from [R. Welsch and U. Manthe, Full-dimensional and reduced-dimensional calculations of initial state-selected reaction probabilities studying the $\mathrm{H}+\mathrm{CH}_{4} \rightarrow \mathrm{H}_{2}+\mathrm{CH}_{3}$ reaction on a neural network PES, J. Chem. Phys., 2015, 142, 064309], with the permission of AIP Publishing.

but note that for the total energy of $0.56 \mathrm{eV}$ the adiabatic $8 \mathrm{D}$ result exceeds the $12 \mathrm{D}$ result by about $50 \% .^{632}$

More importantly, the $8 \mathrm{D}$ adiabatic reaction probabilities for the $\nu_{2}=1$ and $\nu_{4}=1$ substantially overestimate the $12 \mathrm{D}$ reaction probabilities (Fig. 27). ${ }^{632}$ As discussed by Welsh and Manthe, these results should not come as a surprise, as the $8 \mathrm{D} \nu_{4}=1$ state lacks the threefold degeneracy this state has in full dimensionality. Additionally the $8 \mathrm{D} \nu_{2}=1$ state erroneously contains components of both the $f_{2}$-symmetric umbrella and the $e$-symmetric bending vibrations of methane (it should be $e$-symmetric). So while it is true that the $8 \mathrm{D}$ Palma and Clary model "includes representatives of all $\mathrm{CH}_{4}$ vibrational modes", 60 the work of Welsch and Manthe suggests that this does not suffice for the accurate prediction of initial-state selected reaction probabilities. ${ }^{632}$ Their work suggested similarly large problems for the $\nu_{1}=1$ and $\nu_{3}=1$ states, ${ }^{632}$ although this is less certain as later work suggested that the $12 \mathrm{D}$ results for these states were not yet converged with respect to the single particle functions basis ${ }^{633}$ (the $8 \mathrm{D}$ calculations did use the same amounts of spfs, ${ }^{632}$ suggesting that the problem noted is real for the stretch excited states as well). A saving grace for the computation of laser-off reaction probabilities may be that reaction probabilities averaged over the $\nu_{1}=1$ and $\nu_{3}=1$ states on the one hand, and reaction probabilities averaged over the $\nu_{2}=1$ and $\nu_{4}=1$ states on the other hand, were in much better agreement with the corresponding 12 D results (see Fig. 10 of ref. 632, but note once again the $50 \%$ deviation observed for the ground vibrational state at the total energy of $0.55 \mathrm{eV}$ in Fig. 27, which remains). In summary, while QD calculations restricting the methyl fragment to $C_{3 \mathrm{v}}$ symmetry of course do take quantum effects into account, 
the gas phase comparison discussed above suggests that the approach should not be used to compute initial-state selected reaction probabilities for excited vibrational states. Semiquantitative accuracy may however be achieved if the reaction is dominated by the ground vibrational state, and for laser-off reaction.

4.4.4. RPH calculations on polyatomic molecules interacting with metal surfaces. QD calculations using the RPH have been performed for $\mathrm{CH}_{4}+\mathrm{Ni}(100),{ }^{50,79,203,598} \mathrm{CH}_{4}+\mathrm{Ni}(111)^{21,79,81,89,598}$ $\mathrm{CH}_{4}+\mathrm{Ni}(211),{ }^{178,179} \mathrm{CH}_{4}+\operatorname{Pt}(110)-(1 \times 2),{ }^{79} \mathrm{CH}_{4}+\mathrm{Pt}(111),{ }^{527}$ $\mathrm{CH}_{4}+\mathrm{Pt}(211),{ }^{162} \mathrm{CH}_{4}+\operatorname{Ir}(111),{ }^{634} \mathrm{H}_{2} \mathrm{O}+\mathrm{Ni}(111),{ }^{72,77} \mathrm{H}_{2} \mathrm{O}+$ $\mathrm{Ni}(100),{ }^{72} \mathrm{H}_{2} \mathrm{O}+\mathrm{Ni}(110),{ }^{72}$ and $\mathrm{CO}_{2}+\mathrm{Ni}(100) .{ }^{71}$

The most important approximations made in the QD RPH method concern the molecule's rotations and parallel translational motion. In the original applications of the method the RAA was made to the rotations of $\mathrm{CH}_{4} \cdot{ }^{50,79,81} \mathrm{~A}$ subsequent comparative study of QD RPH and AIMD results for $\mathrm{CH}_{4}+\mathrm{Ni}(111)$ and $\mathrm{Pt}(111)$ suggested that a better (although also imperfect) approximation to the rotational reaction dynamics would be to make a RSA. ${ }^{89}$ In this approximation, reaction probabilities computed with the conventional RPH method are corrected by also computing reaction probabilities for angles $\theta$ of the reactive $\mathrm{CH}$ bond with the surface normal not equal to $\theta^{\mathrm{TS}}$. Around the TS $E_{\mathrm{b}}$ is assumed to vary with $\theta$ according to

$$
\Delta V=\frac{1}{2} k_{\theta}\left(\theta-\theta^{\mathrm{TS}}\right)^{2}
$$

where $\Delta V$ is the change in $E_{\mathrm{b}}$. Reaction probabilities in the implementation of the RSA used are then averaged over $\theta$, assuming the reaction probability curve to equal the curve computed for $\theta=\theta^{\mathrm{TS}}$, but shifted upwards in energy with $\Delta V$. As Fig. 28 shows the computed $S_{0}$ can be rather sensitive to

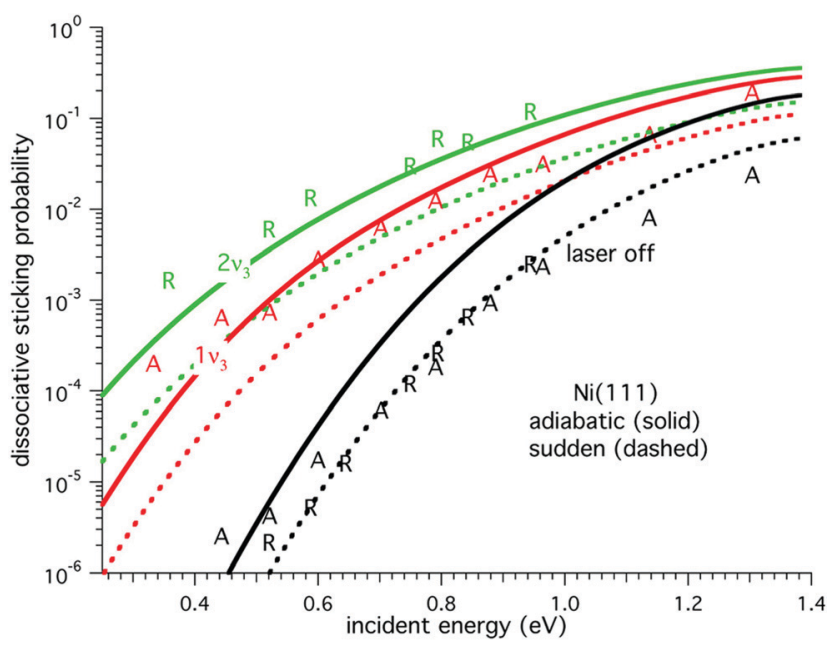

Fig. $28 \mathrm{~S}_{0}$ computed $^{89}$ with the $\mathrm{RPH}$ method for $\mathrm{CH}_{4}+\mathrm{Ni}(111)$ at $T_{\mathrm{s}}=475 \mathrm{~K}$ are shown as a function of incidence energy, using the rotationally adiabatic approximation (solid lines) and the rotational sudden approximation (dashed lines). Results are shown for three initial vibrational states. Also shown are experimental results (" $A$ " symbols from ref. 14, " $R$ " symbols from ref. 635). Reprinted from [B. Jackson, F. Nattino and G. $\mathrm{J}$. Kroes, Dissociative chemisorption of methane on metal surfaces: tests of dynamical assumptions using quantum models and ab initio molecular dynamics, J. Chem. Phys., 2014, 141, 054102], with the permission of AIP Publishing. whether the RAA or the RSA is used. Most recent applications of the RPH method now compute $S_{0}$ as an $E_{i}$-dependent weighted average of $S_{0}$ computed with the RAA and computed with the $\mathrm{RSA},{ }^{162,178,179,527}$ as first done in ref. 527.

There are also uncertainties concerning the treatment of parallel translational motion. In the QD RPH method of Jackson and co-workers the $X$ and $Y$ DOFs are also treated with the SAHP approach. ${ }^{50}$ Values of $S_{0}$ for $(X, Y)$ differing from $\left(X^{\mathrm{TS}}, Y^{\mathrm{TS}}\right)$ are computed by estimating the energy difference $\Delta V(X, Y)$ between the barrier at $(X, Y)$ and $\left(X^{\mathrm{TS}}, Y^{\mathrm{TS}}\right)$ using a harmonic approximation, similar to eqn (17).$^{50}$ Comparison of RPH and AIMD results has shown that the method works well for $E_{i}$ close to $E_{\mathrm{b}}$, but not for high $E_{i \cdot}{ }^{89}$ As also noted in ref. 123, the problem is that the harmonic approximation tends to overestimate $\Delta V(X, Y)$ for $(X, Y)$ far away from the TS, which may result in underestimation of $S_{0}$ at high $E_{i}{ }^{89}$ The authors pointed out that the problem was not necessarily due to a sudden approximation being made for motion in $X$ and $Y$, but rather its implementation through the SAHP approximation.

Finally, for technical reasons the QD RPH method usually includes only a few excited states in the scattering basis set. While differences between results obtained with a basis set including up to two-quanta states and up to one-quantum states appear small on a log scale, they may in fact be substantial in specific cases (see e.g. Fig. 2 and 4 of ref. 89) and it is not completely clear how the results would change if the basis set were to be expanded beyond 2-quanta states. Also, it is obviously important to make a correct choice of the TS and associated minimum energy path, and there is an indication that this might have gone wrong in one specific case (compare the TS location and energy obtained for $\mathrm{H}_{2} \mathrm{O}+\mathrm{Ni}(100)$ in ref. 72 and ref. 98).

The great advantage of the RPH method is that it is the only QD method currently capable of including all vibrational states of $\mathrm{CH}_{4}$ in modeling its DC on metals. ${ }^{50,81}$ It predicts trends concerning the influence of the metal surface ${ }^{79,81}$ and of the initial vibrational state ${ }^{50,79}$ with quite high accuracy. The QD RPH method yields $S_{0}$ in good agreement with AIMD values for $\mathrm{CHD}_{3}+\operatorname{Pt}(111)^{51}$ (Fig. 29527). Using a PBE PES, the QD RPH method also yielded $S_{0}$ in generally good agreement with experiments $^{14,635}$ and $S_{0}$ computed for $\mathrm{CH}_{4}+\mathrm{Ni}(111)$ with a 12D PW91 PES using the 8D TDWP method $^{60}$ discussed in Section 4.4.3 (Fig. 30 30 ). However, note that the QD RPH method overestimated the experimental $S_{0}$ for $\mathrm{CH}_{4}$ in its vibrational ground state, that the PW91 PES based 8D TDWP method underestimated these experimental $S_{0}$, and that the RPH and 8D TDWP results for this state were quite different. Jackson and co-workers noted ${ }^{30}$ that the global PES used by Jiang and Guo and coworkers ${ }^{60}$ was constructed on the basis of a smaller surface unit cell resulting in a $E_{\mathrm{b}}$ too high by $0.1 \mathrm{eV}$. This explains part of the difference between the theoretical results for the vibrational ground state in Fig. 30, but not why the agreement obtained for the excited vibrational states is much better.

\subsection{RPMD}

In RPMD calculations ${ }^{636,637}$ classical nuclei are effectively treated as "ring polymers", and an isomorphism is exploited between 


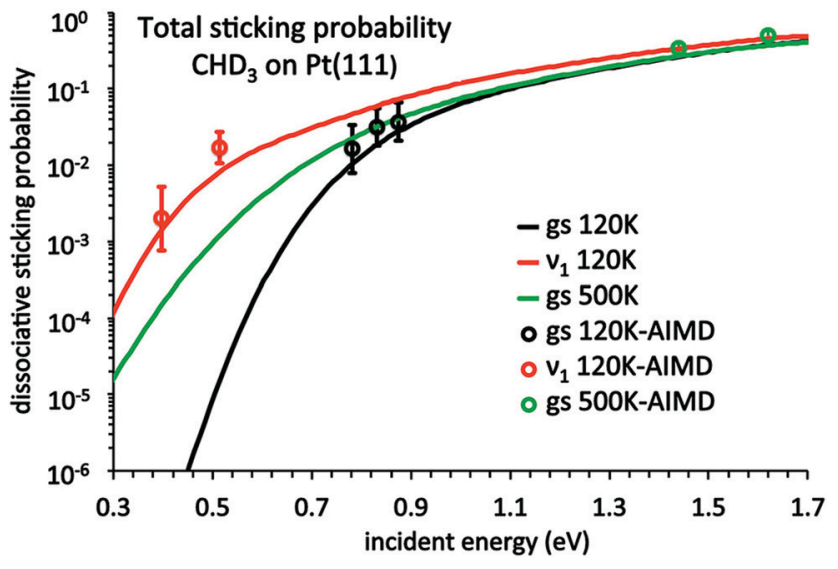

Fig. $29 \mathrm{~S}_{0}$ computed with the RPH method for $\mathrm{CHD}_{3}+\mathrm{Pt}(111)$ for the value of $T_{\mathrm{s}}$ and the initial vibrational state ( $\mathrm{gs}=$ ground state, and the $\nu_{1}=1$ vibrationally excited state) indicated are shown as a function of incident energy, and compared to values computed ${ }^{51}$ with DFMD ("AIMD", symbols with error bars). Reprinted from [H. Guo and B. Jackson, Mode-selective chemistry on metal surfaces: the dissociative chemisorption of $\mathrm{CH}_{4}$ on Pt(111), J. Chem. Phys., 2016, 144, 184709], with the permission of AIP Publishing.

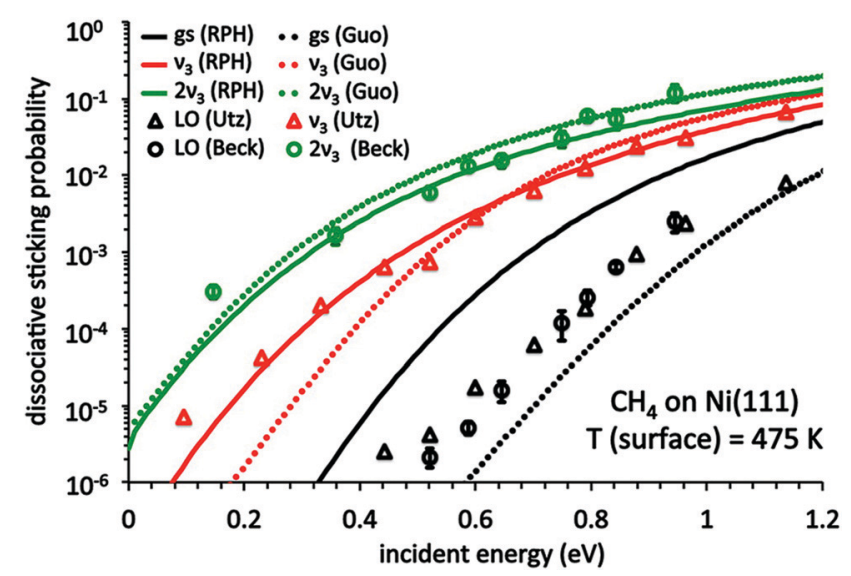

Fig. $30 S_{0}$ computed ${ }^{527}$ with the RPH method (solid lines) are compared with $S_{0}$ computed ${ }^{60}$ with $8 D$ QD (dotted lines) for $\mathrm{CH}_{4}$ dissociating on $\mathrm{Ni}(111)$ at $T_{\mathrm{s}}=475 \mathrm{~K}$. Results are shown for three initial vibrational states ("gs" is the ground state, " $\nu_{3}$ " is the $\nu_{3}=1$ state, and " $2 \nu_{3}$ " is the $\nu_{3}=2$ state). The theoretical results are compared to experimental results, ${ }^{14,635}$ where "LO" refers to "laser-off" conditions (vibrational states occupied according to $\left.T_{n}\right)$. Reprinted with permission from (H. Guo, A. Farjamnia and B. Jackson, Effects of lattice motion on dissociative chemisorption: toward a rigorous comparison of theory with molecular beam experiments, J. Phys. Chem. Lett., 2016, 7, 4576-4584). Copyright (2016) American Chemical Society.

these "P-bead" ring polymers and path integrals. ${ }^{638}$ In principle, RPMD time correlation functions and quantities derivable from these are exact in the limits of parabolic barriers, short time propagation, high temperature, and harmonicity. ${ }^{636}$ While the method has been designed for treating kinetics (i.e., for computing thermal rates), a variant called non-equilibrium RPMD (NE-RPMD) has been developed ${ }^{639}$ that can in principle be applied to computing $S_{0} \cdot{ }^{597}$
To our knowledge, NE-RPMD has only been applied in two published papers considering scattering from surfaces, i.e., a paper addressing scattering of $\mathrm{H}$-atoms from graphene, ${ }^{640}$ and a paper ${ }^{597}$ addressing $\mathrm{DC}$ in $\mathrm{H}_{2}+\mathrm{Cu}(111)$ and $\mathrm{D}_{2} \mathrm{O}+\mathrm{Ni}(111)$ at $300 \mathrm{~K}$. Only in the latter publication an effort was made to test the method against QD calculations, and to compare its performance to that of QCT calculations. ${ }^{597}$

The outcome of the tests is to some extent promising, but it is also inconclusive (see also Section 4.2). For example, at the investigated temperature of $\mathrm{H}_{2}(300 \mathrm{~K})$ the NE-RPMD $S_{0}$ agree better with the QD results for $\mathrm{H}_{2}+\mathrm{Cu}(111)$ than the QCT results for $E_{i}$ in which sticking occurs probably through tunneling $(\leq 0.5 \mathrm{eV})$ (see Fig. 15, noting that the NE-RPMD method cannot be used to obtain $S_{0}$ for the vibrational ground state, i.e., for $0 \mathrm{~K}$ ). However, for high $E_{i}$ the QCT values of $S_{0}$ are better. The authors note ${ }^{597}$ that problems also occurred with the NE-RPMD results at high $E_{i}$ for $\mathrm{H}$ on graphene, ${ }^{640}$ and attributed the problem to some replicas of $\mathrm{H}_{2}$ getting too close to the surface. ${ }^{597}$ Whatever the cause of the problem is, it is disturbing that NE-RPMD fails in a regime where the QCT performance is excellent, as NE-RPMD is used with the aim of getting better results than obtainable with QCT.

For $\mathrm{D}_{2} \mathrm{O}+\mathrm{Ni}(111)$, at the investigated temperature of $\mathrm{D}_{2} \mathrm{O}$ $(300 \mathrm{~K})$ the NE-RPMD $S_{0}$ agree much better with the two sets of "QD $300 \mathrm{~K}$ " results than the QCT ones, at all $E_{i}$ investigated (see Fig. 20). However, there are quite a few problems surrounding the comparison: (i) the QD result is not an exact benchmark, 7D QD results were used with the SAED approach to obtain approximate 9D results, and it is not clear how accurate this approximation is on the log scale of Fig. 20. (ii) In one set of QD $300 \mathrm{~K}$ results, the approximation had to be made that the vibrational efficacy $\eta$ of the higher vibrationally excited states should equal 1.0 (in fairness the results are not very sensitive to this value of $\eta) .{ }^{597}$ (iii) In the set of QD $300 \mathrm{~K}$ results, the QD $300 \mathrm{~K}$ results were obtained by multiplying the QD vibrational ground state results with the ratio of the QCT $300 \mathrm{~K}$ results divided by the QCT GS results. The latter approximation is highly suspect if, as suggested by the authors, the QCT GS results deviate from the QD results due to zpe violation. ${ }^{597}$

We finally note that the comparison made by the authors is not yet relevant to actual MB sticking experiments. For instance, $T_{\mathrm{N}}=2100 \mathrm{~K}$ is required to achieve an average $E_{i}$ of $0.5 \mathrm{eV}$ for $\mathrm{H}_{2}+$ $\mathrm{Cu}(111)$ (Fig. 15), and Hundt et al. used $T_{\mathrm{N}}=573 \mathrm{~K}$ to achieve an $E_{i}$ of $0.6 \mathrm{eV}$ for $\mathrm{D}_{2} \mathrm{O}+\mathrm{Ni}(111)$ (Fig. 20). Nevertheless, the results of Jiang and co-workers are quite interesting, in that they suggest that NE-RPMD might be used to address sticking for conditions under which QCT might fail due to problems with tunneling or zpe violation, for systems for which high-dimensional QD calculations are not yet within reach. As noted by the authors, ${ }^{597}$ NE-RPMD calculations might describe tunneling well, and not suffer from zpe violation. However, more studies are needed to establish the reliability of NE-RPMD for describing DC. Also, it is necessary to address the problem of how simulations can be performed of sticking under conditions where $T_{\text {vib }}$ and $T_{\text {rot }}$ differ widely, as would be expected to be the case in supersonic MB experiments on sticking of polyatomic molecules. ${ }^{597}$ 


\subsection{Computation of observables}

By far the most important observable to compute on the basis of SRP-DFT is the zero-coverage or initial sticking probability $S_{0}\left(E_{i}^{\mathrm{av}} ; T_{\mathrm{N}}\right)$. From now on we will assume that $E_{i}^{\mathrm{av}}$ is the $E_{i}$ averaged over the flux weighted velocity distribution of the MB obtained by expanding through a nozzle of temperature $T_{\mathrm{N}}$, but see below for further discussion. The calculation of $S_{0}\left(E_{i}^{\mathrm{av}} ; T_{\mathrm{N}}\right)$ starts with obtaining the Boltzmann averaged reaction probability $R\left(E_{i} ; T_{\mathrm{N}}\right)$ for a specific $E_{i}$ from the initial state selected reaction probabilities $R_{\mathrm{v}}\left(E_{i}\right)$. This is achieved by computing

$$
R\left(E_{i} ; T_{\mathrm{N}}\right)=\sum_{\boldsymbol{v}, \boldsymbol{j}} F_{\mathrm{B}}\left(\boldsymbol{v}, \boldsymbol{j}, T_{\mathrm{N}}\right) R_{v j}\left(E_{i}\right)
$$

Here, $\boldsymbol{v}$ is the vector of initial vibrational quantum numbers defining the initial vibrational state of the molecule, and $j$ is the vector of rotational quantum numbers defining the initial rotational state of the molecule. For example, $j$ would be $(J, K, M)$ for a prolate or symmetric top molecule, where $K$ is the projection of $J$ on the unique rotational axis. The Boltzmann factor $F_{\mathrm{B}}\left(v, j, T_{\mathrm{n}}\right)$ should take into account (i) effects of nuclear spin symmetry (e.g. even $j$ (para) states of $\mathrm{H}_{2}$ should be weighted with a factor 1 , and odd $j$ (ortho) states with a factor 3 ), and (ii) the fact that $T_{\text {vib }}$ and $T_{\text {rot }}$ may differ from $T_{\mathrm{N}}$, and from each other. For instance, for an $\mathrm{H}_{2} \mathrm{MB}$, $T_{\text {vib }} \approx T_{\mathrm{N}}$, and $T_{\text {rot }} \approx 0.8 T_{\mathrm{N}} \cdot{ }^{641-643}$ For $\mathrm{CH}_{4}$ beams, $T_{\text {rot }}$ may be much lower, e.g. $T_{\text {rot }} \approx 0.025 T_{\mathrm{N}}{ }^{644}$

To obtain $S_{0}\left(E_{i}^{\mathrm{av}} ; T_{\mathrm{N}}\right), R\left(E_{i} ; T_{\mathrm{N}}\right)$ needs to be averaged over the flux weighted velocity distribution of the MB according to

$$
S_{0}\left(E_{i}^{\mathrm{av}} ; T_{\mathrm{N}}\right)=\int_{v=0}^{v=\infty} f\left(v ; T_{\mathrm{N}}\right) R\left(E_{i} ; T_{\mathrm{N}}\right) \mathrm{d} v / \int_{v=0}^{v=\infty} f\left(v ; T_{\mathrm{N}}\right) \mathrm{d} v .
$$

In eqn (19), $E_{i}=\frac{1}{2} M v^{2}$. The flux weighted velocity distribution is best taken as ${ }^{645,646}$

$$
f\left(v ; T_{\mathrm{N}}\right) \mathrm{d} v=C v^{3} \exp \left[-\left(v-v_{0}\right)^{2} / \alpha^{2}\right] \mathrm{d} v .
$$

Here, $C$ is a constant, $v_{0}$ is the stream velocity, and $\alpha$ is the width of the velocity distribution. One can also employ a similar expression for the (skewed) distribution of $E_{i}$. Alternatively, some researchers have fitted their $E_{i}$ distributions to a Gaussian function according to ${ }^{646,647}$

$$
g\left(E_{i}\right)=\sqrt{2 \pi \sigma} \exp \left(-\left(E_{i}-E_{i}^{\mathrm{av}}\right)^{2} / 2 \sigma\right)
$$

where $\sigma$ is taken to depend on $E_{i}^{\text {av }}{ }^{647}$

To determine the $v_{0}$ and $\alpha$ parameters, the best practice is to fit TOF distributions of the molecules in the beam according to

$$
G(t)=C \frac{L^{3}}{t^{4}} \exp \left[-\left(\frac{\frac{L}{t}-v_{0}}{\alpha}\right)^{2}\right]
$$

while taking into account the ion-flight time in the quadrupole mass spectrometer assumed to be used when writing eqn (22), the chopper function, etc. ${ }^{646}$ In eqn (22), $L$ is the length of the flight path. The $E_{i}$ averaged over the flux weighted velocity distribution (eqn (20)) may be obtained analytically from ${ }^{648}$

$$
E_{i}^{\mathrm{av}}=\frac{1}{2} M\left\langle v^{2}\right\rangle=\frac{1}{2} M \frac{\frac{15}{4} \alpha^{4}+5 \alpha^{2} v_{0}^{2}+v_{0}^{4}}{\frac{3}{2} \alpha^{2}+v_{0}^{2}} .
$$

Many experimentalists present their measured $S_{0}$ as a function of $E_{i}^{\text {av }}$, a practice that has been followed in for instance much of the work on $\mathrm{H}_{2}+\mathrm{Cu}(111) .{ }^{12,43,641,649}$ However, not all experimentalists follow this practice. Other researchers use an approximate method for extracting the average $E_{i}$ directly from the peak in the measured TOF spectrum $G(t)$ (eqn (22)). In this method, the peak condition is used to determine the time at which the peak occurs in the TOF spectrum, for flight length $L$, which we will call $t_{\mathrm{M}}^{\mathrm{L}}$. If there are no time delays associated with the method to detect $\mathrm{H}_{2}$, the velocity that may be associated with the $\mathrm{MB}$ can be written

$$
v_{\mathrm{M}}=\frac{L}{t_{\mathrm{M}}^{\mathrm{L}}} .
$$

This velocity may be written in terms of the parameters $v_{0}$ and $\alpha$ as $^{648}$

$$
v_{\mathrm{M}}=\frac{v_{0}}{2}+\sqrt{\frac{v_{0}^{2}}{4}+2 \alpha^{2}} .
$$

These experimentalists then obtain the $E_{i}$ of the molecule, as a function of which they present their $S_{0}\left(E_{i} ; T_{\mathrm{N}}\right)$, according to $E_{i}^{\mathrm{M}}=1 / 2 M v_{\mathrm{M}}{ }^{2}$. Comparison of eqn (25) to eqn (23) shows that $E_{i}^{\mathrm{M}} \neq E_{i}^{\mathrm{av}}$, and we have seen that $E_{i}^{\mathrm{M}}$ may overestimate $E_{i}^{\mathrm{av}}$ by as much as $3 \%$ for $\mathrm{H}_{2}$ beams. Still worse errors may be obtained if $v_{M}$ is taken as an estimate of $v_{0}$, as eqn (24) shows. Unfortunately, not all experimentalists state to which expression their $E_{i}$ used in plots of $S_{0}$ correspond, and it is even rarer that experimentalists report the $v_{0}, \alpha$, and $T_{\mathrm{N}}$ parameters corresponding to their experiments. This often makes it necessary to guess these parameters when simulating these experiments. ${ }^{163}$

It is hard to overstate the importance of knowing the MB parameters when simulating experiments, especially when dealing with activated DC of $\mathrm{H}_{2}$. Fig. 31 shows two sets of measured $S_{0}$ for $\mathrm{H}_{2}+\mathrm{Cu}(111)$ that show dramatic differences. The $S_{0}\left(E_{i}^{\text {av }} ; T_{\mathrm{N}}\right)$ measured by Berger et al. ${ }^{649}$ were an order of magnitude larger at similar $E_{i}^{\text {av }}$ than the $S_{0}\left(E_{i}^{\text {av }} ; T_{\mathrm{n}}\right)$ measured by Rettner et al. ${ }^{641}$ This was because the $E_{i}$ distributions with which the $R\left(E_{i} ; T_{\mathrm{n}}\right)$ (the solid black curve in Fig. 32) needs to be multiplied, with an expression analogous to eqn (19) but involving incidence energies rather than incident velocities, were much broader in the experiments of Berger et al. ${ }^{649}$ (solid line with circles in Fig. 32) than in the experiments of Rettner et al. ${ }^{641}$ (dashed curves in Fig. 32) at similar $T_{\mathrm{n}}$. Fig. 31 and 32 serve as powerful demonstrations of why experimentalists using MBs to determine sticking should document the parameters characterizing their beams.

One can also compute state-to state scattering probabilities for scattering back to the gas phase from some initial state to some specific final state of the molecule. The most fully state 


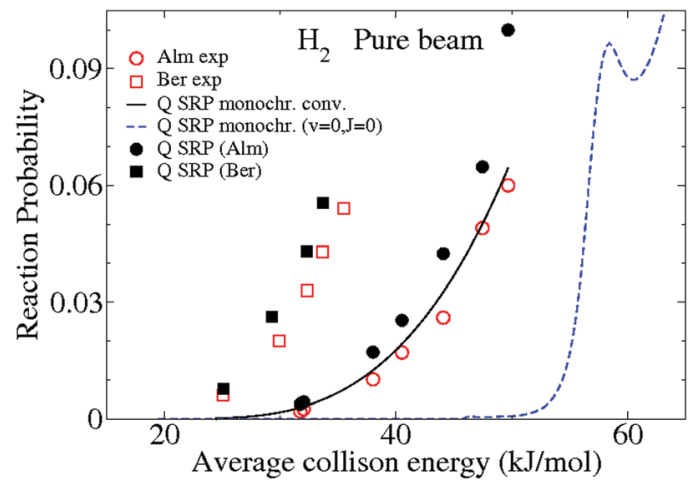

Fig. $31 \mathrm{~S}_{0}$ computed ${ }^{159}$ with the SRP-DF for $\mathrm{H}_{2}+\mathrm{Cu}$ (111) (black squares and circles in the simulations of the molecular beam sticking experiments by Berger et al. and by Auerbach and co-workers, respectively) are compared with the $S_{0}$ measured for the same system by Berger et al. ${ }^{649}$ (red squares) and by Auerbach and co-workers ${ }^{641}$ (red circles). The dashed blue line shows the $R_{v=0, j=0}\left(E_{j}\right)$ and the solid black line shows the $R\left(E_{i} ; T_{n}\right)$ computed with SRP-DFT. Taken from ref. 159

resolved scattering probability that can be computed is the probability for vibrationally, rotationally and diffractionally inelastic scattering $P_{v j \rightarrow v^{\prime} j^{\prime} n m}\left(E_{i}\right)$. Here, $n$ and $m$ are the quantum numbers for diffraction. In a TDWP calculation, these probabilities are computed from $S$-matrix elements, which may be calculated in a scattering amplitude formalism. ${ }^{650,651}$ Probabilities for rovibrationally inelastic scattering can be obtained by summing over $n$ and $m$ :

$$
P_{v j \rightarrow v^{\prime} j^{\prime}}\left(E_{i}\right)=\sum_{n, m} P_{v j \rightarrow v^{\prime} j^{\prime} n m}\left(E_{i}\right) .
$$

One way the $R_{v j}\left(E_{i}\right)$ can be obtained is by summing rovibrationally inelastic scattering probabilities and subtracting from 1 , i.e.,

$$
R_{v j}\left(E_{i}\right)=1-\sum_{v^{\prime} j^{\prime}} P_{v j \rightarrow v^{\prime} j^{\prime}}\left(E_{i}\right) .
$$

However, the $R_{v j}\left(E_{i}\right)$ may also be computed by starting a TDWP calculation for an incoming initial $(v j)$ state and analyzing the reactive flux for through a surface taken at a large enough, fixed value of the dissociative co-ordinate $r .{ }^{652}$ For comparison to experiments one is usually not interested in fully resolving the probabilities with respect to the $M$ and $M^{\prime}$ quantum numbers, and an expression for the state-to-state scattering probabilities involving the reduced rotational quantum number vector $\boldsymbol{j}_{\mathrm{r}}$ ( $\boldsymbol{j}$ with $M$ taken out) may be obtained by degeneracy averaging

$$
P_{v j_{\mathrm{r}} \rightarrow v^{\prime} j_{\mathrm{r}}^{\prime}}\left(E_{i}\right)=\sum_{M, M^{\prime}} P_{v j_{\mathrm{r}} M \rightarrow v^{\prime} j_{\mathrm{r}}^{\prime} M^{\prime}}\left(E_{i}\right) /(2 J+1) .
$$

Similarly, the $R_{v j}\left(E_{i}\right)$ (eqn (27)) are usually not resolved with respect to $M$, and one may also use eqn (27) with $\boldsymbol{j}$ replaced by $\boldsymbol{j}_{\mathrm{r}}$ for both the initial and final state. Probabilities for vibrationally inelastic scattering can be obtained from

$$
P_{v j_{\mathrm{r}} \rightarrow v^{\prime}}\left(E_{i}\right)=\sum_{j_{\mathrm{r}}^{\prime}} P_{v j_{\mathrm{r}} \rightarrow v^{\prime} j_{\mathrm{r}}^{\prime}}\left(E_{i}\right)
$$

The reaction probability may also depend on the initial rotational polarization, i.e., on the initial distribution of $M^{17,18}$
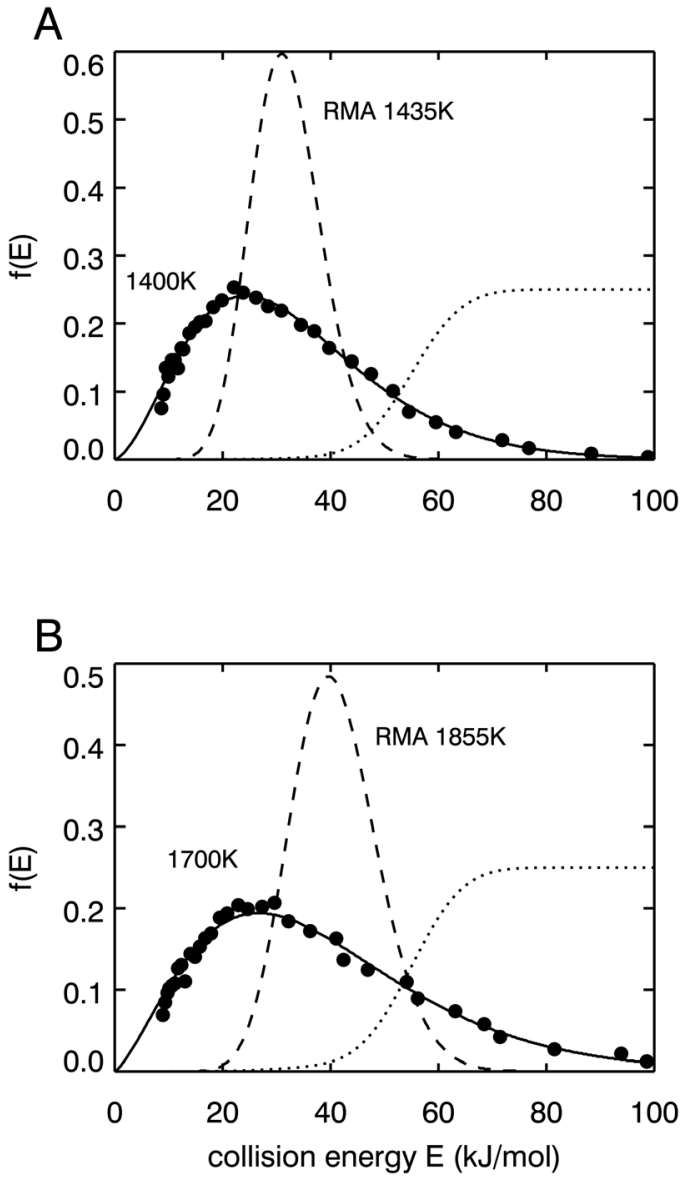

Fig. 32 Normalised incidence energy distributions characterising pure $\mathrm{H}_{2}$ beams, as used by Berger et al. ${ }^{649}$ (solid lines) and by Auerbach and coworkers ${ }^{641}$ (labelled RMA, dashed lines) are shown for similar $T_{n}$, i.e., $T_{n} \approx$ $1400 \mathrm{~K}(\mathrm{~A})$ and $T_{\mathrm{n}} \approx 1700 \mathrm{~K}(\mathrm{~B})$. Also shown is the function $R\left(E_{i} ; T_{\mathrm{n}}\right)$ as determined by the Almaden group ${ }^{641}$ neglecting the effect of velocity averaging (dotted lines). Reprinted from the Supporting Online Material for ref. 43.

For instance, from the $R_{v j}\left(E_{i}\right)$ one may compute the rotational quadrupole alignment parameter $A_{0}^{(2)}(J)$ of the reacting molecules according to

$$
\begin{aligned}
& A_{0}^{(2)}(J) \\
& \quad=\sum_{M}\left(R_{v j_{\mathrm{r}} M}\left(E_{i}\right)\left[3 M^{2}-J(J+1)\right] /[J(J+1)]\right) / \sum_{M} R_{v j_{\mathrm{r}} M}\left(E_{i}\right)
\end{aligned}
$$

Measured $A_{0}^{(2)}(J)$ contain information on how the reaction depends on the alignment of the molecule with respect to the surface, which may be parallel $(M=J)$ or "end-on" $(M=0)$.

Usually the $R_{v j}\left(E_{i}\right)$ are not directly measured in MB experiments, but instead extracted from associative desorption experiments. ${ }^{12,46}$ These measure translational energy $\left(E_{\mathrm{t}}\right)$ distributions of desorbing molecules $P_{\text {des }}\left(\boldsymbol{v}, \boldsymbol{j} ; E_{\mathrm{t}}\right)$. Assuming detailed balance, with $E_{\mathrm{t}}=E_{i}$ these may be related to the $R_{v j}\left(E_{i}\right)$ according to

$$
P_{\mathrm{des}}\left(\boldsymbol{v}, \boldsymbol{j} ; E_{i}\right) \propto E_{i} \exp \left[-E_{i} / k T_{\mathrm{s}}\right] R_{v j}\left(E_{i}\right)
$$


The $A_{0}^{(2)}(J)$ are usually also determined in associative desorption experiments. ${ }^{17}$ Note that the $R_{\boldsymbol{v} j}\left(E_{i}\right)$ that may be obtained by inverting eqn (31) on the basis of measured desorption fluxes are in general not yet normalized, although they may be normalized relative to one another, ${ }^{23}$ or on an absolute scale by either expressing measured $S_{0}$ in terms of the $R_{v j}\left(E_{i}\right)$ obtained by inverting eqn $(31)^{12,641}$ or by equating measured $R_{v j}\left(E_{i}\right)$ to computed values at a specific value of $E_{i}{ }^{194}$ Also note that it is possible to compute the (unnormalised) energyand rovibrational state-resolved associative desorption flux $P_{\text {des }}\left(\boldsymbol{v}, j ; E_{i}\right)$ directly, by running trajectories from the transition state, ${ }^{653-656}$ which was recently done for $\mathrm{H}_{2}+\mathrm{Cu}(111)$ using AIMD and AIMDEF. ${ }^{657}$

The $R_{v j}\left(E_{i}\right)$ are often ${ }^{12,641}$ fitted with the error function expression

$$
R_{v j}\left(E_{i}\right)=\frac{A_{v j}}{2}\left[1+\operatorname{erf}\left(\frac{E_{i}-E_{0}^{v j}}{W_{v j}}\right)\right]
$$

In eqn (32), $E_{0}$ is usually called the effective barrier height, i.e., the $E_{i}$ at which the $R_{v j}\left(E_{i}\right)$ equals half its saturation value $A$, and $W$ is a width parameter. A similar expression using the tanh function instead of the error function has also been used. It is also possible to use an asymmetric form by employing the generalized logistic (LGS) function ${ }^{170}$

$$
R_{v j}\left(E_{i}\right)=A /\left[1+\nu \exp \left(-\frac{E-E_{0}^{v j^{\prime}}}{W^{\prime}}\right)\right]^{\frac{1}{\nu}}
$$

In experiments it may be difficult to determine $A$ accurately (see our remarks above), and methods of comparing theory to experiment when computing the "experimental" parameters characterizing $R_{\mathrm{v} j}\left(E_{i}\right)$ may differ depending on whether or not theoretical information is used to characterize these experimental parameters. ${ }^{658}$

Finally, in associative experiments it is also possible to determine associative desorption energies (ADEs) according to ${ }^{12,46,641}$

$$
\left\langle E_{\mathrm{t}}(\boldsymbol{v}, \boldsymbol{j})\right\rangle=\frac{\int E_{i}^{2} \exp \left[-E_{i} / k T_{\mathrm{s}}\right] R_{\boldsymbol{v} j}\left(E_{i}\right) \mathrm{d} E_{i}}{\int E_{i} \exp \left[-E_{i} / k T_{\mathrm{s}}\right] R_{\boldsymbol{v} j}\left(E_{i}\right) \mathrm{d} E_{i}} .
$$

The ADEs may depend rather strongly on $T_{\mathrm{s}}$, but their determination does not require the normalization of the $R_{v j}\left(E_{i}\right)$. The observables discussed here do not represent a complete list, but the most important quantities have been dealt with, and expressions of other observables may be found in specialized literature.

\section{Results: systems for which SRP-DFs exist, and use of SRP-DFs}

In this section, we will first discuss results for the seven systems for which SRP-DFs and accurate $E_{\mathrm{b}}$ have been derived $\left(\mathrm{H}_{2}+\right.$ $\mathrm{Cu}(111), \mathrm{Cu}(100), \mathrm{Cu}(110)$, and $\mathrm{Pt}(111)$, and $\mathrm{CHD}_{3}+\mathrm{Ni}(111)$, $\mathrm{Pt}(111)$, and $\mathrm{Pt}(211)$ ) in Section 5.1. We consider systems for which c-SRP DFs have been derived $\left(\mathrm{H}_{2}+\mathrm{Pt}(211), \mathrm{Ru}(0001)\right.$, $\mathrm{Ni}(111)$, and $\mathrm{N}_{2}+\mathrm{Ru}(0001)$ ) in Section 5.2. Systems for which attempts (whether advertised as such or not) have so far not been successful at delivering (candidate) SRP-DFs are discussed in Section 5.3. In Section 5.4 we discuss calculations on specific systems that have used SRP-DFs developed for related systems.

\subsection{Systems for which SRP-DFs have been derived}

5.1.1. $\mathbf{H}_{2}+\mathbf{C u}(\mathbf{1 1 1})$. The first system for which a SRP-DF was derived was $\mathrm{H}_{2}+\mathrm{Cu}(111) .{ }^{43}$ The SRP-DF was fitted ${ }^{43}$ by demanding QCT calculations to reproduce $S_{0}$ measured $^{12}$ for $\mathrm{D}_{2}+\mathrm{Cu}(111)$ obtained with $\mathrm{MB}$ experiments using seeding in $\mathrm{H}_{2}$ to achieve high $E_{i}$ (Fig. 6). The original SRP-DF fitted existed of a weighted average of the PW91 ${ }^{312}(57 \%)$ and RPBE $^{196}(43 \%$, $\alpha=0.43)$ GGA DFs. With the fitted SRP-DF, which we call the SRP43 DF here, the $S_{0}$ measured for $\mathrm{H}_{2}+\mathrm{Cu}(111)$ by two different groups ${ }^{641,649}$ could be reproduced in spite of the large discrepancies between the two experimental datasets (Fig. 31), and these discrepancies could be attributed to differences between the velocity distributions of the MBs used (Fig. 32, see also Section 4.6). ${ }^{43}$ QD calculations using the SRP43 PES could also reproduce the measured ${ }^{659}$ ratio of rotationally inelastic and elastic scattering probabilities $P(v=1, j=0 \rightarrow v=$ $1, j=2) / P(v=1, j=0 \rightarrow v=1, j=0)$ to within chemical accuracy (see Fig. 3 of ref. 43 ).

The $E_{0}^{v j}$ (see eqn (32)) measured ${ }^{641}$ for $\mathrm{H}_{2}$ associatively desorbing from $\mathrm{Cu}(111)$ could also be reproduced with chemical accuracy (with a MUE of only $2.5 \mathrm{~kJ} \mathrm{~mol}^{-1}$, see Fig. 33A). ${ }^{43}$ A similar result $\left(\mathrm{MUE}=3.2 \mathrm{~kJ} \mathrm{~mol}^{-1}\right.$ ) held for $\mathrm{D}_{2}+$ $\mathrm{Cu}(111)$, although this required the use of an asymmetric fit function for the $R_{v j}\left(E_{i}\right) .{ }^{170}$ However, the calculations did not reproduce the experimentally observed trend that for a given $v$ the $E_{0}^{v j}$ first increase and then decrease with $j$ for both $\mathrm{H}_{2}$ (see Fig. 33A) and $\mathrm{D}_{2}$ (see Fig. 13 of ref. 170). A point of subtle interest is that the calculations with the SRP43 DF overestimate the reactivity obtained in the sticking experiments (Fig. 6) while they underestimate the reactivity obtained in the associative desorption experiments (Fig. 33A). The dynamics calculations with the SRP43 DF reproduce ADEs measured ${ }^{660}$ in associative desorption for $T_{\mathrm{S}}=370 \mathrm{~K}$ and the $(v=0, j=1,3$, and 5) states with chemical accuracy (Fig. 33B, MUE $=2 \mathrm{~kJ} \mathrm{~mol}^{-1}$ ). ${ }^{159}$ However, chemical accuracy was not achieved (MUE = $\left.13.5 \mathrm{~kJ} \mathrm{~mol}^{-1}\right)^{159}$ for the dynamics calculations (which were performed with the BOSS model) comparing to associative desorption experiments for $T_{\mathrm{S}}=925 \mathrm{~K}^{641}$ (Fig. 33B). The difference is due to the ADE decreasing with increasing $T_{\mathrm{s}}$ due to the broadening of the reaction probability curve with increasing $T_{\mathrm{s}},{ }^{170,175,641}$ and this could only partly be corrected for $\left(\mathrm{MUE}=6.0 \mathrm{~kJ} \mathrm{~mol}^{-1}\right)$ by attempting to extrapolate the measured ADEs to $T_{\mathrm{s}}=120 \mathrm{~K} .{ }^{159}$ In summary, the computed $E_{0}^{v j}$ and ADEs were too high compared to the experiments of Rettner and Auerbach and co-workers. ${ }^{12,641}$ However, comparison ${ }^{46}$ to experiments of Comsa and David ${ }^{661}$ suggests that the problem may also lie with the associative desorption experiments, ${ }^{12,641}$ which may have overestimated the ADEs by about $10 \%$.

Measured $^{17} A_{0}^{(2)}(J)$ (eqn (30)) could not be reproduced with dynamics calculations using the BOSS model ${ }^{159,175}$ (Fig. 34). However, introducing $T_{\mathrm{s}}$ and surface motion via DFMD calculations lead to a considerable improvement (Fig. 34): with DFMD the $A_{0}^{(2)}(J)$ measured for $(v=1, j=6)$ could be reproduced accurately, 


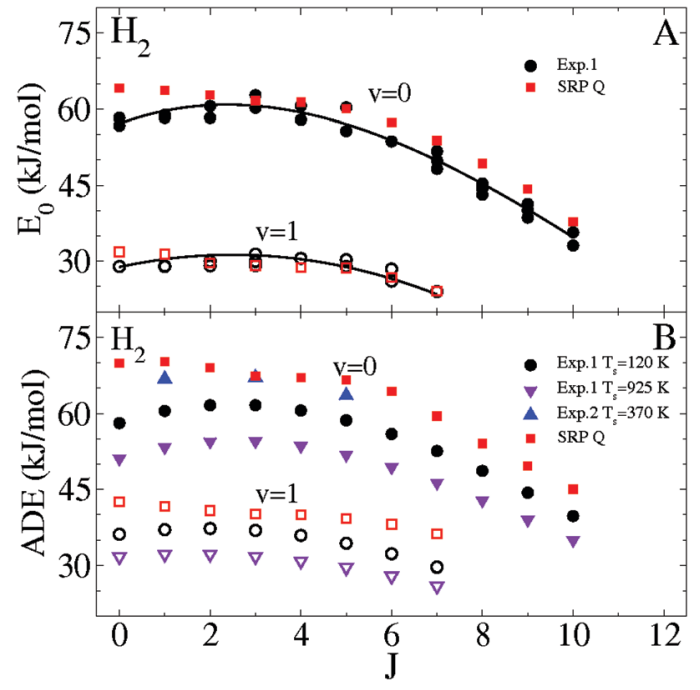

Fig. 33 Results for associative desorption of $\mathrm{H}_{2}$ from $\mathrm{Cu}(111)$. (A) Effective barrier heights $E_{0}(v, J)$ computed ${ }^{159}$ with QD using a SRP PES are compared with values extracted from experiments for repeated measurements. ${ }^{641}$ (B). ADEs computed ${ }^{159}$ with QD using a SRP PES are compared with values extracted from experiments. ${ }^{641,660}$ "Exp.1" refers to ref. 641 and "Exp.2" refers to ref. 660. Reproduced from ref. 159 with permission from the PCCP Owner Societies.

although the DFMD calculations still overestimated the experimental results for $(v=0, j=11) \cdot{ }^{175}$ To enable the DFMD calculations, a new SRP-DF (the SRP48 DF) had to be fitted that was however very similar to the old one, with the new one being a weighted average of the PBE $(52 \%)$ and the RPBE $(48 \%$, $\alpha=0.48)$ GGA DFs. ${ }^{175}$

Calculations within the BOSS model ${ }^{177}$ have also failed to reproduce experiments on vibrational excitation, i.e., the short time peak occurring in the measured ${ }^{581}$ TOF spectrum shown in Fig. 35, which is due to vibrational excitation of $(v=0, j)$ states to $(v=1, j=3)$. With the assumption that $30 \%$ of the incident kinetic energy was transferred to the surface $f(K)=0.3$ in Fig. 35) and considering the computed scaling with normal and total incidence energy, the computed vibrational excitation probabilities still had to be multiplied with a factor 2.6 to reproduce experiment. ${ }^{177}$ Subsequent DFMD and GLO + F calculations suggest that the energy loss to surface phonons (computed in the range 18-26\%) was somewhat smaller than the estimated $30 \%,{ }^{168}$ which does not help with explaining the discrepancy observed in Fig. 35. This later work also raised another problem. In our earlier analysis, ${ }^{177}$ we had noted that increasing $T_{\mathrm{s}}$ from 400 to $700 \mathrm{~K}$ in the experiment raises the height of the gain peak, suggesting an increase of the vibrational excitation probabilities by about $20 \%$. Extrapolating down to $0 \mathrm{~K}$ and equating a $0 \mathrm{~K}$ to a static surface, this led us to believe that we could multiply our computed vibrational excitation probabilities with a factor 1.2 to account for the use of the BOSS model (assumed to mimic a $0 \mathrm{~K}$ surface) when modeling an experiment with $T_{\mathrm{s}}=400 \mathrm{~K}$ (as in Fig. 35 ). ${ }^{177}$ However, simultaneously allowing the surface atoms to move and imposing $T_{\mathrm{s}}=400 \mathrm{~K}$ was instead found to diminish the computed vibrational excitation probabilities. ${ }^{168}$ In qualitative agreement with experiment the

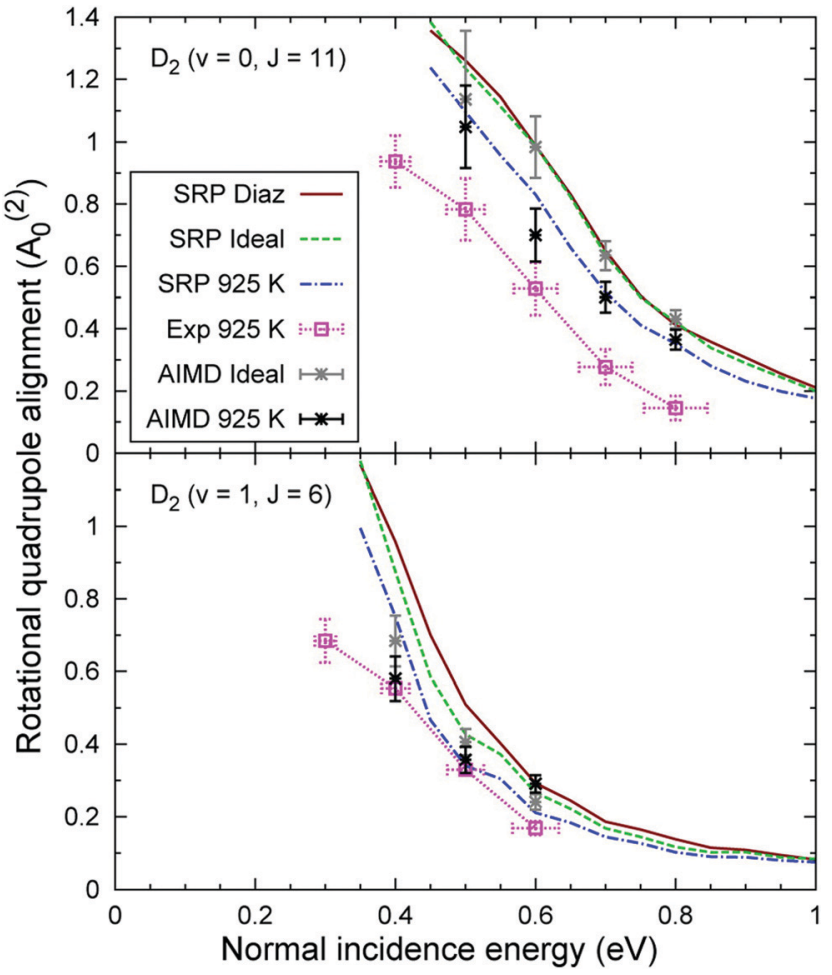

Fig. 34 Shown are the $A_{0}^{(2)}(\mathrm{J})$ as measured ${ }^{17}$ " $(\operatorname{Exp} 925 \mathrm{~K})$ " and computed for $D_{2}+C u(111)$ for two rovibrational states. The "SRP Diaz" and "SRP ideal" results were computed ${ }^{159,172}$ with QCT for an ideal Cu(111) surface with a lattice constant and lattice interlayer distances corresponding to $0 \mathrm{~K}$. The "SRP $925 \mathrm{~K}^{\text {" results were computed }}{ }^{172}$ with QCT for an ideal $\mathrm{Cu}(111)$ surface with a lattice constant and lattice interlayer distances corresponding to $925 \mathrm{~K}$. The "AIMD ideal" and the "AIMD $925 \mathrm{~K}$ " results were computed ${ }^{175}$ with DFMD for an ideal Cu(111) surface with a lattice constant and lattice interlayer distances corresponding to $0 \mathrm{~K}$ and for a mobile $\mathrm{Cu}(111)$ surface equilibrated at $925 \mathrm{~K}$, respectively. All theoretical results were obtained with the SRP48 DF ${ }^{175}$ except the "SRP Diaz" results, which were obtained using the original SRP functional. ${ }^{43}$ Reprinted with permission from (A. Mondal, M. Wijzenbroek, M. Bonfanti, C. Díaz and G. J. Kroes, Thermal lattice expansion effect on reactive scattering of $\mathrm{H}_{2}$ from Cu(111) at $T_{\mathrm{s}}=925 \mathrm{~K}$, J. Phys. Chem. A, 2013, 117, 8770-8781). Copyright (2013) American Chemical Society.

short time peak in Fig. 35 does increase with $T_{\mathrm{S}}$ if ehp excitation is modeled, showing that the measured increase of vibrational excitation with $T_{\mathrm{S}}$ was due to the effect of ehps instead of phonons. ${ }^{168}$ However, the lack of quantitative agreement with the experiments on vibrational excitation remains the biggest puzzle presently left for $\mathrm{H}_{2}+\mathrm{Cu}(111)$.

For $\mathrm{H}_{2}+\mathrm{Cu}(111)$ additional SRP-DFs have been derived. Wijzenbroek et al. found ${ }^{150}$ that the optPBE-vdW1 DF, which combines the optPBE GGA exchange $\mathrm{DF}^{331}$ with the vdW1 correlation $\mathrm{DF},{ }^{326}$ describes the experiments on reaction of $\mathrm{D}_{2}$ on $\mathrm{Cu}(111)^{12}$ shown in Fig. 6 even better than the SRP48 DF. ${ }^{175}$ Subsequent calculations by Jiang and co-workers ${ }^{467}$ also showed a chemically accurate description of experiments on $\mathrm{H}_{2}+$ $\mathrm{Cu}(111),{ }^{649}$ making this functional an SRP-DF (see also below). Additionally, three mGGAs of the "made simple" type have been constructed that all give a chemically accurate description of $S_{0}$ of $\mathrm{H}_{2}$ on $\mathrm{Cu}(111){ }^{152}$ These mGGAs all outperformed other 


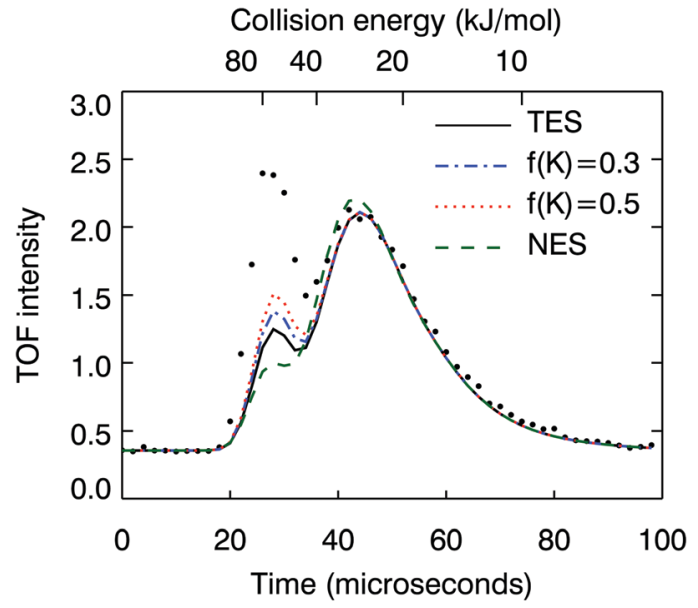

Fig. 35 The measured TOF spectrum for scattering of $\mathrm{H}_{2}$ into its $(v=1$, $J=3$ ) state $^{581}$ (black dots) is compared to TOF spectra obtained ${ }^{177}$ from normal incidence QD calculations assuming normal energy scaling (NES), total energy scaling (TES), assuming TES and 30\% energy loss to the surface relative to the translational energy available in scattering from a static surface $(f(K)=0.3)$, and assuming TES and $50 \%$ energy loss to the surface $(f(K)=0.5)$. Figure taken from ref. 177 .

well-known mGGAs on sticking of $\mathrm{D}_{2}$ on $\mathrm{Cu}(111)$, such as the TPSS, ${ }^{662}$ revTPSS, ${ }^{199}$ and SCAN ${ }^{339}$ DFs. The performance of the latter three mGGAs and of the GGA PBE and RPBE DFs on sticking of $\mathrm{D}_{2}+\mathrm{Cu}(111)$ is compared in Fig. 36. Interestingly, the maximally constrained SCAN DF showed the worst agreement with experiment; the SCAN DF also gives a mediocre description of other molecule-metal surface systems ${ }^{152}$ (see also Table 2). An advantage of the made simple DFs tested in ref. 152 is that they also provided a very good description of late TMs as demonstrated for bulk $\mathrm{Cu}, \mathrm{Ag}$, $\mathrm{Au}$, and $\mathrm{Pt}$ and the $\mathrm{Cu}(111)$ and $\operatorname{Ag}(111)$ surface, with the bulk metal description being of similar quality as obtained with the PBEsol DF developed specifically for solid state applications. ${ }^{314}$

Very recently SRP-DFs have been designed ${ }^{658}$ that combine GGA exchange with vdW2 correlation. $^{327}$ The exchange

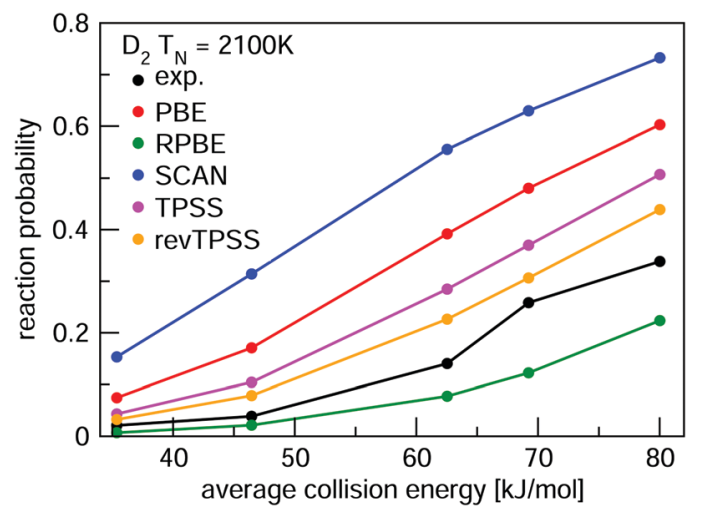

Fig. 36 Values of $S_{0}$ measured ${ }^{12}$ in molecular beam experiments for $D_{2}+$ $\mathrm{Cu}(111)$ are compared to computed ${ }^{152}$ values using PESs based on DFT calculations using the PBE, ${ }^{195}$ the RPBE, ${ }^{196}$ the SCAN, ${ }^{339}$ the TPSS, ${ }^{662}$ and the rev-TPSS ${ }^{199}$ DFs. Taken from ref. 152 (https://pubs.acs.org/doi/10.1021/acs. jpca.9b02914). Further permission requests to be directed to the ACS. functionals found to perform well combined $68 \%$ of $\mathrm{B} 86 \mathrm{r}^{663}$ exchange with 32\% RPBE $^{196}$ exchange (B86SRP68-DF2), 63\% PBEsol $^{314}$ exchange with 37\% RPBE ${ }^{196}$ exchange (SRPsol63-DF2), while also the SRP-DF for $\mathrm{H}_{2}+\mathrm{Pt}(111)^{156}$ (PBE $\alpha 57$, see below) was tested. All three vdW2 DFs constructed described sticking experiments on $\mathrm{H}_{2}$ and $\mathrm{D}_{2}+\mathrm{Cu}(111)$ and associative desorption experiments on $\mathrm{H}_{2}+\mathrm{Cu}(111)$ with chemical accuracy. ${ }^{658}$

The SRP43 DF ${ }^{43}$ has been used in electronic structure calculations exploring how surface atom motion influences the interaction of $\mathrm{H}_{2}$ on $\mathrm{Cu}(111)$. Motions of second layer $\mathrm{Cu}$ atoms perpendicular to the surface and of first layer $\mathrm{Cu}$ atoms parallel to the surface exhibit electronic coupling to $\mathrm{H}_{2}$ (affecting the $E_{\mathrm{b}}$ for dissociation), while motion of first layer $\mathrm{Cu}$ atoms perpendicular to the surface was found to exhibit mechanical coupling to $\mathrm{H}_{2}$ (affecting the barrier location). ${ }^{664}$ Adding these four coordinates to a dynamical model may well suffice for a dynamical treatment of molecular and surface atom motion. ${ }^{664}$ Subsequent 7D and $6+1 \mathrm{D}$ calculations using the PSA to describe the effect of second-layer surface atom motion normal to the surface showed an excellent performance of the PSA. ${ }^{171}$ The effect of phonons on reactive scattering of $\mathrm{H}_{2}$ from $\mathrm{Cu}(111)$ has also been explored with the SCM model ${ }^{531}$ (see Section 4.1.2) and an improved version of this model. ${ }^{160}$ The earlier work ${ }^{531}$ used the SRP43 DF, ${ }^{43}$ while the later work ${ }^{160}$ employed the SRP48 DF. ${ }^{175}$ The SRP43 DF ${ }^{43}$ has also been used to assess the validity of a specific PES representation suitable to MCTDH calculations, ${ }^{176}$ and to assess the validity of the SAED approximation. ${ }^{102}$

The SRP48 $\mathrm{DF}^{175}$ has been used in studies ${ }^{172}$ of how thermal lattice expansion affects $S_{0}$ and $A_{0}^{(2)}(J)$, a question that had before received little attention. The calculations showed an important effect on the sticking, with thermal lattice expansion promoting reaction and reducing $A_{0}^{(2)}(J) .{ }^{172}$ The bulk and surface lattice expansion accounts ${ }^{172}$ for much of the effect on the $A_{0}^{(2)}(J)$ of allowing the surface atoms to move, as previously obtained from DFMD calculations ${ }^{175}$ (see Fig. 34). An important effect is that the distance between the top two layers increases with $T_{\mathrm{s}}$, as known from experiments. ${ }^{665}$ The calculations suggest that attempts to model the effect of $T_{\mathrm{S}}$ on reaction of $\mathrm{H}_{2}$ on $\mathrm{Cu}(111)$ may fail if surface expansion is not modeled. ${ }^{172}$

The SRP48 $\mathrm{DF}^{175}$ was also used ${ }^{169}$ to understand the observation $^{169}$ that rotational polarization has a larger effect on elastic scattering of cold $\mathrm{H}_{2}$ from a stepped $\mathrm{Cu}(115)$ than from a flat $\mathrm{Cu}(111)$ surface. The calculations showed that the corrugation of the $\mathrm{H}_{2}-\mathrm{Cu}$ interaction is much more dependent on the alignment of $\mathrm{H}_{2}$ with respect to the stepped than to the flat surface. ${ }^{169}$

The SRP $43^{43}$ and SRP $48{ }^{175}$ DFs were also used to investigate the effect of ehp excitation on rovibrationally inelastic scattering ${ }^{57,174}$ and on reactive scattering. ${ }^{57}$ The use of both the LDFA and the ODF models of EF was investigated in ref. 57. An important result $^{57}$ was that, regardless of whether the LDFA or ODF was used, additionally modeling the effect of ehp excitation improved the agreement with sticking experiments ${ }^{12,641}$ (Fig. 13). The calculations predicted that the best test of which model should best describe the effect of ehp excitation should be to measure vibrational deexcitation from specific $(v=2, j)$ states to specific $\left(v=1, j^{\prime}\right)$ states of $\mathrm{H}_{2}$ and $\mathrm{D}_{2}{ }^{57}$ 
Additionally, the optPBE-vdW ${ }^{331}$ SRP-DF ${ }^{150}$ was used successfully to model the abstraction of $\mathrm{D}$ by $\mathrm{H}$ or $\mathrm{H}$ by $\mathrm{D}$ from $\mathrm{Cu}(111)$ resulting in HD. ${ }^{583}$ Specifically, the DFMDEF calculations using this SRP-DF showed quantitative agreement with experiments ${ }^{666}$ concerning the abstraction cross section, the final rotational state and angular distributions of HD, and the final average $E_{\mathrm{t}}$ of HD. The calculations also showed that modeling ehp excitation was necessary to reach a high level of agreement, which could be explained in terms of energy loss by the incident atom being more important for the hot atom reaction of the lighter $(\mathrm{H})$ incident atom. ${ }^{583}$ Only the final vibrational state distribution was not yet well described by the DFMDEF, which the authors attributed to shortcomings of the QCT method. ${ }^{583}$

It remains to consider the implications of the theory-experiment comparison for $\mathrm{H}_{2}+\mathrm{Cu}(111)$ for the accuracy of GGA-DFT for DC on metals in general. Like initial-state selected reaction probability curves (see Section 4.6), sticking probabilities can be fitted to $\mathrm{S}$-shaped curves characterized by a width (or, inversely, the slope), and an effective barrier height $\left(E_{0}\right)$ or, alternatively, a reaction threshold, where the latter should correspond closely with the minimum barrier height. Correctly describing the sticking probability implies, according to the earlier mentioned hole model, ${ }^{45}$ that the DF used gives a correct description of the reaction threshold or minimum barrier (the onset of the curve) as well as of how the barrier height varies with impact site (energetic corrugation) and molecular orientation, which correlates with the width of the reaction probability curve (see also Section 5.2.1 below). In ref. 177 we showed that several GGA DFs yield very similar results regarding how the barrier height varies with impact site for the activated dissociation of $\mathrm{H}_{2}$ on $\mathrm{Cu}(111)$. Bayesian error statistic applied to DFT likewise suggests that GGA DFs should yield accurate descriptions of how the barrier height varies accross the surface. ${ }^{383}$ As noted earlier, very recent DMC calculations on the highly activated dissociation of $\mathrm{H}_{2}$ on $\mathrm{Al}(110)$ put the idea that GGA (and meta-GGA) DFs yield an accurate description of how the barrier height varies with impact site and molecular orientation on a firm first-principles basis. ${ }^{429}$ However, as we will see later for $\mathrm{H}_{2}+\mathrm{Ru}(0001)$ the description of the width of the reaction probability curve may depend more sensitively on the DF used, and it may require the use of a DF that incorporates van der Waals correlation in at least an approximate way.

5.1.2. $\mathrm{H}_{2}+\mathrm{Cu}(\mathbf{1 0 0})$ and $\mathrm{Cu}(\mathbf{1 1 0})$. The second system for which an SRP-DF was derived was $\mathrm{H}_{2}+\mathrm{Cu}(100) .{ }^{46}$ This turned out to be straightforward: The SRP43 DF for $\mathrm{H}_{2}+\mathrm{Cu}(111)^{43}$ was used to develop a PES for $\mathrm{H}_{2}+\mathrm{Cu}(100)$, and with this PES MB experiments on sticking of $\mathrm{H}_{2}$ to $\mathrm{Cu}(100)$ were described with chemical accuracy. Specifically, the measured $S_{0}{ }^{67}$ were displaced along the energy axis from the spline-interpolated, quantum dynamically calculated $S_{0}$ by less than $43 \mathrm{meV} \approx 1 \mathrm{kcal} \mathrm{mol}^{-1}$ (Fig. 37). ${ }^{46}$ The theory also reproduced the observation ${ }^{667}$ that $S_{0}$ of $\mathrm{H}_{2}$ on $\mathrm{Cu}(111)$ exceeds that of $\mathrm{H}_{2}$ on $\mathrm{Cu}(100)$. All this is encouraging, as it suggests a degree of transferability of SRP-DFs, i.e., that SRP-DFs for a specific molecule interacting with a low index surface of a specific metal may also describe the same molecule interacting with other low index surfaces of that metal with chemical accuracy.

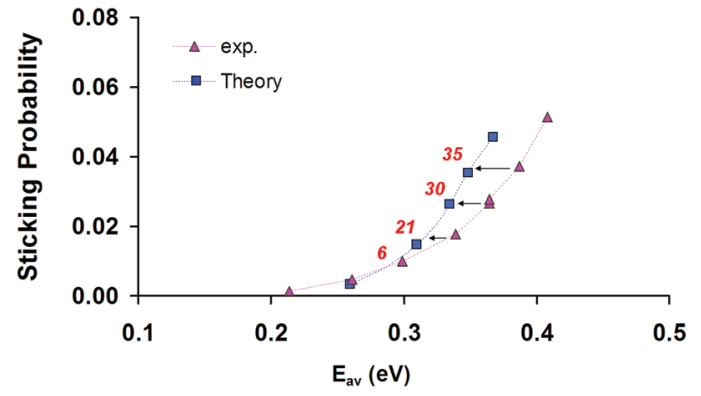

Fig. 37 Measured $^{667}$ and computed ${ }^{46} S_{0}$ are shown as a function of average normal incidence energy for sticking of $\mathrm{H}_{2}$ on $\mathrm{Cu}(100)$. The dynamics calculations were performed ${ }^{46}$ with the TDWP method and used a PES computed with the original SRP DF ${ }^{43}$ derived for $\mathrm{H}_{2}+\mathrm{Cu}(111)$. The numbers shown represent the energy difference in meV between the values of the measured $S_{0}$ and the cubic spline interpolated theoretical $S_{0}$ curve. Reprinted from [L. Sementa, M. Wijzenbroek, B. J. van Kolck, M. F. Somers, A. Al-Halabi, H. F. Busnengo, R. A. Olsen, G. J. Kroes, M. Rutkowski and C. Thewes, et al., Reactive scattering of $\mathrm{H}_{2}$ from $\mathrm{Cu}(100)$ : comparison of dynamics calculations based on the specific reaction parameter approach to density functional theory with experiment, J. Chem. Phys., 2013, 138, 044708], with the permission of AIP Publishing.

The QD calculations also accurately reproduced ${ }^{46}$ measured $^{568}$ probabilities for rotationally (in)elastic scattering of $\mathrm{D}_{2}$ within $v=1$, and measured ${ }^{46} A_{0}^{(2)}(J)$ of $\mathrm{H}_{2}$ desorbing from $\mathrm{Cu}(100)$ in $(v=0, j=1$ and 2$)$ and in $(v=1, j=2-4)$. However, the description obtained for the $A_{0}^{(2)}(J)$ for $(v=0, j=3-5,8)$ and $(v=1, j=1)$ was not yet as accurate, nor were the measured ${ }^{567}$ probabilities for vibrationally elastic and inelastic scattering of $(v=1, j=1) \mathrm{H}_{2}$ from $\mathrm{Cu}(100)$ described accurately. ${ }^{46}$ Finally, the measured ADEs of $(v=0,1, j)$ states were underestimated in the QD calculations using the SRP43 DF (see Fig. 28). However, comparison ${ }^{46}$ to experiments of Comsa and David (which should be accurate as a very long flight path was used in desorption) ${ }^{661}$ again suggests that the problem lies at least partly in the new associative desorption experiments, ${ }^{46}$ which for $\mathrm{Cu}(100)$ may have underestimated the ADEs by about $10 \%$.

Finally, DFMD simulations were also performed ${ }^{173}$ on reaction of $(v=0, j=8)$ and $(v=1, j=4) \mathrm{H}_{2}$ on $\mathrm{Cu}(100)$ using the SRP48 DF developed for $\mathrm{H}_{2}+\mathrm{Cu}(111),{ }^{175}$ which is expected to give the same results as the SRP43 DF developed earlier. ${ }^{43}$ The DFMD calculations yielded lower values of $A_{0}^{(2)}(J)$ for the experimental $T_{\mathrm{S}}$ of $1030 \mathrm{~K}$ than previous calculations with the BOSS model, ${ }^{46}$ as found earlier for $\mathrm{H}_{2}+\mathrm{Cu}(111) \cdot{ }^{175}$ Also, the $R_{v j}\left(E_{i}\right)$ computed with DFMD were shifted to lower energies relative to earlier BOSS results, by 60 and $40 \mathrm{meV}$ for $(v=0, j=8)$ and $(v=1, j=4)$, respectively. ${ }^{173}$ This latter result suggests that the discrepancy between measured and computed ADEs could be even larger for these 2 states as suggested by Fig. 38, which shows results of calculations with the BOSS model. ${ }^{46}$ The study established $\mathrm{H}_{2}+$ $\mathrm{Cu}(100)$ as a useful benchmark system for surface thermal effects on reaction, which should be considerably larger than for $\mathrm{H}_{2}+\mathrm{Cu}(111) .{ }^{173}$

Very recently, an SRP DF was also derived for $\mathrm{H}_{2}+\mathrm{Cu}(110){ }^{467}$ More specifically, Jiang and co-workers ${ }^{467}$ developed PESs for $\mathrm{H}_{2}+\mathrm{Cu}(111), \mathrm{Cu}(100)$, and $\mathrm{Cu}(110)$ based on DFT calculations 


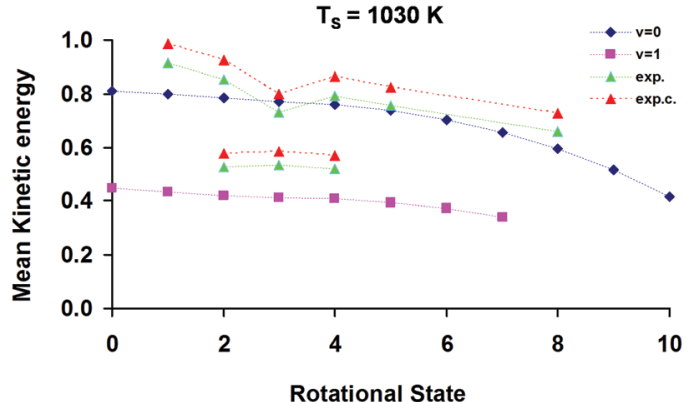

Fig. 38 Calculated 46 (" $v=0$ " and " $v=1$ ") and measured ${ }^{46}$ ("exp") ADEs are shown as a function of the rotational state $\mathrm{J}$ of $\mathrm{H}_{2}$ desorbing from $\mathrm{Cu}(100)$ at $T_{\mathrm{s}}=1030 \mathrm{~K}$. The "exp.c" results were approximately corrected for the use of a static $0 \mathrm{~K}$ surface in the calculations. ${ }^{46}$ Reprinted from [L. Sementa, M. Wijzenbroek, B. J. van Kolck, M. F. Somers, A. Al-Halabi, H. F. Busnengo, R. A. Olsen, G. J. Kroes, M. Rutkowski and C. Thewes, et al., Reactive scattering of $\mathrm{H}_{2}$ from $\mathrm{Cu}(100)$ : comparison of dynamics calculations based on the specific reaction parameter approach to density functional theory with experiment, J. Chem. Phys., 2013, 138, 044708], with the permission of AIP Publishing.

with the optPBE-vdW1 DF. ${ }^{331}$ This functional had previously been shown to yield a chemically accurate description of molecular beam sticking experiments on $\mathrm{D}_{2}+\mathrm{Cu}(111)$ and $\mathrm{H}_{2}+\mathrm{Cu}(100) \cdot{ }^{150}$ Jiang and coworkers trained atomic neural networks for the $\mathrm{H}-\mathrm{H}$, $\mathrm{H}-\mathrm{Cu}$, and $\mathrm{Cu}-\mathrm{Cu}$ interactions to obtain accurately fitted HDNNPs for not only these two systems, but also for $\mathrm{H}_{2}+\mathrm{Cu}(110)$. Next they showed that with the optPBE-vdW1 PESs obtained a chemically accurate description of molecular beam experiments on all three systems can be obtained (see Fig. 39 ) ${ }^{467}$ We will therefore call the optPBE-vdW1 DF an SRP-DF for all three systems, even though this DF was not validated against a second experiment on $\mathrm{H}_{2}+\mathrm{Cu}(100)$ and $\mathrm{H}_{2}+\mathrm{Cu}(110)$. This functional was validated against a second experiment for $\mathrm{H}_{2}+\mathrm{Cu}(111)$ (see Fig. 39A and B), and we regard its also describing the sticking experiments on $\mathrm{H}_{2}+\mathrm{Cu}(100)$ (Fig. 39C) and $\mathrm{Cu}(110)$ (Fig. 39D) without the need for reparametrization as sufficient evidence that this functional allows the minimum barrier height for these systems to be extracted with chemical accuracy. The calculations by Jiang and co-workers suggest ${ }^{467}$ that HDNNPs based on atomic NNs computed with a DF, in such a way that the construction of a chemically accurate HDNNP is enabled for a molecule interacting with one specific low index face of a particular metal, will also yield chemically accurate results for the same molecule interacting with the other low index faces of that metal. This will be the more true if the atomic NNs are trained with DFT calculations on the molecule interacting with all of these low index faces, as was done for $\mathrm{H}_{2}+\mathrm{Cu}^{467}$

5.1.3. $\quad \mathbf{H}_{2}+\mathbf{P t}(111)$. The SRP-DF developed for $\mathrm{H}_{2}+\mathrm{Pt}(111)^{156}$ combined the $\mathrm{PBE} \alpha^{382}$ exchange $\mathrm{DF}$ with the vdW2 correlation $\mathrm{DF}^{327}$ (eqn (2d)), with the fit to the $\mathrm{MB}$ experiment resulting in $\alpha=0.57$. With this DF, QCT calculations were able to reproduce $S_{0}$ measured at normal incidence ${ }^{668}$ with a MD along the energy axis between theory and the spline fitted experimental $S_{0}$ curve of just $0.25 \mathrm{kcal} \mathrm{mol}^{-1}$ (see also Fig. 5 of ref. 156). Because the theory also reproduced experiment for off-normal incidence polar angles of 30 and $45^{\circ}$ (see Fig. 40) while the reaction does not obey normal energy scaling, the DF tested could be called
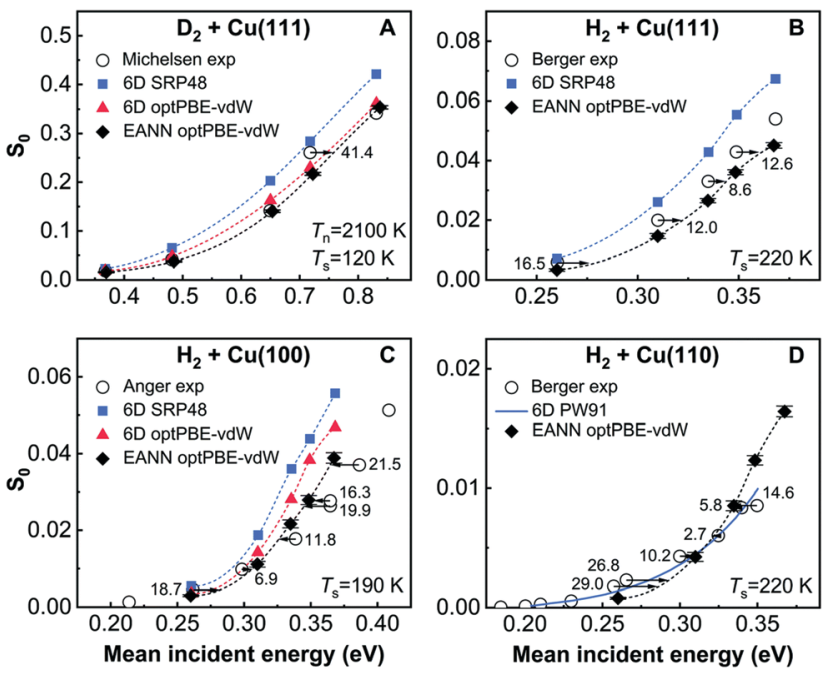

Fig. $39 S_{0}$ computed with PESs based on the optPBE-vdW1 DF for $D_{2}+$ $\mathrm{Cu}(111)(\mathrm{A}), \mathrm{H}_{2}+\mathrm{Cu}(111)$ (B), $\mathrm{H}_{2}+\mathrm{Cu}(100)$, and $\mathrm{H}_{2}+\mathrm{Cu}(110)$ with high-dimensional QCT calculations are compared with molecular beam sticking experiments ${ }^{12,649,667,730}$ on these systems. Black arrows and accompanying numbers (in $\mathrm{meV}$ ) indicate the incident energy spacing between the experimental and interpolated computed curves. Reproduced from ref. 467 with permission from the PCCP Owner Societies.

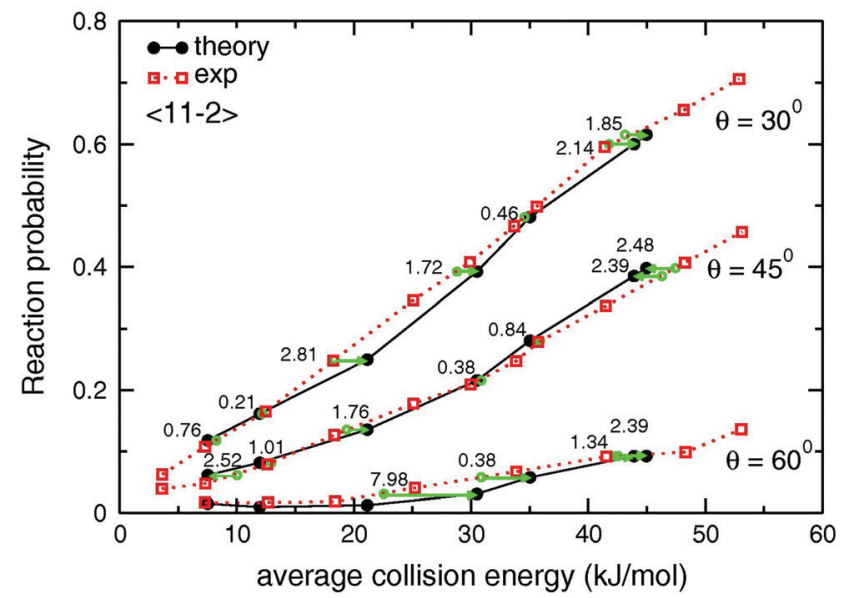

Fig. $40 S_{0}$ measured ${ }^{668}$ and computed ${ }^{156}$ with the SRP density functional for $D_{2}+P t(111)$ are shown as a function of the average incidence energy for off-normal incidence at $\theta_{i}=30^{\circ}, 45^{\circ}$, and $60^{\circ}$. In the calculations incidence is along the $\langle 11-2\rangle$ direction. The errors and numbers (in $\mathrm{kJ} \mathrm{mol}^{-1}$ ) show the collision energy spacing between the computed values and the interpolated experimental $S_{0}$ values (shown by the green circles). Reprinted from E. N. Ghassemi, M. Wijzenbroek, M. F. Somers and G. J. Kroes, Chemically accurate simulation of dissociative chemisorption of $D_{2}$ on Pt(111), Chem. Phys. Lett., 2017, 683, 329-335, licensed under CC-BY 4.0.

an SRP-DF. ${ }^{156}$ Another DF that is an SRP-DF for $\mathrm{H}_{2}+\mathrm{Pt}(111)$ is the B86SRP68-DF2 functional, which also is an SRP-DF for $\mathrm{H}_{2}+$ $\mathrm{Cu}(111){ }^{658}$

A problem noted for $\mathrm{H}_{2}+\operatorname{Pt}(111)$ is that with the SRP PBE $\alpha$-vdW2 DF the diffraction of $\mathrm{H}_{2}$ scattering from $\mathrm{Pt}(111)$ is not yet well described. ${ }^{163}$ For example, for scattering with incidence along the $\langle 10 \overline{1}\rangle$ incidence direction, the specular scattering probability is 

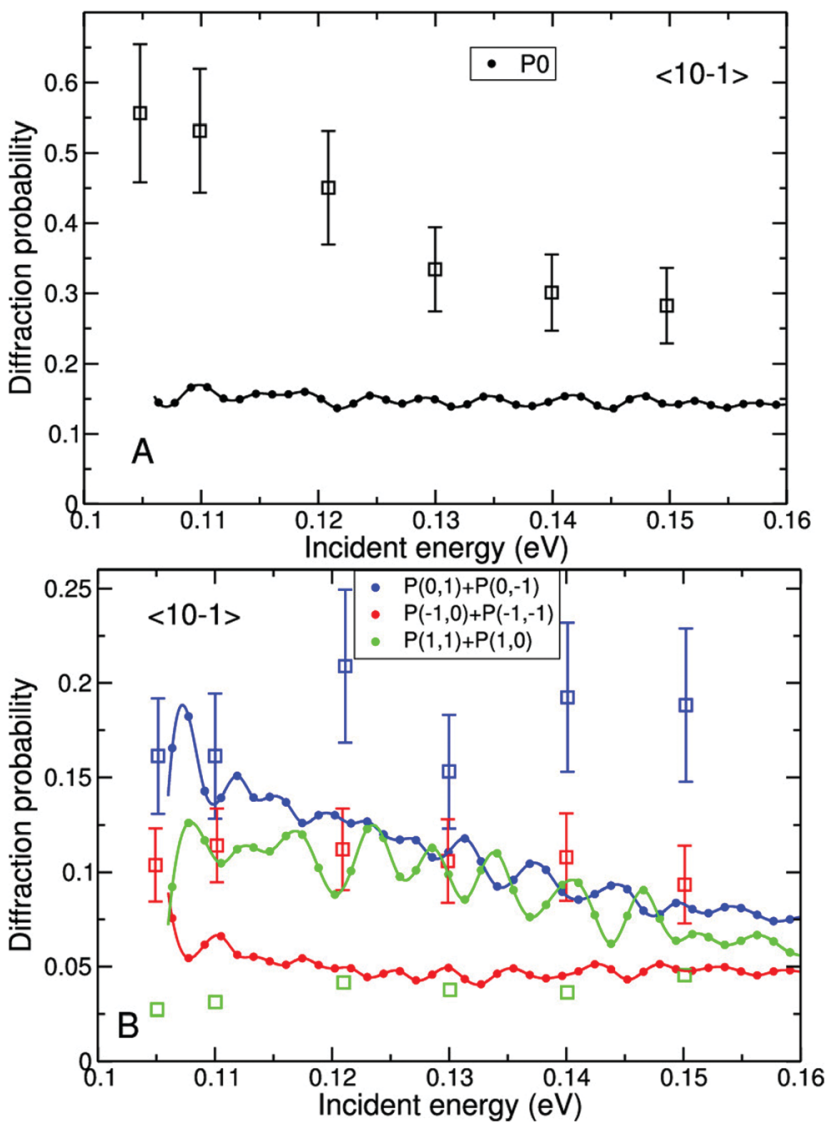

Fig. 41 Measured ${ }^{615}$ diffraction probabilities (squares, with error bars where available) are compared with values computed ${ }^{163}$ (filled circles and interpolating curves) with QD and SRP-DFT for $\mathrm{H}_{2}$ scattering from $\mathrm{Pt}(111)$, for specular scattering (A) and for first order diffraction (B). In all cases, incidence is along the $\langle 10-1\rangle$ direction. Probabilities for symmetry equivalent transitions have been summed. Figure taken from ref. 163 (https://pubs.acs. org/doi/10.1021/acs.jpcc.9b00981). Further permission requests to be directed to the ACS.

grossly underestimated in the theory (Fig. 41A). ${ }^{163}$ Also, the order in the sums of almost symmetry equivalent out-of-plane diffraction probabilities is incorrectly described (Fig. 41B). ${ }^{163}$ This came as a surprise, as previously ${ }^{615}$ semi-quantitative agreement was obtained with both the experiments on reaction shown in Fig. 40 and the diffraction experiments shown in Fig. 41 in QD calculations based on a GGA DF, i.e., the B88P86 ${ }^{313,669}$ DF. However, a similar problem was noted for $\mathrm{H}_{2}+\mathrm{Ru}(0001)$ with the use of c-SRP DFs containing van der Waals correlation DFs ${ }^{151}$ (see also below). Furthermore, MCTDH calculations on He diffraction from $\mathrm{Ru}(0001)$ with the GGA PBE DF agreed better with experiment than approaches approximately including the van der Waals interaction. ${ }^{670}$ One explanation ${ }^{163}$ holds that DW extrapolation to $0 \mathrm{~K}$ constitutes an incorrect procedure for obtaining experimental $0 \mathrm{~K}$ results that can be compared with static surface theoretical results in the presence of a van der Waals well that can give rise to indirect scattering, as DW extrapolation should only be valid for direct scattering. Better (though still semi-quantitative) agreement of $\mathrm{H}_{2}$ diffraction experiments with dynamics calculations may be obtainable with GGA PESs, ${ }^{615}$ as the resulting theoretical dynamics might likewise only reflect direct scattering, as the van der Waals well is missing.

SRP-DFs can also be used to understand differences between experiments, as also illustrated for highly activated dissociation where differences between $S_{0}$ due to different beam conditions can be dramatic (see Fig. 31 and its discussion above). Differences between $S_{0}$ for the weakly activated dissociation of $\mathrm{D}_{2}$ on $\mathrm{Pt}(111)$ measured by different groups can also be substantial, as shown in Fig. 42 for three different MB experiments. ${ }^{193,668,671}$ QCT calculations based on the derived SRP-DF ${ }^{156}$ tested whether it is plausible that the large differences shown in Fig. 42 could be due to using different $\mathrm{D}_{2}$ beams, employing appropriate parameter sets describing the beams. The calculations suggested that the results should be almost identical for $E_{i} \leq 0.35 \mathrm{eV}$, regardless of whether parameters are used describing seeded and translationally broad (SBG or SBC) beams, or pure and translationally narrow beams (PNH or PNA) (Fig. 43). ${ }^{163}$ Two sets of experiments ${ }^{193,668}$ could be reproduced to within chemical accuracy using appropriate sets of beam parameters. ${ }^{163}$ This suggests that differences in the beam parameters are not the cause of the discrepancies observed in Fig. 42. A thorough analysis of the results and the experiments suggests that the $S_{0}$ measured in the other experiment ${ }^{671}$ were underestimated by $10-15 \%$. This presumably happened because the yet to be normalized $S_{0}$ obtained from thermal desorption were incorrectly calibrated with one or a few King and Wells measurements carried out for high $E_{i \cdot}{ }^{163}$

5.1.4. $\mathbf{C H}_{4}+\mathbf{N i ( 1 1 1 )}$. The SRP-DF developed for $\mathrm{CHD}_{3}+$ $\mathrm{Ni}(111)^{44}$ used an exchange DF that is a weighted average of the $\operatorname{RPBE}^{672} \mathrm{DF}(32 \%, \alpha=0.32)$ and the $\mathrm{PBE}^{195} \mathrm{DF}(68 \%)$, combined with the vdW1 correlation $\mathrm{DF}^{326}$ (eqn (2c)). The $\alpha$-parameter was fitted to two laser-off experiments performed for $T_{\mathrm{N}}=600$ and $650 \mathrm{~K}\left(E_{i}=112.3\right.$ and $\left.121.2 \mathrm{~kJ} \mathrm{~mol}^{-1}\right)$ using DFMD calculations. This resulted in excellent agreement with experiment for

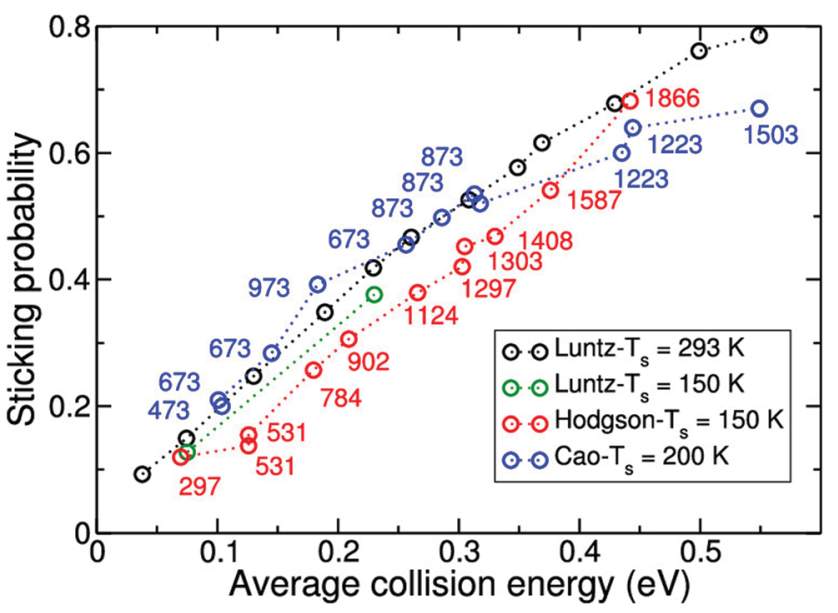

Fig. $42 S_{0}$ as measured for $D_{2}+P t(111)$ by Hodgson and coworkers ${ }^{671}$ (red circles), Luntz et al. ${ }^{668}$ (black circles for $T_{\mathrm{s}}=293 \mathrm{~K}$, green circles for $T_{\mathrm{s}} \approx 150 \mathrm{~K}$, and Cao et al. ${ }^{193}$ (blue circles)). The values of the $T_{\mathrm{n}}$ used in the experiments are indicated (in K) for the experiments of Hodgson and coworkers ${ }^{671}$ and Cao et al. ${ }^{193}$ Figure taken from ref. 163 (https://pubs.acs. org/doi/10.1021/acs.jpcc.9b00981). Further permission requests to be directed to the ACS. 


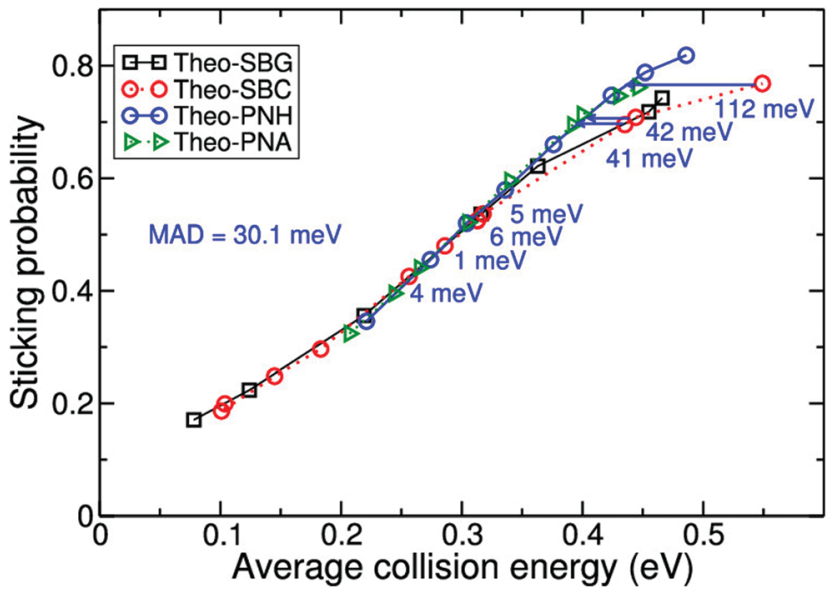

Fig. 43 Comparison of $S_{0}$ computed for $D_{2}+P t(111)$ with the SRP DF for this systems, for four sets of molecular beam parameters ${ }^{163}$ characterizing different kinds of $D_{2}$ molecular beams, i.e., seeded beams that are comparatively broad in translational energy $\left(\mathrm{SBG}^{200}\right.$ and $\left.\mathrm{SBC}^{193}\right)$, and pure $\mathrm{D}_{2}$ beams that are comparatively narrow in translational energy $\left(\mathrm{PNH}^{186,671}\right.$ and $\mathrm{PNA}^{731}$ ). Arrows and numbers show the incidence energy differences between results obtained with the SBC parameters and interpolated values of the results obtained with the PNH parameters. Figure taken from ref. 163 (https://pubs.acs.org/doi/10.1021/acs.jpcc.9b00981). Further permission requests to be directed to the ACS.

laser-off reaction at $T_{\mathrm{N}}=550 \mathrm{~K}\left(E_{i}=101.1 \mathrm{~kJ} \mathrm{~mol}^{-1}\right.$, Fig. 44A). The DFMD calculations resulted in too high laser-off $S_{0}$ for higher $T_{\mathrm{N}}$ and $E_{i}$, but this could be attributed to D-atom bending excited vibrational states present in the $\mathrm{MB}$ at the higher $T_{\mathrm{N}}$ required $(>650 \mathrm{~K}) .{ }^{44}$ On the other hand, the DFMD calculations resulted in $S_{0}$ in excellent agreement with experiment for reaction of $\mathrm{CHD}_{3}$ excited with one quantum in the $\mathrm{CH}_{-}$ stretch $\left(\nu_{1}\right)$ mode (Fig. 44A). For this reason, the DF developed (henceforth called SRP32-vdW1) could be called an SRP-DF. Earlier calculation $\mathrm{s}^{157}$ had suggested that experimental data for $\mathrm{CHD}_{3}+\mathrm{Pt}(111)$ could not be reproduced with a simple mixed PBE-RPBE exchange DF combined with PBE correlation (as in eqn (2b)), while better results were obtained with a mixed PBE-RPBE exchange DF combined with vdW1 correlation. This explains the choice of an SRP-DF as expressed in eqn (2c).

The SRP-DF could also be used to obtain useful mechanistic insights. DFMD calculations performed on $\mathrm{CHD}_{3}+\mathrm{Ni}(111)$ and $\mathrm{CHD}_{3}+\mathrm{Pt}(111)$ for $E_{i}$ corresponding to roughly the same $S_{0}$ (i.e., $\approx 0.03)$ suggest that on $\operatorname{Pt}(111)$ the molecules react closer to the top sites (Fig. 45A and B). ${ }^{44}$ This suggests that in QD calculations for $\mathrm{CHD}_{3}$ on $\mathrm{Pt}(111)$ an approximation which neglects the dependence of the PES on the azimuthal $\varphi$ angle ${ }^{60}$ (Fig. 25) should work much better than on Ni(111), as the PES only exhibits a weak dependence on this angle at and near the top site for methane-metal surface systems. ${ }^{60,456}$ Furthermore, for the reacting molecules the initial distribution of the angle $\beta$ the remaining methyl principle axis makes with the surface normal is quite close to the distribution at the time of reaction (Fig. 45C). In line with similar DFMD and RPH results for $\mathrm{CH}_{4}+\mathrm{Ni}(111)^{89}$ (Section 4.4.4), this finding suggests that the RSA should work better than a RAA. The latter approximation

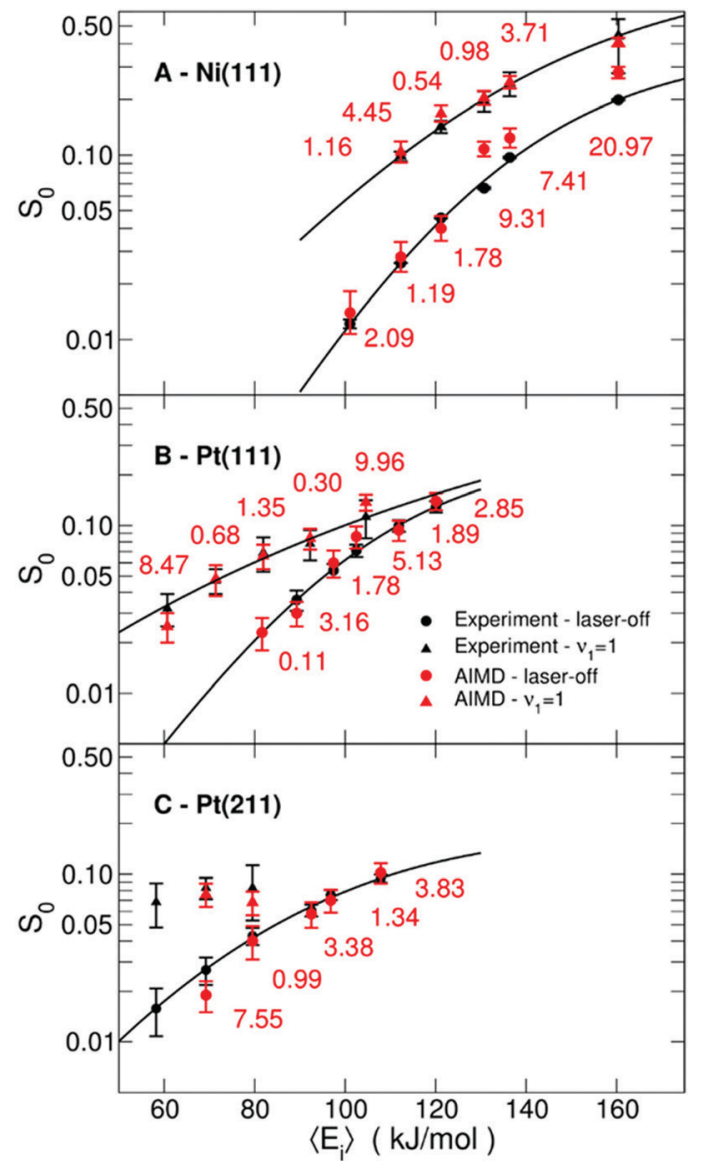

Fig. $44 S_{0}$ computed with DFMD using the SRP32-vdW DF for (A) $\mathrm{CHD}_{3}+$ $\mathrm{Ni}(111),{ }^{44}$ (B) $\mathrm{Pt}(111),{ }^{47}$ and (C) $\mathrm{Pt}(211)^{47}$ are compared with measured values, ${ }^{44,47}$ for laser-off reaction and for the $\nu_{1}=1 \mathrm{CH}$-stretch excited state. Numbers show the displacement along the incidence energy axis of the computed reaction probabilities relative to the fitted experimental $\mathrm{S}_{0}$-curve, in $\mathrm{kJ} \mathrm{mol}^{-1}$. Reprinted from ref. 47 (https://pubs.acs.org/doi/10. 1021/acs.jpclett.7b01905). Further permission requests to be directed to the ACS.

would imply the initial distribution of this angle to equal the sine distribution displayed in Fig. 45C for the reacting molecules, which is clearly not the case.

Very recently, the SRP32-vdW1 DF has been used in RPH calculations on vibrationally inelastic scattering of $\left(\nu_{3}=1\right) \mathrm{CH}_{4}$ from $\mathrm{Ni}(111)$ and $\mathrm{Ni}(111)$ covered by a monolayer of graphene $(\mathrm{Gr} / \mathrm{Ni}(111)){ }^{673}$ In agreement with recent experiments, ${ }^{240}$ the calculations revealed substantial vibrationally inelastic scattering to the $\left(\nu_{1}=1\right)$ symmetric stretching state (also called surface induced IVR). ${ }^{673}$ Again in agreement with experiment, ${ }^{240}$ hardly any vibrationally inelastic scattering to excited bending states was observed, and hardly any vibrationally inelastic scattering was found in scattering from $\mathrm{Gr} / \mathrm{Ni}(111) .{ }^{673}$ The calculations showed a strong dependence of the vibrationally inelastic scattering probability on impact site, with a high excitation probability correlating with a high catalytic activity of the site. ${ }^{673}$ The calculations were performed for a static surface ${ }^{673} \mathrm{~A}$ single calculation performed for a distorted $\mathrm{Ni}(111)$ lattice suggests a strong influence of lattice motion. ${ }^{673}$ The results suggest that 

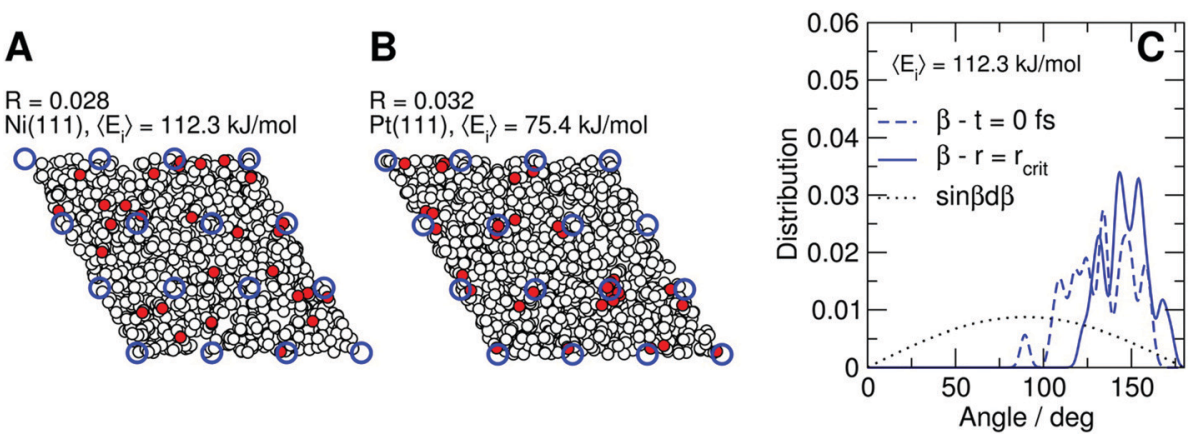

Fig. 45 Impact sites and molecular orientation in collisions of $\mathrm{CHD}_{3}$ with $\mathrm{Ni}(111)$ and $\mathrm{Pt}(111)$. (A and $\left.\mathrm{B}\right)$ Initial distribution of impact sites for reacting (red circles) and scattering (white circles) molecules above Ni(111) at $E_{i}=112.3 \mathrm{~kJ} \mathrm{~mol}^{-144}(\mathrm{~A})$ and Pt(111) at $E_{i}=75.4 \mathrm{~kJ} \mathrm{~mol}^{-151}$ (B). Blue circles indicate first layer atoms in ideal positions. (C) Molecular orientation of reactive trajectories for $\mathrm{CHD}_{3}+\mathrm{Ni}(111)$ at $E_{i}=112.3 \mathrm{~kJ} \mathrm{~mol}^{-144}$, showing the angle the principal axis of the methyl fragment (which points to the $\mathrm{C}$-atom), $\beta$, makes with the surface normal (blue traces). Dashed (solid) traces represent the orientation at time zero (at the time of reaction). The dotted line illustrates random uniform sampling. Reprinted from ref. 44 (https://pubs.acs.org/doi/10.1021/acs. jpclett.6b01022). Further permission requests to be directed to the ACS

averaging dynamics results of explicit dynamics calculations performed for different lattice configurations, as has been done with the PSA for $\mathrm{H}_{2}+\mathrm{Cu}(111),{ }^{171}$ will be required to obtain accurate results for vibrationally inelastic scattering. The authors also mention the desirability of a better treatment of rotation and perhaps even parallel translation for obtaining more accurate results. ${ }^{673}$

The SRP32-vdW1 DF has been used to develop a 15D PES for $\mathrm{CH}_{4}+\mathrm{Ni}(111)$, based on the ${ }^{167}$ PIP-NN method. $S_{0}$ computed with the QCT method and corrected a posteriori with the LRS method $^{167}$ compared well with the previous DFMD results ${ }^{44}$ regardless of whether the Einstein or the Debye model was used to describe surface atom motion. The only exception occurred in the simulation of MB laser-off experiments at low $E_{i}$, where the Einstein model resulted in more accurate results, although the surface atom vibrations should be best described with the Debye model. A problem here may be that the electronic and mechanical coupling parameters are computed with DFT calculations appropriate for the Einstein model (considering the motion of a single surface atom and keeping the other atoms fixed at their ideal lattice positions). ${ }^{167}$

The 15D PES just discussed has also been used in QCT calculations ${ }^{161}$ simulating He-seeded MB experiments on sticking of $\mathrm{CHD}_{3}$ to $\mathrm{Ni}(111)$ at $E_{i}<100 \mathrm{~kJ} \mathrm{~mol}^{-1}$ (Fig. 19). As discussed in Section 4.2, the overestimation of the measured $S_{0}$ should at least partly be due to the attempt to simulate the reaction at too high $T_{\mathrm{N}}$ (>650 K). Zhou and Jiang also computed $\mathrm{CH} / \mathrm{CD}$ bond breaking ratios that were too low (about 20\%) compared to experimental values (40-25\%). Again we expect that this is due to the attempt to simulate the reaction at too high $T_{\mathrm{N}}$ where CD-vibrational modes become too much excited for the classical approximation to be valid, due to artificial leaking of vibrational energy among modes occurring already in the gas phase.

Finally, the 15D PES was also used to test the novel MGLO method ${ }^{153}$ against DFMD results for $\mathrm{CHD}_{3}+\mathrm{Ni}(111) \cdot{ }^{44}$ As noted above the outcome was quite promising, with the MGLO results being in very good agreement with the DFMD results. ${ }^{153}$

5.1.5. $\mathbf{C H}_{4}+\operatorname{Pt}(\mathbf{1 1 1})$, Pt(211). The SRP-DF developed for $\mathrm{CHD}_{3}+\mathrm{Ni}(111)^{44}$ (the SRP32-vdW1 DF) also turned out to be an SRP-DF for $\mathrm{CHD}_{3}+\mathrm{Pt}(111) \cdot{ }^{47}$ DFMD calculations using this DF described both laser-off reaction $\left(\mathrm{MD}=0.59 \mathrm{kcal} \mathrm{mol}^{-1}\right)$ and $\nu_{1}=1$ reaction $\left(\mathrm{MD}=0.99 \mathrm{kcal} \mathrm{mol}^{-1}\right)$ with chemical accuracy (Fig. 44B). The MD is close to the limit of chemical accuracy for $\nu_{1}=1 \mathrm{CHD}_{3}$, but note that the largest errors occur where the distance between the computed $S_{0}$ to the extrapolated experimental $S_{0}$ fit had to be determined while extrapolating the fit. Laser-off reaction could be described accurately because the $T_{\mathrm{N}}$ used to expand the MB could be kept at values $\leq 650 \mathrm{~K}$ for the entire range of $E_{i}$ probed, as the $E_{\mathrm{b}}$ for DC of $\mathrm{CHD}_{3}$ on Pt(111) $\left(78.7 \mathrm{~kJ} \mathrm{~mol}^{-1}\right)^{47}$ is lower than on $\mathrm{Ni}(111)\left(97.9 \mathrm{~kJ} \mathrm{~mol}^{-144}\right)$. The calculations on $\mathrm{CHD}_{3}+\mathrm{Ni}(111)$ and $\mathrm{Pt}(111)$ suggest that a SRP-DF for a specific molecule interacting with a particular low index face of a metal belonging to a specific group may be transferable to the same molecule interacting with the same low index face of a different metal belonging to the same group.

The SRP32-vdW1 $\mathrm{DF}^{44}$ was also used in DFMD calculations on $\mathrm{CHD}_{3}+\mathrm{Pt}(111)$ comparing to DFMD simulations with the $\mathrm{PBE}^{195} \mathrm{DF}^{84}$ The aim was to explain why DFMD simulations with the SRP32-vdW1 DF agreed with laser-off experiments to within chemical accuracy while the DFMD calculations using the PBE DF substantially overestimated the laser-off $S_{0}$, even though both DFs yield very similar $E_{\mathrm{b}}$. The calculations suggest that the difference comes from the van der Waals well present with SRP32-vdW1 but absent with PBE. The well leads to acceleration of methane travelling towards the barrier (and therefore to less time for dynamical readjustments), which also leads to more energy transfer to the surface atoms. ${ }^{84}$

The SRP-DF for $\mathrm{CHD}_{3}+\mathrm{Pt}(111)$ (the SRP32-vdW DF) also is an SRP-DF for $\mathrm{CHD}_{3}+\mathrm{Pt}(211) .{ }^{47}$ DFMD calculations using this DF described laser-off reaction $\left(\mathrm{MD}=0.82 \mathrm{kcal} \mathrm{mol}^{-1}\right.$ ) with chemical accuracy (Fig. 44C). The measured $S_{0}$ curve for $\nu_{1}=1 \mathrm{CHD}_{3}$ is too flat to determine a MD by drawing lines from computed $S_{0}$ to the fitted experimental $S_{0}$ curve, but the computed $S_{0}$ all fall within the error bars of the measured $S_{0}$. No computed $S_{0}$ could be determined for the lowest $E_{i}$ studied due to the importance of trapping at this $E_{i}$. Importantly, the result obtained suggests that a SRP-DF for a specific molecule interacting with a low index face of a particular metal may be transferable to the same molecule interacting with a stepped surface of that metal. 


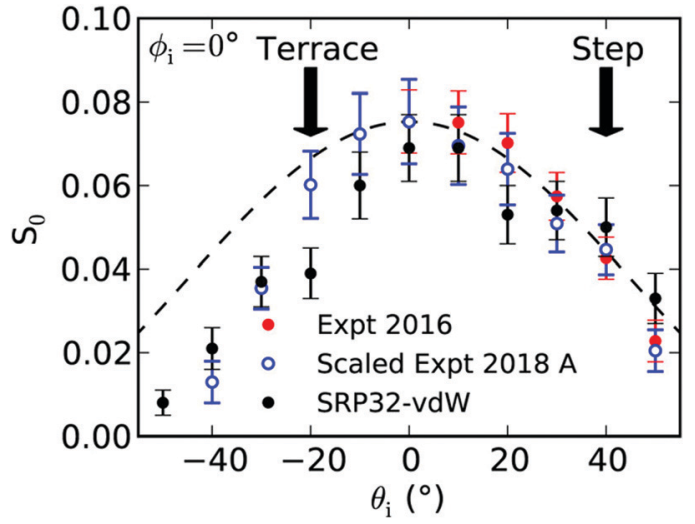

Fig. 46 Comparison of the sticking coefficients computed for $\mathrm{CHD}_{3}+$ Pt(211) with DFMD using the SRP32-vdW DF (black circles) with experimental values at an incident energy of $96.8 \mathrm{~kJ} \mathrm{~mol}^{-1}$ (red circles), and with appropriately scaled sticking coefficients from experiments at an incident energy of $98.5 \mathrm{~kJ} \mathrm{~mol}^{-1}$ (blue open circles). The results are shown as a function of the polar angle of incidence, $\theta_{i}$, with the incidence plane taken perpendicular to the (100) steps. The dashed black line shows a $\cos \left(2 \theta_{i}\right)$ distribution, and the arrows denote the $\theta_{i}$ values corresponding to incidence perpendicular to the (100) step and to the (111) terrace, respectively. Reprinted from ref. 165 (https://pubs.acs.org/doi/10.1021/acs.jpcc.8b05887). Further permission requests to be directed to the ACS.

The SRP-DF for $\mathrm{CHD}_{3}+\mathrm{Pt}(211)$ was tested in calculations and experiments on sticking at off-normal incidence, ${ }^{165}$ with the incidence plane perpendicular to the steps. The computed $S_{0}\left(\theta_{i}\right)$ were in excellent agreement with the measured values (Fig. 46). ${ }^{165}$ Also, an asymmetry was seen, with $S_{0}\left(\theta_{i}=40^{\circ}\right)$ being much larger than $S_{0}\left(\theta_{i}=-40^{\circ}\right)$ in both the calculations and the experiment, with $\theta_{i} \approx 40^{\circ}$ defining incidence perpendicular to the step (Fig. 46). The computed $S_{0}\left(\theta_{i}\right)$ for incidence in the plane parallel to the step were symmetric in $\theta_{i}{ }^{165}$

The SRP32-vdW1 $\mathrm{DF}^{44}$ was also used in DFMD calculations comparing the dissociation dynamics of $\mathrm{CHD}_{3}$ on $\mathrm{Pt}(111)$ and $\operatorname{Pt}(211) .{ }^{164}$ In spite of differences between the barrier heights, the geometry of the TSs and many aspects of the dissociation dynamics were very similar for both surfaces, with the only exceptions being that trapping and energy transfer to surface atom motion were enhanced on the stepped (211) surface. ${ }^{164}$

Finally, the SRP32-vdW1 $\mathrm{DF}^{44}$ was also used in $\mathrm{RPH}$ calculations $^{162}$ comparing with MB sticking experiments using resonance enhanced infrared spectroscopy (RAIRS) detection of $\mathrm{CH}_{\mathrm{n}}$ fragments on $\mathrm{Pt}(211){ }^{162}$ Using RAIRS the experiments measured probabilities for sticking of $\mathrm{CH}_{4}$ to the steps of $\operatorname{Pt}(211) .{ }^{162}$ Computed laser-off and $\nu_{3}=1$ probabilities for sticking to the steps were in excellent agreement with experiment (see Fig. 5A of ref. 162). The agreement with sticking to the terraces was less good, but this may have been due to the analysis of the RAIRS intensities for sticking to the (111) terraces of Pt(211) using RAIRS conversion factors for the flat Pt(111) surface. ${ }^{162}$

\subsection{Systems for which candidate SRP-DFs have been derived}

5.2.1. $\quad \mathbf{H}_{2}+\mathbf{R u}(\mathbf{0 0 0 1})$. Research on $\mathrm{H}_{2}+\mathrm{Ru}(0001)$ showed that the strategy used to develop an SRP-DF for $\mathrm{H}_{2}+\mathrm{Cu}(111)$, i.e., to take a weighted average of the semi-local RPBE and PBE

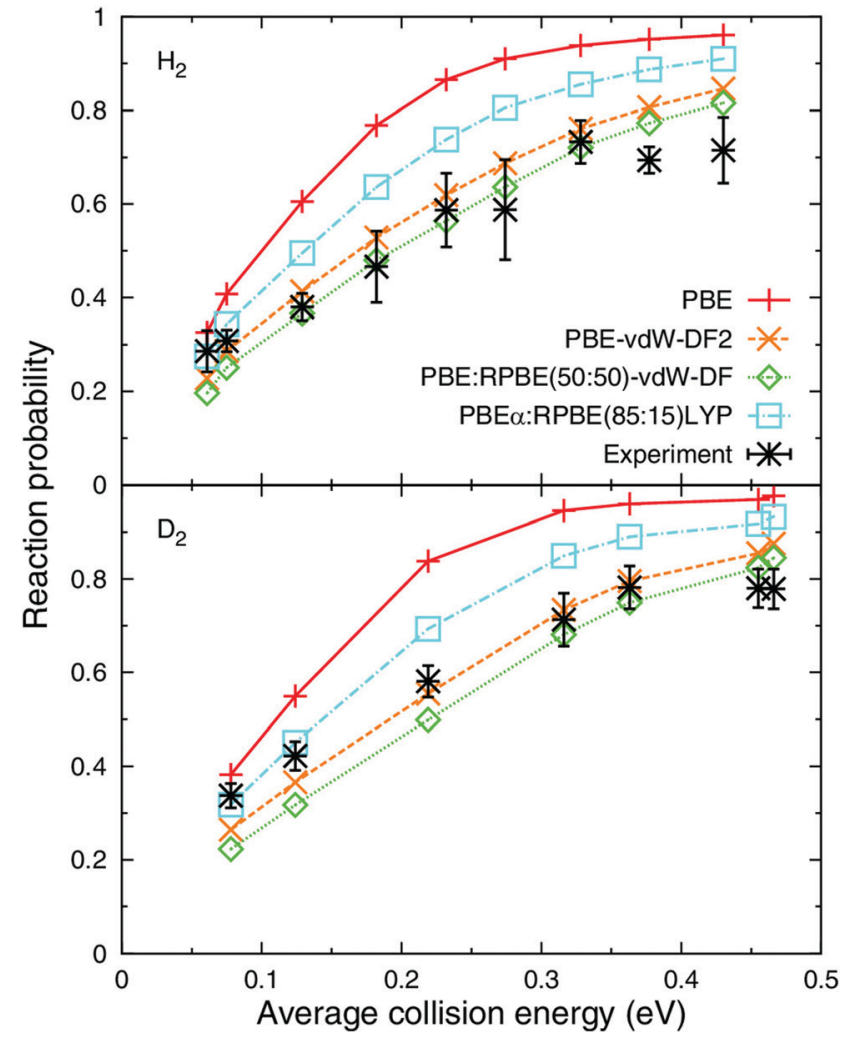

Fig. $47 S_{0}$ computed $^{151}$ with two c-SRP-DFs (PBE-vdW-DF2 and PBE: $\operatorname{RPBE}(50: 50)$-vdW-DF1), with the PBE DF, and with the other DF indicated are compared with experimental values ${ }^{200}$ for $\mathrm{H}_{2}$ (upper panel) and $\mathrm{D}_{2}$ (lower panel) scattering from Ru(0001). Reprinted from [M. Wijzenbroek and G. J. Kroes, The effect of the exchange-correlation functional on $\mathrm{H}_{2}$ dissociation on Ru(0001), J. Chem. Phys., 2014, 140, 084702], with the permission of AIP Publishing.

(or its predecessor PW91) DFs as in eqn (2b) (or eqn (2a)), does not work for the former reaction. The $S_{0}$ computed with PBE and $\mathrm{RPBE}^{151}$ do not straddle the measured $S_{0}{ }^{200}$ (Fig. 1): with $\mathrm{PBE}(\mathrm{RPBE})$ reaction is well described (underestimated) at low $E_{i}$, and overestimated (well described) at high $E_{i}$. Recognizing that the van der Waals interaction might well affect this early barrier reaction, NL DFs consisting of mixtures of $\mathrm{PBE}^{195}$ and $\mathrm{RPBE}^{196}$ exchange and $\mathrm{vdW} 1^{326}$ or $\mathrm{vdW} 2^{327}$ correlation were tested. ${ }^{151}$ The PBE-vdW2 and the PBE:RPBE(50:50)-vdW1 DFs gave an excellent description of the sticking of $\mathrm{H}_{2}$ and $\mathrm{D}_{2}$ (Fig. 47). ${ }^{151} \mathrm{~A}$ lesser point is that the use of the "workhorse" mGGA revTPSS DF ${ }^{199}$ leads to somewhat improved comparison with experiment when compared with the general purpose PBE GGA DF (Fig. 1).

An attempt to validate these DFs as true SRP-DFs failed, ${ }^{151}$ as QD failed to reproduce measured ${ }^{116,674}$ diffraction probabilities for $\mathrm{H}_{2}+\mathrm{Ru}(0001)$, which were overestimated by a factor up to 2 for specular scattering and factors up to 3 for in-plane and out-ofplane first order diffraction. The results were found to be somewhat worse than results obtained with a standard GGA DF (PW91). ${ }^{151}$ Due to the failure to accurately describe the additional (diffraction) experiment, the two DFs found to accurately describe sticking can be called c-SRP DFs, but not SRP-DFs, as the experiments on sticking of $\mathrm{H}_{2}$ and $\mathrm{D}_{2}$ do not show important isotope 
effects that can be used for validation purposes. The problem with the diffraction can to a large extent (for seven out of the nine diffraction channels measured) be fixed by assuming a particular kind of static surface disorder in the dynamics calculations, which can be characterized through a single parameter. ${ }^{148}$ However, the presence of this type of disorder in the experiments on $\mathrm{H}_{2}+$ $\mathrm{Ru}(0001)$ diffraction has yet to be established.

The brute force search for SRP-DFs also pointed to a strategy for discovering good SRP-DFs ${ }^{151}$ that is in accordance with the earlier mentioned hole model. ${ }^{45}$ Experimental $S_{0}$ curves can be characterized by a reaction threshold (energy at reaction onset) and a width (how fast $S_{0}$ increases with $E_{i}, e . g$. the $W$-parameter in eqn (32) and (33)). The threshold and the width correlate with the minimum $E_{\mathrm{b}}$ ( $x$-axis of Fig. 48) and the energetic corrugation of the $E_{\mathrm{b}}$ (how $E_{\mathrm{b}}$ varies across the surface, y-axis of Fig. 48). The results for $\mathrm{H}_{2}+\mathrm{Ru}(0001)$ suggest that c-SRP DFs should be found in a narrow region in a plot of the minimum $E_{\mathrm{b}}$ and a measure of the energetic corrugation (difference between minimum barrier and barrier at other site), as indicated by the red circle in Fig. 48. ${ }^{151}$ This can guide the search for SRP-DFs, also noting that DFs employing similar correlation DFs produce results lying approximately on a straight line in figures like Fig. 48.

5.2.2. $\mathbf{H}_{2}+\mathbf{N i ( 1 1 1 )}$. A c-SRP DF has also been derived for $\mathrm{H}_{2}+\mathrm{Ni}(111) .{ }^{476} S_{0}$ computed using a PES computed with the PBE-vdW2 DF (green triangles in Fig. 49), reproduced experimental results of Resch et $a{ }^{675}$ (black crosses Fig. 49) with chemical accuracy: The MD between the measured $S_{0}$ and the interpolated computed $S_{0}$ was $0.6 \mathrm{kcal} \mathrm{mol} \mathrm{m}^{-1}{ }^{476}$ Fig. 49 illustrates two

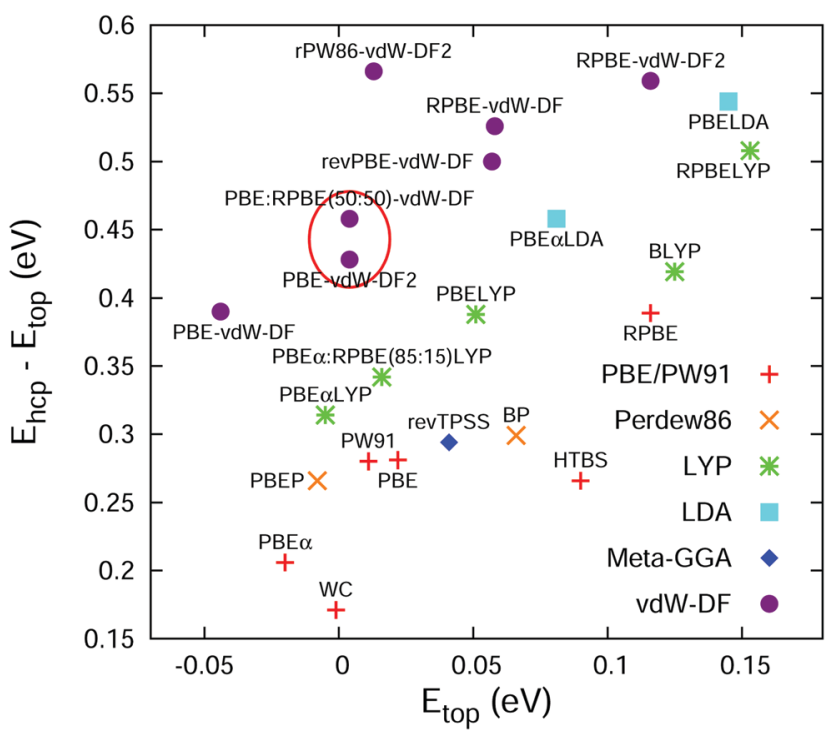

Fig. 48 The energetic corrugation of the potential is shown as a function of the lowest barrier height on the basis of potential energy surfaces constructed for $\mathrm{H}_{2}+\mathrm{Ru}(0001)$ using the DFs indicated. The DFs are grouped (symbols) by the correlation functional used. For the references of the DFs, see Table 4 of ref. 151. The red circle indicates the DFs that are C-SRP-DFs for $\mathrm{H}_{2}+\mathrm{Ru}(0001)$. Reprinted from [M. Wijzenbroek and G. J. Kroes, The effect of the exchange-correlation functional on $\mathrm{H}_{2}$ dissociation on Ru(0001), J. Chem. Phys., 2014, 140, 084702], with the permission of AIP Publishing.

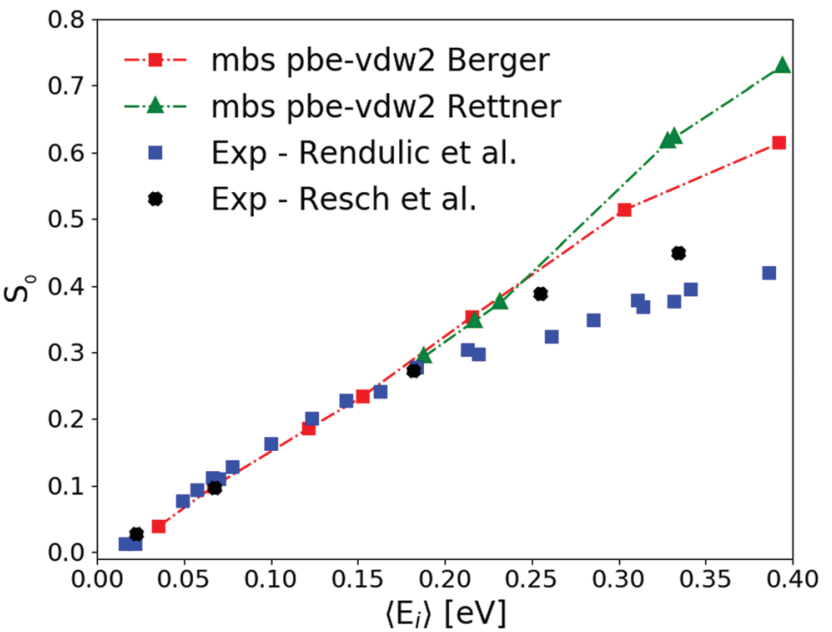

Fig. 49 Comparison of $\mathrm{S}_{0}$ computed for $\mathrm{H}_{2}+\mathrm{Ni}(111)$ with the PBE-vdW2 DF using molecular beam parameters fitted to ${ }^{476}$ TOF-distributions published in ref. 648 (red square box) and parameters describing the $\mathrm{H}_{2}$ beams used by Rettner et al. ${ }^{641}$ (green upper triangle), with values measured in experiments ${ }^{642,675}$ for $\mathrm{H}_{2}$ incident normal to $\mathrm{Ni}(111)$. Data taken from ref. 476.

difficulties often encountered when attempting to fit SRP-DFs: (i) the beam parameters had to be guessed, and (ii) different sets of experimental results existed ${ }^{642,675}$ (see also Fig. 1 of ref. 476). In regard to (i): the red squares in Fig. 49 result from QCT calculations assuming ${ }^{476}$ similar beam characteristics as found for other $\mathrm{H}_{2}$ beams used by the Rendulic group. Specifically, beam parameters were obtained ${ }^{43}$ by fitting TOF distributions from a thesis ${ }^{648}$ coming from the same group, as used successfully before to compare with experiments ${ }^{649}$ on $\mathrm{H}_{2}+\mathrm{Cu}(111)$ from the same group. ${ }^{43}$ Using beam parameters ${ }^{43,476}$ characterizing more narrow beams characteristic of experiments ${ }^{641}$ by the group of Rettner and Auerbach (green triangles in Fig. 49), it was possible to show that the comparison to experiment should not really be affected by the beam parameters used for the weakly activated $\mathrm{H}_{2}+\mathrm{Ni}(111)$ reaction for $E_{i}$ up to $0.25 \mathrm{eV} .{ }^{476}$ In regard to (ii): the Rendulic group published two sets of data for $\mathrm{H}_{2}+\mathrm{Ni}(111)$, which differed at high $\mathrm{E}_{i}$. In our comparison we have favored the later experiments ${ }^{675}$ (black crosses in Fig. 49) over the earlier ones ${ }^{642}$ (blue squares in Fig. 49), but the cause of the differences was not discussed by the experimentalists and remains unknown.

Three additional observations remain to be made regarding $\mathrm{H}_{2}+\mathrm{Ni}(111) .{ }^{476}$ First, the SRP-DF for $\mathrm{H}_{2}+\mathrm{Pt}(111)^{156}$ did not yield an accurate description of sticking of $\mathrm{H}_{2}$ on $\mathrm{Ni}(111)$, even though Ni and Pt belong to the same group. Second, as was the case for $\mathrm{H}_{2}+\mathrm{Ru}(0001),{ }^{151}$ the PBE-vdW2 is a c-SRP DF for $\mathrm{H}_{2}+$ $\mathrm{Ni}(111)$, while the other c-SRP DF for $\mathrm{H}_{2}+\mathrm{Ru}(0001)$ (PBE:RPBE(50:50)-vdW1) also gave a good description of $\mathrm{H}_{2}+$ $\mathrm{Ni}(111)$ (note that $\mathrm{Ru}$ is a group 8 and $\mathrm{Ni}$ a group 10 element). Third, as found for $\mathrm{H}_{2}+\mathrm{Ru}(0001),{ }^{151}$ these two DFs occupied close-lying positions in a plot of the energetic corrugation $v s$. minimum $E_{\mathrm{b}}$ (Fig. $4 \mathrm{a}$ in ref. 476) similar to Fig. 48.

5.2.3. $\mathbf{H}_{2}+\operatorname{Pt}(211)$. The SRP-DF for $\mathrm{H}_{2}+\operatorname{Pt}(111)^{156}$ also gives a chemically accurate description of DC of $\mathrm{H}_{2}$ and $\mathrm{D}_{2}$ on 


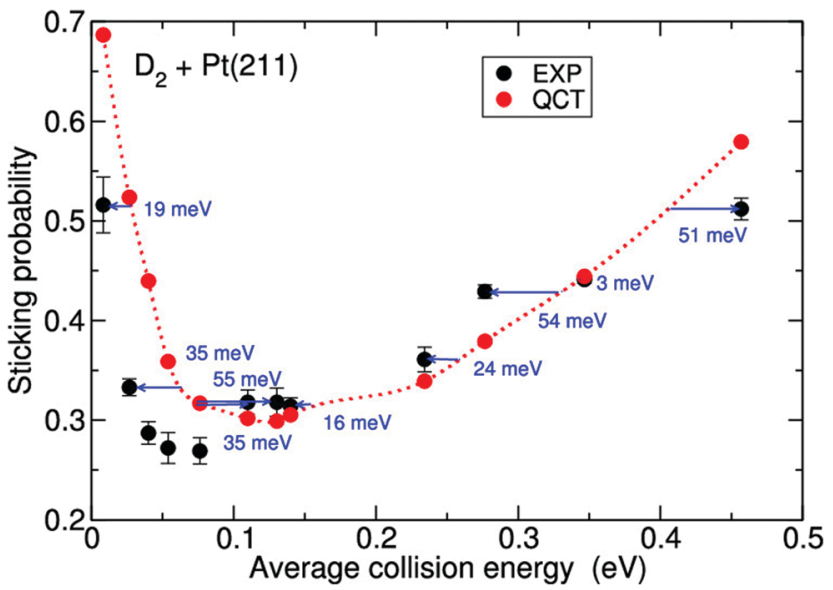

Fig. 50 The $S_{0}$ computed $^{48}$ for $D_{2}+\operatorname{Pt}(211)$ (red circles) with the QCT method using a PES calculated with the SRP DF derived ${ }^{156}$ for $\mathrm{H}_{2}+\mathrm{Pt}(111)$ are compared with experimental values ${ }^{732}$ (black symbols). The arrows and accompanying numbers show the collision energy difference between the interpolated theoretical and the experimental results. Reprinted from ref. 48 (https://pubs.acs.org/doi/10.1021/acs.jpcc.8b11018). Further permission requests to be directed to the ACS.

the stepped $\operatorname{Pt}(211)$ surface (Fig. 50 for $\mathrm{D}_{2}+\operatorname{Pt}(211)$ ), with the MD of the experimental data points to the spline interpolated theoretical curve being $0.75 \mathrm{kcal} \mathrm{mol}^{-1} \cdot{ }^{48}$ For $\mathrm{H}_{2}+\operatorname{Pt}(111)$ chemical accuracy was likewise achieved $\left(\mathrm{MD}=0.94 \mathrm{kcal} \mathrm{mol}^{-1}\right.$, see also Fig. 8 of ref. 48). As is the case for $\mathrm{H}_{2}+\mathrm{Ru}(0001)$, the sticking is not considerably affected by isotopic effects, and in the absence of a comparison to other experiments the $\operatorname{PBE} \alpha=$ 0.57-vdW2 DF is to be considered as a c-SRP DF for $\mathrm{H}_{2}+\mathrm{Pt}(211)$. Effects of ehp excitation on the sticking were investigated and found to be small. The difference seen between theory and experiment for low energies could be due to trapping-mediated reaction not being well described by classical mechanics. ${ }^{48}$ The differences at high $E_{i}$ could be due to the average $E_{i}$ being overestimated in the experiments: if these are taking according to $\left\langle E_{i}\right\rangle=2.7 k T_{\mathrm{n}}$, agreement with experiment is improved for $\mathrm{H}_{2}$ (see Fig. 11 of ref. 48).

5.2.4. $\quad \mathbf{N}_{2}+\mathbf{R u}(\mathbf{0 0 0 1})$. Although it has not been cast as a c-SRP DF yet, we argue that the RPBE ${ }^{196}$ DF is a c-SRP DF for $\mathrm{N}_{2}+\mathrm{Ru}(0001)$. The arguments for this may be found in Fig. 14 and 51, which show comparisons of QCT and MDEF calculations using an HDNN RPBE PES ${ }^{59}$ with experiments on $\mathrm{N}_{2}$ sticking ${ }^{210}$ on and scattering ${ }^{676}$ from $\mathrm{Ru}(0001)$. Fig. 14 shows that the computed $S_{0}$ fall between experimental errors bars as long as surface atom motion is modeled (BOMS, LDFA, and ODF), and regardless of whether or not (BOMS vs. LDFA/ODF), and if so how (LDFA or ODF) ehp excitation is modeled. ${ }^{59}$ Fig. 51A shows excellent agreement between QCT and experimental ${ }^{676}$ results for energy loss in translational motion to the surface, again as long as surface atom motion is modeled. ${ }^{59}$ Furthermore, Fig. 51B shows that the average energy transfer to vibration obtained with MDEF and using ODF is in agreement with the experimentally determined ${ }^{676}$ upper bound. ${ }^{59}$ The reasons that we only call the RPBE DF a c-SRP DF for $\mathrm{N}_{2}+\mathrm{Ru}(0001)$ are that (i) the error bars in the measured $S_{0}$ are currently too large to

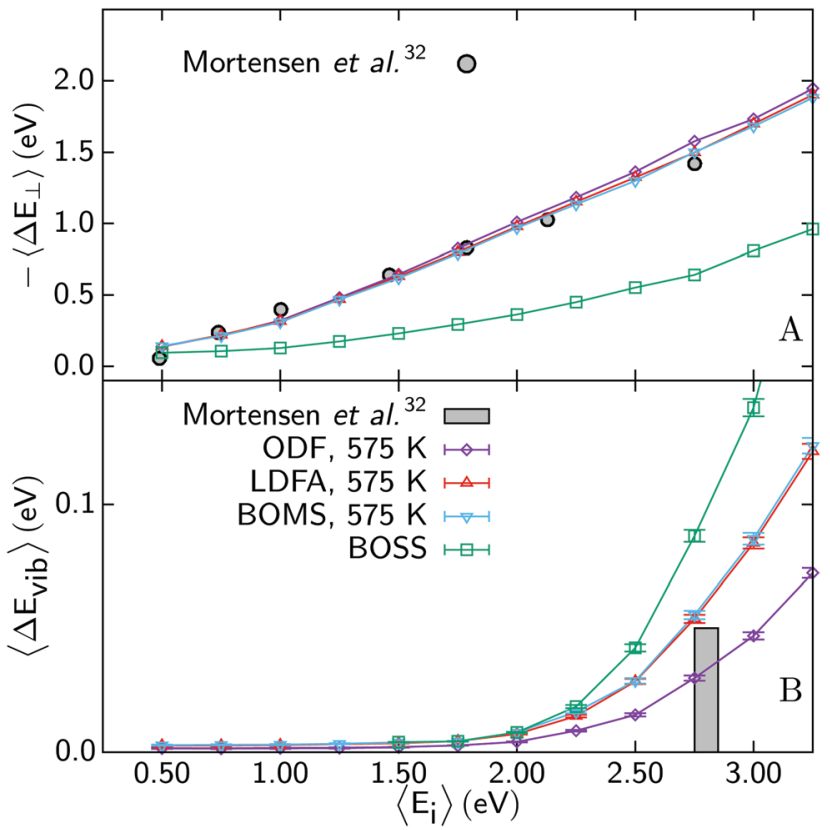

Fig. 51 (A) Average translational energy loss along the surface normal $-\left\langle\Delta E_{\perp}\right\rangle$ and $(B)$ average change of the vibrational energy $\left\langle\Delta E_{\text {vib }}\right\rangle$ as a function of the average incidence energy $\left\langle E_{i}\right\rangle$ for $N_{2}$ scattered from $\mathrm{Ru}(0001)$. Results from adiabatic calculations according to the BOSS model (green squares), the BOMS model (blue triangles),the moving surface LDFA model (red triangles), and the moving surface ODF model (purple diamonds) are plotted for $T_{\mathrm{s}}=$ 575 K. Experimental data ${ }^{676}$ (gray circles) are shown for comparison in (A). In (B), the maximum vibrational energy change of $0.05 \mathrm{eV}$ at $\left\langle E_{i}\right\rangle=2.8 \mathrm{eV}$ estimated in the same study ${ }^{676}$ is indicated (gray bar). Taken from ref. 59 (https://pubs.acs.org/doi/10.1021/acs.jpclett.9b00523). Further permission requests to be directed to the $\mathrm{ACS}$.

allow a verdict on the accuracy of the DF used for sticking (see Fig. 14), and (ii) it is not yet clear whether ODF is a better method than LDFA to model DC on metals, which casts some doubt on the accuracy of the RPBE-based results for vibrational excitation (Fig. 51B). Concerning sticking the ball now lies in the court of the experimentalists, who should be able to provide measurements of the accuracy required to put SRP-DFs on a firm basis. ${ }^{59}$ However, we note that $\mathrm{N}_{2}+\mathrm{Ru}(0001)$ has also been cast as a test case of whether it is best to use the LDFA or ODF to model DC on metals, which leads to additional challenges for theorists in determining an SRP-DF for this system (see also Sections 4.1.3 and 4.1.4, and ref. 59).

\subsection{Systems for which attempts to derive SRP-DFs have so far failed}

5.3.1. $\quad \mathbf{H}_{2}+\mathbf{A g}(111) . S_{0}$ computed ${ }^{152,186}$ with SRP-DFs for $\mathrm{H}_{2}+$ $\mathrm{Cu}(111)$ (the GGA SRP48, ${ }^{175}$ and the mGGA MS-PBEl ${ }^{152}$ and MSB86bl $^{152}$ DFs) for $\mathrm{D}_{2}+\mathrm{Ag}(111)$ have been compared with experimental values. ${ }^{677}$ In the work employing the SRP48 DF, results were computed according to narrow shifted Gaussian velocity distributions provided by the experimentalists, ${ }^{186,647}$ and according to narrow flux-weighted velocity distributions ${ }^{43}$ characterizing $\mathrm{D}_{2}+\mathrm{Cu}(111)$ experiments. ${ }^{12}$ The best comparison $\left(\mathrm{MD}=7.0 \mathrm{~kJ} \mathrm{~mol}^{-1}\right)$ was obtained using the latter distributions, which also have the more plausible form from a physical point 


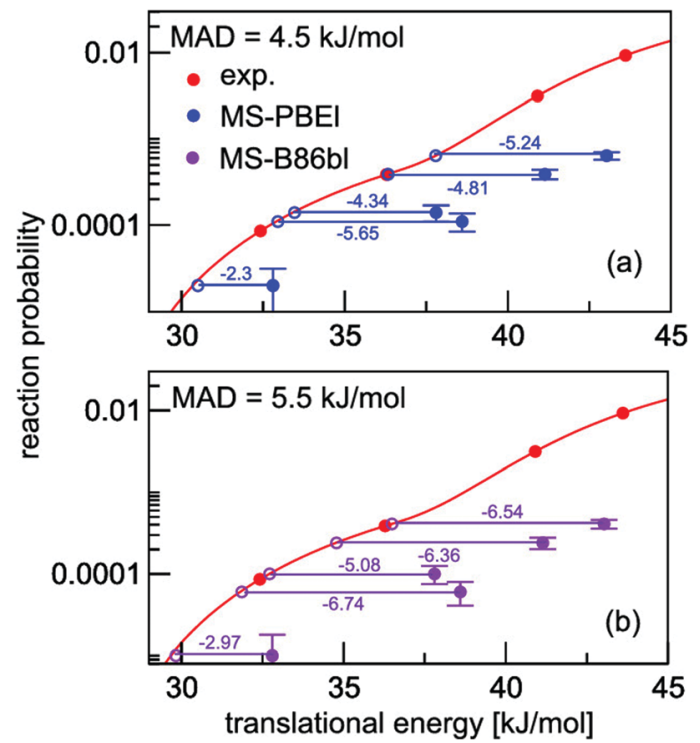

Fig. $52 S_{0}$ computed ${ }^{152}$ for $D_{2}+A g(111)$ with the MS-PBEl and MS-B86bl mGGA DFs are compared with measured values. ${ }^{677}$ Also indicated are the distances along the translational energy axis (the $x$-axis in the plot) between the computed and the interpolated experimental $S_{0}$ in $\mathrm{kJ} \mathrm{mol}^{-1}$. Reprinted from ref. 152 (https://pubs.acs.org/doi/10.1021/acs.jpca.9b02914). Further permission requests to be directed to the ACS.

of view (see Fig. 7 of ref. 186). Accordingly we have used the flux weighted $\mathrm{MB}$ parameters in the subsequent work using mGGA SRP-DFs, but note that the MB parameters remain an uncertainty in theoretical work on this system.

The use of mGGA SRP-DFs for $\mathrm{H}_{2}+\mathrm{Cu}(111)$ yielded a considerable improvement for $\mathrm{D}_{2}+\mathrm{Ag}(111)$, with the $\mathrm{MD}$ coming down from 7.0 to $4.5 \mathrm{~kJ} \mathrm{~mol}^{-1}$ with the MS-PBEl DF (Fig. 52a) and $5.5 \mathrm{~kJ} \mathrm{~mol}^{-1}$ with the MS-B86bl DF (Fig. 52b). ${ }^{152}$ The decrease was less than expected from the $E_{\mathrm{b}}$ only, which were lower by 3.6-10.2 $\mathrm{kJ} \mathrm{mol}^{-1}$ with MS-B86bl than with SRP48. This is most likely because the value of $r$ at the barrier $\left(r_{\mathrm{b}}\right)$ also shifted to lower values ${ }^{152}$ going from SRP48 to MS-B86bl. This probably lowers the contribution to the reaction from the initially highly excited vibrational states (which were $52 \%$ and $31 \%$ for $v=3$ and 4 , respectively, for the highest $E_{i}=0.486 \mathrm{eV}$ $\left(T_{\mathrm{n}}=2012 \mathrm{~K}\right)$ investigated using the SRP48 $\left.\mathrm{DF}^{186}\right)$. Calculations using DFs combining GGA exchange with vdW2 correlation, which are SRP DFs for $\mathrm{H}_{2}+\mathrm{Cu}(111)$, also performed better than the calculations with the SRP48 DF, with the PBE $\alpha 57-v d W 2 \mathrm{DF}$ reproducing the sticking experiments with almost chemical accuracy $\left(\mathrm{MD}=4.3 \mathrm{kcal} \mathrm{mol}^{-1}\right){ }^{658}$

The results suggest that SRP-DFs constructed on a higher rung of Perdew's ladder (mGGA instead of GGA, Fig. 4) or employing vdW correlation instead of GGA correlation may show better transferability than ordinary GGA DFs. Improved agreement with experiment can perhaps be obtained by using a SRP-DF with even better transferability (barriers lower than obtained with SRP48, but with positions not shifted to lower values of $r$ ). However, the dynamical method may also still be improved, e.g. by modeling surface atom motion (which on the logarithmic scale of Fig. 52 might have a big effect for the low $S_{0}$ measured), and by using a QD rather than the QCT method (QCT vs. QD being tested only down to $S_{0} \approx 10^{-3}$ for highly activated dissociation of $\mathrm{H}_{2},{ }^{511}$ see Fig. 16). New and betterdefined experiments would also be welcomed.

5.3.2. $\mathrm{H}_{2}+\operatorname{Pd}(111)$. For $\mathrm{H}_{2}+\operatorname{Pd}(111)$ there is also the problem that rather differing sets of measured $S_{0}$ exist $^{678-682}$ (see Fig. 1 of ref. 158, and also Fig. 53 for the most recent results from the Rendulic group ${ }^{680,681}$ and those of Gostein and Sitz ${ }^{682}$ ). A limited attempt was made to fit an SRP-DF for $\mathrm{H}_{2}+$ $\operatorname{Pd}(111)$ to the latter two experiments using QC and QCT calculations. $^{158}$ The DFs tested were the PBE, ${ }^{195}$ RPBE $^{196}$ PBE-vdW1, ${ }^{195,326}$ and PBE $\alpha$-vdW $1^{326,382}$ with $\alpha=0.5$ DFs. The strategy followed was to first test the comparison of QCT $S_{0}$ to the best experimental results obtained for $\mathrm{E}_{i}>125 \mathrm{meV},{ }^{680,681}$ for which the sticking should not be affected much by trapping, so that the QCT method should work well. ${ }^{63}$ This suggested the PBE-vdW1 DF as a good c-SRP DF (see Fig. 53). However, this DF did not yield $S_{0}$ comparing well with the best experiments for lower $E_{i}$ if QD was used to compute $S_{0}$ (see Fig. 53). ${ }^{158}$

Possibly the effort failed because the first fitting attempt compared to the newest experiments from the Rendulic group, which were performed at $T_{\mathrm{s}}=223 \mathrm{~K} .{ }^{681}$ This is well below the $\mathrm{H}_{2} / \mathrm{Pd}$ desorption temperature of $350 \mathrm{~K}^{682}$ It is therefore conceivable that the measured $S_{0}$ did not show a strong enough upturn at the higher $E_{i}$ (as hinted at by the $S_{0}$ measured by Gostein and Sitz at $T_{\mathrm{s}}=423 \mathrm{~K}$, see Fig. 53) because the surface was getting covered with $\mathrm{H}$ at the higher fluxes at higher $E_{i}$.

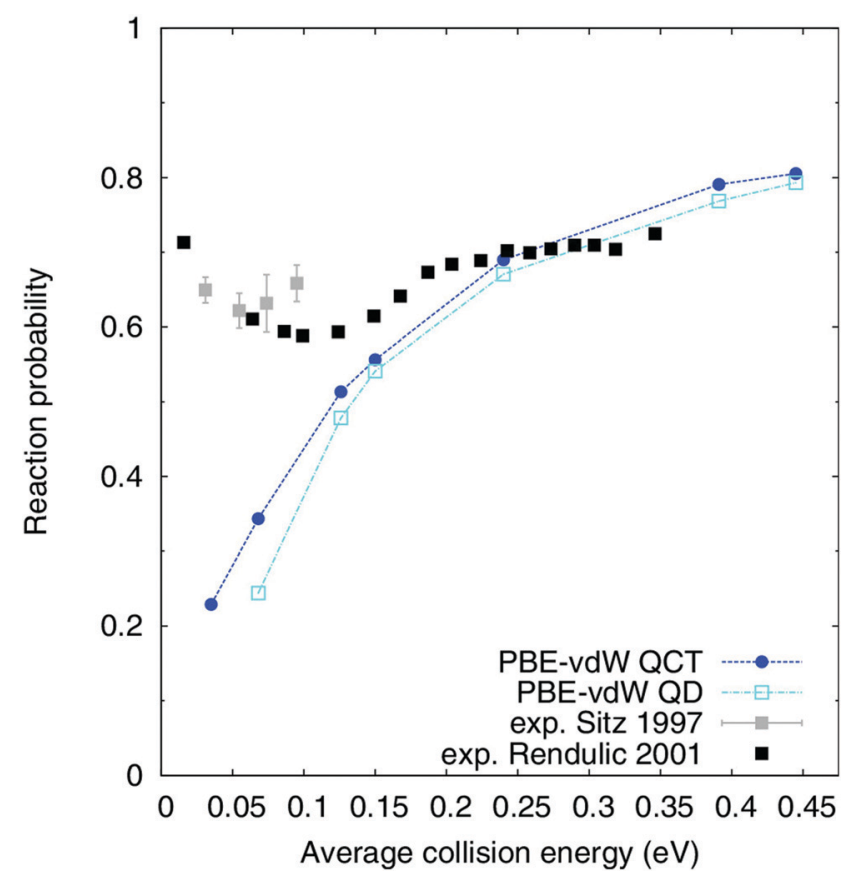

Fig. $53 \mathrm{~S}_{0}$ computed $^{158}$ for $\mathrm{H}_{2}+\mathrm{Pd}(111)$ with the PBE-vdW DF using the QCT method and QD are compared with values measured by Gostein and Sitz ${ }^{682}$ and by Rendulic and co-workers. ${ }^{680,681}$ All results are shown as a function of normal incidence energy. Reprinted from [J. M. Boereboom, M. Wijzenbroek, M. F. Somers and G. J. Kroes, Towards a specific reaction parameter density functional for reactive scattering of $\mathrm{H}_{2}$ from $\mathrm{Pd}(111)$, J. Chem. Phys., 2013, 139, 244707], with the permission of AIP Publishing. 
In future progress in determining the $S_{0}$ may be achieved by fitting an SRP-DF to the measurements reported by Gostein and Sitz $^{682}$ only, and validating the results through comparison with their $j$-resolved sticking and rotationally inelastic scattering probabilities. ${ }^{682}$ Uncertainties regarding the measured $S_{0}$ at higher $E_{i}$ than investigated by Gostein and Sitz ${ }^{682}$ might be removed by new experiments at a $T_{\mathrm{s}}$ exceeding $350 \mathrm{~K}$, which would help new theoretical efforts. While there may be some influence of $T_{\mathrm{s}}$ and surface atom motion on the measured and computed $S_{0},{ }^{475}$ this influence might be small enough not to hamper efforts to fit an SRP-DF with the BOSS model and using QD. Finally, it might also be possible to obtain an SRP-DF on the basis of QCT calculations using a Gaussian binning procedure and adiabaticity correction, ${ }^{596}$ as discussed in Section 4.2 (see also Fig. 21).

5.3.3. $\mathbf{H}_{2}+\mathbf{C O} / \mathbf{R u}(\mathbf{0 0 0 1})$. The transferability of one of the c-SRP DFs for $\mathrm{H}_{2}+\mathrm{Ru}(0001)$ (i.e., the PBE-vdW2 DF ${ }^{195,326}$ ) to $\mathrm{H}_{2}+$ CO-precovered $\mathrm{Ru}(0001)$ exhibiting a $(\sqrt{3} \times \sqrt{3}) R 30^{\circ}$ geometry has been investigated with DFMD calculations. ${ }^{188}$ This followed earlier work employing the BOSS model and using the RPBE DF. ${ }^{684}$ Making the static surface approximation was found not to have a large effect on the computed $S_{0}{ }^{188}$ The dynamics calculations based on the RPBE DF and the PBE-vdW2 DF both substantially underestimate ${ }^{188,684}$ the measured ${ }^{685}$ values (see Fig. 54). An uncertainty in the experiments concerns the coverage of the surface by $\mathrm{CO}$, which was not checked with LEED. ${ }^{188}$ It would be useful to future theoretical efforts to develop SRP-DFs if the experiments were repeated with such checks incorporated. If the surface coverage was as stated in the experiments the present comparison suggests that SRP-DFs developed for a specific molecule interacting with a specific metal surface may not be transferable to the same molecule interacting with the same metal surface pre-covered with adsorbed molecules. The calculations did show a dependence of the DFMD results on the size of the surface unit cell used (Fig. 54) $)^{188}(\mathrm{a}(3 \times 3)$ cell for the bare underlying $\mathrm{Ru}(0001)$ surface being necessary to ensure independent motion of the

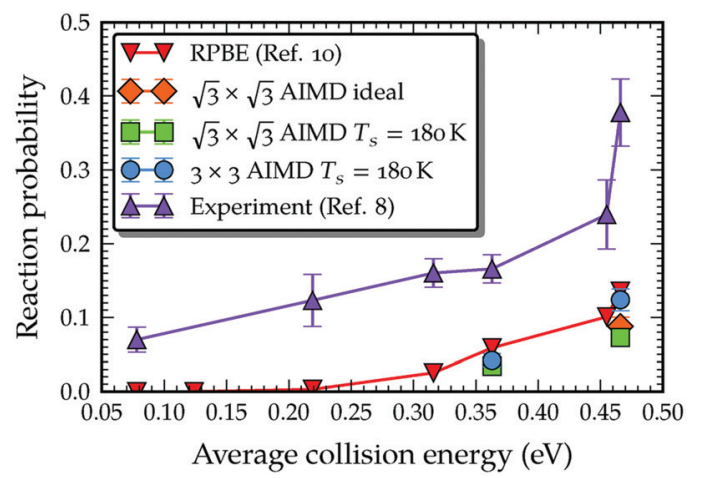

Fig. $54 S_{0}$ computed $^{188}$ using DFMD with the PBE-vdW2 DF for $D_{2}+$ $(\sqrt{3} \times \sqrt{3}) R 30^{\circ} \mathrm{CO} / \mathrm{Ru}(0001)$, using different sized surface unit cells and approximations to the surface atom motion are compared to measured values. $^{685}$ In the "AIMD ideal" calculations the CO-molecules and Ru surface atoms were kept fixed at their ideal geometries. Static surface results obtained ${ }^{684}$ using the RPBE DF are also shown. Reproduced from ref. 188 with permission from the PCCP Owner Societies.
CO molecules), but we expect the results using the $3 \times 3$ cell to be reasonably well converged.

5.3.4. $\quad \mathbf{N}_{2}+\mathbf{W}(\mathbf{1 1 0})$ and $\mathbf{W}(\mathbf{1 0 0})$. DFMD calculations ${ }^{88,101,149}$ have been used to model experiments ${ }^{211,212}$ on sticking of $\mathrm{N}_{2}$ to $\mathrm{W}(110)$ with several DFs. Here we focus on calculations ${ }^{88,149}$ that modeled the effect of $T_{\mathrm{s}}$ and surface atom motion, as these yield results that differ considerably from calculations employing the BOSS approximation. ${ }^{88} \mathrm{~N}_{2}$ on $\mathrm{W}(110)$ is yet another example of a system for which differing sets of measured $S_{0}$ are available for normal incidence ${ }^{211,212}$ (Fig. 55, top panel). Of the DFs tested (PBE, ${ }^{195} \mathrm{RPBE}^{196}$ and the original vdW2 $\left.{ }^{327} \mathrm{DF}\right)$ the vdW2 DF gives the best overall agreement with experiment, although it underestimates the measured $S_{0}$ for off-normal incidence at $E_{i}=2.29 \mathrm{eV}$. The later experiments performed at normal incidence ${ }^{212}$ gave $S_{0}$ smaller than the ones originally measured $^{211}$ by a factor $1.4 .^{149}$ If the experiments performed later are indeed the most accurate ones, and if the correction factor applicable to off-normal incidence is the same, then the agreement of the vdW2 DFMD results with experiment should be further improved. New experiments aimed at testing this assumption would be useful.

Martin-Gondre et al. ${ }^{101}$ also performed DFMD calculations on $\mathrm{N}_{2}$ scattering from $\mathrm{W}(110)$ with the optPBE-vdW1 DF. ${ }^{331}$

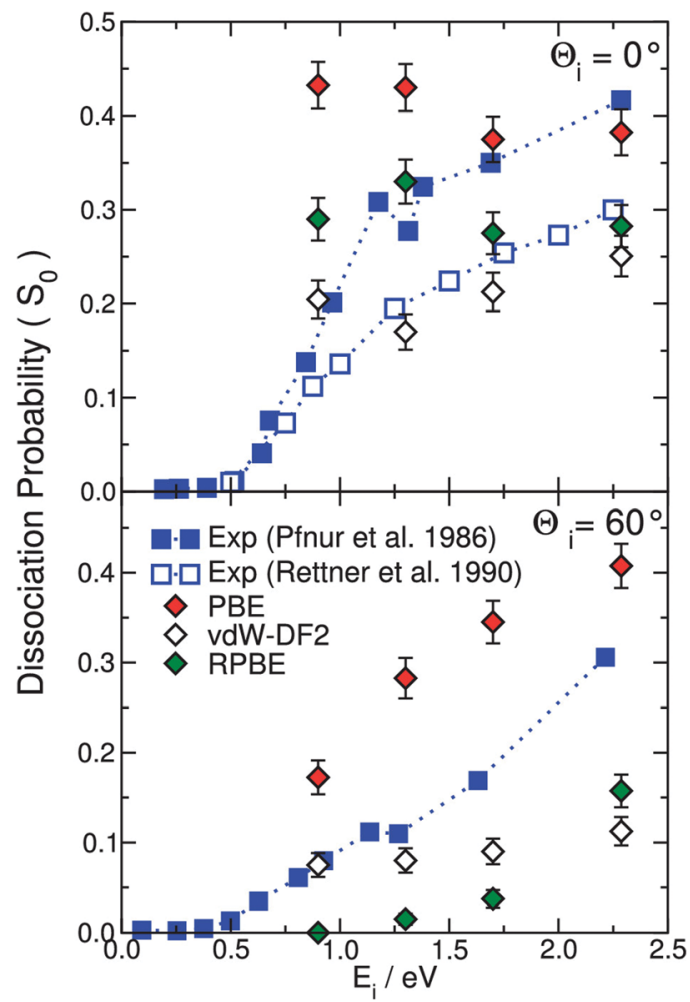

Fig. $55 S_{0}$ computed $^{149}$ with the vdW2 DF and computed ${ }^{88}$ with the PBE and RPBE DFs for $\mathrm{N}_{2}+\mathrm{W}(110)$ at normal incidence (upper panel) and off-normal incidence (lower panel) are shown as a function of incidence energy, and compared with measured ${ }^{211,212}$ values. Reprinted from [D. Migliorini, F. Nattino and G. J. Kroes, Application of van der Waals functionals to the calculation of dissociative adsorption of $\mathrm{N}_{2}$ on $W(110)$ for static and dynamic systems, J. Chem. Phys., 2016, 144, 084702], with the permission of AIP Publishing. 
Their $S_{0}$ compared rather well with experiment, but their results may be less reliable as they used the static surface approximation. ${ }^{101}$ Computed final rotational state distributions in scattering ${ }^{110}$ were compared with experimental results based on classical dynamics calculations using a PW91 and a RPBE PES, also using the static surface approximation. In many, but not all cases good quantitative agreement was achieved ${ }^{110}$ with experiments. ${ }^{686}$

Crespos and co-workers ${ }^{132}$ carried out QCT calculations on sticking of $\mathrm{N}_{2}$ to $\mathrm{W}(100)$, using the GLO approach to incorporate surface atom motion and $T_{\mathrm{S}}$, and testing the PW91 ${ }^{312}$ and vdW $2^{327}$ DFs. While the measured $S_{0}$ were still overestimated substantially, far better agreement with experiment ${ }^{687}$ was achieved employing the vdW2 than the PW91 DF (see Fig. 56). ${ }^{132}$ Assuming a large degree of transferability between $\mathrm{N}_{2}+\mathrm{W}(110)$ and $\mathrm{W}(100)$, and recognizing the similarity ${ }^{195}$ between PW91 and PBE, these results give support to the hypothesis discussed above that the $S_{0}$ measured for $\mathrm{N}_{2}+\mathrm{W}(110)$ at normal incidence by Rettner et al. ${ }^{212}$ were more accurate than those of Pfnür et al. ${ }^{211}$ (the PBE (PW91) data overestimating the experimental data of Rettner et al. in Fig. $55^{212}$ (Fig. $\left.56^{687}\right)$ ). Additionally, the results for $\mathrm{N}_{2}+\mathrm{W}(100)^{132}$ then suggest that the $S_{0}$ measured for $\mathrm{N}_{2}+\mathrm{W}(110)$ at off-normal incidence should be decreased, further improving agreement with the vdW2 results for this system (Fig. 55). Crespos and co-workers ${ }^{143}$ also investigated the additional effect of ehp excitation modeled with the LDFA approach, finding no considerable influence on sticking on $\mathrm{W}(100)$. However, they did find that molecular chemisorption increased at the cost of DC at low $E_{i}{ }^{143}$ With the vdW2 DF Crespos and co-workers ${ }^{146}$ also found better agreement with experiments on angular distributions ${ }^{212}$ and trapping-desorption fractions in scattering as a function of $T_{\mathrm{s}}^{688}$ than obtained with the PW91 DF.

Fitting an SRP-DF to sticking data for $\mathrm{N}_{2}+\mathrm{W}(110)$ and $\mathrm{W}(100)$ is complicated as the system combines dissociative with molecular chemisorption in deep molecular chemisorption wells. The SRP-DF therefore has to be good at describing direct

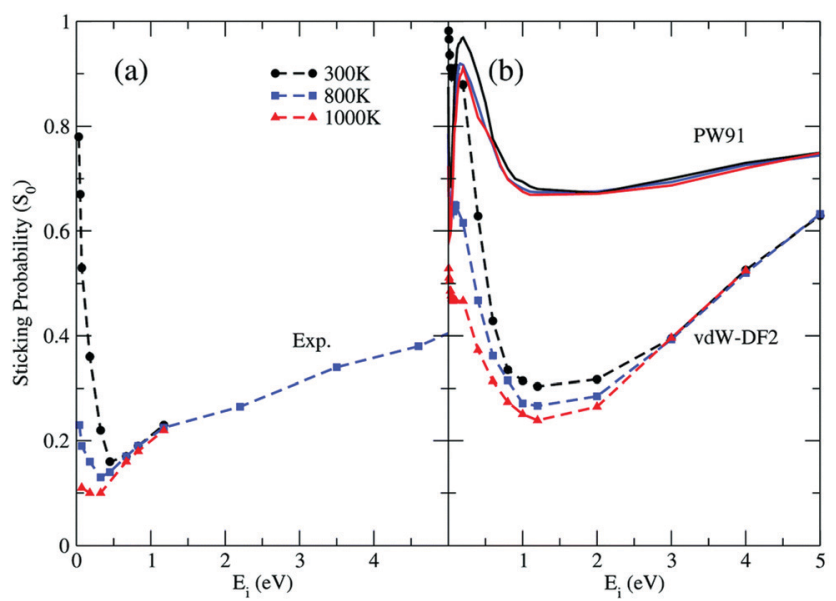

Fig. $56 S_{0}$ measured ${ }^{208,607}$ for $N_{2}+W(100)^{132}$ for three different values of $T_{\mathrm{s}}$ (a) and computed ${ }^{132}$ with QCT calculations using the GLO model for surface atom motion and using the PW91 and vdW2 DFs (b) are compared. The results are shown as a function of incidence energy. Reprinted from ref. 132 with permission from the PCCP Owner Societies.
DC over barriers, molecular chemisorption, and the transfer from molecular chemisorption to DC wells over barriers. Here, a problem may be that DFs good at describing molecular chemisorption may perform less well at describing activated dissociation, just like the best DF describing gas phase thermochemistry is not necessarily the best DF describing gas phase $E_{\mathrm{b}}{ }^{689}$

5.3.5. $\quad \mathrm{O}_{2}+\mathrm{Al}(\mathbf{1 1 1})$. The $\mathrm{O}_{2}+\mathrm{Al}(111)$ system is of intrinsic interest as a benchmark system for which GGA DFs have notoriously failed to reproduce measured $S_{0} \cdot{ }^{570}$ Specifically, purely CT calculations (no initial zpe) using the BOSS model and PESs computed with the $\mathrm{PBE}^{195}$ and $\mathrm{RPBE}^{196} \mathrm{DFs}^{570}$ both overestimate measured $S_{0}{ }^{217}$ for normal incidence, treating the reaction as non-activated instead of activated (see Fig. 7, where the RPBE data can also be viewed as representing PBE data). This means trial SRP-DFs based on eqn (2a) or (2b) would fail if they were to be based on the PBE and RPBE GGAs, and few GGA DFs are more repulsive than RPBE (BLYP ${ }^{313,353}$ and RPBELYP ${ }^{196,353}$ being examples (Fig. 48), but note that the LYP correlation DF does not satisfy the constraint of an appropriate description of the homogeneous electron gas, ${ }^{690}$ and therefore poorly describes metals, ${ }^{691}$ see also Fig. 5). As already mentioned in Section 2.4 much better results can be obtained using a high level $a b$ initio method with DFT embedding ${ }^{35}$ (see Fig. 7).

Very recently QCT calculations have been performed ${ }^{351}$ on $\mathrm{O}_{2}+\mathrm{Al}(111)$ that used the mGGA MS RPBEl DF ${ }^{152}$ discussed earlier and the screened hybrid HSE03-1/3X DF. The HSE03-1/ $3 \mathrm{X}$ DF is based on the HSE03 $\mathrm{DF}^{692,693}$ with the maximum fraction of exact exchange increased from $1 / 4$ to $1 / 3$. The use of both DFs leads to activated dissociation with the HSE03-1/3X DF producing the best results, although not yet as good as the results obtained with the ECW PES (see Fig. 7 and Fig. 2 of ref. 351). The improved agreement obtained with the screened DF relative to the RPBE GGA DF could to some extent have been anticipated on the basis of calculations with hybrid functions showing barriers for $\mathrm{O}_{2}$ dissociation on an $\mathrm{Al}_{22}$ cluster ${ }^{694}$ and on an $\mathrm{Al}(111)$ slab. $^{350}$ It is likely that further improved agreement with experiment can be obtained by combining a screened exchange functional with a higher maximum fraction of exact exchange with vdW1 or vdW2 correlation. ${ }^{351}$

5.3.6. $\mathbf{H C l}+\mathbf{A u ( 1 1 1 )}$. Sticking of DCl to $\mathrm{Au}(111)$ has been investigated with DFMD using the RPBE $\mathrm{DF}^{136}$ and with QD using the PW91 DF. ${ }^{105}$ Sticking of $\mathrm{HCl}$ to $\mathrm{Au}(111)$ has been investigated with DFMD using the PBE and the RPBE DF, ${ }^{85}$ with DFMD and DFMDEF using the SRP32-vdW1 and revPBE-vdW1 DFs, ${ }^{147}$ with MD and MDEF using the RPBE DF and a HDNNP, ${ }^{133}$ with MD using the MS-RPBEl DF and a HDNNP, ${ }^{695}$ and with QD using both the PW91 and RPBE DFs. ${ }^{106,134}$ All these calculations have compared with experimental results for sticking. ${ }^{219,695}$

A typical example of a comparison of computed ${ }^{133,134,147,695}$ and measured $^{219,695} S_{0}$ is shown in Fig. 57. QCT calculations ${ }^{133}$ that used the RPBE DF and modeled surface atom motion (orange diamonds) overestimated the previously published experimental $^{219} S_{0}$ (green open squares) by more than 1 and up to 3 orders of magnitude, depending on $E_{i}$. This was also true for DFMD calculations ${ }^{85}$ (see e.g. Fig. 3 of ref. 134) and QD calculations ${ }^{134}$ using the RPBE DF (orange dashed line in Fig. 57b). 


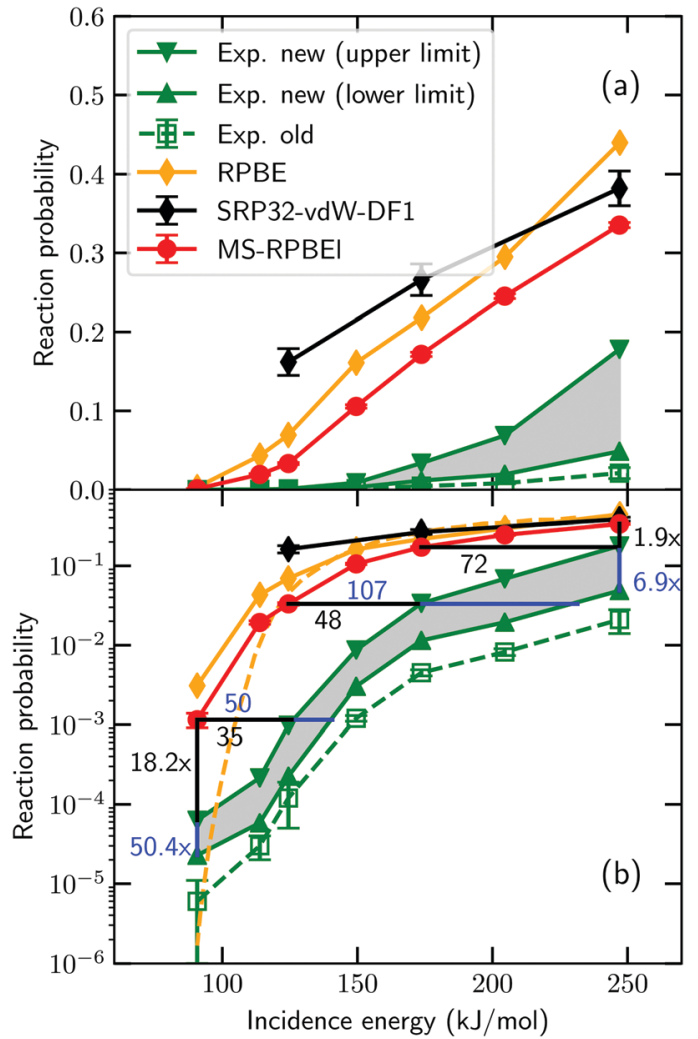

Fig. 57 Sticking probabilities computed for $\mathrm{HCl}+\mathrm{Au}(111)$ with the MS-RPBEI DF, ${ }^{695}$ the RPBE DF, ${ }^{133}$ and the SRP32-vdW1 DF ${ }^{147}$ are compared to experimental results, for normal incidence. The open green squares are the experimental sticking probabilities first published, ${ }^{219}$ while the upper base and lower base triangles represent upper and lower bounds to the experimental $S_{0}$ obtained from an improved analysis of the experiments. ${ }^{695}$ Panel a uses a linear and panel $b$ a logarithmic scale for $S_{0}$. In panel b results of $\mathrm{QCT} T^{133}$ (orange diamonds connected by solid organge line) and of $\mathrm{QD}^{134}$ calculations using the RPBE DF are presented. Reprinted from ref. 695 (https://pubs.acs.org/doi/10.1021/acs.jpcc.0c03756). Further permission requests to be directed to the ACS.

An earlier analysis of the experiments as first published suggested that the measured $S_{0}$ could well be underestimated by a factor $2-3 .^{85} \mathrm{~A}$ thorough reanalysis of the experiments indeed led to increased lower and upper bounds to the experimental $S_{0}{ }^{695}$ (compare the gray shaded areas with the open green squares in Fig. 57). However, the comparison clearly shows that the dynamics calculations based on the repulsive RPBE DF still overestimate the measured reactivity substantially. DFMD and DFMDEF calculations using the SRP32-vdW1 and revPBE-vdW1 DFs gave somewhat lower computed $S_{0}$ (see Fig. 2 and Table 5 of ref. 147), which however still overestimate the experimental $S_{0}$ shown in Fig. 57 substantially. Also, scaled transition probabilities computed with the SRP32-vdW1 DF for vibrational excitation from $v=1$ to $v=2$ overestimated experimental results by factors $3-8 .^{147}$ These ${ }^{147}$ and other ${ }^{133}$ calculations also suggest that the choice of the DF affects the computed $S_{0}$ much more than approximations made concerning whether surface atom motion and/or ehp excitation (within the LDFA) are allowed. The calculations also suggested that the solution does not lie with combining a GGA exchange and a vdW correlation $\mathrm{DF}^{147}$ (see also Fig. 57).
Like the work on $\mathrm{O}_{2}+\mathrm{Al}(111)$ the research on $\mathrm{HCl}+\mathrm{Au}(111)$ emphatically raises the question of which type of SRP-DF can be used to accurately describe the experiments. $\mathrm{HCl}+\mathrm{Au}(111)$ is thus another example of a system posing considerable challenges to theory. ${ }^{85,219}$ Fig. 57 shows that using a MS type $^{695}$ mGGA functional (i.e., the MS-RPBEl $\mathrm{DF}^{152}$ ) leads to a somewhat improved agreement with experiment, as it did for the $\mathrm{O}_{2}+\mathrm{Al}(111)$ system. ${ }^{351}$ It is likely that further improved results can be obtained with a screened hybrid functional as found for $\mathrm{O}_{2}+\mathrm{Al}(111),{ }^{351}$ but computing a PES with a screened hybrid DF may well be much more expensive for $\mathrm{HCl}+\mathrm{Au}(111)$ than for $\mathrm{O}_{2}+\mathrm{Al}(111)$ due to the higher number of valence electrons of $\mathrm{Au}$.

5.3.7. $\mathrm{D}_{2} \mathrm{O}+\mathrm{Ni}(\mathbf{1 1 1})$. Jiang and Guo and coworkers ${ }^{120,155}$ have performed approximate 9D QD calculations on the sticking of $\mathrm{D}_{2} \mathrm{O}$ to $\mathrm{Ni}(111)$ using a PW91 ${ }^{312}$ PES and a RPBE ${ }^{196}$ PES. Their calculations used the SAED approximation: full 7D QD results were obtained for a sufficient number of fixed impacts site of $\mathrm{D}_{2} \mathrm{O}$, and the 7D $S_{0}$ were then averaged (see Section 4 ). The LRS method was used to take into account $T_{\mathrm{s}}$. Their PW91 and RPBE $S_{0}$ are compared with measured ${ }^{78} S_{0}$ in Fig. 24 .

Although agreement to chemical accuracy was not yet achieved, the RPBE results are actually quite reasonable (semi-quantitative agreement), especially for the $1 \nu_{3}$ state. However, the RPBE results overestimate the measured values for the $2 \nu_{3}$ state. The PW91 results clearly overestimate the measured $S_{0}$ for both these initial states. Note that the comparison between experimental laser-off and computed $v=0$ results is not meaningful ${ }^{155}$ in view of the high $T_{\mathrm{N}}$ used in the experiments $(573-773 \mathrm{~K}) .^{78}$

It will be a considerable challenge to develop a SRP-DF for $\mathrm{H}_{2} \mathrm{O}+\mathrm{Ni}(111)$. Possible improvements ${ }^{155}$ include SRP-DFs based on GGA exchange and NL van der Waals correlation, modeling the initial rotational state (which in the experiments on vibrationally excited $\mathrm{D}_{2} \mathrm{O}$ was not equal to the $J=0$ state modeled), and investigating the validity of the LRS method used to describe the effect of $T_{\mathrm{S}}$. The validity of the SAED model (needed in QD) has only been demonstrated by comparison to full 9D calculations on $\mathrm{H}_{2} \mathrm{O}+\mathrm{Cu}(111),{ }^{61}$ where it was not demonstrated on the logarithmic scale ${ }^{61}$ of Fig. 24, but on a linear scale (Fig. 23). At the same time, the applicability of the DFMD and QCT methods has been put in doubt by the QD-QCT comparison made recently (see also Fig. 20). ${ }^{597}$ In summary $\mathrm{D}_{2} \mathrm{O}+\mathrm{Ni}(111)$ is another example of a system posing considerable challenges to the development of an SRP-DF.

5.3.8. $\quad \mathbf{N H}_{3}+\mathbf{R u}(\mathbf{0 0 0 1})$. The PBE $\mathrm{DF}^{696}$ has been tested in QCT calculations on $\mathrm{NH}_{3}+\mathrm{Ru}(0001)$ using the BOSS approximation. ${ }^{66}$ The computed $S_{0}$ are compared with experimental values ${ }^{245}$ in Fig. 58. The computed $S_{0}$ considerably overestimate the measured values.

The RPBE-vdW1 $\mathrm{DF}^{196,326}$ has been tested in QCT calculations on the same system, using a HDNNP to describe the effect of the surface mobility. ${ }^{535}$ With the RPBE-vdW1 DF, a higher $E_{\mathrm{b}}\left(63.2 \mathrm{~kJ} \mathrm{~mol}^{-1}\right)$ was obtained $^{535}$ than with the PBE DF $\left(45.6 \mathrm{~kJ} \mathrm{~mol}^{-1}\right)$. Therefore lower $S_{0}$ values were obtained with the RPBE-vdW1 DF, ${ }^{535}$ although the computed $S_{0}$ still overestimate the measured values ${ }^{245}$ at the value of $T_{\mathrm{s}}$ at which the sticking was not affected by trapping in the experiments (1100 K, Fig. 58). 


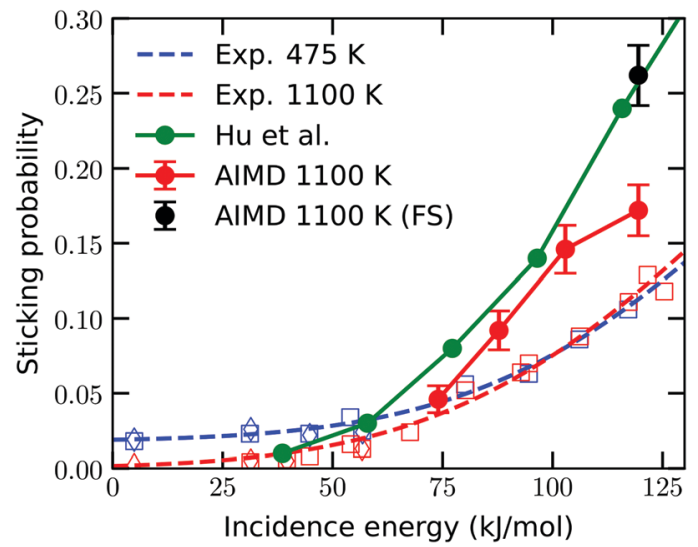

Fig. $58 \mathrm{~S}_{0}$ for $\mathrm{NH}_{3}+\mathrm{Ru}(0001)$. Computed $\mathrm{S}_{0}$ are indicated by closed circles and measured ${ }^{245} S_{0}$ by open diamonds and squares, of which the diamonds and squares are measurements using hydrogen or nitrogen desorption, respectively. Results computed ${ }^{535}$ with DFMD and the RPBEvdW1 DF are shown for a mobile (red circles) and a fixed (black circles) surface, and previous results computed with the QCT method while keeping the surface fixed (closed green circles) are from ref. 66. Results obtained for $T_{\mathrm{s}}=475$ and $1100 \mathrm{~K}$ are represented by blue and red symbols, respectively. Reprinted from ref. 535 (https://pubs.acs.org/doi/10.1021/ acs.jpcc.9b09121). Further permission requests to be directed to the ACS.

It is unclear whether a DF consisting of GGA exchange and $\mathrm{vdW}^{326}$ or vdW2 $2^{327}$ correlation, a pure GGA, or a mGGA DF can be found which will enable reproducing the measurements. The RPBEvdW1 DF is already quite repulsive, although possibly higher barriers can be obtained with the RPBE ${ }^{196}$ or RPBE-vdW2 ${ }^{196,327}$ DFs.

5.3.9. $\mathrm{CHD}_{3}+\mathbf{P t}(\mathbf{2 1 0})$ and $\operatorname{Pt}(\mathbf{1 1 0})-(2 \times 1)$. The SPR32-vdW1 DF, which is an SRP-DF for $\mathrm{CHD}_{3}+\operatorname{Pt}(111)$ and $\mathrm{Pt}(211),{ }^{47}$ has also been tested in DFMD calculations comparing with $\mathrm{MB}$ sticking experiments on $\mathrm{CHD}_{3}+\operatorname{Pt}(110)-(2 \times 1) \cdot{ }^{182}$ This missing row reconstructed $\mathrm{Pt}(110)$ surface may be considered as another example of a stepped surface, like $\operatorname{Pt}(211)$, but of a different kind, with the "terrace" being as broad as the "step", but facing in the opposite direction. As Fig. 59A shows, surprisingly poor agreement with experiment was obtained: ${ }^{182}$ The calculations severely underestimated the computed $S_{0}\left(\mathrm{MD}=4.8 \mathrm{kcal} \mathrm{mol}^{-1}\right)$. A problem with the DFMD was that considerable trapping occurred, with energy conversion from translational motion normal to motion parallel to the surface. If one assumes that all molecules still trapped after 1 ps simulation time go on to react agreement with experiment improves, but the MD still is equal to $2.5 \mathrm{kcal} \mathrm{mol}^{-1}$ (Fig. 59B). ${ }^{182}$ An investigation of the geometry of the Pt(110)$(2 \times 1)$ surface geometry computed with the SRP32-vdW1 DF suggests this to be another potential source of error: For two of the three experimental surface geometries investigated, the computed $E_{\mathrm{b}}$ goes down by 1.5 and $2.3 \mathrm{kcal} \mathrm{mol}^{-1}$, respectively, relative to the $E_{\mathrm{b}}$ for the SRP32-vdW1 geometry. ${ }^{182}$ Probably the main culprit is the vertical distance between the exposed valley atom and the atom below the ridge atom, which is most likely overestimated with the SRP32-vdW1 DF. ${ }^{182}$

The SPR32-vdW1 DF has also been tested in DFMD calculations comparing with $\mathrm{MB}$ sticking experiments on $\mathrm{CHD}_{3}+\operatorname{Pt}(210) .{ }^{181}$ $\mathrm{Pt}(210)$ is a kinked surface. Again no agreement to within chemical
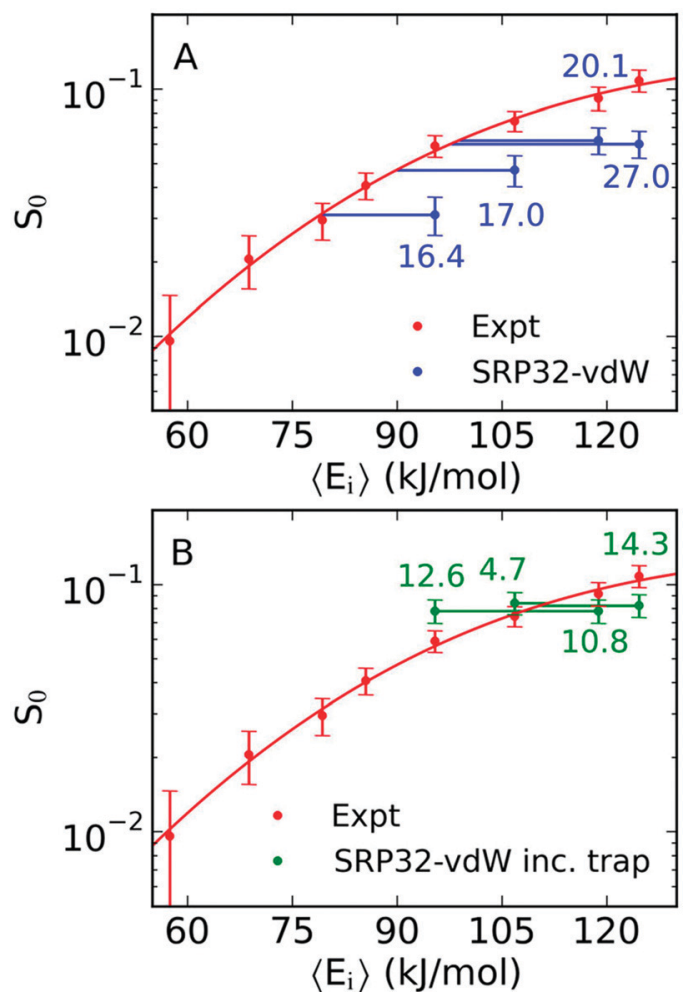

Fig. 59 Comparison of measured $S_{0}$ with values computed with DFMD calculations using the SRP32-vdW2 DF for $\mathrm{CHD}_{3}$ dissociation on $\mathrm{Pt}(110)$ $(2 \times 1)$ at a surface temperature of $650 \mathrm{~K}(\mathrm{a})$. The red line shows an S-shape curve fit to the experimental data, and the numbers represent the energy shift in $\mathrm{kJ} \mathrm{mol}^{-1}$ between the calculated sticking coefficients and the fit. In panel $b$ trajectories classified as trapping have also been counted as reacted in obtaining the computed $S_{0}$. Reprinted from $[\mathrm{H}$. Chadwick, A. Gutiérrez-González, R. D. Beck and G. J. Kroes, Transferability of the SRP32-vdW specific reaction parameter functional to $\mathrm{CHD}_{3}$ dissociation on Pt(110)-(2 × 1), J. Chem. Phys., 2019, 150, 124702], with the permission of AIP Publishing.

accuracy was obtained (Fig. 60): ${ }^{181}$ The calculations overestimated the measured $S_{0}\left(\mathrm{MD}=3.25 \mathrm{kcal} \mathrm{mol}^{-1}\right.$, not considering cases where computed $S_{0}$ had to be compared with extrapolated experimental values). ${ }^{181}$ Trapping was not a major problem in the calculations, and assuming trapped molecules go on to react would now lead to an even larger MD. Using a surface geometry closer to the experimental one is unlikely to result in better agreement with experiment. ${ }^{181}$ A possible ${ }^{181}$ cause of the disagreement between theory and experiment is that roughening of the $\operatorname{Pt}(210)$ surface occurred in the experiment.

\subsection{Calculations using SRP-DFs for related systems}

5.4.1. $\quad \mathbf{H}_{2}+\mathbf{A u}(111)$. Predictions of $R_{v j}\left(E_{i}\right)$ were made ${ }^{187}$ for $\mathrm{H}_{2}+\mathrm{Au}(111)$ using the QCT method based on PESs computed with six different DFs, including the SRP48 and the optPBE-vdW1 DFs which may be viewed as SRP-DFs for $\mathrm{H}_{2}+\mathrm{Cu}(111) .{ }^{150,175}$ The calculations predicted highly activated DC, with a minimum $E_{\mathrm{b}}$ of $1.32 \mathrm{eV}$ for the SRP48 DF. Also, high $\eta$ were obtained, i.e., $\eta_{v=0 \rightarrow 1}=0.81$ (0.83) for $J=3 \mathrm{H}_{2}\left(J=2 \mathrm{D}_{2}\right) .{ }^{187}$ The SRP48 predictions were later tested in associative desorption experiments. ${ }^{23}$ The experiments showed very good agreement with the calculations 


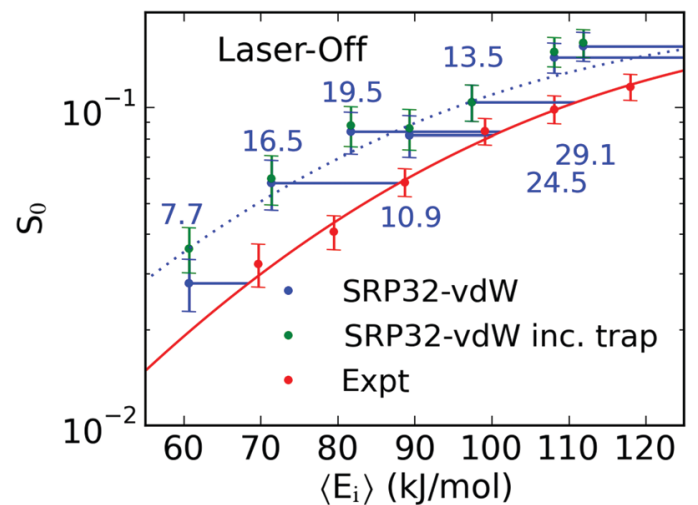

Fig. 60 Comparison of the $\mathrm{S}_{0}$ for $\mathrm{CHD}_{3}+\mathrm{Pt}(210)$ measured (red circles) and calculated with DFMD using the SRP32-vdW2 DF, including (green circles) and excluding (blue circles) the contribution from trapped trajectories, under laser-off conditions. The red line shows an S-shaped curve fit to the experimental data, the dotted blue line shows the fit shifted by $13.8 \mathrm{~kJ} \mathrm{~mol}^{-1}$, and the blue numbers indicate the shift (in $\mathrm{kJ} \mathrm{mol}^{-1}$ ) between the interpolated experimental and the calculated sticking coefficients. The results are shown as a function of incidence energy. Reprinted with permission from ( $\mathrm{H}$. Chadwick, A. Gutiérrez-González, R. D. Beck and G. J. Kroes, $\mathrm{CHD}_{3}$ dissociation on the kinked Pt(210) surface: A comparison of experiment and theory, J. Phys. Chem. C, 2019, 123, 14530-14539). Copyright (2019) American Chemical Society.

for the $\eta$ and for vibrational state populations for $\mathrm{D}_{2}+\mathrm{Au}(111)$ (e.g. $v=1 / v=0$ population ratios of $0.33 \pm 0.01$ and 0.36 were obtained in the experiments and in the SRP48 theory). ${ }^{23}$ However, theory was found to under-predict the $v=1 / v=0$ population ratio for $\mathrm{H}_{2}+\mathrm{Au}(111)(0.51 \pm 0.01$ vs. 0.26 in the experiments and the SRP48 theory, respectively, see also Fig. 61). Also, compared to the experiments the computed ADEs were too high by $0.23-0.29 \mathrm{eV}$ for $(v=0,1) \mathrm{H}_{2}$ and $\mathrm{D}_{2}$ desorbing from $\mathrm{Au}(111) .{ }^{23}$ Part of this difference could come from the calculations having been done with the BOSS model, neglecting the high experimental $T_{\mathrm{s}}(1061 \mathrm{~K})$. The experimental $R_{v j}\left(E_{i}\right) v s$. $E_{i}$ curves should therefore be broader, leading to lower ADEs (see also Fig. 33B for $\mathrm{H}_{2}+\mathrm{Cu}(111)$ and its discussion in Section 5.1.1). The experimentalists attributed the discrepancies in the computed and measured ADEs and $v=1 / v=0$ population ratio for $\mathrm{H}_{2}$ to the absence of an ehp excitation channel in the calculations. $^{23}$

The $\mathrm{H}_{2}+\mathrm{Au}(111)$ system was later revisited with theory ${ }^{658}$ using the mGGA MS-PBEl and the optPBE-DF1, PBE $\alpha 57-v d W 2$, B86SRP68-vdW2 DFs discussed in Section 5.1.1. With all these DFs the computed $E_{0}^{v j}$ parameters were too high by $90-110 \mathrm{meV}$; again, the best results were obtained with the PBE DF. ${ }^{658}$ The $v=1 / v=0$ ratio in desorption was severely underestimated, ${ }^{658}$ just like it was with the SRP48 DF. Taking into account the surface reconstruction, the high value of $T_{\mathrm{s}}$ in the experiments $(1063 \mathrm{~K})$, and, possibly, non-adiabatic effects might lead to better agreement with experiment in future. ${ }^{658}$ The presence of experimental sticking probabilities from well-defined molecular beam experiments on this system might also help. ${ }^{658}$

5.4.2. $\mathrm{H}_{2}+\mathbf{C u}(211)$. The SRP48 DF developed for $\mathrm{H}_{2}+$ $\mathrm{Cu}(111)^{175}$ has also been used in QCT calculations on $\mathrm{D}_{2}+\mathrm{Cu}(211)$

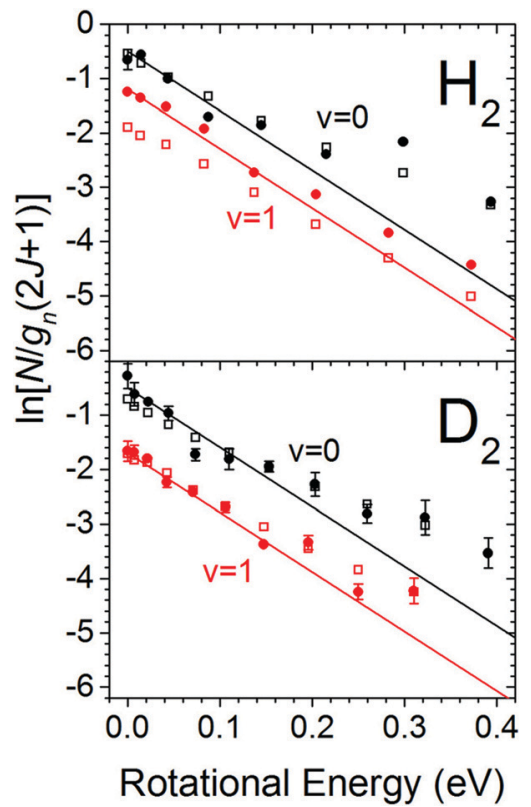

Fig. 61 Measured ${ }^{23}$ state distributions (filled circles) for $\mathrm{H}_{2}$ and $\mathrm{D}_{2}$ desorbing from $\mathrm{Au}(111)$ are compared with computed ${ }^{23}$ state distributions (open squares) based on QCT calculations employing the SRP48 DF. ${ }^{187}$ The slopes of the lines are representative of rotational Boltzmann distributions at the experimental $T_{\mathrm{s}}(1061 \mathrm{~K})$. Reprinted with permission from (Q. Shuai, S. Kaufmann, D. J. Auerbach, D. Schwarzer and A. M. Wodtke, Evidence for electronhole pair excitation in the associative desorption of $\mathrm{H}_{2}$ and $\mathrm{D}_{2}$ from $\mathrm{Au}(111)$, J. Phys. Chem. Lett., 2017, 8, 1657-1663). Copyright (2017) American Chemical Society.

and $\mathrm{D}_{2}+\mathrm{Cu}(111)$ comparing to new $\mathrm{MB}$ sticking experiments on these systems. ${ }^{166}$ A surprising result was that the stepped $\mathrm{Cu}(211)$ system is less reactive towards $\mathrm{D}_{2}$ than the flat $\mathrm{Cu}(111)$ surface (Fig. 62a). ${ }^{166}$ Application of the d-band model ${ }^{697,698}$ suggests a lower $E_{\mathrm{b}}$ for $\mathrm{Cu}(211)$ as the center of its d-band is shifted towards the Fermi-level compared to $\mathrm{Cu}(111)$ (see Fig. 62b). ${ }^{166}$ Although this prediction turns out to be correct for top-to-bridge dissociation on $\mathrm{Cu}(111)$ and the (111) terrace of $\mathrm{Cu}(211)$, reaction is still favored on $\mathrm{Cu}(111)$ through a geometrical effect: the minimum $E_{\mathrm{b}}$ occurs over a bridge site on $\mathrm{Cu}(111)$, i.e., not over a top site, which becomes under-coordinated at the (100) step edge of $\mathrm{Cu}(211) .{ }^{166}$

New associative desorption experiments on $\mathrm{H}_{2}, \mathrm{HD}$, and $\mathrm{D}_{2}$ desorbing from $\mathrm{Cu}(111)$ and $\mathrm{Cu}(211)$ are in agreement with the reactivity on $\mathrm{Cu}(211)$ being lower than on $\mathrm{Cu}(111)$. These experiments have also revealed a "slow" associative desorption channel on both facets that is yet to be accounted for by theory. ${ }^{194}$ New QD and QCT calculations also using the SRP48 DF have provided $E_{1 / 2}^{v j}$ values ${ }^{511}$ in good agreement with the new experiments ${ }^{194}$ for $\mathrm{H}_{2}$ and $\mathrm{D}_{2}+\mathrm{Cu}(211)$; here, the $E_{1 / 2}^{v j}$ parameter stands for the $E_{i}$ at which $R_{v j}$ is half the value of the reaction probability at the maximum value of $E_{i}$ for which the reaction probability can still be reliably extracted from the associative desorption experiments using detailed balance. ${ }^{194}$ Chemical accuracy was achieved for $\mathrm{H}_{2}+\mathrm{Cu}(211)$ with QD and QCT (the MUEs of the computed $E_{1 / 2}^{v j}$ were $0.83 \mathrm{kcal} \mathrm{mol}^{-1}$ ). ${ }^{511}$ Going from QCT to MDEF using the LDFA in direct calculations on DC, agreement to within chemical accuracy was no longer 

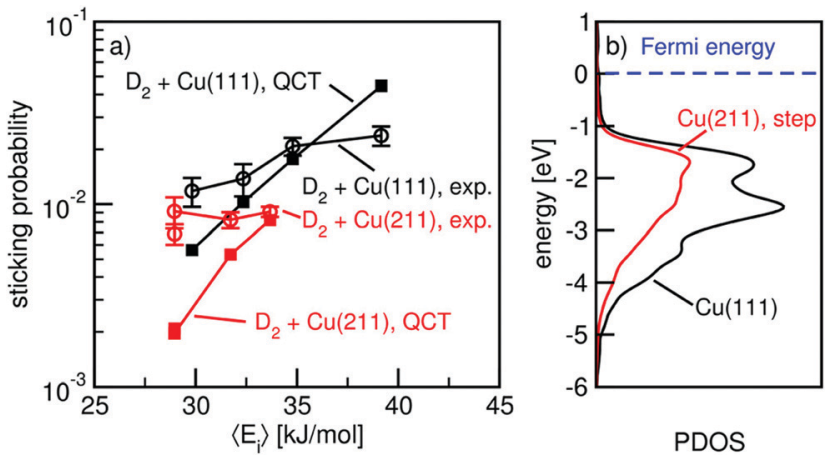

Fig. $62 S_{0}$ measured $^{166}$ for $D_{2}+C u(111)$ and $\mathrm{Cu}(211)$ are compared with values computed ${ }^{166}$ using the SRP48 DF (a). The results are shown as a function of incidence energy. Also shown are the site projected density of states (PDOS) of the $d$-band for first layer $\mathrm{Cu}(111)$ surface atoms and the $\mathrm{Cu}(211)$ step atoms (b). Reprinted from ref. 166 (https://pubs.acs.org/doi/ 10.1021/acs.jpclett.7b03097). Further permission requests to be directed to the ACS.

achieved (MUE $=1.17 \mathrm{kcal} \mathrm{mol}^{-1}$ ). Reasoning that applying EF in calculations directly modeling associative desorption would decrease the $E_{\mathrm{t}}$ of desorbed dihydrogen, $\mathrm{MDEF}^{*}$ values of $E_{1 / 2}^{v j}$ were obtained by subtracting the difference between the QCT and the MDEF values from the QCT values. This led to better agreement with experiment $\left(\mathrm{MUE}=0.55 \mathrm{kcal} \mathrm{mol}^{-1}\right) \cdot{ }^{511}$ The SRP48 DF is probably also an SRP DF for $\mathrm{H}_{2}+\mathrm{Cu}(211)$, but confirmation requires a comparison to molecular beam experiments over a larger range of incidence energies than used in ref. 166. Finally, we note that the optPBE-vdW1 DF, which is an SRP-DF for $\mathrm{H}_{2}+\mathrm{Cu}(111), \mathrm{Cu}(100)$, and $\mathrm{Cu}(110),{ }^{467}$ also gave an accurate description of associative desorption of $\mathrm{H}_{2}$ from $\mathrm{Cu}(211),{ }^{467}$ but as is the case for the SRP48 DF testing on additional experiments is required to call this DF an SRP-DF for $\mathrm{H}_{2}+\mathrm{Cu}(211)$.

5.4.3. HOD + Ni(111). $S_{0}$ computed for HOD + Ni(111) with DFMD simulations ${ }^{184}$ using the SRP32-vdW1 DF developed for $\mathrm{CHD}_{3}$ interacting with $\mathrm{Ni}(111)^{44}$ are compared with $S_{0}$ measured for $\mathrm{D}_{2} \mathrm{O}+\mathrm{Ni}(111)^{78}$ for lower $E_{i}$ in Fig. 63. It is hard to draw firm conclusions from this comparison. The $S_{0}$ for HOD should be higher than for $\mathrm{D}_{2} \mathrm{O}$ due to the higher vibrational frequencies of $\mathrm{HOD}$, in particular of the $\mathrm{OH}$ stretch. The calculations were meant to be predictive, and future experiments will hopefully show whether the calculations predicted too high $S_{0}$, as Fig. $63^{184}$ might seem to suggest. If the predicted $S_{0}$ would turn out to be too high this could be due to the SRP-DF not being transferable from $\mathrm{CH}_{4}+\mathrm{Ni}(111)$ to $\mathrm{H}_{2} \mathrm{O}+\mathrm{Ni}(111)$, but it could also be due to problems with zpe leakage (see Fig. 20 and its discussion in Section 4.2).

The DFMD calculations also revealed interesting mechanistic details. They suggested that the RAA used in the earliest RPH calculations should work well for water $\mathrm{DC}$ on $\mathrm{Ni}(111)$ : the initial orientational distribution of the dissociating $\mathrm{OH}$-bond appears random (like the sine distribution in Fig. 45C), while at the time of reaction the distribution is Gaussian-like and centered on an angle close to the TS value (like the distribution of the reacting molecules shown in Fig. 45C, see Fig. 7 of ref. 184).

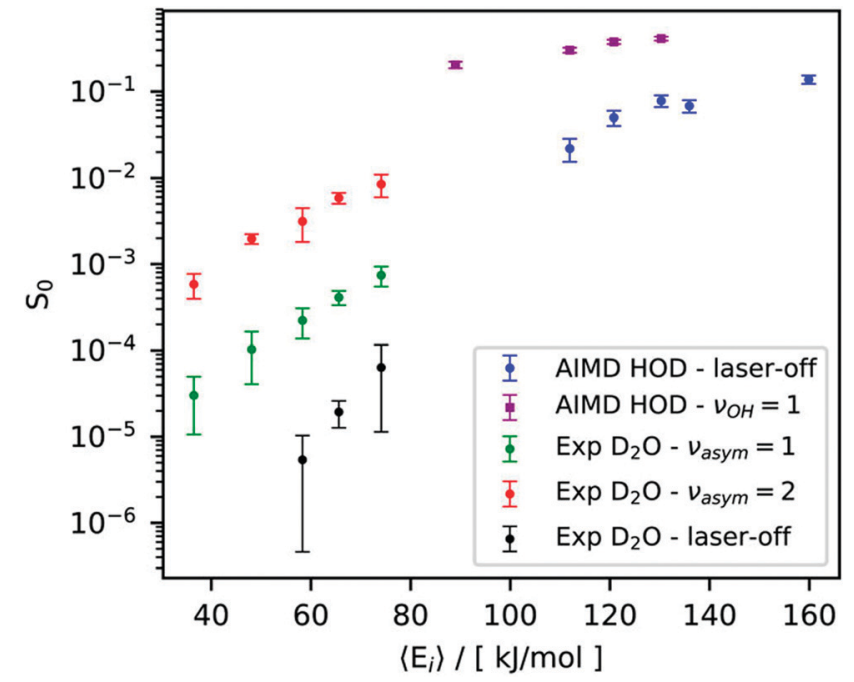

Fig. $63 S_{0}$ computed $^{184}$ with DFMD using the SRP32-vdW1 DF for dissociative chemisorption of HOD on Ni(111) for laser-off conditions and for the $\mathrm{OH}$-stretch vibration excited with one quantum are compared with experimental values for $\mathrm{D}_{2} \mathrm{O}+\mathrm{Ni}(111),{ }^{78}$ for laser-off conditions and for $\mathrm{D}_{2} \mathrm{O}$ excited with one quantum and two quanta of the asymmetric stretch vibration. All results are shown as a function of incidence energy, and are for normal incidence. Reprinted from [D. Migliorini, F. Nattino, A. K. Tiwari and G. J. Kroes, HOD on Ni(111): Ab Initio Molecular Dynamics Prediction of Molecular Beam Experiments, J. Chem. Phys., 2018, 149, 244706], with the permission of AIP Publishing.

Starting from the gas phase, the molecules reacting under laser-off

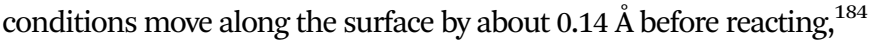
suggesting that the SAEX approximation tested for $\mathrm{H}_{2} \mathrm{O}+\mathrm{Cu}(111)^{61}$ (see Fig. 23 and its discussion in Section 4.4.2) might not work as well for HOD $+\mathrm{Ni}(111)$. This deserves further testing.

5.4.4. $\quad \mathbf{C H D}_{3}+\operatorname{Pd}(\mathbf{1 1 1}) \cdot S_{0}$ computed with the SRP32-vdW1 DF for $\mathrm{CHD}_{3}+\operatorname{Pd}(111),{ }^{533} \mathrm{Pt}(111),{ }^{47}$ and $\mathrm{Ni}(111)^{44}$ are compared in Fig. 64. The calculations for $\mathrm{CHD}_{3}+\operatorname{Pd}(111)^{533}$ are also meant as predictions; their test by experiments could answer the question of whether the SRP-DF for $\mathrm{CHD}_{3}+\mathrm{Pt}(111)$ and $\mathrm{Ni}(111)$ (i.e., the SRP32-vdW1 DF) is also transferable to $\mathrm{CHD}_{3}+$ $\operatorname{Pd}(111)$. The calculations predict that the reactivity of $\mathrm{CHD}_{3}+$ $\operatorname{Pd}(111)$ should be intermediate between the other two systems, $\mathrm{CHD}_{3}+\mathrm{Pt}(111)$ and $\mathrm{Ni}(111){ }^{533}$ This is also expected on the basis of the computed SRP32-vdW1 minimum $E_{\mathrm{b}}$ values, which are 97.9, 84.1, and $78.7 \mathrm{~kJ} \mathrm{~mol}^{-1}$ for $\mathrm{CHD}_{3}+\mathrm{Ni}(111), \operatorname{Pd}(111)$, and $\mathrm{Pt}(111)$, respectively. ${ }^{533}$ The calculations also predict that at high $E_{i}$ the laser-off reactivity on Pd becomes similar to that on Ni and much lower than that on Pt (Fig. 64) while the barriers are much closer on Pd and Pt. This has been explained ${ }^{533}$ on the basis of how the reactivity depends on the surface impact site, and reactivity at high $E_{i}$ being considerably diminished by the bobsled effect on both Pd and $\mathrm{Ni}$ (see also below for $\mathrm{CHD}_{3}+\mathrm{Cu}(111)$ ). It is possible to compare the $S_{0}$ for $\mathrm{CHD}_{3}+\mathrm{Pd}(111)$ predicted for $\mathrm{H}_{2}$ seeded beams ${ }^{533}$ to earlier experiments on $\mathrm{CH}_{4}+\operatorname{Pd}(111)$ using He seeded beams. ${ }^{699}$ Unfortunately, like the above predictions for HOD + Ni(111), this is difficult because the experiments and the theory were not only done for different methane isotopologues, but also for different $E_{i}$ ranges, and $T_{\mathrm{N}}{ }^{533}$ 


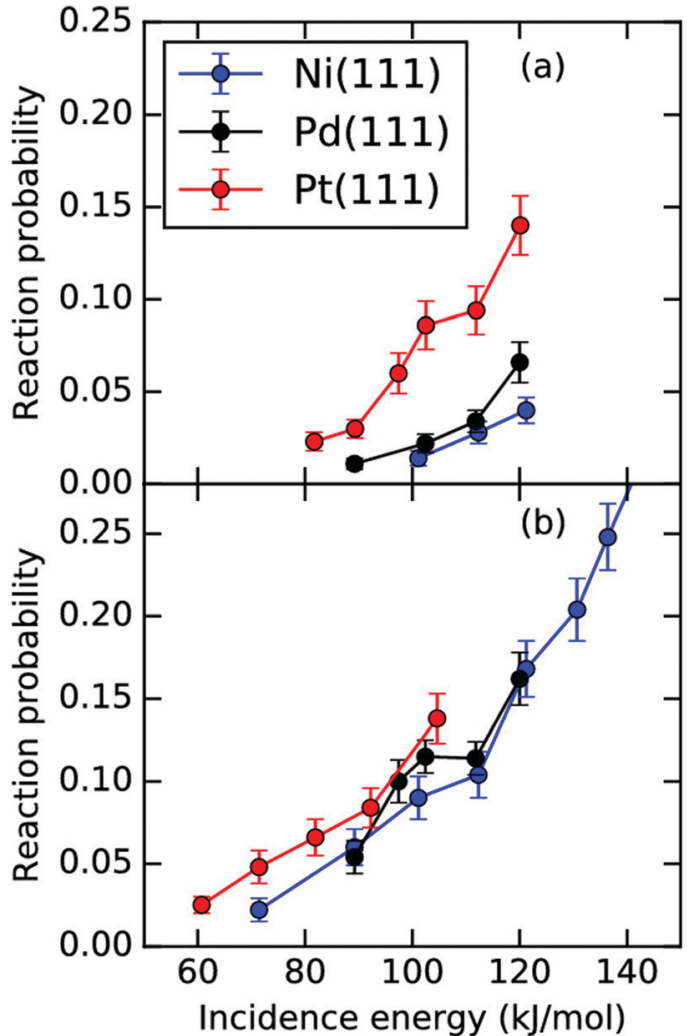

Fig. 64 So computed with DFMD using the SRP32-vdW1 DF for dissociative chemisorption of $\mathrm{CHD}_{3}$ on $\mathrm{Pd}(111),{ }^{533} \mathrm{Ni}(111),{ }^{44}$ and $\mathrm{Pt}(111)^{47}$ for laser-off conditions and for the $\mathrm{CH}$-stretch vibration excited with one quantum are compared with one another, for laser-off conditions (a), and for $\mathrm{CHD}_{3}$ excited with one quantum of $\mathrm{CH}$ stretch vibration (b). All results are shown as a function of incidence energy, and are for normal incidence. Reprinted from ref. 533 (https://pubs.acs.org/doi/10.1021/acs.jpcc.9b05757). Further permission requests to be directed to the ACS.

5.4.5. $\mathrm{CHD}_{3}+\mathrm{Cu}(111)$ and single atom surface alloys of Cu(111). Predictive DFMD ${ }^{185}$ and QCT calculations ${ }^{63}$ with the SRP32-vdW1 DF developed for $\mathrm{CHD}_{3}+\mathrm{Ni}(111)^{44}$ have also been done on the $\mathrm{DC}$ of $\mathrm{CHD}_{3}$ on $\mathrm{Cu}(111)$. The QCT calculations used a HD-NNP PES. ${ }^{63}$ Results are shown as a function of $E_{i}$ in Fig. 9a and as a function of total energy of $\mathrm{CHD}_{3}$ in Fig. 9b, for the initial vibrational ground state, $\nu_{1}=1$ and 2 , and for a $\mathrm{MB}$ simulation ("laser-off"). ${ }^{63} \mathrm{MB}$ experiments on $\mathrm{CHD}_{3}+\mathrm{Cu}(111)$ using $\mathrm{H}_{2}$ seeded beams would test the transferability of the SRP32-vdW1 DF from $\mathrm{CH}_{4}+\mathrm{Ni}(111)$ to $\mathrm{CH}_{4}+\mathrm{Cu}(111)$.

Fig. 9a shows that the QCT calculations using the HDNNP $\mathrm{PES}^{63}$ reproduced earlier DFMD simulations, ${ }^{185}$ proving the accuracy of the PES, which also describes the dependence of the moleculesurface interaction on surface atom displacements ${ }^{63}$ (see also Section 3.1). Fig. 9b shows that adding extra vibrational energy to go from initial $\nu_{1}=1$ to $\nu_{1}=2$ promotes the reaction more than adding the same amount of energy to incident translational motion. In other words, $\eta_{1 \rightarrow 2}$ (1.7) exceeds 1 , whereas $\eta_{0 \rightarrow 1}(0.8)$ is smaller than 1. Analysis of the trajectories showed that, at $E_{i}$ where the $S_{0}$ are similar, on the way to the barrier $\nu_{1}=1$ and $\nu_{1}=0 \mathrm{CHD}_{3}$ slide off the reaction path and on to the repulsive wall of the PES (Fig. 65a and b). This effect is called the
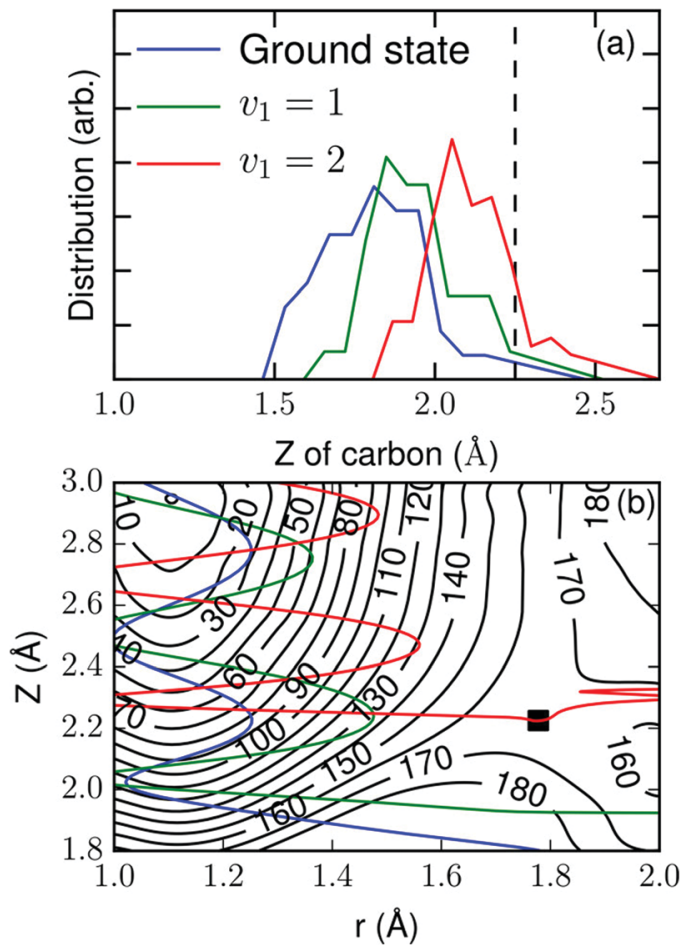

Fig. 65 Distributions are shown for the distance of the carbon atom to the surface $\left(Z_{C}\right)$ at the moment of dissociation, for $\mathrm{CHD}_{3}$ dissociating on $\mathrm{Cu}(111)$ and approaching the surface in its vibrational ground state, or the state in which the $\mathrm{CH}$-stretch mode is excited with one or two quanta (a). The vertical dashed line indicates the distance of the carbon-atom to the surface at the transition state. An elbow plot of the PES (contour line labels in $\mathrm{kJ} \mathrm{mol}^{-1}$ ) of $\mathrm{CHD}_{3}$ interacting with $\mathrm{Cu}(111)(\mathrm{b})$. In the plot, the PES is shown as it is minimized with respect to all DOFs except the distance of the molecule to the surface $(Z)$ and the dissociating $\mathrm{CH}$-bond distance $(\boldsymbol{r})$. Representative trajectories for which $\mathrm{CHD}_{3}$ goes on to react are shown for $\mathrm{CHD}_{3}$ in its initial vibrational ground state (blue), and in the states where the $\mathrm{CH}$-stretch mode is excited with one (green) or two (red) quanta, for collision energies for which the reaction probability is approximately $3 \times$ $10^{-4}$. Reprinted from ref. 63 (https://pubs.acs.org/doi/10.1021/acs.jpclett. 9 b00560). Further permission requests to be directed to the ACS.

bobsled effect. ${ }^{700-702}$ The reason that reaction of $\nu_{1}=2 \mathrm{CHD}_{3}$ is so efficient relative to $\nu_{1}=1$ is that the reactivity of the $\nu_{1}=1$ state is low due to the bobsled effect; it points to a low reactivity of the $\nu_{1}=1$ state rather than a high reactivity of $\nu_{1}=2 .^{63}$

DFMD calculations have also been performed on the DC of $\mathrm{CHD}_{3}$ on single atom surface alloys of $\mathrm{Cu}(111) .{ }^{185}$ It was found that the presence of $\mathrm{Pt}$ in $\mathrm{Cu}(111)$ enhanced the reactivity more than the presence of Pd (see Table 2 of ref. 185). This trend correlates well with how the presence of the group 10 metal atom in the surface lowered the minimum $E_{\mathrm{b}}$ (from $166.6 \mathrm{~kJ} \mathrm{~mol}^{-1}$ on $\mathrm{Cu}(111)$ to $142.5 \mathrm{~kJ} \mathrm{~mol}^{-1}$ on $\mathrm{Pd}-\mathrm{Cu}(111)$ and $134.1 \mathrm{~kJ} \mathrm{~mol}^{-1}$ on $\mathrm{Pt}-\mathrm{Cu}(111))$, and with the trend in reactivity of $\mathrm{CHD}_{3}$ on (111) surfaces of group 10 metals (Fig. 64). The reactivity differences among $\mathrm{Cu}(111), \mathrm{Pd} / \mathrm{Cu}(111)$ and $\mathrm{Pt} / \mathrm{Cu}(111)$ were also found to correlate with changes in the dynamical pathway and in the energy transfer from $\mathrm{CHD}_{3}$ to the surface. ${ }^{185}$

5.4.6. $\mathbf{C H}_{4}+\mathbf{N i ( 2 1 1 )}$. The SRP32-vdW1 DF developed for methane $+\mathrm{Ni}(111)^{44}$ has also been used in RPH calculations on 
$\mathrm{CH}_{4}+\mathrm{Ni}(211) .{ }^{179}$ The computed reaction probabilities are taken as sums of three contributions, i.e., from sticking near two step sites (the so-called $\mathrm{P}$ and $\mathrm{Q}$ paths) and from sticking near a terrace site (the T path). ${ }^{179} \mathrm{MB}$ experiments, which are not yet available for $\mathrm{CH}_{4}+\mathrm{Ni}(211)$, and which would measure initial state-selected and laser-off $S_{0}$ for comparison to the predicted $S_{0}$ curves $^{179}$ would constitute tests of the transferability of the SRP-DF for $\mathrm{CH}_{4}+\mathrm{Ni}(111)$ to $\mathrm{CH}_{4}+\mathrm{Ni}(211)$.

Jackson and coworkers were also able to compute thermal $S_{0}$ for sticking to the step site and the terrace site ${ }^{178,179}$ and to compare these to experimental results obtained for these sites at $500 \mathrm{~K}$ from measurements on $\mathrm{CH}_{4}+\mathrm{Ni}(141313),{ }^{703}$ and to additional measurements for the (111) surface. ${ }^{704,705}$ Like the (211) surface, the (14 13 13) surface consists of (100) steps and (111) terraces, with the latter however being much wider 27 rows on average $\mathrm{e}^{703}$ ) than on the (211) surface. Results were obtained by thermally averaging $R_{v j}\left(E_{i}\right)$ computed with the RPH method (open squares in Fig. 66). ${ }^{178,179}$ Results were also obtained with two versions of transition state theory (TST) (which should be the more appropriate method for computing thermal $S_{0}{ }^{178}$ ). A harmonic version of TST was used (dashed lines in Fig. 66), and a more sophisticated version with anharmonic couplings between the lowest frequency modes (solid lines in Fig. 66). The coupled TST values ${ }^{178}$ are in excellent agreement with the experimental results extracted from measurements for the $\left(\begin{array}{lll}14 & 13 & 13\end{array}\right)$ surface, for both the step and the terrace. ${ }^{703}$ The $S_{0}(T)$ measured on the (111) surfaces $^{704,705}$ overestimate the coupled TST and the

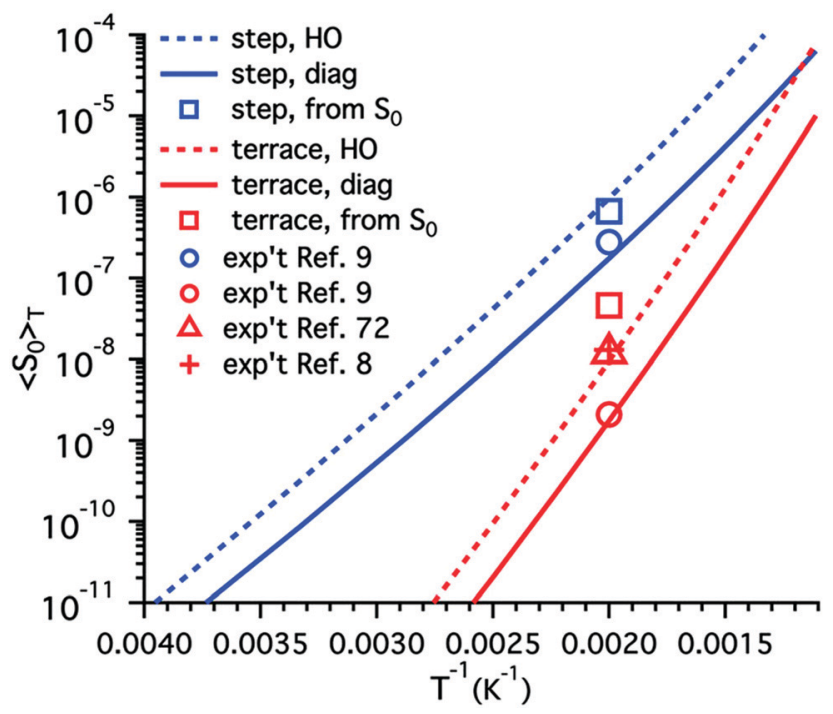

Fig. 66 The average thermal sticking probability is shown for $\mathrm{CH}_{4}$ dissociating on a step site (blue curves and points) or terrace site (red curves and points) of $\mathrm{Ni}(211)$, as a function of inverse temperature. ${ }^{178}$ The computational results (lines) were computed with transition state theory, using either the harmonic approximation (dashed lines) for the soft modes, or a "diagonalization treatment" (full lines). Experimental results are shown with points. For the details and the references to the experiments, see ref. 178. Reprinted from [H. Guo and B. Jackson, Methane dissociation on stepped Ni surfaces resolved by impact site, collision energy, vibrational state, and lattice distortion, J. Chem. Phys., 2019, 150, 204703], with the permission of AIP Publishing. experimental result extracted from experiments on Ni(14 13 13) in which the steps were poisoned to measure terrace reactivity. This is as expected: (111) surfaces cannot be made entirely free of defects, and these are expected to lead to overestimation of the measured $S_{0}$ when comparing to results for the idealized defect free (111) surface addressed by theory. The results of Fig. 66 give support to the SRP32-vdW1 DF being transferable among $\mathrm{CH}_{4}+\mathrm{Ni}(111)$ and $\mathrm{Ni}(211)$, though experimental verification of the predictions for $\mathrm{MB}$ experiments is still needed for a definite assessment.

5.4.7. $\mathbf{C H}_{4}+\operatorname{Ir}(\mathbf{1 1 1})$. The SRP32-vdW1 DF developed for methane $+\mathrm{Ni}(111)^{44}$ has also been used in RPH calculations on $\mathrm{CH}_{4}+\operatorname{Ir}(111) .{ }^{634}$ The $S_{0}$ computed with the RPH method for $\mathrm{CH}_{4}$ in its vibrational ground state is compared with values measured in laser-off molecular beam experiments (Fig. 67, note that we have added the horizontal displacements between the computed and the measured values in meV). In the calculations the RAA was made, and no attempt was made to describe the effects of the velocity distributions and vibrational state populations associated with the molecular beams used in the experiments. The MD between the experimental data and the computed results that could be determined by digitizing the data in Fig. 3 of ref. 634 was only $0.67 \mathrm{kcal} \mathrm{mol}^{-1}$, and to the eye the comparison between computed and measured results for the initial-state selected reaction probabilities for the $1 \nu_{3}$ state (Fig. 4 of ref. 634) looks even better. It is therefore tempting to call the SRP32-vdW1 DF also an SRP DF for $\mathrm{CH}_{4}+\operatorname{Ir}(111)$. We refrain from doing so because it is not completely clear what the simultaneous effects are of making the RAA and neglecting

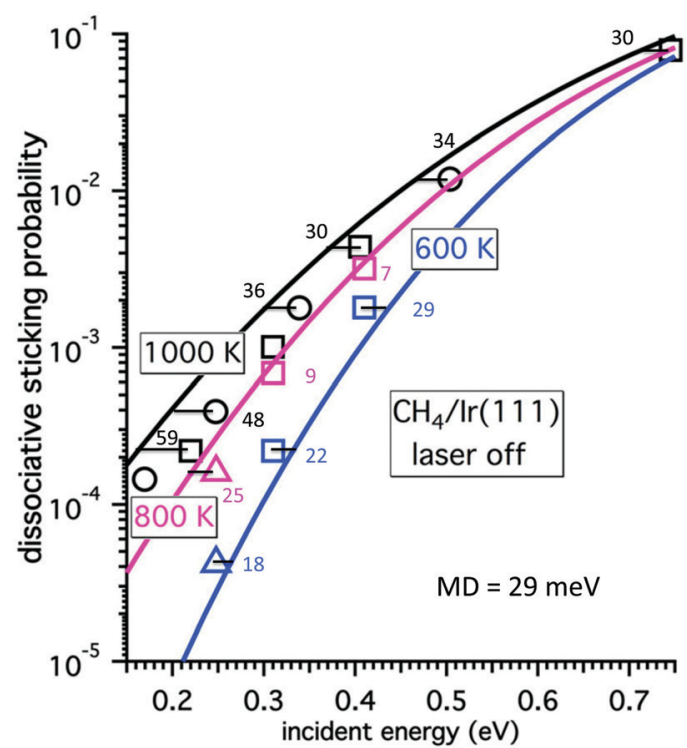

Fig. 67 Comparison of $S_{0}$ computed ${ }^{634}$ with the SRP32-vdW1 DF and the $\mathrm{RPH}$ method for $\mathrm{CH}_{4}+\operatorname{Ir}(111)$, for molecules in the ground vibrational state with experimental values for laser-off conditions (the circles, ${ }^{239}$ squares, ${ }^{733}$ and triangles ${ }^{478}$ ). The temperatures indicate surface temperature (black: $1000 \mathrm{~K}$, magenta: $800 \mathrm{~K}$, blue: $600 \mathrm{~K}$ ). Reprinted from [B. Jackson, Direct and trapping-mediated pathways to dissociative chemisorption: $\mathrm{CH}_{4}$ dissociation on Ir(111) with step defects, J. Chem. Phys., 2020, 153, 034704], with the permission of AIP Publishing. 
the widths of the velocity distributions on the comparisons, and what the effect is of ignoring the contribution of vibrationally excited states in the comparison to the laser-off experiments.

5.4.8. $\mathbf{C H}_{3} \mathrm{OH}+\mathbf{C u}(\mathbf{1 1 1})$. The SRP32-vdW1 DF has also been used in DFMD simulations of sticking of methanol on $\mathrm{Cu}(111)$, using MB parameters deemed representative of $\mathrm{H}_{2}$ seeded beams. ${ }^{183}$ Predictions were made for laser-off reaction and for reaction of $\mathrm{CH}_{3} \mathrm{OH}$ with the $\mathrm{OH}$ stretch $\nu_{1}$ mode pre-excited with one quantum (Fig. 68a). The minimum $E_{\mathrm{b}}$ was found for OH-cleavage ( $92.4 \mathrm{~kJ} \mathrm{~mol}^{-1}$ ), although CH-cleavage is also possible $\left(E_{\mathrm{b}}=130.4 \mathrm{~kJ} \mathrm{~mol}^{-1}\right)$. The computed $S_{0}$ were substantial (0.04-0.17 for laser-off reaction for the $E_{i}$ shown in Fig. 68$) .{ }^{183}$ The occurrence of trapping introduces an uncertainty in the computed $S_{0}$ that however decreases with increasing $E_{i}$ (Fig. 68a). Sticking occurs predominantly through $\mathrm{OH}$-cleavage (Fig. 68b), in accordance with the ordering of the $E_{\mathrm{b}}$ of $\mathrm{OH}$ and $\mathrm{CH}$ cleavage. ${ }^{183}$ The DFMD calculations also looked at formaldehyde formation, and suggested that at high $E_{i}$ this would primarily occur in a mechanism in which $\mathrm{CH}$ cleavage occurs first. ${ }^{183}$ Dissociation of methanol on $\mathrm{Cu}$ surfaces is relevant to steam reforming of methanol. ${ }^{706}$ The $S_{0}$ presented in Fig. 68a may serve as predictions for MB sticking experiments, which would test the

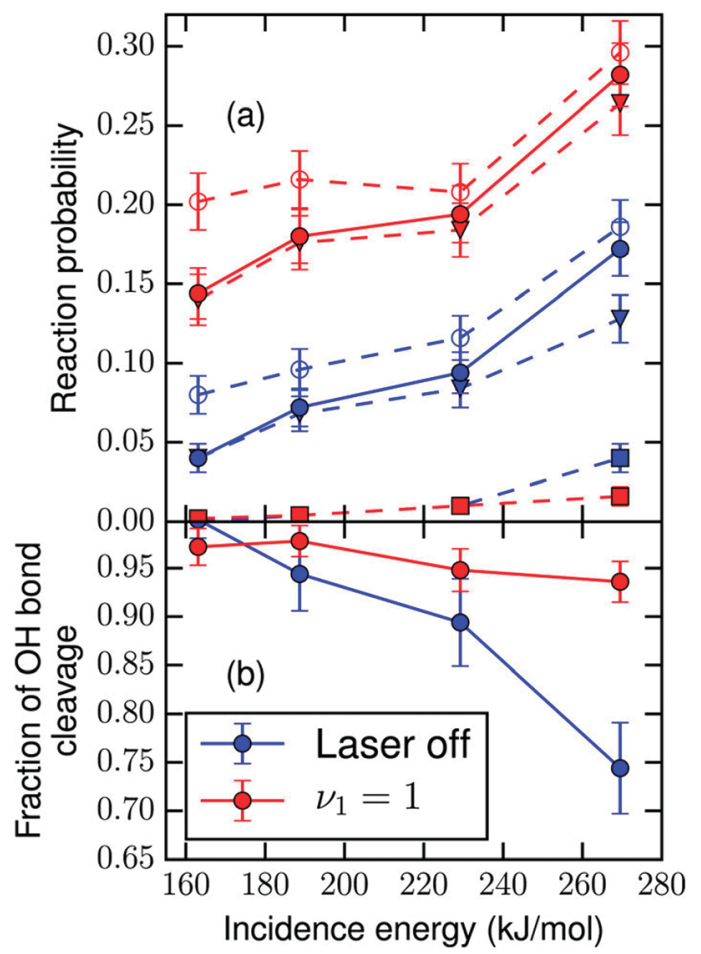

Fig. $68 \mathrm{~S}_{0}$ as computed for $\mathrm{CH}_{3} \mathrm{OH}$ sticking to $\mathrm{Cu}(111)$ in its $\mathrm{OH}$-stretch excited vibrational state (red symbols and lines) and under "laser off" conditions (blue symbols and lines, panel a). ${ }^{183}$ The $S_{0}$ are shown for methanol dissociating via a $\mathrm{CH}$ bond (squares), the $\mathrm{OH}$ bond (triangles), or through any bond (circles, with the empty circles including a contribution from trapping trajectories assuming they all dissociate). The circles in panel $b$ show the fraction of sticking through $\mathrm{OH}$-bond cleavage. Reprinted from [N. Gerrits and G. J. Kroes, An AIMD study of dissociative chemisorption of methanol on $\mathrm{Cu}(111)$ with implications for formaldehyde formation, J. Chem. Phys., 2019, 150, 024706], with the permission of AIP Publishing.

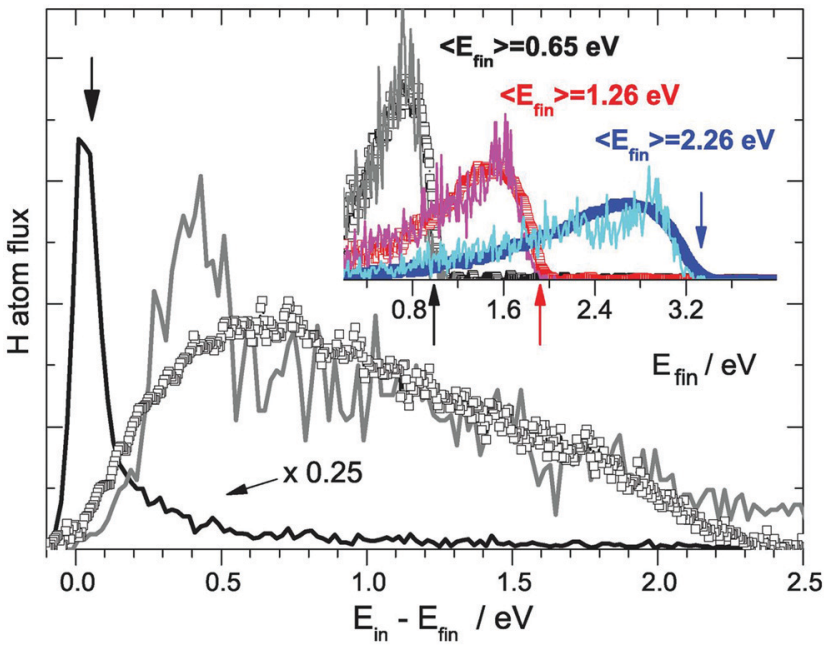

Fig. 69 Comparison of measured and computed kinetic energy loss spectra, for $\mathrm{H}$ scattering from $\mathrm{Au}(111) .{ }^{192}$ In the main figure, results are shown for $E_{i}=2.76 \mathrm{eV}$. The squares represent experimental results, and the curves computational results obtained with electron-hole pair excitation turned on (grey curve) or turned off (black curve) in the model. The insets show measured and computed energy losses for other $E_{i}$ as indicated. From [O. Bünermann, H. Y. Jiang, Y. Dorenkamp, A. Kandratsenka, S. M. Janke, D. J. Auerbach and A. M. Wodtke, Electron-hole pair excitation determines the mechanism of hydrogen atom adsorption, Science, 2015, 350, 1346-1349, https://science.sciencemag.org/content/350/6266/ 1346.long]. Reprinted with permission from AAAS.

degree of transferability of a SRP-DF among systems $\left(\mathrm{CH}_{4}+\right.$ $\mathrm{Ni}(111)$ to $\left.\mathrm{CH}_{3} \mathrm{OH}+\mathrm{Cu}(111)\right)$ in which both the reacting molecule and the metal would differ, although the metals are in adjacent groups in the periodic system.

5.4.9. $\mathbf{H}+\mathbf{A u}(\mathbf{1 1 1})$ and $\mathbf{C u ( 1 1 1 )}$. The SRP48 DF developed for $\mathrm{H}_{2}+\mathrm{Cu}(111)^{175}$ has also been used in DFMD, ${ }^{189,191}$ DFMDEFp, ${ }^{189}$ QCT, ${ }^{180,190}$ and MDEF calculations ${ }^{180,192}$ on scattering of H-atoms from $\mathrm{Cu}(111)$ and $\mathrm{Au}(111)$. The MDEF calculations ${ }^{180,192}$ used an EMT PES fit to SRP48 data. ${ }^{180}$ These calculations test the quality of the SRP48 DF less directly: the EMT fit expression is less accurate for $\mathrm{H}+\mathrm{Au}(111)$ as the CRP and $\mathrm{NN}$ methods are for $\mathrm{H}_{2}$ interacting with metal surfaces. More specifically, SRP48 DFT data were fit to an EMT PES for $\mathrm{H}+\mathrm{Au}(111)$ with an RMS error of about $0.15 \mathrm{eV} \cdot{ }^{180}$ Energy losses in scattering of $\mathrm{H}$ from $\mathrm{Au}(111)$ computed with MDEF using the SRP48 EMT PES were in quite good agreement with experiment, ${ }^{192}$ as shown by Fig. 69. The significance of this observation should not be exaggerated: The quality of the theoretical description owes much to the description of the effects of ehp excitation within the LDFA approach, which is apparently quite accurately done with the EMT densities. ${ }^{180,192}$ It is possible that a similarly accurate MDEF description of the experimental data could have been achieved on the basis of a standard GGA DF such as PBE or RPBE.

\section{Additional discussion}

6.1. For which systems does GGA exchange work, and why?

In Fig. 70 we show for which systems it has been possible to reproduce $\mathrm{MB}$ sticking experiments on DC of small molecules 
on metal surfaces with chemical accuracy, using SRP-DFs or c-SRP DFs containing GGA exchange. We also show for which two systems dynamics calculations using the RPBE DF failed, by overestimating the reactivity $\left(\mathrm{O}_{2}+\mathrm{Al}(111)\right.$, see Fig. 7 , and $\mathrm{HCl}+\mathrm{Au}(111)$, see Fig. 57). The RPBE $\mathrm{DF}^{196}$ can be considered a highly repulsive GGA DF, which however still correctly describes the free electron gas limit. Finally, we also show two systems for which it is doubtful that DFs based on GGA exchange will work (with the reactivity of $\mathrm{NH}_{3}+\mathrm{Ru}(0001)$ overestimated with the RPBE-vdW1 DF (Fig. 58), and the reactivity of $2 \nu_{3} \mathrm{D}_{2} \mathrm{O}$ on $\mathrm{Ni}(111)$ being overestimated with the RPBE DF, see Fig. 24; note that QCT calculations based on a optPBE-vdW HDNNP also overestimated the sticking in $\left.\mathrm{H}_{2} \mathrm{O}+\mathrm{Pt}(110)-(1 \times 2)^{468}\right)$. The results for $\mathrm{O}_{2}+$ $\mathrm{Al}(111)$ are consistent with the finding that dynamics calculations based on GGA DFs consistently overestimate measured probabilities for activated DC of $\mathrm{O}_{2}$ on other metal surfaces, as observed for $\mathrm{O}_{2}+\mathrm{Ag}(111),{ }^{82} \mathrm{Cu}(100),{ }^{497} \mathrm{Cu}(111),{ }^{130,135} \mathrm{Cu}_{1 \mathrm{ML}} / \mathrm{Ru}(0001),{ }^{130,135}$ and $\mathrm{Cu}_{2 \mathrm{ML}} / \mathrm{Ru}(0001){ }^{130}$ Note that in all these studies on $\mathrm{O}_{2}$ dissociation the RPBE DF was used, except for the work on $\mathrm{O}_{2}+$ $\mathrm{Cu}(100)$, which used the PW91 DF. Fig. 70 suggests the following conclusion: For systems for which the difference of the work function of the metal surface $(\Phi)$ and the electron affinity of the molecule (EA) exceeds $7 \mathrm{eV}$, SRP-DFs and c-SRP DFs can successfully be developed on the basis of GGA exchange. For $(\Phi$-EA $)<7 \mathrm{eV}$ efforts to develop a SRP-DF or c-SRP DF based on GGA exchange have sometimes miserably failed, and the chance of success with this approach is unclear.

To explain the dependence of the ability of DFT based on GGA exchange to predict reactivity on metals on ( $\Phi$-EA), we follow Carter and co-workers, who addressed the failure of semi-local DFT for $\mathrm{O}_{2}+\mathrm{Al}(111) .{ }^{399}$ According to them, the lack of derivative discontinuities ${ }^{707}$ and SIE cause semi-local DFT to favor electron delocalization. This is expected to lead to unphysically facile charge transfer from $\mathrm{Al}(111)$ to $\mathrm{O}_{2} \cdot{ }^{399}$ More generally, too facile charge transfer, and therefore too low $E_{\mathrm{b}}$, would be expected for systems for which $(\Phi$-EA) is low. However, there is also a positive message in Fig. 70: DFT based on GGA exchange is clearly capable of an accurate description of DC on metals for systems in which ( $\Phi$-EA) is high enough! This would not have been expected on the basis of DFT results for gas phase reaction barriers ${ }^{318}$ (see Section 2 and Table 1). Fig. 70 suggests that an accurate description is possible with GGA-exchange based SRP-DFs for systems in which $\left(\Phi\right.$-EA) $\geq 7 \mathrm{eV} .^{351}$ Earlier $^{694,708}$ and recent ${ }^{351}$ work suggests that it may also be possible to construct SRP-DFs on systems with low ( $\Phi$-EA) such as $\mathrm{O}_{2}+\mathrm{Al}(111)$ if hybrid DFs are used in the construction of the SRP-DF, but this is yet to be proven.

There is a caveat with the above analysis. The inspiration to plot systems as a function of ( $\Phi$-EA) here comes from work by Wodtke and Auerbach and co-workers, who recognized that $(\Phi$-EA) should correlate with electron transfer (for which the energetics is most favorable for low values of $(\Phi$-EA $)) .{ }^{268}$ However, they correlated ( $\Phi$-EA) mostly with the likelihood that conventional theoretical approaches towards scattering of molecules from metals break down due to a failure to describe electronically non-adiabatic effects. ${ }^{268}$ The idea behind this, which also goes back to the work of Lundqvist and coworkers ${ }^{709}$ who considered the effect of the electronegativity of molecules interacting with $\mathrm{Al}(111)$, is that facile electron transfer to the molecule will also promote electronically non-adiabatic effects. While we cannot completely rule out this explanation, we consider non-adiabatic effects as an unlikely cause for the noted breakdown of GGA-exchange based dynamics approaches for systems with low ( $\Phi$-EA), for two reasons. Firstly, in dynamics calculations ehp excitation based on LDFA friction was found to have only a

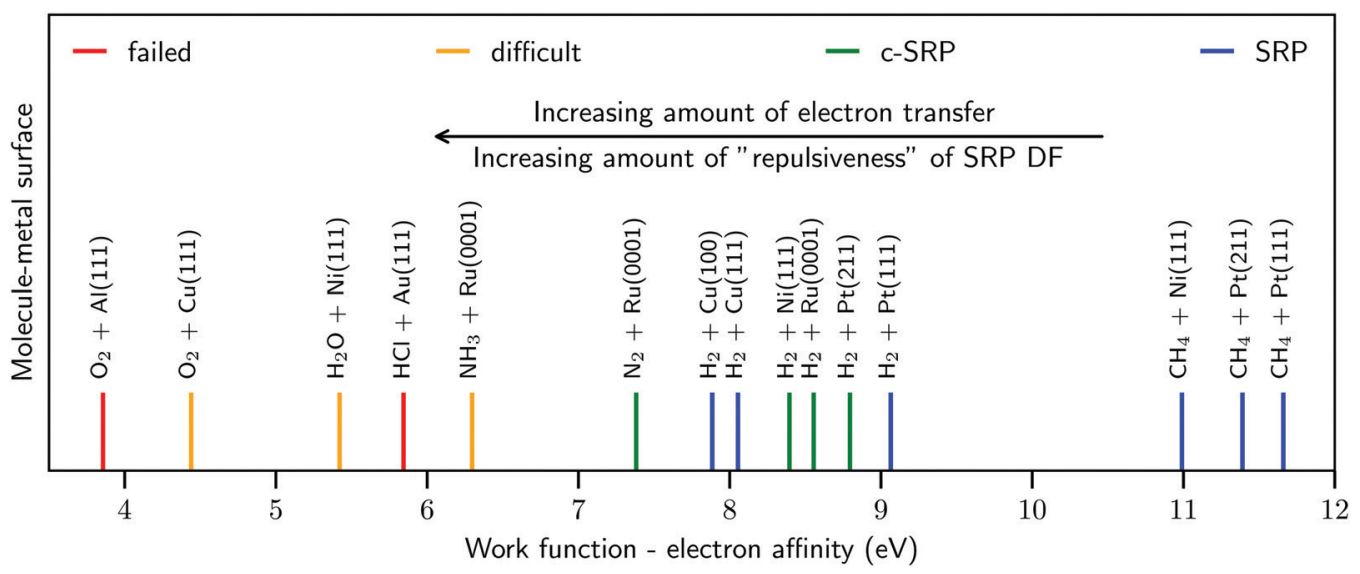

Fig. 70 Correlation of the ability of DFs based on GGA-exchange with the difference between the work function of the metal surface and the electron affinity of the incoming molecule ${ }^{351}$ (shown with vertical lines). The blue and green lines represent systems for which it was possible to derive and SRP or a c-SRP DF, respectively. The red lines represent systems for which the use of the repulsive RPBE DF leads to overestimating computed $S_{0}$, while the orange lines indicate systems for which computed results strongly suggest that this is the case. The work functions for the metal surface were taken from ref. 734, except for the value for Pt(211), which was taken as the value of ref. 734 for Pt(111) plus the difference of calculated LDA values ${ }^{735}$ for Pt(211) and $\mathrm{Pt}(111)$. The electron affinities (EA) are taken from ref. 736 (composite $\mathrm{G} 4$ theory except for the $\mathrm{H}_{2} \mathrm{O}$ value, which was taken from CCSD(T) calculations with the daug-cc-pVTZ basis, and the $\mathrm{HCl}$ value (B97D3 DF with an aug-ccx-pVTZ basis set)), and the value for $\mathrm{CH}_{4}$, for which the electron affinity was taken from calculations on the quartet state of the anion. ${ }^{737}$ Reprinted from ref. 351 (https://pubs.acs.org/doi/10.1021/acs.jpclett.0c02452). Further permission requests to be directed to the ACS. 
small effect on DC of $\mathrm{D}_{2} \mathrm{O}$ on $\mathrm{Ni}(111)^{122}$ and of $\mathrm{HCl}$ on $\mathrm{Au}(111){ }^{147}$ Secondly, electronically adiabatic dynamics calculations based on a PES obtained from CWF theory with DFT embedding ${ }^{35}$ were able to reproduce experiments on $\mathrm{O}_{2}+\mathrm{Al}(111)$ with semiquantitative accuracy (Fig. 7), suggesting that the problem lies with DFT.

This still leaves the question of why DFT based on GGA exchange is successful at modeling DC on metal surfaces if ( $\Phi$-EA) is large. As noted in Section 2, this success would not have been expected on the basis of the performance (systematic under-prediction, see e.g. Table 1) of GGA DFs on $E_{\mathrm{b}}$ for gas phase reactions. It has been argued ${ }^{49}$ that GGA exchange-based DFT is successful at describing DC on metals because the TSs tend to be "late", thereby resembling the final states in which the fragments are chemisorbed. As a result, the good performance of the DF tested (BEEF-vdW) was thought merely to reflect its good performance for chemisorption of molecules to surfaces. ${ }^{49}$ However, GGA-exchange based DFs also show a good performance for a number of systems with early barriers, such as the $\mathrm{H}_{2}+\mathrm{Pt}(111){ }^{156}$ $\mathrm{Pt}(211),{ }^{48} \mathrm{Ru}(0001),{ }^{151}$ and Ni(111) ${ }^{476}$ systems shown in Fig. 70.

We consider the following explanation ${ }^{44}$ to be better: GGAs tend to under-predict $E_{\mathrm{b}}$ for gas phase reactions because they favor electron-delocalization, ${ }^{710-712}$ which generally takes place over several atomic nuclei in gas phase TSs. They tend to perform much better for DC on metals due to error cancellation. The idea is ${ }^{44}$ that the electrons involved in bonding in the TS and coming from the molecule are more delocalized than in the molecule, while the electrons coming from the metal are more localized in the TS than in the metal for high ( $\Phi$-EA), because the electrons are already quite delocalized inside the metal. Here we speculate that for low $(\Phi$-EA) in the TS a (partial) charge transfer of the electrons to the molecule occurs. In this case, the electrons will still be more delocalized in the TS on average, explaining why for low ( $\Phi$-EA) GGA-exchange based DFT breaks down. The reason that GGA DFs break down for charge transfer is that the concept of delocalization is connected with the concept of "fractional charge": ${ }^{707,711,713,714}$ LDA and gradient corrected DFs predict too low energies for effective charges on the nuclei that considerably differ from integer numbers. The explanation offered above is obviously still of a hand-waving nature, and in future efforts will have to be made to provide it with a firm support.

\subsection{Strategies for deriving SRP-DFs, and why SRP-DFT should} work

6.2.1. Starting with a specific generic form of the SRP-DF. An attempt to derive an SRP-DF for a particular system can simply start with writing down a hopefully suitable generic expression for the SRP-DF, with one fitting parameter in it. As described above, experience with early barrier $\mathrm{H}_{2}$-metal surface systems and with $\mathrm{CH}_{4}+\mathrm{Pt}(111)^{157}$ has suggested that the SRP-DF should contain van der Waals correlation. It is probably better to use the vdW2 $2^{327}$ than the vdW $1^{326}$ DF: experience suggests that with vdW1 the van der Waals well depth is overestimated, ${ }^{44,47}$ while the vdW2 DF yields reasonable well depths. ${ }^{156,295}$ Most of our attempts have used GGA exchange DFs in the SRP-DF.
We suggest using a weighted average of the PBE and RPBE exchange DFs in eqn (2c), and resorting to the PBE $\alpha$ DF if PBE exchange is still too repulsive (eqn (2d)). One can also use a SRP-DF based on mGGA exchange and correlation. ${ }^{152}$ Work on $\mathrm{H}_{2}+\mathrm{Cu}(111)$ suggests that such DFs can be highly accurate and show enhanced transferability, but that they are less tunable than SRP-DFs based on GGA exchange. For systems with $(\Phi$-EA $)<7 \mathrm{eV}$ it will probably be necessary to use a screened hybrid exchange $\mathrm{DF}^{351}$ combined with a suitable van der Waals correlation functional, ${ }^{358}$ with optimizing the maximum ratio of exact exchange a potentially viable strategy to arrive at an SRP-DF.

6.2.2. Brute force search for SRP-DFs. A good SRP-DF yields a $S_{0}$ curve for activated dissociation that exhibits the correct reaction threshold and steepness of the $S_{0} v s$. $E_{i}$ curve. DFs that perform well for a similar reaction exhibit a similar minimum $E_{\mathrm{b}}$ and energetic corrugation, as shown for $\mathrm{H}_{2}+\mathrm{Ru}(0001)^{151}$ (Section 5.2.1 and Fig. 48). Figures like Fig. 48 may therefore be used in a brute force search for a SRP-DF for a specific system, as also shown for $\mathrm{H}_{2}+\mathrm{Ni}(111)^{476}$ (Section 5.2.2). In such a search, combinations of different exchange and correlation DFs can be tested, with a useful feature being that DFs employing the same correlation DF but different exchange DFs will typically lie on a line in a plot of the minimum $E_{\mathrm{b}} v s$. the energetic corrugation (see Fig. 48). ${ }^{151}$

6.2.3. Joint experimental-theoretical searches. The joint experimental-theoretical research on $\mathrm{CHD}_{3}+\mathrm{Ni}(111),{ }^{44} \mathrm{Pt}(111),{ }^{47}$ and $\operatorname{Pt}(211)^{47}$ also established a protocol for obtaining SRP-DFs for DC of polyatomic molecules on metals, which was called "reaction barriometry". For sticking of $\mathrm{CH}_{4}$ the protocol involves laser-off and initial state-selected experiments and simulations aimed at reproducing these experiments with the target DF. Elements of the strategy are to aim for conditions where the $E_{i}$ exceeds $E_{\mathrm{b}}^{\mathrm{c}}$ and where $T_{\mathrm{S}}>\Theta_{\mathrm{D}}$ (both to establish the validity of classical mechanics in the DFMD simulations, see also Section 4.1.2). More details are in ref. 47. Advantages of a joint experimentaltheoretical search include the possibility that the theorists can benefit from a carefully documented characterization of the velocity- and internal molecular state-distributions by the experimentalists, which is often omitted in purely experimental papers.

6.2.4. Why SRP-DFT should work: the hole model. An analysis of why SRP-DFT should work can be based on the hole model. ${ }^{45}$ In this model, the sticking probability of a diatomic molecule is given by

$$
\begin{gathered}
S_{0}\left(E_{i}\right)=\int H\left\{E_{\mathrm{tot}}-E_{\mathrm{b}}(X, Y, \theta, \varphi)\right\} \mathrm{d} X \mathrm{~d} Y \mathrm{~d} \cos \theta \mathrm{d} \varphi \\
H(x)=1 \text { if } x \geq 0, H(x)=0 \text { if } x<0
\end{gathered}
$$

in which $E_{\text {tot }}$ is the total (incident translational + internal) energy of the molecule and $H(x)$ is the Heaviside or unit step function. Essentially eqn (35) expresses the idea that, for a given molecular configuration $(X, Y, \theta, \varphi)$, the molecule will dissociate if its total energy exceeds the barrier height in the reduced two-dimensional space associated with $(X, Y, \theta, \varphi)$. An alternative statement of the theory is that any incident energy $E_{i}$ 


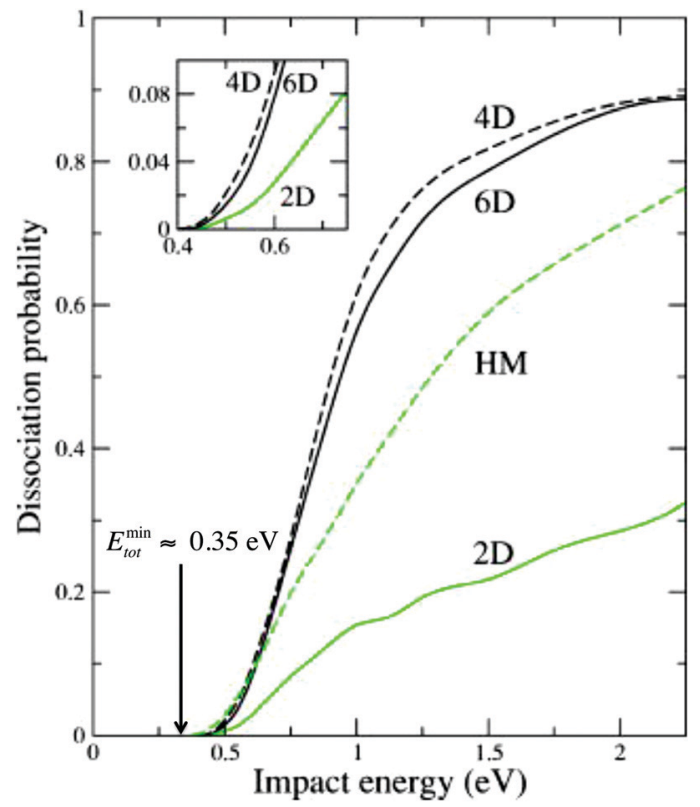

Fig. 71 The dissociative adsorption probability of $(v=0, j=0) \mathrm{H}_{2}$ on $\mathrm{Cu}(110)$ is shown as a function of incidence energy, for normal incidence. ${ }^{715}$ Results are shown of a 2D dynamical model, a 4D dynamical model, and of 6D QCT calculations modeling motion in all molecular degrees of freedom. Also shown are results of using the hole model (HM, see the text). Reprinted from [A. Salin, Theoretical study of hydrogen dissociative adsorption on the $\mathrm{Cu}(110)$ surface, J. Chem. Phys., 2006, 124, 104704], with the permission of AIP Publishing.

and for any rovibrational state $(v, j)$, the sticking probability will be equal to the proportion of configuration space for which $E_{\text {tot }}$ exceeds the barrier height. Fig. 71 (adapted from ref. 715) shows that the hole model (HM) works reasonably well for $\mathrm{H}_{2}+\mathrm{Cu}(110)$ when compared with 6D QCT calculations.

The essential input to the hole model is given by the molecule's internal energy (i.e., its zero-point-vibrational energy for the ground rovibrational state), the minimum barrier to dissociation, and the energetic corrugation of the barrier (the variation of the height of the barrier with $X$ and $Y$ ) and the anisotropy of the barrier height. The main reasons that SRP-DFT works (as now demonstrated for many cases in which $(\Phi$-EA $)>7 \mathrm{eV})$ is that density functionals are capable of giving a good description of the energetic corrugation of the barrier ${ }^{151,177,383,429}$ and of the anisotropy of the barrier height, ${ }^{429}$ that the minimum barrier height can be tuned to the right value, and (trivially) that DFT is capable of computing good vibrational frequencies. The most important evidence that DFT is capable of a correct description of the variation of barrier height with the molecule-surface configuration comes from the DMC calculations on $\mathrm{H}_{2}+\mathrm{Al}(110)$ (see Section 2.4), which suggested that in the absence of a strong attractive van der Waals interaction any semi-local functional is up to the job. Calculations on $\mathrm{H}_{2}+\mathrm{Ru}(0001)$ showed that modeling the van der Waals interaction is essential for the selection of an SRP-DF if the minimum barrier is in the region of the van der Waals well, ${ }^{151}$ while calculations on $\mathrm{CH}_{4}+\mathrm{Pt}(111)$ strongly suggested this to be true if the van der Waals well is deep. ${ }^{157}$
The tunability of the minimum barrier height with semi-local functionals has now been established for several systems with $(\Phi$-EA) $>7 \mathrm{eV}$, i.e., 7 systems for which SRP-DFs have been determined, and 4 systems for which cSRP-DFs have been developed.

Obviously, the hole model is not complete, as several other effects of the PES and dynamical effects are still missing. For example, the main discrepancy between the hole model and the 6D QCT results for $\mathrm{H}_{2}+\mathrm{Cu}(110)$ in Fig. 71 was due to dynamical effects associated with the rotational motion developed by $\mathrm{H}_{2}$ on the way to the barrier. ${ }^{715}$ The fact that SRP-DFT works suggests that semi-local DFT is also capable of accurately describing the more subtle features of the potential energy surface underlying such effects, as demonstrated by the success of DFT with the explanation of several experimental trends observed in dissociative chemisorption studies (see e.g. ref. 29, ref. 30, ref. 43, ref. 264 and ref. 478).

6.2.5. Exploiting transferability. Another useful strategy for developing SRP-DFs may simply be to start with the SRP-DF for a chemically related system, as discussed in the following section.

\subsection{Transferability of SRP-DFs among similar systems and heterogeneous catalysis}

A nice feature of some of the SRP-DFs developed is that they may show transferability among chemically related systems. Examples have been provided for systems in which the same molecule interacts with (i) different low index faces of the same metal (the original SRP-DF for $\mathrm{H}_{2}+\mathrm{Cu}(111)$ also being an SRP-DF for $\mathrm{H}_{2}+\mathrm{Cu}(100),{ }^{46}$ and the optPBE-vdW1 DF being an SRP-DF for $\mathrm{H}_{2}+\mathrm{Cu}(111), \mathrm{Cu}(100)$, and $\mathrm{Cu}(110)^{467}$ ), (ii) low index faces and stepped surfaces of the same metal (the SRP-DF for $\mathrm{CH}_{4}+\mathrm{Pt}(111)$ also being a SRP-DF for $\mathrm{CH}_{4}+\mathrm{Pt}(211),{ }^{47}$ and the SRP-DF for $\mathrm{H}_{2}+\mathrm{Pt}(111)$ being a c-SRP DF for $\mathrm{H}_{2}+\mathrm{Pt}(211)^{48}$ ), (iii) the same low index face of different metals belonging to the same group (the SRP-DF for $\mathrm{CH}_{4}+\mathrm{Ni}(111)$ also being a SRP-DF for $\mathrm{CH}_{4}+\mathrm{Pt}(111)$ ), and (iv) similar low index faces of metals belonging to different groups (PBE-vdW2 ${ }^{195,327}$ is a c-SRP DF for $\mathrm{H}_{2}+\mathrm{Ru}(0001)^{151}$ and $\mathrm{H}_{2}+\mathrm{Ni}(111),{ }^{476}$ and possibly the SRP-DF for $\mathrm{CH}_{4}+\mathrm{Ni}(111)$ and $\mathrm{Pt}(111)$ is an SRP-DF also for $\mathrm{CH}_{4}+$ Ir(111), see Section 5.4.7). Point (ii) above obviously has important implications for heterogeneous catalysis. It suggests that chemically accurate results for a molecule interacting with multifaceted metal nanoparticles with point and extended defects can be obtained by fitting a SRP-DF to a surface science MB sticking experiment addressing the same molecule interacting with a low index face of the metal. ${ }^{47}$

A number of predictions have been made for specific systems based on a SRP-DF for chemically related systems. $S_{0}$ predicted with the SRP-DF for $\mathrm{CHD}_{3}+\mathrm{Ni}(111)$ for $\mathrm{CHD}_{3}+\mathrm{Pd}(111),{ }^{533}$ $\mathrm{CHD}_{3}+\mathrm{Cu}(111),{ }^{63} \mathrm{CHD}_{3}$ colliding with $\mathrm{Cu}(211)$ and single atom surface alloys of Pd and Pt in $\mathrm{Cu}(111),{ }^{185}$ and $\mathrm{CH}_{4}+\mathrm{Ni}(211)^{179}$ all await experimental verification. Such experiments could yield useful additional insights regarding the transferability of SRP-DFs among chemically related systems.

Finally, there are also examples in which the transferability of a SRP-DF to a chemically related system does not hold. Examples discussed above include the lack of transferability of 
SRP-DFs from $\mathrm{H}_{2}+\mathrm{Pt}(111)$ to $\mathrm{H}_{2}+\mathrm{Ni}(111),{ }^{476}$ from $\mathrm{H}_{2}+\mathrm{Ru}(0001)$ to $\mathrm{H}_{2}+\mathrm{CO} / \mathrm{Ru}(0001),{ }^{188}$ and from $\mathrm{H}_{2}+\mathrm{Cu}(111)$ to $\mathrm{H}_{2}+$ $\operatorname{Ag}(111)^{152,186}$ and to $\mathrm{H}_{2}+\mathrm{Au}(111) .{ }^{23,187}$ There are indications that an SRP-DF constructed on a higher DFT rung may show increased transferability. ${ }^{152}$ More knowledge is required concerning under what conditions SRP-DFs are transferable among related systems.

\subsection{Performance of general purpose DFs}

DFs that have been cast as being "general purpose" are the GGA $\mathrm{PBE}^{195} \mathrm{DF}$, and the mGGA revTPSS ${ }^{199}$ DF. These two DFs have both been tested in calculations on $\mathrm{H}_{2}+\mathrm{Ru}(0001)$ (Fig. 1) ${ }^{151}$ and on $\mathrm{H}_{2}+\mathrm{Cu}(111)$ (Fig. 36). ${ }^{152}$ In both cases the revTPSS DF results agreed better with experiment than the PBE results (Fig. 1 and 36). Unlike one might expect the maximally constrained and popular mGGA SCAN ${ }^{339}$ DF shows a worse performance than PBE on $\mathrm{H}_{2}+$ $\mathrm{Cu}(111)$ (Fig. 36). ${ }^{152}$ The observation of the poor performance of the SCAN DF for $E_{\mathrm{b}}$ for DC on metals is in line with findings of others that SCAN performs poorly for chemisorption on metals $^{307,716,717}$ (see also Table 2 and its discussion in Section 2.1.4).

\subsection{Challenges facing SRP-DFT}

6.5.1. Extending the database with systems defying an accurate description so far. As discussed in Section 6.1, so far it has not been possible to develop SRP-DFs for systems with a low value of ( $\Phi$-EA), like $\mathrm{O}_{2}+\mathrm{Al}(111), \mathrm{HCl}+\mathrm{Au}(111), \mathrm{H}_{2} \mathrm{O}+$ $\mathrm{Ni}(111)$, and $\mathrm{NH}_{3}+\mathrm{Ru}(0001)$. It will obviously be a challenge to develop SRP-DFs for these systems, even though reasonably accurate experimental results are available for sticking to compare with. The problem here is that the use of GGA exchange DFs in the construction of the SRP-DFs may not suffice. One may have to go to mGGA exchange of even to $\mathrm{SH}$ DFs, which makes DFT calculations of PESs or on the fly dynamics calculations more expensive. One also has to reckon with the possibility that the sticking is affected by electronically nonadiabatic effects. ${ }^{268}$ It will be a challenge to extend our "database" (the 11 systems for which accurate $E_{\mathrm{b}}$ are available, Sections 5.1 and 5.2) with systems with low ( $\Phi$-EA). We do deem this to be a necessary development, as the "database" now available (as well as the SBH10 database, ${ }^{301}$ which also only contains results for $\mathrm{H}_{2}, \mathrm{~N}_{2}$, and $\mathrm{CH}_{4}$ interacting with metals) may be viewed as "biasing": The absence of systems with low ( $\Phi$-EA) probably results in DFs incorporating GGA exchange being favored over hybrid DFs.

6.5.2. Inaccurate, ill-described, and missing experiments. A semi-empirical approach will in principle not yield higher accuracy than the experiment it was fitted to. The presence of differing results for the same system therefore poses problems to SRP-DFT. $S_{0}$ for highly activated DC may show a strong dependence on the velocity distribution of the molecules in the MBs used, as found for $\mathrm{H}_{2}+\mathrm{Cu}(111)$ (see Fig. 31). ${ }^{43,159}$ Fortunately, $S_{0}$ for weakly activated dissociation show a weaker dependence on this distribution, although deviations may be substantial for high $E_{i}$, as found for $\mathrm{H}_{2}+\mathrm{Ni}(111)$ and $\mathrm{Pt}(111)$ (Fig. 43 and 49). This problem worsens if the experimentalists have not documented the MB parameters, and one has to resort to guess work. More accurate and/or better documented experiments are needed for sticking in $\mathrm{D}_{2}+\mathrm{Pt}(111), \mathrm{H}_{2}+\mathrm{Ni}(111), \mathrm{H}_{2}+\mathrm{Ag}(111), \mathrm{H}_{2}+\mathrm{Pd}(111)$,
$\mathrm{H}_{2}+\mathrm{CO} / \mathrm{Ru}(0001), \mathrm{N}_{2}+\mathrm{W}(110)$, and $\mathrm{N}_{2}+\mathrm{Ru}(0001)$ (Section 5). Appropriate additional experiments on $\mathrm{H}_{2}+\mathrm{Ni}(111)$ and $\mathrm{H}_{2}+$ $\mathrm{Pt}$ (211) might help confirm that the candidate SRP-DFs developed are actual SRP-DFs for these systems.

6.5.3. Making accurate predictions for diffraction. Diffractive scattering in $\mathrm{H}_{2}+\mathrm{Pt}(111)$ and $\mathrm{H}_{2}+\mathrm{Ru}(0001)$ has not yet been described well with the SRP-DF and c-SRP DF for these systems, which in both cases contained a vdW-DF correlation DF (Sections 5.1.3 and 5.2.1). The problem may be due to the DW extrapolation going wrong in the case of multiple scattering in the van der Waals well, although the problem noted for $\mathrm{H}_{2}+$ $\mathrm{Ru}(0001)$ may also be due to the neglect of static surface disorder in the calculations ${ }^{148}$ (Section 5.1.3). Strategies to obtain a good description of diffraction include abandoning the static surface approximation, using an optical potential to describe the attenuating effect on diffraction of multiple scattering in the van der Waals well, and removing the van der Waals well from the PES in a clever way. ${ }^{148}$ These methods have yet to be tried with an SRP-DF.

6.5.4. Other experiments that are not yet not yet well described with SRP-DF. The biggest puzzle remaining for $\mathrm{H}_{2}+$ $\mathrm{Cu}(111)$ is that the probability for vibrational excitation of $\mathrm{H}_{2}$ scattering from $\mathrm{Cu}(111)$ is under-predicted by a factor 2 to 3 with SRP-DFs (Section 5.1.1 and Fig. 35). Calculations suggest that this is not due to neglecting phonons or ehp excitation. ${ }^{168}$ The cause of the problem is unclear. Additional experiments on this system, which would ideally provide state-to-state scattering probabilities $P\left(v, j \rightarrow v^{\prime}, j^{\prime}\right)$ (the experiments now available only yield results summed over $j^{581}$ ), would be helpful.

A more subtle trend not yet reproduced by calculations employing SRP-DFs concerns the $j$-dependence of $E_{0}(v, j)$ for $\mathrm{H}_{2}+\mathrm{Cu}(111)$, i.e., the experimental observation that the $E_{0}(v, 1 j)$ first increase and then decrease with $j$ (see e.g. Fig. 33A). This trend can perhaps be reproduced with calculations also modeling surface atom motion and ehp excitation, if these are done with an accurately fitted (e.g. HDNN) PES. Early work ${ }^{718}$ using a site-dependent LEPS PES ${ }^{492}$ based on un-converged DFT data for $\mathrm{H}_{2}+\mathrm{Cu}(111)^{719}$ did recover the experimental trend in $E_{0}(v, j) .{ }^{641}$ However, because the PES was loosely based ${ }^{492}$ on DFT data that were converged to only $0.2 \mathrm{eV},{ }^{698}$ it is not clear what the significance of this result ${ }^{718}$ was. Another observable from associative desorption experiments performed at high $T_{\mathrm{s}}$ that was not yet reproduced with dynamics calculations using SRP potentials is the ADE of the molecule desorbing in a specific rovibrational state (see Fig. 33B for $\mathrm{H}_{2}+\mathrm{Cu}(111)^{159}$ and Fig. 38 for $\left.\mathrm{H}_{2}+\mathrm{Cu}(100)^{46}\right)$. This will probably require calculations in which the surface atoms are allowed to move, and may require the modeling of ehp excitation as well. Additionally, the measured ${ }^{567}$ probabilities for rotationally elastic and inelastic scattering, and for vibrationally inelastic scattering of $(v=1, j=1)$ $\mathrm{H}_{2}$ from $\mathrm{Cu}(100)$ have not yet been reproduced with SRP-DF based dynamics calculations. ${ }^{46}$

6.5.5. The description of the metal. GGA DFs (or more generally DFs constructed from GGA exchange DFs) that perform well on surface adsorption are unlikely to perform well at describing the metal ${ }^{314,320}$ (Section 2.1.3 and Fig. 5). This may 
be a problem for simulating reaction at high $T_{\mathrm{s}}$, where $S_{0}$ may be affected by surface expansion, ${ }^{172,173}$ which then needs to be described correctly. Emphasis on the correct description of the metal is also needed when modeling DC on stepped and kinked surfaces, e.g. in sticking of $\mathrm{CHD}_{3}$ on $\operatorname{Pt}(110)-(2 \times 1)^{182}$ and on $\operatorname{Pt}(210)^{181}$ (Fig. 59 and 60). Improved results for the latter system can perhaps be obtained on the basis of mGGA-DFs of the MS type, results for $\mathrm{H}_{2}+\mathrm{Cu}(111)$ showing that these may allow the construction of SRP-DFs that also give an excellent description of metallic properties. ${ }^{152}$

6.5.6. Reaction accompanied by trapping. If the sticking is accompanied or even mediated by trapping, this may lead to several problems with methods based on quasi-classical dynamics, like the DFMD and QCT methods. One problem is that it is expensive to perform long trajectory calculations with DFMD, so that it may not be possible to converge the computed reaction probability with respect to the maximum propagation time. This has affected DFMD calculations on $\mathrm{CHD}_{3}$ sticking to $\operatorname{Pt}(110)-(2 \times 1)$ (Fig. $\left.59^{182}\right)$, to $\operatorname{Pt}(210)$ (Fig. 60), ${ }^{181}$ and to $\operatorname{Pt}(211)$ at low $E_{i},{ }^{47}$ and on $\mathrm{N}_{2}$ sticking to $\mathrm{W}(110) .{ }^{88,149}$ One can perhaps circumvent this problem by fitting a HDNNP for the system and performing QCT calculations. This should allow an extension of the maximum propagation time by at least three orders of magnitude. However, another problem is due to the QCT methodology itself. The QCT method may give wrong results for the probability of trapping mediated reaction, as the trapping allows more time for artificial conversion of zpe to motion along the reaction coordinate. ${ }^{590}$ This may result in too much reaction, or even in too little reaction if this artificial energy transfer interferes with a steering mechanism promoting reaction. ${ }^{590}$ This problem may have affected QCT calculations on sticking of $\mathrm{H}_{2}$ to $\operatorname{Pt}(211)^{48}$ (Fig. 50 and Section 5.2.3). Perhaps this problem can be alleviated with the HDNNP approach as done earlier in 5D dynamics calculations on trapping mediated DC of $\mathrm{H}_{2},{ }^{590}$ i.e., by incorporating the zpe energy in the potential and performing so-called CZPE calculations (Section 4.2).

6.5.7. Systems with deep molecular chemisorption wells. Systems exhibiting both molecular chemisorption and DC, like $\mathrm{N}_{2}+\mathrm{W}(110)^{149,512}$ and $\mathrm{N}_{2}+\mathrm{W}(100),{ }^{143,512}$ may pose special problems. In these systems, the balance between DC and molecular chemisorption needs to be correctly described. A potential problem is that the best DF describing $E_{\mathrm{b}}$ is not necessarily the best DF describing molecular chemisorption, just like the best DF describing gas phase $E_{\mathrm{b}}$ is not necessarily the best DF describing gas phase reaction energies. ${ }^{689}$ One way to circumvent this problem might be to construct an SRP-DF on the highest DFT rung possible. Unfortunately trapping in molecular chemisorption wells may exacerbate this problem (Section 6.5.6).

6.5.8. The importance of using the correct dynamical method. An obvious question to ask is whether the use of quasi-classical dynamics suffices, or whether QD calculations are required. For the DC of diatomic molecules the answer is easiest: even for activated dissociation of $\mathrm{H}_{2}$, quasi-classical dynamics will usually be highly accurate for simulating a $\mathrm{MB}$ experiment on sticking (see e.g. Fig. 16). The reason is that at low $E_{i}$ reaction will be dominated by the vibrationally excited $\mathrm{H}_{2}$ present in the beam, for which the QCT method is accurate.

For the reaction of polyatomic molecules on metal surfaces the question of which dynamics method one should use is harder to answer. It remains a considerable challenge to perform QD calculations on DC of polyatomic molecules on metal surfaces, even for the smallest conceivable covalent triatomic molecule, i.e., $\mathrm{H}_{2} \mathrm{O}$. While 9D QD calculations have been realized for $\mathrm{H}_{2} \mathrm{O}$ dissociating on $\mathrm{Cu}(111)^{61}$ and $\mathrm{Ni}(100)^{98}$ within the static surface approximation, no such calculations have been presented for the $\mathrm{D}_{2} \mathrm{O}$ isotopologue, whereas experimental results are only available for $\mathrm{D}_{2} \mathrm{O}+\mathrm{Ni}(111) .{ }^{78}$ However, for the latter system it is possible to perform 7D fixed site QD calculations and use the SAED procedure to get approximate 9D QD results, and to get $T_{\mathrm{S}}$ dependent results using the a posteriori LRS approach. ${ }^{120}$

Given the computational expense of QD, it would obviously be nice if it were sufficient to perform QCT calculations on DC of polyatomic molecules. Unfortunately, the comparison made by Jiang and co-workers for $\mathrm{D}_{2} \mathrm{O}+\mathrm{Ni}(111)^{597}$ suggests that this may be troublesome, due to zpe leakage (Fig. 20). It remains to be established if and to what extent this problem is specific to $\mathrm{D}_{2} \mathrm{O}+\mathrm{Ni}(111)$, and to what extent it can be solved for this and other systems by using the RPMD method. ${ }^{597}$ One may obviously wonder whether the same problem should exist for $\mathrm{CH}_{4}$ dissociation on metal surfaces. In this sense it is encouraging that TST calculations on methane dissociation on Ni(111) using the SRP-DF for this system are in excellent agreement with experiment for sticking under thermal conditions ${ }^{178}$ (Section 5.4.6 and Fig. 66). This suggests that the DFMD procedure to extract an SRP-DF for the system investigated worked, and that the quasi-classical procedure employed posed no problems (Section 5.4.6). Nevertheless, one should keep in mind that the QCT method (as effectively used in DFMD) is probably not accurate for MBs with high $T_{\mathrm{N}}$, due to the higher population of vibrationally excited states of methane in the beam. Note in this context that $\mathrm{CHD}_{3}$ was used in much of the DFMD and QCT calculations because the energy put in the $\mathrm{CH}$-stretch mode remains there for a long enough time to perform accurate calculations on sticking of vibrationally pre-excited $\nu_{1}=1 \mathrm{CHD}_{3} .^{51}$

Our conclusion that the DFMD and QCT approaches are probably appropriate for methane DC on metals is a fortunate one in the sense that QD calculations on methane DC can presently not be relied on to yield quantitative accuracy: The $C_{3 \mathrm{v}}$ symmetry restriction imposed on the remaining $\mathrm{CH}_{3}$ fragment may lead to errors of up to $50 \%$ in the $S_{0}$ of $\mathrm{CH}_{4}$ in its vibrational ground state, and to larger errors for excited vibrational states ${ }^{632}$ (Section 4.4.3). There is an additional factor four uncertainty that results from not knowing $^{529}$ whether one may simply multiply the $S_{0}$ computed with the corresponding model, in which only one $\mathrm{CH}$-bond is allowed to break, by this factor. Possibly, full-dimensional QD calculations on methane dissociation on metals will become possible in the near future due to methodological improvements now being made to the MCTDH method. ${ }^{633,720-723}$ Another possibility is that RPMD makes good on its promise, as suggested by the recent results on $\mathrm{H}_{2}+\mathrm{Cu}(111)$ (Fig. 15) and $\mathrm{D}_{2} \mathrm{O}+$ $\mathrm{Ni}(111)$ (Fig. 20), ${ }^{597}$ but also see the critical discussion of these 
results in Section 4.5 concerning the failure of RPMD to accurately describe the QD results at higher $E_{i}$.

\subsection{The importance of using the correct dynamical model}

An important point is that, when evaluating the accuracy and predictive value of an SRP-DF for a specific system and observable, one should obviously make sure that one is using the correct dynamical model. Examples provided above include that one should use the BOMS model when computing $A_{0}^{(2)}(J)$ for $\mathrm{D}_{2}$ desorbing from $\mathrm{Cu}(111)^{175}$ (Fig. 34 and Section 5.1.1). Work on $\mathrm{H}_{2}$ dissociation on metal surfaces at elevated $T_{\mathrm{s}}$ also suggests that one should take into account the effect that metal lattice expansion, including interlayer relaxation, may have on the barrier to dissociation. ${ }^{172,173}$ This is often overlooked in calculations on DC on hot surfaces.

In calculations on vibrational excitation or de-excitation, in for instance $\mathrm{H}_{2}+\mathrm{Cu}(111)$ (and $\mathrm{HCl}+\mathrm{Au}(111)$ ), it will probably ${ }^{168}$ (almost certainly ${ }^{220}$ ) be important to model electronically nonadiabatic effects. Here it may also be important which EF method is used to describe ehp excitation, if a EF-based approach is selected (one may also choose the IESH method ${ }^{128,575}$ ). Calculations using ODF and the LDFA to obtain friction coefficients yield different results for vibrational de-excitation from $v=2$ to $v=1$ in scattering of $\mathrm{H}_{2}$ from $\mathrm{Cu}(111),{ }^{57}$ and for sticking and vibrational excitation of $\mathrm{N}_{2}$ on $\mathrm{Ru}(0001)$ (Fig. 14 and 51). ${ }^{59}$ New experiments are needed to test these predictions. Also, additional tests of the LDFA and ODF approaches, especially dynamics calculations comparing with existing experiments such as on $\mathrm{HCl}$ dissociation on $\mathrm{Au}(111)^{219}$ (Fig. 57), are expected to be useful.

For DC of $\mathrm{H}_{2}$ on $\mathrm{CO} / \mathrm{Ru}(0001)$ the selected BOMS model should be adequate, but a question remains about the size of the surface unit cell used. ${ }^{188}$ For this and other systems ${ }^{118,501,532,538,539}$ in which a molecule dissociates on a surface partly pre-covered by other molecules, ${ }^{42,631}$ it may be useful to attempt the development of HDNNPs. In principle HDNNPs should allow accurate dynamics calculations using larger surface unit cells than manageable in DFMD.

\subsection{Interpretation of dynamical effects in DC}

An important point is that calculations using SRP-DFs may obviously lead to important insights in the reaction dynamics of the system investigated. Examples of useful insights derived in studies using SRP-DFs are plentiful, and here we just mention a few. Calculations using the BOMS model showed that increasing $T_{\mathrm{s}}$ leads to decreased $A_{0}^{(2)}(J)$ for $\mathrm{H}_{2}$ desorbing from $\mathrm{Cu}(111)$, in improved agreement with experiment, part of the reason being that the accompanying surface thermal expansion leads to lower $E_{\mathrm{b}} \cdot{ }^{175}$ Studies modeling vibrational excitation of $\mathrm{H}_{2}$ scattering from $\mathrm{Cu}(111)$ have been able to attribute the measured increase of vibrational excitation with $T_{\mathrm{s}}$ to ehp excitation rather than a mechanism involving phonons. ${ }^{168}$ DFMD on DC of methane on $\mathrm{Ni}(111)^{44}$ suggests that the reaction mechanism is closer to rotationally adiabatic than to rotational sudden, and this and $\mathrm{s}$ imilar findings of earlier AIMD simulations on $\mathrm{CHD}_{3}+$ $\operatorname{Pt}(111)^{51}$ have been used to increase the accuracy of RPH calculations. ${ }^{89,178,179}$ Dynamics calculations using SRP-DFs for the specific system studied or for a chemically related system have provided useful insights into why the presence of steps may ${ }^{4,165}$ or may not ${ }^{166}$ promote sticking in specific cases.

We are not saying that such insights could not have been obtained in dynamics studies using standard DFs, like PBE. ${ }^{195}$ However, it is easier to attach belief to a mechanism revealed by or insight obtained from a dynamics study in which it was also possible to reproduce the measured $S_{0}$ to within chemical accuracy, and some computed observables are quite sensitive to the details of the PES used. ${ }^{117}$

\subsection{Predictions from SRP-DFT}

We close this Section by noting that a number of predictions have been made with SRP-DFs, for systems that are chemically related to varying extent to systems for which the SRP-DF was developed. Systems for which we hope experiments will be done include $\mathrm{CHD}_{3}+\operatorname{Pd}(111)$ (Fig. 64), ${ }^{533} \mathrm{CHD}_{3}+\mathrm{Cu}(111)$ (Fig. 9), ${ }^{63}$ $\mathrm{Cu}(211),{ }^{185}$ and single atom surface alloys of $\mathrm{Cu}(111),{ }^{185} \mathrm{CH}_{4}+$ $\mathrm{Ni}(211),{ }^{178,179} \mathrm{CH}_{3} \mathrm{OH}+\mathrm{Cu}(111)$ (Fig. 68), ${ }^{183}$ and $\mathrm{HOD}+\mathrm{Ni}(111)$ (Fig. 63). ${ }^{184}$ Such experiments will yield additional information about the transferability of SRP-DFs among chemically related systems.

\section{Summary and conclusions}

To be able to compute accurate rates of heterogeneously catalyzed processes we need more accurate electronic structure methods than now available through standard DFT at the GGA and mGGA levels, especially for the calculation of $E_{\mathrm{b}}$ for DC on metals. ${ }^{10,301}$ An accurate description of the molecule-metal surface interaction is also needed for evaluating how the initial vibrational, rotational, and translational energy of the molecule, its initial alignment or orientation with respect to the surface, surface temperature, surface phonons, and ehp excitation may affect the sticking. Novel applications of electronic structure methods that are in principle more accurate than DFT, like $\mathrm{DMC}^{34}$ and the $\mathrm{ECW}^{35,392,399}$ method, are emerging but have not yet delivered the accuracy in $E_{\mathrm{b}}$ for DC that is desired (chemical accuracy, i.e., $1 \mathrm{kcal} \mathrm{mol}^{-1}$ ). Fortunately, accurate reaction barriers for DC on metal surfaces may be extracted through a dynamical approach based on SRP-DFT, ${ }^{10,43,44,47,59}$ which is a semi-empirical method. In this procedure, the $E_{\mathrm{b}}$, which is not a direct observable but is computed with a specific $\mathrm{DF}$, is assumed to be accurate if the $S_{0}\left(E_{i}\right)$ curve computed with that DF with an adjustable parameter in it is shifted from the $S_{0}\left(E_{i}\right)$ curve measured in a MB experiment by no more than 1 kcal mol ${ }^{-1} \cdot{ }^{43}$ Many surface reaction dynamics studies still use standard DFs for the molecule-surface interaction, and we have provided examples throughout this review illustrating the limited accuracy available through such DFs for surface reactions of e.g. $\mathrm{H}_{2}, \mathrm{~N}_{2}, \mathrm{CH}_{4}$, and $\mathrm{H}_{2} \mathrm{O}$. However, increasingly SRP-DFs are being developed, and a database of chemically accurate $E_{\mathrm{b}}$ for DC on metals is emerging. ${ }^{10,43,44,46-48,59,151,156,467,476}$

Standard DFs now used in dynamics calculations on DC on metals are usually taken at the semi-local GGA rung of DFT (see the Introduction), while calculations based on mGGA DFs are 
starting to emerge. ${ }^{151,152,351,695}$ Hybrid DFs are still expensive to use with dynamics calculations, but applications are emerging in which screened hybrid functionals are used. ${ }^{351}$ The performance of standard DFs on adsorption of molecules and atoms to metals and on $E_{\mathrm{b}}$ for DC of molecules on metals has been compared to their performance on $E_{\mathrm{b}}$ for gas phase reactions for the databases discussed in Section 2 (see this section for details and references), including the SBH10 database ${ }^{49}$ containing $E_{\mathrm{b}}$ for DC on metals.

Well-known non-empirical, constraint based GGA (rung 2) DFs include the PW91 ${ }^{312}$ DF and its successor, PBE, ${ }^{195}$ and the RPBE $^{196}$ DF. Semi-empirical GGA and NGA DFs include the MOHLYP $^{315}$ and GAM ${ }^{317}$ DFs and the BEEF-vdW ${ }^{304}$ DF, where the last DF is an example of a DF combining a GGA exchange $\mathrm{DF}^{304}$ with a non-local correlation DF. ${ }^{327}$ This combination enables a reasonably accurate description of the attractive van der Waals interaction, which is not modeled with standard semi-local and hybrid DFs. As discussed in Section 2.1 (see also Tables 1-3) a puzzling finding has been that GGA-exchange based DFs tend to perform quite well on DC of $\mathrm{H}_{2},{ }^{43,46,156} \mathrm{~N}_{2},{ }^{59}$ and $\mathrm{CH}_{4}{ }^{44,47}$ on metal surfaces, although they systematically underestimate $E_{\mathrm{b}}$ for gas phase reactions. ${ }^{31,289}$ In particular, the BEEF-vdW functional showed a mean signed error (MSE) of only $0.7 \mathrm{kcal} \mathrm{mol}^{-1}$ in $E_{\mathrm{b}}$ for DC on metals for the SBH10 database, with the MAE of $2.8 \mathrm{kcal} \mathrm{mol}^{-1}$ however indicating that this DF cannot yet be relied upon for delivering chemical accuracy. ${ }^{301}$

In mGGA (rung 3) DFs the energy also depends on the kinetic energy density $\tau$, which allows one to determine the nature of bonding (metallic, covalent, or weak) in particular regions, ${ }^{340}$ which has been used in the construction of made simple (MS) functionals. ${ }^{344}$ Adding $\tau$ has allowed bringing the MUE in gas phase $E_{\mathrm{b}}$ for the B76 database down from $5.3 \mathrm{kcal} \mathrm{mol}^{-1}$ for the best semi-empirical NGA (i.e., GAM) ${ }^{317}$ to $1.7 \mathrm{kcal} \mathrm{mol}^{-1}$ for the semi-empirical MN15-L meta-NGA DF. ${ }^{32}$ Hybrid (rung 4) DFs also contain exact exchange, the calculation of which scales unfavorable with the number of electrons as it requires a double integral over $3 \mathrm{D}$ space. This can be somewhat alleviated by using SH DFs; these DFs are just starting to get used in dynamics calculations on DC on metals. ${ }^{351}$ The semiempirical SH mGGA DF MN12-SX ${ }^{357}$ showed a MUE of only $1.15 \mathrm{kcal} \mathrm{mol}^{-1}$ for gas phase $E_{\mathrm{b}}$ for the B76 database, ${ }^{289}$ but has to our knowledge not yet been tested on $E_{\mathrm{b}}$ for DC on metals. For the SBH10 database for DC on metals, the mGGA MS2 and the HSE06 DFs were both out-performed by the GGA-NLD BEEF-vdW DF. ${ }^{301}$

Rung 5 DFs also use the virtual Kohn-Sham orbitals to calculate the correlation energy with a formalism that is $\mathrm{NL}$ in the orbitals. The most important example of this class, the RPA, ${ }^{359-362}$ has a MUE of only $2.3 \mathrm{kcal} \mathrm{mol}^{-1}$ for the B76 database of gas phase $E_{\mathrm{b}},{ }^{349}$ and of $4.8 \mathrm{kcal} \mathrm{mol}^{-1}$ for the CE10 database of chemisorption energies on metals. ${ }^{306}$ While this may be considered to be a good performance, the RPA is not accurate enough to yield benchmark results to use as a yardstick to measure $E_{\mathrm{b}}$ for DC on metals against. This situation is different from that which exists for the evaluation of DFs on their performance for gas phase systems, which can be made by a direct comparison with accurate theoretical, i.e., $\operatorname{CCSD}(\mathrm{T})$ results. ${ }^{31,289}$ At this stage the performance of DFs on DC on metals can therefore be evaluated only by computing $S_{0}\left(E_{i}\right)$ curves for DC on metals and comparing these to results from MB sticking experiments. ${ }^{10}$

To enable chemically accurate reaction barriers to be extracted from such comparisons, the SRP-DFT procedure has been devised. ${ }^{10,43}$ So far, SRP DFs have been constructed as a weighted average of two GGA DFs, ${ }^{43,46}$ as a weighted average of two GGA exchange DFs and a van der Waals correlation functional, ${ }^{44,47}$ as a combination of a tunable GGA exchange DF and a van der Waals correlation $\mathrm{DF},{ }^{156}$ and it has been shown that they can be constructed as a weighted average of two mGGA DFs. ${ }^{152}$ A DF may be called an SRP DF if it also allows a different experiment to be quantitatively reproduced than the experiment it was fitted to for the specific system considered. ${ }^{10,43}$ If a DF only reproduces the $S_{0}$ measured in the MB experiment it was fitted to for the specific system considered $^{48,59,151,476}$ it is called a c-SRP DF for that system. First principles electronic structure methods that show a promise of high future accuracy are the ECW method of Carter and co-workers, ${ }^{392}$ which has been applied to e.g. $\mathrm{O}_{2}+$ $\operatorname{Al}(111),{ }^{35,399}$ and the DMC method, ${ }^{414,415}$ which has been applied to e.g. $\mathrm{H}_{2}+\mathrm{Cu}(111){ }^{34}$

Reaction barrier heights are not directly observable, and, for DC on metals, can only be reliably extracted from comparisons with MB sticking experiments. ${ }^{10}$ This requires dynamics calculations, which can be done most efficiently if a PES is available. At the same time, such a comparison only allows clear conclusions regarding the accuracy of the underlying electronic structure method if the underlying fit or interpolation of the PES is accurate. Examples of highly accurate fitting methods are the PIP-NN method for molecules interacting with static surfaces, ${ }^{439,440}$ and the HDNN method for molecules interacting with mobile surfaces. ${ }^{432,441-443,466}$ Methods of medium to high accuracy are the $\mathrm{CRP},{ }^{472}$ which is an accurate interpolation procedure for diatomic molecules interacting with static surfaces, and the RFF method, ${ }^{477}$ which is a fitting method for molecules interacting with mobile surfaces. With the possible exception of the RFF method all of the above methods yield PESs in which the electronic structure data are fitted to within better than chemical accuracy. Other methods, e.g. the MS interpolation method, ${ }^{482,483}$ the PLEPS ${ }^{494}$ and FPLEPS ${ }^{494,496}$ methods, and the POTFIT ${ }^{502-504}$ method have also been discussed.

The choice of the dynamical model is governed by the need to model one or both of the two dissipative channels in DC on metals, i.e., phonon excitation on the one hand and ehp excitation, or more generally electronic non-adiabaticity, on the other hand. ${ }^{266}$ This may be important for accurately extracting $E_{0}$ from simulations of $\mathrm{MB}$ experiments on DC on metals ${ }^{59}$ and for modeling the effect of the energy released in such reactions on the overall heterogeneously catalyzed process they may be part of. $^{266}$ The BOSS model usually allows a chemically accurate description of activated DC of dihydrogen on metals, ${ }^{28}$ but only yields semi-quantitative accuracy for DC of heavier diatomic and polyatomic molecules, for which the effect of surface atom motion needs to be modeled (BOMS). ${ }^{50-53}$ For quantitative 
accuracy it is usually necessary to model motion in all molecular DOFs, although in many cases this might be achievable by appropriately averaging over dynamics results obtained for a number of fixed impact sites (SAED). $61,76,102,106,621$

The BOMS model implementations discussed in Section 4.1.2 differ in (i) which couplings (electronic and/or mechanical) between molecular and phonon motion are taken into account, (ii) whether instantaneous couplings are modeled, (iii) the number of surface DOFs or the phonon fine structure modeled, (iv) computational expense, $(v)$ whether they are applied a posteriori, and (vi) whether energy dissipation away from the reaction zone is modeled (Table 4). A popular method used with QCT calculations is the GLO model; ${ }^{520,521}$ a recent extension is the MGLO model, ${ }^{153}$ which in addition models electronic coupling and improves the modeling of mechanical coupling. The LRS method of Jackson and co-workers ${ }^{524,525}$ is an accurate and popular, inexpensive method for modeling phonon effects in an a posteriori fashion, while taking both electronic and mechanical coupling into account. The DFMD method ${ }^{532}$ is expensive to use but accurate if the motion of the molecule and the surface atoms can be modeled quasi-classically; in this case one may also use the QCT method with a HDNNP ${ }^{133,139}$ to achieve higher statistical accuracy at reduced computational expense. Other model implementations discussed in Section 4 include the SO model, ${ }^{515,516}$ the SM model, ${ }^{516}$ the PSA, ${ }^{171}$ the SCM ${ }^{531}$ and the QM/Me embedding scheme. ${ }^{96}$

In the NBOSS model the BO approximation is abandoned but surface atom motion is neglected. In high-dimensional dynamics calculations the effect of ehp excitation is then modeled either through an EF method, ${ }^{54,57,58}$ assuming weak coupling, a surface hopping method (allowing changes in the electronic states of the molecule), ${ }^{574}$ or the IESH method (allowing both ehp excitation and other electronic state changes of the system). ${ }^{128}$ Using MDEF, ${ }^{551,558}$ the effect of ehp excitation has been modeled with the LDFA ${ }^{54}$ and the ODF model. ${ }^{57,551,558-560}$ The LDFA represents a rigorous theory for scattering of atoms, but it is approximate for molecules as it neglects molecular electronic structure effects. ${ }^{55,557}$ The ODF model $^{57,551,558-560}$ takes the electronic structure of the molecule and the metal into account but has a contradiction built into it $^{561}$ which can however be dealt with pragmatically. ${ }^{57,58}$ Effects of ehp excitation on DC of molecules on metals modeled with the LDFA have so far been found to be small, ${ }^{48,54,57-59,85,99,122,140,174,266,511}$ but non-adiabatic effects on DC on metals continue to be studied for fundamental reasons and due to their potential importance to heterogeneous catalysis. $^{266}$ Also, effects of ehp excitation can be considerably larger when studied with ODF, as shown for $\mathrm{N}_{2}+\mathrm{Ru}(0001)$ where modeling these effects halved computed $S_{0}$ in BOMS calculations. ${ }^{59}$ The IESH method has, to our knowledge, not yet been applied to DC on metals. Whether non-adiabatic spin transitions of $\mathrm{O}_{2}$ have a strong effect on its $\mathrm{DC}$ on $\mathrm{Al}(111)$, which has been studied on the basis of DFT with constraints and with a surface hopping method, remains an issue of high interest. ${ }^{399}$

Non-adiabatic effects and surface atom motions are both modeled with the NBOMS model. Model implementations include the GLO approach for surface phonons combined with a generalized Langevin treatment of ehp excitation modeled with the LDFA, ${ }^{69,109,143,146,168}$ MDEF with a HDNNP and the use of either the $\mathrm{LDFA}^{59,133,138}$ or $\mathrm{ODF}{ }^{59}$ and the $\mathrm{DFMDEF}^{74,114,147,168,582}$ method. In the last two approaches, the electron density associated with the mobile surface may be efficiently modeled with a Hirshfeld partitioning scheme. ${ }^{74,557}$ Concerning the effects of LDFA friction and of surface atom motion, studies ${ }^{264,266}$ have concluded that surface atom motion usually has larger effects on DC on metals than ehp excitation. DC is inhibited by ehp excitation when activated, but may be promoted by it when trapping mediated. ${ }^{264}$ Ehp excitation dominates energy dissipation of hyperthermal $\mathrm{H}$-atoms and of $\mathrm{H}$-atoms resulting from DC, and generally becomes the more important the lighter the atoms that are affected by dissipative forces are. ${ }^{264}$ Also, energy loss to ehp excitation is determined by the electron density in the regions the atoms (molecule) travel(s) through with high velocity. ${ }^{264}$

In the dynamics the equations of motion for the nuclei may often be solved quite accurately with the QCT method. This method will usually allow very accurate calculations of $S_{0}$ measured with MBs for activated DC of diatomic molecules, ${ }^{511}$ as for low $E_{i}$ the sticking tends to be dominated by the vibrationally excited molecules in the MB. ${ }^{186}$ In computing state-to-state scattering probabilities attention has to be paid to the binning method used to assign final states. ${ }^{133,168,174,594-596}$ Errors in results of QCT calculation may result from neglect of tunneling, zpe violation, and artificial IVR of vibrationally excited polyatomic molecules in the gas phase. Tunneling usually does not play a large role except perhaps at very low $E_{i}{ }^{51}$ Problems with artificial gas phase IVR may be kept small by considering partially deuterated isotopologues of the molecule of interest, and avoiding the simulation of experiments performed at high $T_{\mathrm{N}}{ }^{47}$ Recent calculations with the RPMD method suggest that zpe violation might well decrease the accuracy of calculations on DC of $\mathrm{D}_{2} \mathrm{O}$ on $\mathrm{Ni}(111),{ }^{597}$ but evidence exists that zpe violation effects on methane dissociation are small. $^{51}$

On the fly dynamics methods like DFMD are based on classical mechanics like the QCT method. An advantage of DFMD is that surface atom motion can be modeled while avoiding the need to fit a high-dimensional PES, but DFMD is computationally expensive.

QD calculations on DC of diatomic molecules have usually $^{28,106,508,509,591,592}$ (but not always ${ }^{507,510}$ ) been done with the TDWP method. It is important to treat all molecular DOFs ${ }^{508}$ although dynamical approximations to specific DOFs may sometimes work well. ${ }^{76,102,106}$ For DC of $\mathrm{H}_{2} \mathrm{O}$ on metals 9D TDWP calculations (i.e., treating all molecular DOFs) are now possible and have been performed on $\mathrm{H}_{2} \mathrm{O}+\mathrm{Cu}(111)^{61}$ and $\mathrm{H}_{2} \mathrm{O}$ $+\mathrm{Ni}(100) .{ }^{98}$ The only MB experiments on sticking of water to a metal surface have been performed for $\mathrm{D}_{2} \mathrm{O}+\mathrm{Ni}(111),{ }^{78}$ and approximate 9D TDWP calculations (i.e., 7D with the SAED approximation) have been performed on this system with 2 DFs, i.e., PW91 ${ }^{120}$ and RPBE. ${ }^{155}$ Averaging approximations involving only shifting of reaction probability curves computed for one impact site or azimuthal angle tend not to work for DC 
of $\mathrm{H}_{2} \mathrm{O} .{ }^{61,623}$ Instead, explicit dynamics calculations are required for different sites ${ }^{61,98,623}$ and azimuthal angles, ${ }^{61,100,120}$ and the sticking probabilities computed in this way then need to be averaged, so that the full topology of the PES and not just the barrier height is taken into account. The convergence of the 9D TDWP calculations with the scattering basis set size may require more testing in future.

Taking into account all molecular degrees of freedom in DC of $\mathrm{CH}_{4}$ on metals would require modeling motion in 15 DOFs. This is not yet possible with the TDWP method. The largest calculations have been performed for fixed impact sites and applying averaging over these sites, and the $C_{3 \mathrm{v}}$ symmetry of the remaining $\mathrm{CH}_{3}$ fragment is usually maintained, so that nine molecular DOFs are explicitly treated. ${ }^{123,145}$ Work on the gas phase $\mathrm{CH}_{4}+\mathrm{H}$ reaction suggests that applying the latter approximation may lead to errors in $S_{0}$ for the initial rovibrational ground state, and for laser-off reaction, of up to $50 \%{ }^{632}$ Errors in $S_{0}$ for initially vibrationally excited states may be even larger. ${ }^{632,633}$ Usually only one of the $\mathrm{CH}$ bonds is allowed to dissociate, and research groups differ in whether they therefore multiply the computed $S_{0}$ by a symmetry factor $4,{ }^{60}$ or leave out this multiplication. $^{73,123,145,456,528}$ Although the TDWP calculations obviously take into account quantum effects and effects of surface atom motion have been treated ${ }^{60,73,528}$ in an a posteriori fashion using the LRS model ${ }^{524,525}$ or its VTSR implementation, ${ }^{528}$ these calculations are probably less accurate for DC of methane on metals than are DFMD and QCT calculations using HDNNPs.

The great advantage of the RPH method ${ }^{50,203}$ is that it is a QD method that can explicitly model all molecular vibrational DOFs in DC of molecules like $\mathrm{H}_{2} \mathrm{O}, \mathrm{CH}_{4}$, and $\mathrm{CO}_{2}$. Disadvantages are that approximations need to be made to the rotations of the molecule, ${ }^{50,203}$ its motion parallel to the surface, ${ }^{50,203}$ and that rigorous convergence with respect to the vibrational basis set size is not completely certain. ${ }^{89}$ The effect of $T_{\mathrm{S}}$ can be rather accurately modeled ${ }^{21,30}$ with the LRS method. ${ }^{524,525}$ Guidelines are now available on how to best approximate the rotations (through a mix ${ }^{162,178,179,527}$ of the $\mathrm{RAA}^{50}$ and the $\mathrm{RSA}^{89}$ that depends on $E_{i}$ ) and how to best average over parallel translational motion. ${ }^{89,123} \mathrm{RPH}$ calculations on $\mathrm{CHD}_{3}+\mathrm{Pt}(111)$ were in good agreement with DFMD calculations using the same DF for conditions under which classical mechanics should be valid. ${ }^{51,527}$

Recently the NE-RPMD method has been tested on $\mathrm{H}_{2}+\mathrm{Cu}(111)$ and $\mathrm{D}_{2} \mathrm{O}+\mathrm{Ni}(111) .{ }^{597}$ First results suggest that this method may be used to study sticking under conditions where QD calculations would be required but are presently intractable. However, the results for $\mathrm{H}_{2}+\mathrm{Cu}(111)$ were inconclusive, and sticking has not yet been addressed for realistic MB conditions. Additional studies with this method are needed to establish its reliability.

Observables that can be computed with dynamics methods include $S_{0}\left(E_{i}^{\mathrm{av}} ; T_{\mathrm{N}}\right), R_{v j}\left(E_{i}\right), P_{v j \rightarrow v^{\prime} j^{\prime} m m}\left(E_{i}\right), P_{v j_{\mathrm{r}} \rightarrow v^{\prime} j_{\mathrm{r}}^{\prime}}\left(E_{i}\right), P_{v j_{\mathrm{r}} \rightarrow v^{\prime}}\left(E_{i}\right)$, $A_{0}^{(2)}(J)$, and ADEs. As discussed, the accurate calculation of these quantities puts demands on the dynamical model to be used that are specific to these quantities. For an optimal comparison of computed $S_{0}\left(E_{i}^{\text {av }} ; T_{\mathrm{N}}\right)$ with experimental values, it is best if the MB conditions of the measurements $\left(T_{\mathrm{N}}\right.$, stream velocity, and velocity width of the beam) are well documented, ${ }^{163}$ and this is especially true for activated DC, and even more so for activated DC of pure beams of $\mathrm{H}_{2}{ }^{43}$

SRP-DFs have now been derived for 7 systems. SRP-DFs for $\mathrm{H}_{2}+\mathrm{Cu}(111)$ include mixtures of GGA DFs, ${ }^{43,175}$ a combination of a GGA exchange DF with the vdW1 DF, ${ }^{150}$ combinations of GGA exchange DFs and vdW2 correlation, ${ }^{658}$ and mGGA DFs of the made-simple type. ${ }^{152}$ These SRP-DFs have allowed $S_{0}{ }^{43,175,658}$ and $E_{0}^{v j}$ measured for $\mathrm{H}_{2}{ }^{43,658}$ and $\mathrm{D}_{2},{ }^{175}$ and the ratio of $P(v=1, j=0 \rightarrow v=1, j=2)$ and $P(v=1, j=0 \rightarrow v=1, j=0)$ measured for $\mathrm{H}_{2}{ }^{43,658}$ to be reproduced with chemical accuracy. The optPBE-vdW ${ }^{331}$ SRP-DF found to accurately describe ${ }^{150} \mathrm{DC}$ of $\mathrm{H}_{2}$ on $\mathrm{Cu}(111)$ was used successfully to model the abstraction of $\mathrm{D}$ by $\mathrm{H}$ or $\mathrm{H}$ by $\mathrm{D}$ from $\mathrm{Cu}(111)$ resulting in $\mathrm{HD} .{ }^{583}$

The SRP43 DF developed for $\mathrm{H}_{2}$ on $\mathrm{Cu}(111)^{43}$ turned out to also be a SRP-DF for $\mathrm{H}_{2}+\mathrm{Cu}(100)$, for which it reproduced measured $S_{0}$, probabilities for rotationally inelastic scattering of $v=1 \mathrm{D}_{2}$, and measured $A_{0}^{(2)}(J)$ of $\mathrm{H}_{2}$ desorbing from $\mathrm{Cu}(100)$ in some, but not all of the $(v=0$ and $1, j)$ states for which measured values are available. The optPBE-vdW1 $\mathrm{DF}^{331}$ was found to be an SRP-DF for $\mathrm{H}_{2}+\mathrm{Cu}(111), \mathrm{Cu}(100)$, and $\mathrm{Cu}(110) \cdot{ }^{467}$

An SRP DF for $\mathrm{H}_{2}+\mathrm{Pt}(111),{ }^{156}$ which is a combination of a GGA exchange DF $\left(\mathrm{PBE}^{382}\right.$ with $\left.\alpha=0.57\right)$ and the vdW2 correlation $\mathrm{DF}{ }^{327}$ reproduces measured $S_{0}$ for both normal and off-normal incidence, where in the system addressed normal energy scaling is not observed. Calculations with the developed SRP-DF allowed an understanding of differences in the $S_{0}$ measured for $\mathrm{D}_{2}+\mathrm{Pt}(111)$ by three different groups. The B86SPR68-DF2 functional, which is an SRP-DF for $\mathrm{H}_{2}+\mathrm{Cu}(111)$, was also found to be an SRP-DF for $\mathrm{H}_{2}+\operatorname{Pt}(111) .{ }^{658}$ In turn, the PBE $\alpha 57-\mathrm{vdW} 2$ SRP DF for $\mathrm{H}_{2}+$ $\operatorname{Pt}(111)^{156}$ was also found to describe sticking of $\mathrm{H}_{2}$ and $\mathrm{D}_{2}$ on $\mathrm{Cu}(111)$ with chemical accuracy. ${ }^{658}$

The other three systems for which SRP-DFs were developed were all $\mathrm{CH}_{4}$-metal surface systems. ${ }^{44,47}$ All calculations establishing the SRP-DF were done for and fitted to experiments on $\mathrm{CHD}_{3}$ reacting on metals. ${ }^{44,47}$ With a correctly fitted SRP-DF good agreement could be obtained with laser-off experiments using $T_{\mathrm{N}} \leq$ $650 \mathrm{~K}^{44,47}$ The first $\mathrm{CH}_{4}$-metal system an SRP-DF was obtained for is $\mathrm{CHD}_{3}+\mathrm{Ni}(111)$, for which the fit was performed by comparison to laser-off sticking experiments, while initial-state-selected experiments on $\nu_{1}=1 \mathrm{CHD}_{3}$ were used for validation. ${ }^{44}$ In all cases DFMD calculations were used. The SRP32-vdW1 DF used was a 32/68 weighted average of the RPBE and PBE exchange DFs, in combination with vdW1 correlation. ${ }^{44}$ Using the SRP32-vdW1 DF, calculations with the RPH method ${ }^{673}$ gave a qualitatively correct description of experiments on vibrationally elastic and inelastic scattering of $\mathrm{CH}_{4}$ from Ni(111). ${ }^{240}$ A 15D PES for $\mathrm{CH}_{4}$ interacting with a static Ni(111) surface and computed with the SRP32-vdW1 DF is available. ${ }^{167}$ Interestingly, the SRP32-vdW1 DF also is a SRP-DF for $\mathrm{CHD}_{3}+\mathrm{Pt}(111)$ and $\mathrm{Pt}(211),{ }^{47}$ where reaction at both normal incidence $^{47}$ and with incidence perpendicular to the steps ${ }^{165}$ is correctly described with this DF. The potential transferability of SRP-DFs among systems in which the same molecule interacts with both low index and stepped surfaces of the same metal suggests a way to at least partially bridge the materials gap between surface science and heterogeneous catalysis. ${ }^{47}$ 
Candidate SRP-DFs have been developed for four additional systems. Both the PBE-vdW2 and the PBE:RPBE(50:50)-vdW1 DFs are c-SRP DFs for $\mathrm{H}_{2}+\mathrm{Ru}(0001)$, for which it was necessary to use either vdW1 or vdW2 correlation to achieve good agreement with sticking experiments. ${ }^{151}$ An attempt to validate the c-SRP DF through comparison with diffraction experiments failed. ${ }^{151}$ The former c-SRP $\mathrm{DF}$ for $\mathrm{H}_{2}+\mathrm{Ru}(0001)$ also is a c-SRP DF for $\mathrm{H}_{2}+\mathrm{Ni}(111) .{ }^{476}$ Furthermore, the SRP-DF for $\mathrm{H}_{2}+$ $\operatorname{Pt}(111)(\mathrm{PBE} \alpha=0.57-\mathrm{vdW} 2 \mathrm{DF})^{156}$ also turned out to be a c-SRP DF for $\mathrm{H}_{2}+\mathrm{Pt}(211){ }^{48}$ Finally, the RPBE DF is a c-SRP DF for $\mathrm{N}_{2}+\mathrm{Ru}(0001)$, for which both sticking and scattering experiments are well described with this DF. ${ }^{59}$ However, some uncertainty remains for this system, which is due to the error bars on the experimental $S_{0}$ being large and lack of systematic evidence concerning the accuracy of the LDFA and ODF methods for describing the effects of ehp excitation on sticking on and scattering from metal surfaces. ${ }^{59}$

There are still quite a few challenges to SRP-DFT as there are a number of systems for which SRP-DFs and cSRP-DFs have not yet been developed. Systems for which attempts have been made, or can be said to have been made that failed so far, include $\mathrm{H}_{2}$ interacting with $\mathrm{Ag}(111),{ }^{152,186,658} \mathrm{Pd}(111),{ }^{158} \mathrm{CO} /$ $\mathrm{Ru}(0001),{ }^{188} \mathrm{~N}_{2}$ interacting with $\mathrm{W}(100)^{132}$ and $\mathrm{W}(110),{ }^{88,101,149}$ $\mathrm{O}_{2}+\mathrm{Al}(111),{ }^{351,569,570} \mathrm{HCl}+\mathrm{Au}(111),{ }^{85,133,134,136,147,695} \mathrm{D}_{2} \mathrm{O}+$ $\mathrm{Ni}(111),{ }^{155} \mathrm{NH}_{3}+\mathrm{Ru}(0001),{ }^{66,535}$ and $\mathrm{CHD}_{3}$ interacting with $\operatorname{Pt}(210)^{181}$ and $\operatorname{Pt}(110)-(2 \times 1) .{ }^{182}$ These failed attempts pose challenges to the theory (for instance, the surface relaxation of $\operatorname{Pt}(110)-(2 \times 1)$ was not well described with the GGA-exchange based SRP-DF developed for $\mathrm{CHD}_{3}+\operatorname{Pt}(111)$ and $\left.\mathrm{Pt}(211)\right){ }^{182}$ but also to experiments. For example, the parameters of the molecular beams used in the experiments on $\mathrm{H}_{2}$ reacting on $\operatorname{Ag}(111)^{677}$ and $\operatorname{Pd}(111)^{678-682}$ were not well documented, and some of the experiments on $\mathrm{H}_{2}+\mathrm{Pd}(111)$ may have been performed at a too low $T_{\mathrm{s}}\left(223 \mathrm{~K},{ }^{681}\right.$ see Section 5.3.2). Also, no LEED-experiments have been done to ascertain an even coverage of the $\mathrm{Ru}(0001)$ surface by $\mathrm{CO}^{188}$ in the experiments on $\mathrm{H}_{2}$ sticking on CO-covered $\mathrm{Ru}(0001) .{ }^{685}$

Dynamics calculations based on SRP-DFs developed for related systems have resulted in predictions for $\mathrm{H}_{2}$ interacting with $\mathrm{Au}(111)^{187}$ and $\mathrm{Cu}(211),{ }^{166} \mathrm{HOD}+\mathrm{Ni}(111),{ }^{184} \mathrm{CHD}_{3}$ interacting with $\mathrm{Pd}(111),{ }^{533} \mathrm{Cu}(111),{ }^{63,185}$ single atom surface alloys of $\mathrm{Cu}(111),{ }^{185}$ and $\mathrm{Cu}(211),{ }^{185} \mathrm{CH}_{4}+\mathrm{Ni}(211),{ }^{178,179} \mathrm{CH}_{3} \mathrm{OH}+$ $\mathrm{Cu}(111),{ }^{183}$ and scattering of $\mathrm{H}$-atoms from $\mathrm{Au}(111)^{180,189-192}$ and $\mathrm{Cu}(111){ }^{189,191}$ The predictions for $\mathrm{H}_{2}+\mathrm{Au}(111)$ were later tested in associative desorption experiments, ${ }^{23}$ which confirmed some of the predictions, but not all of them. The experimentalists attributed discrepancies between some of the computed and measured ADEs to the neglect of ehp excitation in the calculations on this system. ${ }^{23}$ Very recent theoretical work using one of the MS mGGA DFs and DFs combining GGA exchange with vdW1 and vdW2 correlation did not yield improved agreement. ${ }^{658}$ Possible theoretical improvements for this system include modeling the effects of surface reconstruction, of $T_{\mathrm{s}}$, and of ehp excitation. ${ }^{658}$ The calculations on $\mathrm{H}_{2}+\mathrm{Cu}(211)$, which yielded $S_{0}$ in good agreement with experiments, ${ }^{166}$ showed that the steps do not increase $S_{0}$ relative to the flat $\mathrm{Cu}(111)$ surface, which was a surprising result. ${ }^{166}$ Results of associative desorption experiments on $\mathrm{H}_{2}, \mathrm{D}_{2}+\mathrm{Cu}(211)$ have now also been published ${ }^{194}$ and QCT calculations using the SRP48 DF were able to reproduce the $E_{1 / 2}^{v j}$ parameters measured for $\mathrm{H}_{2}+\mathrm{Cu}(111)$ to within chemical accuracy. ${ }^{511}$ However, theory has yet to find and explain the "slow" associative desorption channel" ${ }^{511}$ found recently for $\mathrm{H}_{2}+\mathrm{Cu}(111)$ and $\mathrm{Cu}(211) .{ }^{194}$

The predictive calculations on $\mathrm{CHD}_{3}$ interacting with $\mathrm{Cu}(111)$ suggested a very high vibrational efficacy of $\nu_{1}=2 \mathrm{CHD}_{3}$, due to the reaction of vibrational ground state and of $\nu_{1}=1 \mathrm{CHD}_{3}$ being hindered by the bobsled effect. ${ }^{63}$ Calculations using TST on $\mathrm{CH}_{4}$ reacting on a stepped $\mathrm{Ni}(111)$ surface with (111) terraces yield excellent agreement with experimental sticking rates at the (100) steps of these surfaces, ${ }^{178}$ suggesting that the SRP-DF developed for methane interacting with Ni(111), $\operatorname{Pt}(111)$, and $\mathrm{Pt}(211)$ indeed describes these systems with high accuracy even though classical mechanics was used to derive the SRP-DF.

In it self it is surprising that SRP-DFT based on GGA DFs (or on GGA exchange DFs combined with vdW1 or vdW2 correlation DFs) is able to model DC on metals accurately, given that GGADFT systematically underestimates gas phase reaction barrier heights. ${ }^{31,289}$ A clue for the reason behind this difference is given by the dependence of the success of GGA-based DFs for DC on metals on $(\Phi$-EA), which is high $(>7 \mathrm{eV})$ for systems for which SRP-DFT has been successful and low $(<7 \mathrm{eV})$ for systems for which it has not been successful yet. For the latter case it was not yet possible to obtain SRP-DFs or c-SRP DFs based on GGA exchange DFs, ${ }^{351}$ with the $\mathrm{O}_{2}+\mathrm{Al}(111)$ and $\mathrm{HCl}+$ $\mathrm{Au}(111)$ systems being notorious examples of this category. A (hand waving) explanation of this observation is that for $(\Phi$-EA $)>7 \mathrm{eV}$ error cancellation occurs between the electrons involved in the breaking and forming of new bonds in the DC, with the electrons coming from the molecule becoming more delocalized and the electrons coming from the metal becoming more localized in the TS. ${ }^{4,351}$ For $(\Phi$-EA $)<7 \mathrm{eV}$ electron transfer from the metal surface to the molecule occurs leading to increased overall electron delocalization in the TS, which GGA DFs "like", causing barriers to be too low, as also seen for gas phase reactions. ${ }^{351}$

Strategies for deriving SRP-DFs that have been discussed include starting from a suitable generic expression for the SRP-DF, brute force search aided by plots of the minimum barrier height vs. the energetic corrugation of the barrier, ${ }^{151,476}$ joint experimental-theoretical search strategies, ${ }^{44,47}$ and exploiting transferability of SRP-DFs for chemically related systems. ${ }^{46-48}$ The brute force search strategy can be justified on the basis of the hole model. ${ }^{45}$ The application of this strategy recognizes that the success of the SRP-DFT approach stems from the ability of semilocal density functionals to accurately describe how the barrier height for dissociative chemisorption depends on the moleculesurface configuration (as given by the impact site and molecular orientation for a diatomic molecule) $)^{151,177,383,429}$ and the tunability of the minimum barrier height, which for semi-local functions has been demonstrated for several systems obeying $(\Phi$-EA) $>7 \mathrm{eV}$.

Transferability of SRP-DFs among systems in which the same molecule interacts with low index and stepped surfaces 
of the same metal is potentially useful to modeling heterogeneous catalysis. ${ }^{164}$ Attempts to exploit transferability have not been universally successful; there are indications that SRP-DFs based on DFs from higher rungs of the DFT ladder may show higher transferability. ${ }^{152}$ In line with this observation, the generalpurpose revTPSS mGGA DF shows a better performance than the general purpose PBE mGGA DF for the two DC-on-metalsystems that both have been tested on. ${ }^{151,152}$

SRP-DFT for DC on metals still faces several daunting challenges. Perhaps the greatest challenge is extending the method to systems for which $(\Phi$-EA $)<7 \mathrm{eV}$, including the $\mathrm{O}_{2}+\mathrm{Al}(111)^{35,351}$ and $\mathrm{HCl}+$ $\mathrm{Au}(111)^{85,106,134,147,695}$ benchmark systems. In this context the only database existing so far for DC on metals (SBH10) ${ }^{49}$ may be biased in favoring the performance of GGA-DFT, as it only includes systems for which $(\Phi$-EA $)>7 \mathrm{eV}$. Other challenges include (i) the presence in the literature of insufficiently accurate or documented experiments ${ }^{59,149,158,186}$ on the DC and scattering of molecules on/from metal surfaces or the absence of additional experiments for validating c-SRP DFs, ${ }^{476}$ (ii) making accurate predictions for molecular diffraction while modeling the van der Waals interaction of molecules with metals, ${ }^{148,151,163}$ (iii) accurately modeling (a) vibrationally inelastic scattering in these systems, ${ }^{168,177}$ (b) the metal itself (crystal lattice constant and surface relaxation ${ }^{181,182}$ and thermal expansion $^{172,173}$ ), (c) trapping mediated reaction, ${ }^{590}$ (d) systems with deep molecular chemisorption wells in addition to DC barriers, ${ }^{143,149}$ and (e) quantum mechanical effects in polyatomic molecules scattering from metals. ${ }^{597}$ One should obviously use the correct dynamical model, i.e., also include the dissipative degrees of freedom that matter. ${ }^{175,192,264,517}$ Accurately modeling electronically non-adiabatic effects remains a formidable challenge, with questions being whether the LDFA or the ODF method better describes the effects of ehp excitation in MDEF, ${ }^{57,59}$ and for which systems friction methods are sufficiently accurate. ${ }^{724,725}$ Here a problem is that systems with low ( $\Phi$-EA) are both hard to study with GGADFT $^{351}$ and likely subject to electronic non-adiabaticity. ${ }^{268}$

We end with highlighting some of the successes of SRP-DFT. SRP-DFT has already been helpful with interpreting dynamical mechanisms and explaining trends for several reactive moleculemetal surface systems. ${ }^{44,47,51,165,166,168,175}$ Several predictions have been made for systems that are closely related to systems for which SRP-DFs have been derived, and these predictions can be tested by experiments. ${ }^{63,183-185,533}$ Perhaps most importantly, the SRP-DFs and c-SRP-DFs have already provided chemically accurate reaction barriers for 11 systems, ${ }^{43,44,46-48,59,151,156,467,476}$ some of which have been incorporated in the SBH10 database, ${ }^{49}$ while the others can be used to extend this database. These data and data that can be generated with SRP-DFT in future research can be and in one case have been used to validate electronic structure methods with a claim to high accuracy, like ECW theory $^{392,399}$ and DMC. ${ }^{34,427}$

\section{List of acronyms}

AA Azimuthal averaging approximation

ADE Average desorption energy
$\mathrm{BO}$

CRP

c-SRP DF

CT

CWF

CZPE

DC

DF

DFMD

DFMDEF

DFT

DFTB

DMC

DOF

DW

EA

ECW

EF

ehp

EMT

FPLEPS

FSA

GA

GGA

GLO

HDNN

HDNNP

$\mathrm{HF}$

IESH

$\mathrm{LCH}$

LD

LDA

LDFA

LRS

MD

$\mathrm{MB}$

$\mathrm{MCTDH}$

MDEF

mGGA

MGLO

MLFF

MRCI

MS

MSE

MUE

$\mathrm{nD}$

NE-RPMD

NGA

NL

NLD

NN

NNP
Born-Oppenheimer

Corrugation reducing procedure

Candidate specific reaction parameter functional Classical trajectory

Correlated wave function

Classical trajectory method with molecular zeropoint vibrational energy incorporated in the molecule-surface potential

Dissociative chemisorption

Density functional

Density functional theory molecular dynamics

Density functional theory molecular dynamics with electronic friction

Density functional theory

Density functional tight binding

Diffusion Monte-Carlo

Degree of freedom

Debye-Waller

Electron affinity

Embedded correlation wave function

Electronic friction

Electron-hole pair

Effective medium theory

Flexible periodic LEPS

Flat surface approximation

Gradient approximation

Generalized gradient approximation

Generalized Langevin oscillator

High-dimensional neural network

High-dimensional neural network potential

Hartree-Fock

Independent electron surface hopping method

Long range corrected range separated hybrid

Local dispersion

Local density approximation

Local density friction approximation

Lattice reconstruction sudden

Mean distance

Molecular beam

Multi-configuration time-dependent Hartree

Molecular dynamics with electronic friction

Meta-generalized gradient approximation

Modified generalized Langevin oscillator

Multilayer feed-forward

Multi-reference configuration interaction

Modified Shepard

Mean signed error

Mean unsigned error

$n$-Dimensional ( $n$ being a number, e.g. $6 \mathrm{D}=$ sixdimensional)

Non-equilibrium ring polymer molecular dynamics

Non-separable gradient approximation

Non-local

Non-local dispersion

Neural network

Neural network potential 


\begin{tabular}{|c|c|c|}
\hline ODF & Orbital-dependent friction & $J$ \\
\hline PES & Potential energy surface & \\
\hline PIP & Permutation invariant polynomial & $K$ \\
\hline PLEPS & Periodic LEPS & $M$ \\
\hline PSA & Phonon sudden approximation & \\
\hline QD & Quantum dynamics, quantum dynamical & $m_{j}$ \\
\hline QCT & Quasi-classical trajectory & $n$ \\
\hline QMC & Quantum Monte-Carlo & $N$ \\
\hline RAA & Rotationally adiabatic approximation & $N_{\mathrm{d}}$ \\
\hline RPA & Random phase approximation & $r$ \\
\hline RSA & Rotational sudden approximation & \\
\hline $\mathrm{RPH}$ & Reaction path Hamiltonian & \\
\hline RPMD & Ring polymer molecular dynamics & $r_{\mathrm{b}}$ \\
\hline RSH & Range-separated hybrid & $r_{1}$ \\
\hline SAED & Site-averaging of explicit dynamics results & \\
\hline SAEES & Site averaging by explicit energy shifting & $r_{2}$ \\
\hline SAHP & Site-averaging with harmonic potential & $\boldsymbol{R}$ \\
\hline SCM & Static corrugation model & $R_{v j}\left(E_{i}\right)$ \\
\hline SH & Screened hybrid & \\
\hline SI & Supporting Information & $Q$ \\
\hline SIE & Self-interaction error & \\
\hline SM & Surface mass & $Q$ \\
\hline SO & Surface oscillator & $s$ \\
\hline SRP & Specific reaction parameter & \\
\hline SRP-DF & Specific reaction parameter density functional & $S_{0}$ \\
\hline SRP-DFT & $\begin{array}{l}\text { Specific reaction parameter approach to density } \\
\text { functional theory }\end{array}$ & $T_{\mathrm{g}}$ \\
\hline TDDFT & Time-dependent density functional theory & $T_{\mathrm{N}}$ \\
\hline TDWP & Time-dependent wave packet & $T_{\text {rot }}$ \\
\hline $\mathrm{TM}$ & Transition metal & \\
\hline TOF & Time-of-flight & $T_{\mathrm{s}}$ \\
\hline TS & Transition state & $T_{\mathrm{vib}}$ \\
\hline TST & Transition state theory & \\
\hline TTEB & Total energy tight binding & $v$ \\
\hline vdW1 & vdW-DF1 functional & $v$ \\
\hline $\mathrm{vdW} 2$ & vdW-DF2 functional & $v_{0}$ \\
\hline WFT & Wave function theory & $\mathrm{x}$ \\
\hline $\mathrm{XC}$ & Exchange-correlation & $X, Y$ \\
\hline zpe & Zero-point vibrational energy & \\
\hline $6 \mathrm{D}$ & See $\mathrm{nD}$ & $\begin{array}{l}Z, Z_{\mathrm{CM}} \\
Z_{\mathrm{b}}\end{array}$ \\
\hline
\end{tabular}

\section{List of symbols}

$A_{0}^{(2)}(J)$
$E_{\mathrm{b}}$
$E_{\mathrm{b}}^{\mathrm{c}}$
$E_{i}$
$E_{i}^{\text {av }}$

$E_{\mathrm{t}}$
$j$
$j$
$j$

Rotational quadrupole alignment parameter (eqn (30))

(Minimum) reaction barrier height

Zero-point energy corrected barrier height

Incidence energy

Incidence energy averaged over flux-weighted velo-

city distribution of molecular beam (eqn (23))

Translational energy

Rotational state or quantum number

Vector of rotational quantum numbers without $M$ or $m_{j}$

Rotational angular momentum vector, or vector of rotational quantum numbers
Rotational quantum number of polyatomic molecule

Projection of $J$ on unique molecular axis

Magnetic rotational quantum number of polyatomic molecule (projection of $J$ on surface normal)

Magnetic rotational quantum number

Phonon state

Number of electrons

Number of nuclear degrees of freedom

Bond distance of diatomic molecule, or distance of centre-of-mass of $\mathrm{CH}_{4}$ to dissociating $\mathrm{H}$-atom

(Fig. 25)

Bond distance of dissociating bond at the barrier Bond distance of non-dissociative bond $\left(\mathrm{H}_{2} \mathrm{O}\right.$, Fig. 22)

Bond distance of dissociative bond $\left(\mathrm{H}_{2} \mathrm{O}\right.$, Fig. 22) Coordinates of the atoms in the incident molecule Initial state selected reaction probability at specific incidence energy (eqn (18) and (27))

Surface atom displacement coordinate perpendicular to surface

Vector of surface atom displacements

Bond length in remaining $\mathrm{CH}_{3}$ fragment in $\mathrm{CH}_{4}$ (Fig. 25)

Initial sticking coefficient, initial sticking probability (eqn (19))

Gas temperature

Nozzle temperature

Rotational temperature of the molecules in a molecular beam

Surface temperature

Vibrational temperature of the molecules in a molecular beam

Vibrational state or quantum number or velocity

Vector of vibrational quantum numbers

Stream velocity

Fraction of exact exchange

Co-ordinates for motion of molecule's centre-ofmass along surface

Distance of molecule's centre-of-mass to surface Distance of reaction barrier to the surface

Inhomogeneity parameter, or mixing parameter in SRP DF, or mechanical coupling parameter (eqn (5)), or azimuthal rotation angle of $\mathrm{H}_{2} \mathrm{O}$ (Fig. 22), or width of velocity distribution

Electronic coupling parameter (eqn (6))

Angle between non-dissociative $\mathrm{CH}$-bond and umbrella axis in inert $\mathrm{CH}_{3}$ fragment of $\mathrm{CH}_{4}$ (Fig. 25) Shifting energy

Angle of rotation about molecular axis (Fig. 22)

Work function

Electron localization function

Vibrational efficacy

Azimuthal rotation angle of $\mathrm{CH}_{4}$ (Fig. 25)

Rotation angle of $\mathrm{CH}_{4}$ about axis from $\mathrm{CH}_{4}$ centreof-mass to dissociative $\mathrm{H}$-atom (Fig. 25) 


$\begin{array}{ll}\varphi_{2} & \begin{array}{l}\text { Rotation angle of inert } \mathrm{CH}_{3} \text { fragment about its } \\ \text { umbrella axis in } \mathrm{CH}_{4} \text { (Fig. 25) }\end{array} \\ \theta_{i} & \begin{array}{l}\text { Angle of incidence with respect to surface normal } \\ \theta_{1}\end{array} \\ & \begin{array}{l}\text { Polar angle between dissociative and non-dissociative } \\ \text { bond in } \mathrm{H}_{2} \mathrm{O} \text {, (Fig. 22), or polar angle between Jacobi } \\ \text { coordinate } r \text { and surface normal of } \mathrm{CH}_{4} \text { (Fig. 25) }\end{array} \\ \theta_{2} & \text { Polar angle between dissociative bond and surface } \\ & \text { normal in } \mathrm{H}_{2} \mathrm{O} \text { (Fig. 22), or polar angle between } \\ & \text { Jacobi coordinate } r \text { of } \mathrm{CH}_{4} \text { and umbrella axis of } \\ & \text { remaining } \mathrm{CH}_{3} \text { (Fig. 25) } \\ \Theta_{\mathrm{D}} & \text { Surface Debye temperature } \\ \tau & \text { Kinetic energy density }\end{array}$

\section{Conflicts of interest}

There are no conflicts of interest to declare.

\section{Acknowledgements}

I am grateful for useful discussions with Bret Jackson, Bin Jiang, Hua Guo, Dong-Hui Zhang, Fabio Busnengo, Maite Alducin, Inaki Juaristi, Johannes Voss, Laurent Bonnet, Peter Saalfrank, Uwe Manthe, Mark Somers, Jörg Meyer, Daniel Auerbach, Rainer Beck, Arthur Utz, and Alec Wodtke. I am grateful for useful suggestions from Axel Groß, Jörg Behler, and Joost Bakker. Much of the work reported here would not have been possible without the contributions of former and present graduate students and post-docs and visiting graduate students, i.e., Cristina Díaz, Luca Sementa, Elham Nour Ghassemi, Francesco Nattino, Davide Migliorini, Helen Chadwick, Mark Wijzenbroek, Theophile Tchakoua, Paul Spiering, Nick Gerrits, and Egidius Smeets. Part of the work reported here was supported financially by the ERC (with the ERC-ADG-2013 grant No. 338580) and by NWO (through CW-TOP grants Nrs. 715.011.000 and 715.017.001) and through several grants of supercomputer time by NCF and NWO-EW.

\section{References}

1 R. Noyori, Nat. Chem., 2009, 1, 5-6.

2 C. A. Wolcott, A. J. Medford, F. Studt and C. T. Campbell, J. Catal., 2015, 330, 197-207.

3 M. K. Sabbe, M. F. Reyniers and K. Reuter, Catal. Sci. Technol., 2012, 2, 2010-2024.

4 I. Chorkendorff and J. W. Niemantsverdriet, Concepts of Modern Catalysis and Kinetics, Wiley-VCH Verlag GMBH \& Co., Weinheim, 2003.

5 G. Ertl, J. Vac. Sci. Technol., A, 1983, 1, 1247-1253.

6 K. Honkala, A. Hellman, I. N. Remediakis, A. Logadottir, A. Carlsson, S. Dahl, C. H. Cristensen and J. K. Nørskov, Science, 2005, 307, 555-558.

7 A. J. Medford, A. Vojvodic, J. S. Hummelshøj, J. Voss, F. Abild-Pedersen, F. Studt, T. Bligaard, A. Nilsson and J. K. Nørskov, J. Catal., 2015, 328, 36-42.
8 A. J. Medford, J. Wellendorff, A. Vojvodic, F. Studt, F. AbildPedersen, K. W. Jacobsen, T. Bligaard and J. K. Nørskov, Science, 2014, 345, 197-200.

9 C. Stegelmann, A. Andreasen and C. T. Campbell, J. Am. Chem. Soc., 2009, 131, 8077-8082.

10 G. J. Kroes, J. Phys. Chem. Lett., 2015, 6, 4106-4114.

11 J. K. Nørskov, F. Abild-Petersen, F. Studt and T. Bligaard, Proc. Natl. Acad. Sci. U. S. A., 2011, 108, 937-943.

12 H. A. Michelsen, C. T. Rettner, D. J. Auerbach and R. N. Zare, J. Chem. Phys., 1993, 98, 8294-8307.

13 L. Schröter, H. Zacharias and R. David, Phys. Rev. Lett., 1989, 62, 571-574.

14 R. R. Smith, D. R. Killelea, D. F. DelSesto and A. L. Utz, Science, 2004, 304, 992-995.

15 J. C. Polanyi, Science, 1987, 236, 680-690.

16 G. R. Darling and S. Holloway, Rep. Prog. Phys., 1995, 58, 1595-1672.

17 H. Hou, S. J. Gulding, C. T. Rettner, A. M. Wodtke and D. J. Auerbach, Science, 1997, 277, 80-82.

18 B. L. Yoder, R. Bisson and R. D. Beck, Science, 2010, 329, 553-556.

19 R. D. Beck, P. Maroni, D. C. Papageorgopoulos, T. T. Dang, M. P. Schmid and T. R. Rizzo, Science, 2003, 302, 98-100.

20 D. R. Killelea, V. L. Campbell, N. S. Shuman and A. L. Utz, Science, 2008, 319, 790-793.

21 V. L. Campbell, N. Chen, H. Guo, B. Jackson and A. L. Utz, J. Phys. Chem. A, 2015, 119, 12434-12441.

22 L. Diekhöner, L. Hornekaer, H. Mortensen, E. Jensen, A. Baurichter, V. V. Petrunin and A. C. Luntz, J. Chem. Phys., 2002, 117, 5018-5030.

23 Q. Shuai, S. Kaufmann, D. J. Auerbach, D. Schwarzer and A. M. Wodtke, J. Phys. Chem. Lett., 2017, 8, 1657-1663.

24 C. T. Rettner, D. J. Auerbach and H. A. Michelsen, Phys. Rev. Lett., 1992, 68, 2547-2550.

25 A. Hodgson, J. Moryl, P. Traversaro and H. Zhao, Nature, 1992, 356, 501-504.

26 D. Farías and R. Miranda, Prog. Surf. Sci., 2011, 86, 222-254.

27 A. Al Taleb, G. Anemone, L. Zhou, H. Guo and D. Farías, J. Phys. Chem. Lett., 2019, 10, 1574-1580.

28 G. J. Kroes and C. Díaz, Chem. Soc. Rev., 2016, 45, 3658-3700.

29 B. Jiang, M. H. Yang, D. Q. Xie and H. Guo, Chem. Soc. Rev., 2016, 45, 3621-3640.

30 H. Guo, A. Farjamnia and B. Jackson, J. Phys. Chem. Lett., 2016, 7, 4576-4584.

31 N. Mardirossian and M. Head-Gordon, Mol. Phys., 2017, 115, 2315-2372.

32 H. S. Yu, X. He and D. G. Truhlar, J. Chem. Theory Comput., 2016, 12, 1280-1293.

33 K. Duanmu and D. G. Truhlar, J. Chem. Theory Comput., 2017, 13, 835-842.

34 K. Doblhoff-Dier, J. Meyer, P. E. Hoggan and G. J. Kroes, J. Chem. Theory Comput., 2017, 13, 3208-3219.

35 R. R. Yin, Y. L. Zhang, F. Libisch, E. A. Carter, H. Guo and B. Jiang, J. Phys. Chem. Lett., 2018, 9, 3271-3277.

36 K. Raghavachari, G. W. Trucks, J. A. Pople and M. Headgordon, Chem. Phys. Lett., 1989, 157, 479-483. 
37 J. J. Zheng, Y. Zhao and D. G. Truhlar, J. Chem. Theory Comput., 2009, 5, 808-821.

38 Y. Zhao, N. Gónzalez-García and D. G. Truhlar, J. Phys. Chem. A, 2005, 109, 2012-2018.

39 S. Dahl, A. Logadottir, R. C. Egeberg, J. H. Larsen, I. Chorkendorff, E. Törnqvist and J. K. Nørskov, Phys. Rev. Lett., 1999, 83, 1814-1817.

40 T. Zambelli, J. Wintterlin, J. Trost and G. Ertl, Science, 1996, 273, 1688-1690.

41 S. J. Klippenstein, V. S. Pande and D. G. Truhlar, J. Am. Chem. Soc., 2014, 136, 528-546.

42 Y. Y. Chuang, M. L. Radhakrishnan, P. L. Fast, C. J. Cramer and D. G. Truhlar, J. Phys. Chem. A, 1999, 103, 4893-4909.

43 C. Díaz, E. Pijper, R. A. Olsen, H. F. Busnengo, D. J. Auerbach and G. J. Kroes, Science, 2009, 326, 832-834.

44 F. Nattino, D. Migliorini, G. J. Kroes, E. Dombrowski, E. A. High, D. R. Killelea and A. L. Utz, J. Phys. Chem. Lett., 2016, 7, 2402-2406.

45 M. Karikorpi, S. Holloway, N. Henriksen and J. K. Nørskov, Surf. Sci., 1987, 179, L41-L48.

46 L. Sementa, M. Wijzenbroek, B. J. van Kolck, M. F. Somers, A. Al-Halabi, H. F. Busnengo, R. A. Olsen, G. J. Kroes, M. Rutkowski, C. Thewes, N. F. Kleimeier and H. Zacharias, J. Chem. Phys., 2013, 138, 044708.

47 D. Migliorini, H. Chadwick, F. Nattino, A. GutiérrezGonzalez, E. Dombrowski, E. A. High, H. Guo, A. L. Utz, B. Jackson, R. D. Beck and G. J. Kroes, J. Phys. Chem. Lett., 2017, 8, 4177-4182.

48 E. N. Ghassemi, E. W. F. Smeets, M. F. Somers, G. J. Kroes, I. M. N. Groot, L. B. F. Juurlink and G. Füchsel, J. Phys. Chem. C, 2019, 123, 2973-2986.

49 S. M. Sharada, T. Bligaard, A. C. Luntz, G. J. Kroes and J. K. Nørskov, J. Phys. Chem. C, 2017, 121, 19807-19815.

50 B. Jackson and S. Nave, J. Chem. Phys., 2011, 135, 114701.

51 F. Nattino, H. Ueta, H. Chadwick, M. E. van Reijzen, R. D. Beck, B. Jackson, M. C. van Hemert and G. J. Kroes, J. Phys. Chem. Lett., 2014, 5, 1294-1299.

52 O. Galparsoro, R. Pétuya, J. I. Juaristi, C. Crespos, M. Alducin and P. Larrégaray, J. Phys. Chem. C, 2015, 119, 15434-15442.

53 X. J. Shen, A. Lozano, W. Dong, H. F. Busnengo and X. H. Yan, Phys. Rev. Lett., 2014, 112, 046101.

54 J. I. Juaristi, M. Alducin, R. Díez Muiño, H. F. Busnengo and A. Salin, Phys. Rev. Lett., 2008, 100, 116102.

55 A. C. Luntz, I. Makkonen, M. Persson, S. Holloway, D. M. Bird and M. S. Mizielinski, Phys. Rev. Lett., 2009, 102, 109601.

56 J. I. Juaristi, M. Alducin, R. Díez Muiño, H. F. Busnengo and A. Salin, Phys. Rev. Lett., 2009, 102, 109602.

57 P. Spiering and J. Meyer, J. Phys. Chem. Lett., 2018, 9, 1803-1808.

58 R. J. Maurer, B. Jiang, H. Guo and J. C. Tully, Phys. Rev. Lett., 2017, 118, 256001.

59 P. Spiering, K. Shakouri, J. Behler, G. J. Kroes and J. Meyer, J. Phys. Chem. Lett., 2019, 10, 2957-2962.

60 B. Jiang, R. Liu, J. Li, D. Q. Xie, M. H. Yang and H. Guo, Chem. Sci., 2013, 4, 3249-3254.
61 Z. J. Zhang, T. H. Liu, B. N. Fu, X. M. Yang and D. H. Zhang, Nat. Commun., 2016, 7, 11953.

62 Y. L. Zhang, X. Y. Zhou and B. Jiang, J. Phys. Chem. Lett., 2019, 10, 1185-1191.

63 N. Gerrits, K. Shakouri, J. Behler and G. J. Kroes, J. Phys. Chem. Lett., 2019, 10, 1763-1768.

64 Y. L. Zhang, R. J. Maurer, H. Guo and B. Jiang, Chem. Sci., 2019, 10, 1089-1097.

65 T. H. Liu, B. N. Fu and D. H. Zhang, J. Chem. Phys., 2018, 149, 174702.

66 X. X. Hu, M. Yang, D. Q. Xi and H. Guo, J. Chem. Phys., 2018, 149, 044703.

67 D. Ray, S. Ghosh and A. K. Tiwari, J. Phys. Chem. A, 2018, 122, 5698-5709.

68 S. Roy, S. Hariharan and A. K. Tiwari, J. Phys. Chem. C, 2018, 122, 10857-10870.

69 X. Luo, X. Y. Zhou and B. Jiang, J. Chem. Phys., 2018, 148, 174702.

70 X. Y. Zhou, B. Kolb, X. Luo, H. Guo and B. Jiang, J. Phys. Chem. C, 2017, 121, 5594-5602.

71 A. Farjamnia and B. Jackson, J. Chem. Phys., 2017, 146, 074704.

72 H. Seenivasan, B. Jackson and A. K. Tiwari, J. Chem. Phys, 2017, 146, 074705.

73 X. J. Shen, Z. J. Zhang and D. H. Zhang, J. Phys. Chem. C, 2016, 120, 20199-20205.

74 D. Novko, M. Blanco-Rey, M. Alducin and J. I. Juaristi, Phys. Rev. B, 2016, 93, 245435.

75 H. Guo and B. Jackson, J. Chem. Phys., 2015, 144, 184709.

76 X. X. Hu, B. Jiang, D. Q. Xie and H. Guo, J. Chem. Phys., 2015, 143, 114706.

77 A. Farjamnia and B. Jackson, J. Chem. Phys., 2015, 142, 234705.

78 P. M. Hundt, B. Jiang, M. E. van Reijzen, H. Guo and R. D. Beck, Science, 2014, 344, 504-507.

79 S. Nave, A. K. Tiwari and B. Jackson, J. Phys. Chem. A, 2014, 118, 9615-9631.

80 B. Jiang and H. Guo, Phys. Chem. Chem. Phys., 2014, 16, 24704-24715.

81 B. Jackson and S. Nave, J. Chem. Phys., 2013, 138, 174705.

82 I. Goikoetxea, J. Beltrán, J. Meyer, J. I. Juaristi, M. Alducin and K. Reuter, New J. Phys., 2012, 14, 013050.

83 M. Ramos, A. E. Martínez and H. F. Busnengo, Phys. Chem. Chem. Phys., 2012, 14, 303-310.

84 H. Chadwick, D. Migliorini and G. J. Kroes, J. Chem. Phys., 2018, 149, 044701.

85 G. Füchsel, M. del Cueto, C. Díaz and G. J. Kroes, J. Phys. Chem. C, 2016, 120, 25760-25779.

86 F. Nattino, A. Galparsoro, F. Costanzo, R. Diéz Muiño, M. Alducin and G. J. Kroes, J. Chem. Phys., 2016, 144, 244708.

87 G. Füchsel, P. S. Thomas, J. den Uyl, Y. Öztürk, F. Nattino, H.-D. Meyer and G. J. Kroes, Phys. Chem. Chem. Phys., 2016, 13, 8659-8670.

88 F. Nattino, F. Costanzo and G. J. Kroes, J. Chem. Phys., 2015, 142, 104702. 
89 B. Jackson, F. Nattino and G. J. Kroes, J. Chem. Phys., 2014, 141, 054102.

90 D. W. Han, S. Nave and B. Jackson, J. Phys. Chem. A, 2013, 117, 8651-8659.

91 X. X. Hu, Y. P. Zhou, B. Jiang, H. Guo and D. Q. Xie, Phys. Chem. Chem. Phys., 2017, 19, 12826-12837.

92 B. Jiang and H. Guo, J. Chem. Phys., 2016, 144, 091101.

93 A. Groß, Catal. Today, 2016, 260, 60-65.

94 I. Goikoetxea, J. Meyer, J. I. Juaristi, M. Alducin and K. Reuter, Phys. Rev. Lett., 2014, 112, 156101.

95 M. Grotemeyer and E. Pehlke, Phys. Rev. Lett., 2014, 112, 043201.

96 J. Meyer and K. Reuter, Angew. Chem., Int. Ed., 2014, 53, 4721-4724.

97 J. Meyer and K. Reuter, New J. Phys., 2011, 13, 085010.

98 T. H. Liu, J. Chen, Z. J. Zhang, X. J. Shen, B. N. Fu and D. H. Zhang, J. Chem. Phys., 2018, 148, 144705.

99 X. Luo, B. Jiang, J. I. Juaristi, M. Alducin and H. Guo, J. Chem. Phys., 2016, 145, 044704.

100 T. H. Liu, Z. J. Zhang, B. N. Fu, X. M. Yang and D. H. Zhang, Chem. Sci., 2016, 7, 1840-1845.

101 L. Martin-Gondre, J. I. Juaristi, M. Blanco-Rey, R. Díez Muiño and M. Alducin, J. Chem. Phys., 2015, 142, 074704.

102 T. H. Liu, B. N. Fu and D. H. Zhang, J. Chem. Phys., 2014, 141, 194302.

103 T. H. Liu, Z. J. Zhang, J. Chen, B. N. Fu and D. H. Zhang, Phys. Chem. Chem. Phys., 2016, 18, 26358-26364.

104 B. Jiang, X. X. Hu, S. Lin, D. Q. Xie and H. Guo, Phys. Chem. Chem. Phys., 2015, 17, 23346-23355.

105 T. H. Liu, B. N. Fu and D. H. Zhang, J. Chem. Phys., 2014, 140, 144701.

106 T. H. Liu, B. N. Fu and D. H. Zhang, J. Chem. Phys., 2013, 139, 184705.

107 Q. Fu and Y. Luo, J. Phys. Chem. C, 2013, 117, 14618-14624.

108 B. Jiang, J. Li, D. Q. Xie and H. Guo, J. Chem. Phys., 2013, 138, 044704.

109 L. Martin-Gondre, M. Alducin, G. A. Bocan, R. Díez Muiño and J. I. Juaristi, Phys. Rev. Lett., 2012, 108, 096101.

110 K. R. Geethalakshmi, J. I. Juaristi, R. Díez Muiño and M. Alducin, Phys. Chem. Chem. Phys., 2011, 13, 4357-4364.

111 M. N. Batista, H. F. Busnengo and A. E. Martinez, Phys. Chem. Chem. Phys., 2011, 13, 4614-4624.

112 G. Laurent, D. Barredo, D. Farías, R. Miranda, C. Díaz, P. Rivière, M. F. Somers and F. Martín, Phys. Chem. Chem. Phys., 2010, 12, 14501-14507.

113 D. Stradi, C. Díaz and F. Martín, Surf. Sci., 2010, 604, 2031-2035.

114 M. Blanco-Rey, J. I. Juaristi, R. Díez Muiño, H. F. Busnengo, G. J. Kroes and M. Alducin, Phys. Rev. Lett., 2014, 112, 103203.

115 C. Díaz, F. Martín, G. J. Kroes, M. Minniti, D. Farías and R. Miranda, J. Phys. Chem. C, 2012, 116, 13671-13678.

116 P. Nieto, D. Farías, R. Miranda, M. Luppi, E. J. Baerends, M. F. Somers, M. J. T. C. van der Niet, R. A. Olsen and G. J. Kroes, Phys. Chem. Chem. Phys., 2011, 13, 8583-8597.

117 C. Díaz, R. A. Olsen, H. F. Busnengo and G. J. Kroes, J. Phys. Chem. C, 2010, 114, 11192-11201.
118 A. Lozano, A. Groß and H. F. Busnengo, Phys. Rev. B: Condens. Matter Mater. Phys., 2010, 81, 121402.

119 B. Jiang, X. F. Ren, D. Q. Xie and H. Guo, Proc. Natl. Acad. Sci. U. S. A., 2012, 109, 10224-10227.

120 B. Jiang, H. W. Song, M. H. Yang and H. Guo, J. Chem. Phys., 2016, 144, 164706.

121 B. Jiang and H. Guo, Phys. Rev. Lett., 2015, 114, 166101.

122 B. Jiang, M. Alducin and H. Guo, J. Phys. Chem. Lett., 2016, 7, 327-331.

123 X. J. Shen, Z. J. Zhang and D. H. Zhang, J. Chem. Phys., 2016, 144, 101101.

124 I. Goikoetxea, M. Alducin, R. Diéz Muiño and J. I. Juaristi, Phys. Chem. Chem. Phys., 2012, 14, 7471-7480.

125 K. Golibrzuch, P. R. Shirhatti, I. Rahinov, A. Kandratsenka, D. J. Auerbach, A. M. Wodtke and C. Bartels, J. Chem. Phys., 2014, 140, 044701.

126 K. Golibrzuch, A. Kandratsenka, I. Rahinov, R. Cooper, D. J. Auerbach, A. M. Wodtke and C. Bartels, J. Phys. Chem. A, 2013, 117, 7091-7101.

127 R. Cooper, C. Bartels, A. Kandratsenka, I. Rahinov, N. Shenvi, K. Golibrzuch, Z. S. Li, D. J. Auerbach, J. C. Tully and A. M. Wodtke, Angew. Chem., Int. Ed., 2012, 51, 4954-4958.

128 N. Shenvi, S. Roy and J. C. Tully, Science, 2009, 326, 829-832.

129 S. Monturet and P. Saalfrank, Phys. Rev. B: Condens. Matter Mater. Phys., 2010, 82, 075404.

130 M. Ramos, C. Díaz, A. E. Martínez, F. Martín and H. F. Busnengo, J. Phys. Chem. C, 2018, 122, 15529-15538.

131 B. Jiang, Chem. Sci., 2017, 8, 6662-6669.

132 A. Pena-Torres, H. F. Busnengo, J. I. Juaristi, P. Larregaray and C. Crespos, Phys. Chem. Chem. Phys., 2018, 20, 19326-19331.

133 Q. H. Liu, X. Y. Zhou, L. S. Zhou, Y. L. Zhang, X. Luo, J. Guo and B. Jiang, J. Phys. Chem. C, 2018, 122, 1761-1769.

134 T. H. Liu, B. N. Fu and D. H. Zhang, J. Chem. Phys., 2017, 140, 164706.

135 M. Ramos, C. Díaz, A. E. Martínez, H. F. Busnengo and F. Martín, Phys. Chem. Chem. Phys., 2017, 19, 10217-10221.

136 B. Kolb and H. Guo, J. Chem. Phys., 2016, 145, 011102.

137 B. Jiang and H. Guo, J. Phys. Chem. C, 2016, 120, 8220-8226.

138 K. Shakouri, J. Behler, J. Meyer and G. J. Kroes, J. Phys. Chem. C, 2018, 122, 23470-23480.

139 K. Shakouri, J. Behler, J. Meyer and G. J. Kroes, J. Phys. Chem. Lett., 2017, 8, 2131-2136.

140 G. Füchsel, S. Schimka and P. Saalfrank, J. Phys. Chem. A, 2013, 117, 8761-8769.

141 I. Goikoetxea, J. I. Juaristi, R. Diéz Muiño and M. Alducin, Phys. Rev. Lett., 2014, 113, 066103.

142 I. Loncaric, G. Füchsel, J. I. Juaristi and P. Saalfrank, Phys. Rev. Lett., 2017, 119, 146101.

143 A. Pena-Torres, H. F. Busnengo, J. I. Juaristi, P. Larregaray and C. Crespos, J. Phys. Chem. C, 2019, 123, 2900-2910.

144 J. L. Chen, X. Y. Zhou, Y. L. Zhang and B. Jiang, Nat. Commun., 2018, 9, 4039.

145 X. J. Shen, Z. J. Zhang and D. H. Zhang, J. Chem. Phys., 2017, 147, 024702.

146 C. Ibarguen, P. Larregaray, A. Pena-Torres and C. Crespos, J. Phys. Chem. C, 2018, 122, 28856-28861. 
147 G. Füchsel, X. Y. Zhou, B. Jiang, J. I. Juaristi, M. Alducin, H. Guo and G. J. Kroes, J. Phys. Chem. C, 2019, 123, 2287-2299.

148 G. J. Kroes, M. Wijzenbroek and J. R. Manson, J. Chem. Phys., 2017, 147, 244705.

149 D. Migliorini, F. Nattino and G. J. Kroes, J. Chem. Phys., 2016, 144, 084702.

150 M. Wijzenbroek, D. M. Klein, B. Smits, M. F. Somers and G. J. Kroes, J. Phys. Chem. A, 2015, 119, 12146-12158.

151 M. Wijzenbroek and G. J. Kroes, J. Chem. Phys., 2014, 140, 084702.

152 E. W. F. Smeets, J. Voss and G. J. Kroes, J. Phys. Chem. A, 2019, 123, 5395-5406.

153 X. J. Zhou and B. Jiang, J. Chem. Phys., 2019, 150, 024704.

154 R. Cooper, I. Rahinov, Z. Li, D. Matsiev, D. J. Auerbach and A. M. Wodtke, Chem. Sci., 2010, 1, 55-61.

155 B. Jiang and H. Guo, Phys. Chem. Chem. Phys., 2016, 18, 21817-21824.

156 E. N. Ghassemi, M. Wijzenbroek, M. F. Somers and G. J. Kroes, Chem. Phys. Lett., 2017, 683, 329-335.

157 F. Nattino, D. Migliorini, M. Bonfanti and G. J. Kroes, J. Chem. Phys., 2016, 144, 044702.

158 J. M. Boereboom, M. Wijzenbroek, M. F. Somers and G. J. Kroes, J. Chem. Phys., 2013, 139, 244707.

159 C. Díaz, R. A. Olsen, D. J. Auerbach and G. J. Kroes, Phys. Chem. Chem. Phys., 2010, 12, 6499-6519.

160 P. Spiering, M. Wijzenbroek and M. F. Somers, J. Chem. Phys., 2018, 149, 234702.

161 X. Y. Zhou and B. Jiang, Sci. China: Chem., 2018, 61, 1134-1142.

162 H. Chadwick, H. Guo, A. Gutiérrez-González, J. P. Menzel, B. Jackson and R. D. Beck, J. Chem. Phys., 2018, 148, 014701.

163 E. N. Ghassemi, M. F. Somers and G. J. Kroes, J. Phys. Chem. C, 2019, 123, 10406-10418.

164 D. Migliorini, H. Chadwick and G. J. Kroes, J. Chem. Phys., 2018, 149, 094701.

165 H. Chadwick, A. Gutiérrez-González, D. Migliorini, R. D. Beck and G. J. Kroes, J. Phys. Chem. C, 2018, 122, 19652-19660.

166 G. Füchsel, K. Cao, S. Er, E. W. F. Smeets, A. W. Kleyn, L. B. F. Juurlink and G. J. Kroes, J. Phys. Chem. Lett., 2018, 9, 170-175.

167 X. Y. Zhou, F. Nattino, Y. L. Zhang, J. Chen, G. J. Kroes, H. Guo and B. Jiang, Phys. Chem. Chem. Phys., 2017, 19, 30540-30550.

168 G. J. Kroes, J. I. Juaristi and M. Alducin, J. Phys. Chem. C, 2017, 121, 13617-13633.

169 O. Godsi, G. Corem, Y. Alkoby, J. T. Cantin, R. V. Krems, M. F. Somers, J. Meyer, G. J. Kroes, T. Maniv and G. Alexandrowicz, Nat. Commun., 2017, 8, 15357.

170 F. Nattino, A. Genova, M. Guijt, A. S. Muzas, C. Díaz, D. J. Auerbach and G. J. Kroes, J. Chem. Phys., 2014, 141, 124705.

171 M. Bonfanti, M. F. Somers, C. Díaz, H. F. Busnengo and G. J. Kroes, Z. Phys. Chem., 2013, 227, 1397-1420.

172 A. Mondal, M. Wijzenbroek, M. Bonfanti, C. Díaz and G. J. Kroes, J. Phys. Chem. A, 2013, 117, 8770-8781.

173 A. Marashdeh, S. Casole, L. Sementa, H. Zacharias and G. J. Kroes, J. Phys. Chem. C, 2013, 117, 8851-8863.
174 A. S. Muzas, J. I. Juaristi, M. Alducin, R. Diéz Muiño, G. J. Kroes and C. Díaz, J. Chem. Phys., 2012, 137, 064707.

175 F. Nattino, C. Díaz, B. Jackson and G. J. Kroes, Phys. Rev. Lett., 2012, 108, 236104.

176 P. S. Thomas, M. F. Somers, A. W. Hoekstra and G. J. Kroes, Phys. Chem. Chem. Phys., 2012, 14, 8628-8643.

177 G. J. Kroes, C. Díaz, E. Pijper, R. A. Olsen and D. J. Auerbach, Proc. Natl. Acad. Sci. U. S. A., 2010, 107, 20881-20886.

178 H. Guo and B. Jackson, J. Chem. Phys., 2019, 150, 204703.

179 H. Guo, J. P. Menzel and B. Jackson, J. Chem. Phys., 2018, 149, 244704.

180 S. M. Janke, D. J. Auerbach, A. M. Wodtke and A. Kandratsenka, J. Chem. Phys., 2015, 143, 124708.

181 H. Chadwick, A. Gutiérrez-González, R. D. Beck and G. J. Kroes, J. Phys. Chem. C, 2019, 123, 14530-14539.

182 H. Chadwick, A. Gutiérrez-González, R. D. Beck and G. J. Kroes, J. Chem. Phys., 2019, 150, 124702.

183 N. Gerrits and G. J. Kroes, J. Chem. Phys., 2019, 150, 024706.

184 D. Migliorini, F. Nattino, A. K. Tiwari and G. J. Kroes, J. Chem. Phys., 2018, 149, 244706.

185 N. Gerrits, D. Migliorini and G. J. Kroes, J. Chem. Phys., 2018, 149, 224701.

186 E. N. Ghassemi, M. Somers and G. J. Kroes, J. Phys. Chem. C, 2018, 122, 22939-22952.

187 M. Wijzenbroek, D. Helstone, J. Meyer and G. J. Kroes, J. Chem. Phys., 2016, 145, 144701.

188 M. Wijzenbroek and G. J. Kroes, Phys. Chem. Chem. Phys., 2016, 18, 21190-21201.

189 G. J. Kroes, M. Pavanello, M. Blanco-Rey, M. Alducin and D. J. Auerbach, J. Chem. Phys., 2014, 141, 054705.

190 S. M. Janke, M. Pavanello, G. J. Kroes, D. Auerbach, A. M. Wodtke and A. Kandratsenka, Z. Phys. Chem., 2013, 227, 1467-1490.

191 M. Pavanello, D. J. Auerbach, A. M. Wodtke, M. Blanco-Rey, M. Alducin and G. J. Kroes, J. Phys. Chem. Lett., 2013, 4, 3735-3740.

192 O. Bünermann, H. Y. Jiang, Y. Dorenkamp, A. Kandratsenka, S. M. Janke, D. J. Auerbach and A. M. Wodtke, Science, 2015, 350, 1346-1349.

193 K. Cao, R. van Lent, A. W. Kleyn and L. B. F. Juurlink, Chem. Phys. Lett., 2018, 706, 680-683.

194 S. Kaufmann, Q. Shuai, D. J. Auerbach, D. Schwarzer and A. M. Wodtke, J. Chem. Phys., 2018, 148, 194703.

195 J. P. Perdew, K. Burke and M. Ernzerhof, Phys. Rev. Lett., 1996, 77, 3865-3868.

196 B. Hammer, L. B. Hansen and J. K. Nørskov, Phys. Rev. B: Condens. Matter Mater. Phys., 1999, 59, 7413-7421.

197 Z. G. Wu and R. E. Cohen, Phys. Rev. B: Condens. Matter Mater. Phys., 2006, 73, 235116.

198 P. Haas, F. Tran, P. Blaha and K. Schwarz, Phys. Rev. B: Condens. Matter Mater. Phys., 2011, 83, 205117.

199 J. P. Perdew, A. Ruzsinszky, G. I. Csonka, L. A. Constantin and J. W. Sun, Phys. Rev. Lett., 2009, 103, 026403.

200 I. M. N. Groot, H. Ueta, M. J. T. C. van der Niet, A. W. Kleyn and L. B. F. Juurlink, J. Chem. Phys., 2007, 127, 244701. 
201 L. B. F. Juurlink, P. R. McCabe, R. R. Smith, C. L. DiCologero and A. L. Utz, Phys. Rev. Lett., 1999, 83, 868-871.

202 M. P. Schmid, P. Maroni, R. D. Beck and T. R. Rizzo, J. Chem. Phys., 2002, 117, 8603.

203 S. Nave and B. Jackson, Phys. Rev. B: Condens. Matter Mater. Phys., 2010, 81, 233408.

204 M. P. D’Evelyn, A. V. Hamza, G. E. Gdoswki and R. J. Madix, Surf. Sci., 1986, 167, 451-473.

205 R. van Lent, S. V. Auras, K. Cao, A. J. Walsh, M. A. Gleeson and L. B. F. Juurlink, Science, 2019, 363, 155-157.

206 S. V. Auras, R. van Lent, D. Bashlakov, J. M. Piñeiros Bastidas, T. Roorda, R. Spierenburg and L. B. F. Juurlink, Angew. Chem., Int. Ed., 2020, 59, 2-9.

207 J. T. Cantin, G. Alexandrowicz and R. V. Krems, Phys. Rev. A, 2020, 101, 062703.

208 Y. Alkoby, H. Chadwick, O. Godsi, H. Labiad, M. Bergin, J. T. Cantin, I. Litvin, T. Maniv and G. Alexandrowicz, Nat. Commun., 2020, 11, 3110.

209 J. Werdecker, P. R. Shirhatti, K. Golibrzuch, C. Bartels, A. M. Wodtke and D. J. Harding, J. Phys. Chem. C, 2015, 119, 14722-14727.

210 L. Diekhöner, H. Mortensen, A. Baurichter, E. Jensen, V. V. Petrunin and A. C. Luntz, J. Chem. Phys., 2001, 115, 9028-9035.

211 H. E. Pfnür, C. T. Rettner, J. Lee, R. J. Madix and D. J. Auerbach, J. Chem. Phys., 1986, 85, 7452-7466.

212 C. T. Rettner, E. K. Schweizer and H. Stein, J. Chem. Phys., 1990, 93, 1442-1454.

213 M. Beutl, K. D. Rendulic and G. R. Castro, Surf. Sci., 1997, 385, 97-106.

214 M. Kurahashi and Y. Yamauchi, Phys. Rev. Lett., 2013, 110, 246102.

215 M. Kurahashi and Y. Yamauchi, Phys. Rev. Lett., 2015, 114, 016101.

216 M. Minniti, D. Farías, P. Perna and R. Miranda, J. Chem. Phys., 2012, 137, 074706.

217 L. Österlund, I. Zoric and B. Kasemo, Phys. Rev. B: Condens. Matter Mater. Phys., 1997, 55, 15452-15455.

218 A. Raukema, D. A. Butler, F. M. A. Box and A. W. Kleyn, Surf. Sci., 1996, 347, 151-168.

219 P. R. Shirhatti, J. Geweke, C. Steinsiek, C. Bartels, I. Rahinov, D. J. Auerbach and A. M. Wodtke, J. Phys. Chem. Lett., 2016, 7, 1346-1350.

220 J. Geweke, P. R. Shirhatti, I. Rahinov, C. Bartels and A. M. Wodtke, J. Chem. Phys., 2016, 145, 054709.

221 J. Geweke and A. M. Wodtke, J. Chem. Phys., 2020, 153, 164703.

222 D. P. Engelhart, R. J. V. Wagner, P. C. Johnsen, A. M. Wodtke and T. Schafer, Phys. Chem. Chem. Phys., 2015, 17, 11540-11545.

223 R. J. V. Wagner, N. Henning, B. C. Krüger, G. B. Park, J. Altschaffel, A. Kandratsenka, A. M. Wodtke and T. Schafer, J. Phys. Chem. Lett., 2017, 8, 4887-4892.

224 P. R. Shirhatti, I. Rahinov, K. Golibrzuch, J. Werdecker, J. Geweke, J. Altschaffel, S. Kumar, D. J. Auerbach, C. Bartels and A. M. Wodtke, Nat. Chem., 2018, 10, 592-598.
225 R. J. V. Wagner, B. C. Krüger, G. B. Park, M. Wallrabe, A. M. Wodtke and T. Schafer, Phys. Chem. Chem. Phys., 2019, 21, 1650-1655.

226 J. Neugebohren, D. Borodin, H. W. Hahn, J. Altschäffel, A. Kandratsenka, D. J. Auerbach, C. T. Campbell, D. Schwarzer, D. J. Harding, A. M. Wodtke and T. N. Kitsopoulos, Nature, 2018, 558, 280-283.

227 D. Borodin, I. Rahinow, P. R. Shirhatti, M. Huang, A. Kandratsenka, D. J. Auerbach, T. L. Zhong, H. Guo, D. Schwarzer, T. N. Kitsopoulos and A. M. Wodtke, Science, 2020, 369, 1461-1465.

228 I. Loncaric, M. Alducin, J. I. Juaristi and D. Novko, J. Phys. Chem. Lett., 2019, 10, 1043-1047.

229 M. Huang, X. Y. Zhou, Y. L. Zhang, L. S. Zhou, M. Alducin, B. Jiang and H. Guo, Phys. Rev. B, 2019, 100, 201407.

230 J. D. White, J. Chen, D. Matsiev, D. J. Auerbach and A. M. Wodtke, Nature, 2005, 433, 503-505.

231 N. H. Nahler, J. D. White, J. Larue, D. J. Auerbach and A. M. Wodtke, Science, 2008, 321, 1191-1194.

232 C. Steinsiek, P. R. Shirhatti, J. Geweke, C. Bartels and A. M. Wodtke, J. Phys. Chem. C, 2018, 122, 10027-10033.

233 B. C. Krüger, N. Bartels, A. M. Wodtke and T. Schafer, Phys. Chem. Chem. Phys., 2016, 18, 14976-14979.

234 B. C. Krüger, S. Meyer, A. Kandratsenka, A. M. Wodtke and T. Schäfer, J. Phys. Chem. Lett., 2016, 7, 441-446.

235 H. Chadwick, A. Gutiérrez-González and R. D. Beck, J. Chem. Phys., 2016, 145, 174707.

236 A. Gutiérrez-González, F. F. Crim and R. D. Beck, J. Chem. Phys., 2018, 149, 074701.

237 P. M. Hundt, M. E. van Reijzen, R. D. Beck, H. Guo and B. Jackson, J. Chem. Phys., 2017, 146, 054701.

238 P. M. Hundt, H. Ueta, M. E. van Reijzen, B. Jiang, H. Guo and R. D. Beck, J. Phys. Chem. A, 2015, 119, 12442-12448.

239 E. Dombrowski, E. Peterson, D. Del Sesto and A. L. Utz, Catal. Today, 2015, 244, 10-18.

240 J. Werdecker, M. E. van Reijzen, B. J. Chen and R. D. Beck, Phys. Rev. Lett., 2018, 120, 053402.

241 L. Chen, H. Ueta, H. Chadwick and R. D. Beck, J. Phys. Chem. C, 2015, 119, 14499-14505.

242 C. Papp, P. Tränkenschuh, R. Streber, T. Fuhrman, R. Denecke and H.-P. Steinrück, J. Phys. Chem. C, 2007, 111, 2177-2184.

243 A. Gutiérrez-González and R. D. Beck, Phys. Chem. Chem. Phys., 2020, 22, 17448-17459.

244 M. E. Torio and H. F. Busnengo, J. Phys. Chem. C, 2020, 124, 19649-19654.

245 H. Mortensen, L. Diekhöner, A. Baurichter, E. Jensen and A. C. Luntz, J. Chem. Phys., 2000, 113, 6882-6887.

246 L. Diekhöner, D. A. Butler, A. Baurichter and A. C. Luntz, Surf. Sci., 1998, 409, 384-391.

247 J. F. Weaver, M. A. Krzyzowski and R. J. Madix, J. Chem. Phys., 2000, 110, 396-407.

248 M. C. McMaster and R. J. Madix, Surf. Sci., 1993, 294, 420-428.

249 M. C. McMaster and R. J. Madix, J. Chem. Phys., 1993, 98, 9963-9976. 
250 M. C. McMaster and R. J. Madix, Surf. Sci., 1992, 275, 265-280.

251 H. P. Steinruck, A. V. Hamza and R. J. Madix, Surf. Sci., 1986, 173, L571-L575.

252 J. F. Weaver, M. A. Krzyzowski and R. J. Madix, Surf. Sci., 1997, 393, 150-161.

253 K. Golibrzuch, J. H. Baraban, P. R. Shirhatti, J. Werdecker, C. Bartels and A. M. Wodtke, Z. Phys. Chem., 2015, 229, 1929-1949.

254 B. C. Krüger, G. B. Park, S. Meyer, R. J. V. Wagner, A. M. Wodtke and T. Schäfer, Phys. Chem. Chem. Phys., 2017, 19, 19896-19903.

255 G. B. Park, B. C. Krüger, D. Borodin, T. N. Kitsopoulos and A. M. Wodtke, Rep. Prog. Phys., 2019, 82, 096401.

256 G. B. Park, T. N. Kitsopoulos, D. Borodin, K. Golibrzuch, J. Neugebohren, D. J. Auerbach, C. T. Campbell and A. M. Wodtke, Nat. Rev. Chem., 2019, 3, 723-732.

257 K. Reuter and H. Metiu, in Handbook of Materials Modeling, ed. W. Andreoni and S. Yip, Springer International, 2018, pp. 1-11.

258 M. M. Montemore, M. A. van Spronsen, R. J. Madix and C. M. Friend, Chem. Rev., 2017, 118, 2816-2862.

259 A. C. Luntz and R. D. Beck, J. Vac. Sci. Technol., A, 2017, 35, $05 \mathrm{C} 201$.

260 S. Roy, K. J. Nayanthara, N. Tiwari and A. K. Tiwari, Int. Rev. Phys. Chem., 2020, 39, 267-318.

261 H. Chadwick and R. D. Beck, Annu. Rev. Phys. Chem., 2017, 68, 39-61.

262 H. Chadwick and R. D. Beck, Chem. Soc. Rev., 2016, 45, 3576-3594.

263 A. M. Wodtke, Chem. Soc. Rev., 2016, 45, 3641-3657.

264 M. Alducin, R. Diéz Muiño and J. I. Juaristi, Prog. Surf. Sci., 2017, 92, 317-340.

265 D. Diesing and E. Hasselbrink, Chem. Soc. Rev., 2016, 45, 3747-3755.

266 S. P. Rittmeyer, V. J. Bukas and K. Reuter, Adv. Phys.: X, 2018, 3, 1381574.

267 C. Bartels, R. Cooper, D. J. Auerbach and A. M. Wodtke, Chem. Sci., 2011, 2, 1647-1655.

268 K. Golibrzuch, N. Bartels, D. J. Auerbach and A. M. Wodtke, Annu. Rev. Phys. Chem., 2015, 66, 399-425.

269 B. Jiang and H. Guo, J. Chem. Phys., 2019, 150, 180901.

270 A. Krylov, T. L. Windus, T. Barnes, E. Marin-Rimoldi, J. A. Nash, B. Pritchard, D. G. A. Smith, D. Altarawy, P. Saxe, C. Clementi, T. D. Crawford, R. J. Harrison, S. Jha, V. S. Pande and T. Head-Gordon, J. Chem. Phys., 2018, 149, 180901.

271 J. Carrasco, A. Hodgson and A. Michaelides, Nat. Mater., 2012, 11, 667-674.

272 K. Schwarz and R. Sundararaman, Surf. Sci. Rep., 2020, 75, 100492.

273 A. Groß, F. Gossenberger, X. H. Lin, M. Naderian, S. Sakong and T. Roman, J. Electrochem. Soc., 2014, 161, E3015-E3020.

274 J. J. Varghese and S. H. Mushrif, React. Chem. Eng., 2019, 4, 165-206.

275 C. Sievers, Y. Noda, L. Qi, E. M. Albuquerque, R. M. Rioux and S. L. Scott, ACS Catal., 2016, 6, 8286-8307.
276 K. Mathew, V. S. C. Kolluru, S. Mula, S. N. Steinmann and R. G. Hennig, J. Chem. Phys., 2019, 151, 234101.

277 J. A. Gauthier, S. Ringe, C. F. Dickens, A. J. Garza, A. T. Bell, M. Head-Gordon, J. K. Nørskov and K. Chan, ACS Catal., 2019, 9, 920-931.

278 S. Ringe, H. Oberhofer, C. Hille, S. Matera and K. Reuter, J. Chem. Theory Comput., 2016, 12, 4052-4066.

279 O. Andreussi and G. Fisicaro, Int. J. Quantum Chem., 2018, 119, e25725.

280 S. K. Natarajan and J. Behler, Phys. Chem. Chem. Phys., 2016, 18, 28074-28725.

281 J. Park and L. T. Roling, AIChE J., 2020, 66, e17036.

282 A. S. Nair and B. Pathak, Wiley Interdiscip. Rev.: Comput. Mol. Sci., 2020, e1508.

283 A. Groß, J. Comput. Theor. Nanosci., 2008, 5, 894-922.

284 Z. X. Luo, A. W. Castleman, Jr. and S. N. Khanna, Chem. Rev., 2016, 116, 14456-14492.

285 D. H. Z. A. H. Guo, Аnnu. Rev. Phys. Chem., 2016, 67, 135-158.

286 J. Li, B. Zhao, D. Q. Xie and H. Guo, J. Phys. Chem. Lett., 2020, 11, 8844-8860.

287 P. Hohenberg and W. Kohn, Phys. Rev., 1964, 136, B864-B871.

288 W. Kohn and L. J. Sham, Phys. Rev., 1965, 140, A1133-A1138.

289 R. Peverati and D. G. Truhlar, Philos. Trans. R. Soc., A, 2014, 372, 20120476.

290 A. D. Becke, J. Chem. Phys., 2014, 140, 18A301.

291 K. Burke, J. Chem. Phys., 2012, 136, 150901.

292 R. O. Jones, Rev. Mod. Phys., 2015, 87, 897-920.

293 H. S. Yu, S. L. Li and D. G. Truhlar, J. Chem. Phys., 2016, 145, 130901.

294 N. Q. Su and X. Xu, Annu. Rev. Phys. Chem., 2017, 68, 155-182.

295 K. Berland, V. R. Cooper, K. Lee, E. Schröder, T. Thonhauser, P. Hyldgaard and B. I. Lundqvist, Rep. Prog. Phys., 2015, 78, 066501.

296 M. Stöhr, T. Van Voorhis and A. Tkatchenko, Chem. Soc. Rev., 2019, 48, 4118-4154.

297 S. Grimme, A. Hansen, J. G. Brandenburg and C. Bannwarth, Chem. Rev., 2016, 116, 5105-5154.

298 G. P. Chen, V. K. Voora, M. M. Agee, S. G. Balasubramani and F. Furche, Annu. Rev. Phys. Chem., 2017, 68, 421-445.

299 J. P. Perdew, MRS Bull., 2013, 38, 743-750.

300 H. J. Werner, Adv. Chem. Phys., 1987, 69, 1.

301 S. Mallikarjun Sharada, T. Bligaard, A. C. Luntz, G. J. Kroes and J. K. Nørskov, J. Phys. Chem. C, 2017, 121, 19807-19815.

302 S. Mallikarjun Sharada, R. K. B. Karlsson, Y. Maimaiti, J. Voss and T. Bligaard, Phys. Rev. B, 2019, 100, 035439.

303 J. Wellendorff, T. L. Silbaugh, D. Garcia-Pintos, J. K. Nørskov, T. Bligaard, F. Studt and C. T. Campbell, Surf. Sci., 2015, 640, 36-44.

304 J. Wellendorff, K. T. Lundgaard, A. Møgelhøj, V. Petzold, D. D. Landis, J. K. Nørskov, T. Bligaard and K. W. Jacobsen, Phys. Rev. B: Condens. Matter Mater. Phys., 2012, 85, 235149.

305 J. Wellendorff, K. T. Lundgaard, K. W. Jacobsen and T. Bligaard, J. Chem. Phys., 2014, 140, 144107.

306 P. S. Schmidt and K. S. Thygesen, J. Phys. Chem. C, 2018, 122, 4381-4390. 
307 A. J. Garza, A. T. Bell and M. Head-Gordon, J. Chem. Theory Comput., 2018, 14, 3083-3090.

308 T. L. Silbaugh and C. T. Campbell, J. Phys. Chem. C, 2016, 120, 25161-25172.

309 J. L. Lansford, A. V. Mironenko and D. G. Vlachos, Nat. Commun., 2017, 8, 1842.

310 A. Erba, J. Maul, M. Ferrabone, R. Dovesi, M. Rérat and P. Carbonnière, J. Chem. Theory Comput., 2019, 15, 3766-3777.

311 S. Manzhos, M. Chan and T. Carrington, Jr., J. Chem. Phys., 2013, 139, 055101.

312 J. P. Perdew, J. A. Chevary, S. H. Vosko, K. A. Jackson, M. R. Pederson, D. J. Singh and C. Fiolhais, Phys. Rev. B: Condens. Matter Mater. Phys., 1992, 46, 6671-6687.

313 A. D. Becke, Phys. Rev. A: At., Mol., Opt. Phys., 1988, 38, 3098-3100.

314 J. P. Perdew, A. Ruzsinszky, G. I. Csonka, O. A. Vydrov, G. E. Scuseria, L. A. Constantin, X. L. Zhou and K. Burke, Phys. Rev. Lett., 2008, 100, 136406.

315 N. E. Schultz, Y. Zhao and D. G. Truhlar, J. Phys. Chem. A, 2005, 109, 11127-11143.

316 R. Peverati and D. G. Truhlar, J. Chem. Theory Comput., 2012, 8, 2310-2319.

317 H. S. Yu, W. J. Zhang, P. Verma, X. He and D. G. Truhlar, Phys. Chem. Chem. Phys., 2015, 17, 12146-12160.

318 B. G. Janesko, Top. Curr. Chem., 2015, 365, 25-52.

319 B. G. Janesko, V. Barone and E. N. Brothers, J. Chem. Theory Comput., 2013, 9, 4853-4859.

320 L. Schimka, J. Harl, A. Stroppa, A. Grüneis, M. Marsman, F. Mittendorfer and G. Kresse, Nat. Mater., 2010, 9, 741-744.

321 S. Grimme, J. Antony, S. Ehrlich and H. Krieg, J. Chem. Phys., 2010, 132, 154104.

322 S. Grimme, S. Ehrlich and K. Goerigk, J. Comput. Chem., 2011, 32, 1456-1465.

323 E. Caldeweyher, C. Bannwarth and S. Grimme, J. Chem. Phys., 2017, 147, 034112.

324 A. Tkatchenko, J. DiStasio, R. A. DiStasio, R. Car and M. Scheffler, Phys. Rev. Lett., 2012, 108, 236402.

325 A. Tkatchenko and M. Scheffler, Phys. Rev. Lett., 2009, 102, 073005.

326 M. Dion, H. Rydberg, E. Schröder, D. C. Langreth and B. I. Lundqvist, Phys. Rev. Lett., 2004, 92, 246401.

327 K. Lee, E. D. Murray, L. Z. Kong, B. I. Lundqvist and D. C. Langreth, Phys. Rev. B: Condens. Matter Mater. Phys., 2010, 82, 081101.

328 D. Chakraborty, K. Berland and T. Thonhauser, J. Chem. Theory Comput., 2020, 16, 5893-5911.

329 O. A. Vydrov and T. Van Voorhis, J. Chem. Phys., 2010, 133, 244103.

330 R. Sabatini, T. Gorni and S. de Gironcoli, Phys. Rev. B: Condens. Matter Mater. Phys., 2013, 87, 041108.

331 J. Klimes, D. R. Bowler and A. Michaelides, J. Phys.: Condens. Mater., 2010, 22, 022201.

332 J. Klimes, D. R. Bowler and A. Michaelides, Phys. Rev. B: Condens. Matter Mater. Phys., 2011, 83, 195131.

333 V. R. Cooper, Phys. Rev. B: Condens. Matter Mater. Phys., 2010, 81, 161104.
334 K. Berland and P. Hyldgaard, Phys. Rev. B: Condens. Matter Mater. Phys., 2014, 89, 035412.

335 I. Hamada, Phys. Rev. B: Condens. Matter Mater. Phys., 2015, 89, 121103.

336 G. Román-Pérez and J. M. Soler, Phys. Rev. Lett., 2009, 103, 096102.

337 L. Goerigk, J. Phys. Chem. Lett., 2015, 6, 3891-3896.

338 A. J. R. Hensley, K. Ghale, C. Rieg, T. Dang, E. Anderst, F. Studt, C. T. Campbell, J. S. McEwen and Y. Zu, J. Phys. Chem. C, 2017, 121, 4937-4945.

339 J. W. Sun, A. Ruzsinszky and J. P. Perdew, Phys. Rev. Lett., 2015, 115, 036402.

340 J. W. Sun, B. Xiao, Y. Fang, R. Haunschild, P. Hao, A. Ruzsinszky, G. I. Csonka, G. E. Scuseria and J. P. Perdew, Phys. Rev. Lett., 2013, 111, 106401.

341 L. Ferrighi, B. Hammer and G. K. H. Madsen, J. Am. Chem. Soc., 2009, 131, 10605-10609.

342 A. D. Becke and K. E. Edgecombe, J. Chem. Phys., 1990, 92, 5397-5403.

343 B. Silvi and A. Savin, Nature, 1994, 371, 683-686.

344 J. W. Sun, R. Haunschild, B. Xiao, I. W. Bulik, G. E. Scuseria and J. P. Perdew, J. Chem. Phys., 2013, 138, 044113.

345 Y. Zhao and D. G. Truhlar, J. Chem. Phys., 2006, 125, 194101.

346 R. Peverati and D. G. Truhlar, Phys. Chem. Chem. Phys., 2012, 14, 13171-13174.

347 N. Mardirossian and M. Head-Gordon, J. Chem. Phys., 2015, 142, 074111.

348 A. Stroppa, K. Termentzidis, J. Paier, G. Kresse and J. Hafner, Phys. Rev. B: Condens. Matter Mater. Phys., 2007, 76, 195440.

349 J. Paier, M. Marsman, K. Hummer, G. Kresse, I. C. Gerber and J. G. Ángyán, J. Chem. Phys., 2006, 124, 154709.

350 H. R. Liu, H. J. Xiang and X. G. Gong, J. Chem. Phys., 2011, 135, 214702.

351 N. Gerrits, E. W. F. Smeets, S. Vuckovic, A. D. Powell, K. Doblhoff-Dier and G. J. Kroes, J. Phys. Chem. Lett., 2020, 11, 10552-10560.

352 P. J. Stephens, F. J. Devlin, C. F. Chabalowski and M. J. Frisch, J. Phys. Chem., 1994, 98, 11623-11627.

353 C. T. Lee, W. T. Yang and R. G. Parr, Phys. Rev. B: Condens. Matter Mater. Phys., 1988, 37, 785-789.

354 N. Mardirossian and M. Head-Gordon, J. Chem. Phys., 2016, 144, 214110.

355 Y. Zhao and D. G. Truhlar, J. Chem. Theory Comput., 2008, 4, 1849-1868.

356 A. V. Krukau, O. A. Vydrov, A. F. Izmaylov and G. E. Scuseria, J. Chem. Phys., 2006, 125, 224106.

357 R. Peverati and D. G. Truhlar, Phys. Chem. Chem. Phys., 2012, 14, 16187-16191.

358 K. Berland, Y. Jiao, J. H. Lee, T. Rangel, J. B. Neaton and P. Hyldgaard, J. Chem. Phys., 2017, 146, 234106.

359 D. C. Langreth and J. P. Perdew, Solid State Commun., 1979, 31, 567-571.

360 D. C. Langreth and J. P. Perdew, Phys. Rev. B: Condens. Matter Mater. Phys., 1980, 21, 5469-5493.

361 F. Furche, Phys. Rev. B: Condens. Matter Mater. Phys., 2001, 64, 195120. 
362 J. Harl, L. Schimka and G. Kresse, Phys. Rev. B: Condens. Matter Mater. Phys., 2010, 81, 115126.

363 Y. Zhao, B. J. Lynch and D. G. Truhlar, J. Phys. Chem. A, 2004, 108, 4786-4791.

364 J. Paier, X. Ren, P. Rinke, G. E. Scuseria, A. Grüneis, G. Kresse and M. Scheffler, New J. Phys., 2012, 14, 043002.

365 C. Pisani, L. Maschio, S. Casassa, M. Halo, M. Schütz and D. Usvyat, J. Comput. Chem., 2008, 29, 2113-2124.

366 N. Mardirossian and M. Head-Gordon, J. Chem. Theory Comput., 2016, 12, 4303-4325.

367 T. Bligaard, R. M. Bullock, C. T. Campbell, J. G. G. Chen, B. C. Gates, R. J. Gorte, C. W. Jones, W. D. Jones, J. R. Kitchin and S. L. Scott, ACS Catal., 2016, 6, 2590-2602.

368 K. Duanmu and D. G. Truhlar, J. Chem. Theory Comput., 2016, 13, 835-842.

369 A. Gonzales-Lafont, T. N. Truong and D. G. Truhlar, J. Phys. Chem., 1991, 95, 4618-4627.

370 A. A. Viggiano, J. S. Paschkewitz, R. A. Morris and J. F. Paulson, J. Am. Chem. Soc., 1991, 113, 9404-9405.

371 Y. P. Liu, D. H. Lu, A. Gonzalez-Lafont, D. G. Truhlar and B. C. Garrett, J. Am. Chem. Soc., 1993, 115, 7806-7817.

372 G. H. Peslherbe, H. B. Wang and W. L. Hase, J. Am. Chem. Soc., 1996, 118, 2257-2266.

373 J. C. Corchado and J. Espinosa-García, J. Chem. Phys., 1996, 105, 3160-3167.

374 D. Troya and E. García-Molina, J. Phys. Chem. A, 2005, 109, 3015-3023.

375 S. Liang and A. E. Roitberg, J. Chem. Theory Comput., 2013, 9, 4470-4480.

376 G. Li, S. B. M. Bosio and W. L. Hase, J. Mol. Struct., 2000, 556, 43-57.

377 J. Pu and D. G. Truhlar, J. Chem. Phys., 2002, 116, 1468-1478.

378 A. Chakraborty, Y. Zhao, H. Lin and D. G. Truhlar, J. Chem. Phys., 2006, 124, 044315.

379 T. V. Albu and S. Swaminathan, Theor. Chem. Acc., 2007, 117, 383-395.

380 T. V. Albu and S. Swaminathan, J. Mol. Model., 2007, 13, 1109-1121.

381 N. Q. Su, J. Chen, Z. G. Sun, D. H. Zhang and X. Xu, J. Chem. Phys., 2015, 142, 084107.

382 G. K. H. Madsen, Phys. Rev. B: Condens. Matter Mater. Phys., 2007, 75, 195108.

383 J. J. Mortensen, K. Kaasbjerg, S. L. Frederiksen, J. K. Nørskov, J. P. Sethna and K. W. Jacobsen, Phys. Rev. Lett., 2005, 95, 216401.

384 J. K. Vincent, R. A. Olsen, G. J. Kroes, M. Luppi and E. J. Baerends, J. Chem. Phys., 2005, 122, 044701.

385 A. Gross, M. Scheffler, M. J. Mehl and D. A. Papaconstantopoulos, Phys. Rev. Lett., 1999, 82, 1209-1212.

386 A. Groß, A. Eichler, J. Hafner, M. J. Mehl and D. A. Papaconstantopoulos, Surf. Sci., 2003, 539, L542-L548.

387 A. Groß, A. Eichler, J. Hafner, M. J. Mehl and D. A. Papaconstantopoulos, J. Chem. Phys., 2006, 124, 174713.

388 R. E. Cohen, M. J. Mehl and D. A. Papaconstantopoulos, Phys. Rev. B: Condens. Matter Mater. Phys., 1994, 50, 14694-14697.
389 M. J. Mehl and D. A. Papaconstantopoulos, Phys. Rev. B: Condens. Matter Mater. Phys., 1996, 54, 4519-4530.

390 F. Spiegelman, N. Tarrat, J. Cuny, L. Dontot, E. Posenitsky, C. Martí, A. Simon and M. Rapacioli, Adv. Phys.: X, 2019, 5, 1710252.

391 C. Huang, M. Pavone and E. A. Carter, J. Chem. Phys., 2011, 134, 154110.

392 F. Libisch, C. Huang and E. A. Carter, Acc. Chem. Res., 2014, 47, 2768-2775.

393 C. Huang, J. Chem. Phys., 2016, 134, 154110.

394 B. O. Roos, P. R. Taylor and P. E. M. Siegbahn, Chem. Phys., 1980, 48, 157-173.

395 K. Andersson, P. Å. Malmqvist, B. O. Roos, A. J. Sadlej and K. Wolinski, J. Phys. Chem., 1990, 94, 5483-5488.

396 K. Andersson, P. A. Malmqvist and B. O. Roos, J. Chem. Phys., 1992, 96, 1218-1226.

397 J. B. Foresman, M. Head-Gordon, J. A. Pople and M. J. Frisch, J. Phys. Chem., 1992, 96, 135-149.

398 F. Libisch, J. Cheng and E. A. Carter, Z. Phys. Chem., 2013, 227, 1455-1466.

399 F. Libisch, C. Huang, P. L. Liao, M. Pavone and E. A. Carter, Phys. Rev. Lett., 2012, 109, 198303.

400 J. Cheng, F. Libisch and E. A. Carter, J. Phys. Chem. Lett., 2015, 6, 1661-1665.

401 S. Mukherjee, F. Libisch, N. Large, O. Neumann, L. V. Brown, J. Cheng, J. B. Lassiter, E. A. Carter, P. Nordlander and N. J. Halas, Nano Lett., 2012, 13, 240-247.

402 V. A. Spata and E. A. Carter, ACS Nano, 2018, 12, 3512-3522. 403 J. Martirez and E. A. Carter, Sci. Adv., 2017, 3, eaao4710.

404 J. Martirez and E. A. Carter, J. Am. Chem. Soc., 2017, 139, 4390-4398.

405 K. Morokuma, Bull. Korean Chem. Soc., 2003, 24, 797-801.

406 F. Göltl, C. Houriez, M. Guitou, G. Chambaud and P. Sautet, J. Phys. Chem. C, 2014, 118, 5374-5382.

407 J. Watts, J. Gauss and R. J. Bartlett, J. Chem. Phys., 1993, 98, 8718-8733.

408 H. J. Werner and P. J. Knowles, J. Chem. Phys., 1988, 89, 5803-5814.

409 S. R. Langhoff and E. R. Davidson, Int. J. Quantum Chem., 1974, 8, 61-72.

410 E. Bernard, C. Hoiriez, A. O. Mitruschenkov, M. Guitou and G. Chambaud, J. Chem. Phys., 2015, 142, 054703.

411 G. E. Scuseria and T. J. Lee, J. Chem. Phys., 1990, 93, 5851-5855.

412 I. Y. Zhang and A. Grüneis, Front. Mater., 2019, 6, 123.

413 T. Tsatsoulis, S. Sakong, A. Groß and A. Grüneis, J. Chem. Phys., 2018, 149, 244105.

414 J. Kolorenc and L. Mitas, Rep. Prog. Phys., 2011, 74, 026502.

415 W. M. C. Foulkes, L. Mitas, R. J. Needs and G. Rajagopal, Rev. Mod. Phys., 2001, 73, 33-83.

416 R. J. Needs, M. D. Towler, N. D. Drummond and P. López Ríos, J. Phys.: Condens. Matter, 2010, 22, 023201.

417 D. Alfè and M. J. Gillan, Phys. Rev. B: Condens. Matter Mater. Phys., 2004, 70, 161101.

418 B. M. Austin, D. Y. Zubarev and W. A. Lester, Jr., Chem. Rev., 2012, 112, 263-288. 
419 R. N. Barnett, P. J. Reynolds and W. A. Lester, Jr., J. Chem. Phys., 1985, 82, 2700-2707.

420 D. L. Diedrich and J. B. Anderson, Science, 1992, 258, 786-788.

421 J. C. Grossman and L. Mitas, Phys. Rev. Lett., 1997, 79, 4353-4356.

422 F. Fracchia, C. Filippi and C. Amovilli, J. Chem. Theory Comput., 2013, 9, 3453-3462.

423 X. J. Zhou and F. Wang, J. Comput. Chem., 2017, 38, 798-806.

424 K. Krongchon, B. Busemeyer and L. K. Wagner, J. Chem. Phys., 2017, 146, 124129.

425 P. E. Hoggan, Int. J. Quantum Chem., 2013, 113, 277-285.

426 P. E. Hoggan and A. Bouferguène, Int. J. Quantum Chem., 2014, 114, 1150-1156.

427 M. Pozzo and D. Alfè, Phys. Rev. B: Condens. Matter Mater. Phys., 2008, 78, 245313.

428 C. R. Hsing, C. M. Chang, C. Cheng and C. M. Wei, J. Phys. Chem. C, 2019, 123, 15659-15664.

429 A. Powell, G. J. Kroes and K. Doblhoff-Dier, J. Chem. Phys., 2020, 153, 224701.

430 R. O. Sharma, T. T. Rantala and P. E. Hoggan, J. Phys. Chem. C, 2020, 124, 26232-26240.

431 K. Doblhoff-Dier, G. J. Kroes and F. Libisch, Phys. Rev. B, 2018, 98, 085138.

432 J. Behler, Int. J. Quantum Chem., 2015, 115, 1032-1050.

433 J. Behler, J. Chem. Phys., 2016, 145, 170901.

434 B. Jiang, J. Li and H. Guo, J. Phys. Chem. Lett., 2020, 11, 5120-5131.

435 T. Mueller, A. Hernandez and C. H. Wang, J. Chem. Phys., 2020, 152, 050902.

436 J. Behler, Angew. Chem., Int. Ed., 2017, 56, 12828-12840.

437 N. V. Orupattur, S. H. Mushrif and V. Prasad, Comput. Mater. Sci., 2020, 174, 109474.

438 T. Kohonen, Neural Networks, 1988, 1, 3-16.

439 B. Jiang and H. Guo, J. Chem. Phys., 2014, 141, 034109.

440 B. Jiang, J. Li and H. Guo, Int. Rev. Phys. Chem., 2016, 35, 479-506.

441 J. Behler and M. Parrinello, Phys. Rev. Lett., 2007, 98, 146401.

442 J. Behler, J. Chem. Phys., 2011, 134, 074106.

443 J. Behler, J. Phys.: Condens. Matter, 2014, 136, 183001.

444 A. Brown, B. J. Braams, K. Christoffel, Z. Jin and J. M. Bowman,

J. Chem. Phys., 2003, 119, 8790-8793.

445 B. J. Braams and J. M. Bowman, Int. Rev. Phys. Chem., 2009, 28, 577-606.

446 J. M. Bowman, G. Czakó and B. N. Fu, Phys. Chem. Chem. Phys., 2011, 13, 8094-8111.

447 J. Li, K. S. Song and J. Behler, Phys. Chem. Chem. Phys., 2019, 21, 9672-9682.

448 Z. Xie and J. M. Bowman, J. Chem. Theory Comput., 2010, 6, 26-34.

449 K. J. Shao, J. Chen, Z. Q. Zhao and D. H. Zhang, J. Chem. Phys., 2016, 145, 071101.

450 T. B. Blank, S. D. Brown, A. W. Calhoun and D. J. Doren, J. Chem. Phys., 1995, 103, 4129-4137.

451 S. Lorenz, A. Groß and M. Scheffler, Chem. Phys. Lett., 2004, $395,210-215$.
452 J. Ludwig and D. G. Vlachos, J. Chem. Phys., 2007, 127, 154716.

453 J. Behler, S. Lorenz and K. Reuter, J. Chem. Phys., 2007, 127, 014705.

454 S. Manzhos and K. Yamashita, Surf. Sci., 2010, 604, 555-561.

455 T. H. Liu, B. N. Fu and D. H. Zhang, Sci. China: Chem., 2014, 57, 147-155.

456 X. J. Shen, J. Chen, Z. J. Zhang, K. J. Shao and D. H. Zhang, J. Chem. Phys., 2015, 143, 144701.

457 T. H. Liu, B. N. Fu and D. H. Zhang, J. Chem. Phys., 2018, 149, 054702.

458 S. Manzhos and T. Carrington, Jr., J. Chem. Phys., 2006, 125, 194105.

459 S. Manzhos, R. Dawes and T. Carrington, Jr., Int. J. Quantum Chem., 2015, 115, 1012-1020.

460 W. Koch and D. H. Zhang, J. Chem. Phys., 2014, 141, 021101.

461 J. S. Smith, O. Isayev and A. E. Roitberg, Chem. Sci., 2017, 8, 3192-3203.

462 M. del Cueto, X. Y. Zhou, L. S. Zhou, Y. L. Zhang, B. Jiang and H. Guo, J. Phys. Chem. C, 2020, 124, 5174-5181.

463 A. Singraber, J. Behler and C. Dellago, J. Chem. Theory Comput., 2019, 15, 1827-1840.

464 S. Plimpton, J. Comput. Phys., 1995, 117, 1-19.

465 A. Singraber, T. Morawietz, J. Behler and C. Dellago, J. Chem. Theory Comput., 2019, 15, 3075-3092.

466 Y. L. Zhang, C. Hu and B. Jiang, J. Phys. Chem. Lett., 2020, 10, 4962-4967.

467 L. J. Zhu, Y. L. Zhang, L. Zhang, X. Y. Zhou and B. Jiang, Phys. Chem. Chem. Phys., 2020, 22, 13958-13964.

468 C. Hu, Y. L. Zhang and B. Jiang, J. Phys. Chem. C, 2020, 124, 23190-23199.

469 Y. X. Zuo, C. Chen, X. G. Li, Z. Deng, Y. M. Chen, J. Behler, G. Csányi, A. V. Shapeev, A. P. Thompson, M. A. Wood and S. P. Ong, J. Phys. Chem. A, 2020, 124, 731-745.

470 S. Lee, H. W. Kim, J. Im, S. K. Kim, Y. T. Kim, H. Chang, T. W. Ko, J. Lee and Y. Hyon, J. Korean Phys. Soc., 2020, 77, 680-688.

471 Y. L. Zhang, R. J. Maurer and B. Jiang, J. Phys. Chem. C, 2020, 124, 186-195.

472 H. F. Busnengo, A. Salin and W. Dong, J. Chem. Phys., 2000, 112, 7641-7651.

473 G. Kresse, Phys. Rev. B: Condens. Matter Mater. Phys., 2000, 62, 8295-8305.

474 A. Lozano, A. Gross and H. F. Busnengo, Phys. Chem. Chem. Phys., 2009, 11, 5814-5822.

475 H. F. Busnengo, M. A. Di Césare, W. Dong and A. Salin, Phys. Rev. B: Condens. Matter Mater. Phys., 2005, 72, 125411.

476 T. Tchakoua, E. W. F. Smeets, M. Somers and G. J. Kroes, J. Phys. Chem. C, 2019, 123, 20420-20433.

477 J. Tersoff, Phys. Rev. Lett., 1986, 56, 632-635.

478 R. Moiraghi, A. Lozano, E. Peterson, A. Utz, W. Dong and H. F. Busnengo, J. Phys. Chem. Lett., 2020, 11, 2211-2218.

479 A. Lozano, X. J. Shen, R. Moiraghi, W. Dong and H. F. Busnengo, Surf. Sci., 2015, 640, 25-35.

480 Y. Xiao, W. Dong and H. F. Busnengo, J. Chem. Phys., 2010, 132, 014704. 
481 D. W. Brenner, O. A. Shenderova, J. A. Harrison, S. J. Stuart, B. Ni and S. B. Sinnott, J. Phys.: Condens. Matter, 2002, 14, 783-802.

482 J. Ischtwan and M. A. Collins, J. Chem. Phys., 1994, 100, 8080-8088.

483 K. C. Thompson and M. A. Collins, J. Chem. Soc., Faraday Trans., 1997, 93, 871-878.

484 C. Crespos, M. A. Collins, E. Pijper and G. J. Kroes, Chem. Phys. Lett., 2003, 376, 566-575.

485 C. Crespos, M. A. Collins, E. Pijper and G. J. Kroes, J. Chem. Phys., 2004, 120, 2392-2404.

486 P. N. Abufager, C. Crespos and H. F. Busnengo, Phys. Chem. Chem. Phys., 2007, 9, 2258-2265.

487 T. J. Frankcombe, M. A. Collins and D. H. Zhang, J. Chem. Phys., 2012, 137, 144701.

488 T. J. Frankcombe, Int. J. Chem. Kinet., 2018, 446-447, DOI: 10.1002/kin.21157.

489 T. J. Frankcombe, J. Chem. Phys., 2014, 446-447, 114108.

490 J. H. McCreery and G. Wolken, J. Chem. Phys., 1975, 63, 2340-2349.

491 J. H. McCreery and G. Wolken, J. Chem. Phys., 1977, 67, 2551-2559.

492 J. Q. Dai and J. Z. H. Zhang, J. Chem. Phys., 1995, 102, 6280-6289.

493 A. Forni, G. Wiesenekker, E. J. Baerends and G. F. Tantardini, J. Phys.: Condens. Matter, 1995, 7, 7195-7207.

494 L. Martin-Gondre, C. Crespos, P. Larregaray, J. C. Rayez, B. van Ootegem and D. Conte, Chem. Phys. Lett., 2009, 471, 136-142.

495 M. F. Somers, S. M. Kingma, E. Pijper, G. J. Kroes and D. Lemoine, Chem. Phys. Lett., 2002, 360, 390-399.

496 L. Martin-Gondre, C. Crespos, P. Larregaray, J. C. Rayez, D. Conte and B. van Ootegem, Chem. Phys., 2010, 367, 136-147.

497 L. Martin-Gondre, C. Crespos and P. Larregaray, Surf. Sci., 2019, 688, 45-50.

498 B. Jiang and D. Q. Xie, Sci. China: Chem., 2013, 57, 87-99. 499 B. Jiang, D. Q. Xie and H. Guo, Chem. Sci., 2013, 4, 503-508. 500 B. Jiang and H. Guo, J. Phys. Chem. C, 2013, 117, 16127-16135. 501 J. Ludwig and D. G. Vlachos, J. Chem. Phys., 2008, 128, 154708. 502 M. H. Beck, A. Jäckle, G. A. Worth and H.-D. Meyer, Phys. Rep., 2000, 324, 1-105.

503 A. Jäckle and H.-D. Meyer, J. Chem. Phys., 1996, 104, 7974-7984.

504 A. Jäckle and H.-D. Meyer, J. Chem. Phys., 1998, 109, 3772-3779.

505 C. Crespos, H. D. Meyer, R. C. Mowrey and G. J. Kroes, J. Chem. Phys., 2006, 124, 074706.

506 M. Schröder, J. Chem. Phys., 2020, 152, 024108.

507 A. Gross, S. Wilke and M. Scheffler, Phys. Rev. Lett., 1995, 75, 2718-2721.

508 G. J. Kroes, E. J. Baerends and R. C. Mowrey, Phys. Rev. Lett., 1997, 78, 3583-3586.

509 J. Q. Dai and J. C. Light, J. Chem. Phys., 1997, 107, 1676-1679.

510 A. Groß, Surf. Sci. Rep., 1998, 32, 291-340.
511 E. W. F. Smeets, G. Füchsel and G. J. Kroes, J. Phys. Chem. C, 2019, 123, 23049-23063.

512 G. A. Bocan, R. Diéz Muiño, M. Alducin, H. F. Busnengo and A. Salin, J. Chem. Phys., 2008, 128, 154704.

513 C. Díaz, J. K. Vincent, G. P. Krishnamohan, R. A. Olsen, G. J. Kroes, K. Honkala and J. K. Nørskov, Phys. Rev. Lett., 2006, 96, 096102.

514 C. Engdahl, B. I. Lundqvist, U. Nielsen and J. K. Nørskov, Phys. Rev. B: Condens. Matter Mater. Phys., 1992, 45, 11362-11365.

515 M. Hand and J. Harris, J. Chem. Phys., 1990, 92, 7610-7617. 516 A. C. Luntz and J. Harris, Surf. Sci., 1991, 258, 397-426.

517 S. Nave and B. Jackson, Phys. Rev. Lett., 2007, 98, 173003.

518 H. F. Busnengo, W. Dong and A. Salin, Phys. Rev. Lett., 2004, 93, 236103.

519 Z. S. Wang, G. R. Darling and S. Holloway, Phys. Rev. Lett., 2001, 87, 226102.

520 S. A. Adelman and J. D. Doll, J. Chem. Phys., 1976, 64, 2375-2388.

521 J. C. Tully, J. Chem. Phys., 1980, 73, 1975-1985.

522 V. J. Bukas, S. Mitra, J. Meyer and K. Reuter, J. Chem. Phys., 2015, 143, 034705.

523 Y. P. Zhou, L. S. Zhou, X. X. Hu and D. Q. Xie, J. Phys. Chem. C, 2020, 124, 10573-10583.

524 A. K. Tiwari, S. Nave and B. Jackson, J. Chem. Phys., 2010, 132, 134702.

525 A. K. Tiwari, S. Nave and B. Jackson, Phys. Rev. Lett., 2009, 103, 253201.

526 G. Henkelman and H. Jónsson, Phys. Rev. Lett., 2001, 86, 664-667.

527 H. Guo and B. Jackson, J. Chem. Phys., 2016, 144, 184709.

528 X. J. Shen, Z. J. Zhang and D. H. Zhang, Phys. Chem. Chem. Phys., 2015, 17, 25499-25504.

529 D. H. Zhang, personal communication.

530 M. Dohle and P. Saalfrank, Surf. Sci., 1997, 373, 95-108.

531 M. Wijzenbroek and M. F. Somers, J. Chem. Phys., 2012, 137, 054703.

532 A. Groß and A. Dianat, Phys. Rev. Lett., 2007, 98, 206107.

533 N. Gerrits, H. Chadwick and G. J. Kroes, J. Phys. Chem. C, 2019, 123, 24013-24023.

534 X. J. Zhou, B. Jiang and H. Guo, J. Phys. Chem. C, 2019, 123, 20893-20902.

535 N. Gerrits and G. J. Kroes, J. Phys. Chem. C, 2019, 123, 28291-28300.

536 Q. S. Wu, L. S. Zhou and H. Guo, J. Phys. Chem. C, 2019, 123, 10509-10516.

537 A. Groß, Phys. Rev. Lett., 2009, 103, 246101.

538 A. Groß, J. Chem. Phys., 2011, 135, 174707.

539 A. Groß, ChemPhysChem, 2010, 11, 1374-1381.

540 H. C. Andersen, J. Chem. Phys., 1980, 72, 2384-2393.

541 D. Novko, I. Loncaric, M. Blanco-Rey, J. I. Juaristi and M. Alducin, Phys. Rev. B, 2017, 96, 085437.

542 J. R. Manson, in Handbook of Surface Science, ed. E. Hasselbrink and B. I. Lundqvist, Elsevier, 2008, vol. 3.

543 J. R. Manson, Phys. Rev. B: Condens. Matter Mater. Phys., 1991, 43, 6924-6937.

544 J. R. Manson, Comput. Phys. Commun., 1994, 80, 145-167. 
545 M. S. Daw, S. M. Foiles and M. I. Baskes, Mater. Sci. Rep., 1993, 9, 251-310.

546 V. J. Bukas and K. Reuter, Phys. Rev. Lett., 2016, 117, 146101.

547 F. Bouakline, E. W. Fischer and P. Saalfrank, J. Chem. Phys., 2019, 150, 244105.

548 M. Timmer and P. Kratzer, Phys. Rev. B: Condens. Matter Mater. Phys., 2009, 79, 165407.

549 S. P. Rittmeyer, J. Meyer and K. Reuter, Phys. Rev. Lett., 2017, 119, 176808.

550 Y. Li and G. Wahnström, Phys. Rev. Lett., 1992, 68, 3444-3447.

551 M. Head-Gordon and J. C. Tully, J. Chem. Phys., 1995, 103, 10137-10145.

552 M. J. Puska and R. M. Nieminen, Phys. Rev. B: Condens. Matter Mater. Phys., 1983, 27, 6121-6128.

553 P. M. Echenique, R. M. Nieminen and R. H. Ritchie, Solid State Commun., 1981, 37, 779-781.

554 N. Gerrits, J. I. Juaristi and J. Meyer, Phys. Rev. B, 2020, 102, 155130.

555 M. Lindenblatt and E. Pehlke, Phys. Rev. Lett., 2006, 97, 216101.

556 A. Salin, A. Arnau, P. M. Echenique and E. Zaremba, Phys. Rev. B: Condens. Matter Mater. Phys., 1999, 59, 2537-2548.

557 S. P. Rittmeyer, J. Meyer, J. I. Juaristi and K. Reuter, Phys. Rev. Lett., 2015, 115, 046102.

558 B. Hellsing and M. Persson, Phys. Scr., 1984, 29, 360-371.

559 M. Askerka, R. J. Maurer, V. S. Batista and J. C. Tully, Phys. Rev. Lett., 2016, 116, 217601.

560 R. J. Maurer, M. Askerka, V. S. Batista and J. C. Tully, Phys. Rev. B, 2016, 94, 115432.

561 D. Novko, M. Alducin, M. Blanco-Rey and J. I. Juaristi, Phys. Rev. B, 2016, 94, 224306.

562 J. R. Trail, M. C. Graham, D. M. Bird, M. Persson and S. Holloway, Phys. Rev. Lett., 2002, 88, 166802.

563 A. C. Luntz and M. Persson, J. Chem. Phys., 2005, 123, 074704.

564 A. C. Luntz, M. Persson and G. O. Sitz, J. Chem. Phys., 2006, 124, 091101.

565 G. Füchsel, T. Klamroth, S. Monturet and P. Saalfrank, Phys. Chem. Chem. Phys., 2011, 13, 8659-8670.

566 O. Galparsoro, R. Pétuya, F. Busnengo, J. I. Juaristi, C. Crespos, M. Alducin and P. Larregaray, Phys. Chem. Chem. Phys., 2016, 18, 31378-31383.

567 E. Watts and G. O. Sitz, J. Chem. Phys., 2001, 114, 4171-4179. 568 L. C. Shackman and G. O. Sitz, J. Chem. Phys., 2005, 123, 064712. 569 J. Behler, B. Delley, S. Lorenz, K. Reuter and M. Scheffler, Phys. Rev. Lett., 2005, 94, 036104.

570 J. Behler, K. Reuter and M. Scheffler, Phys. Rev. B: Condens. Matter Mater. Phys., 2008, 77, 115421.

571 Y. H. Huang, C. T. Rettner, D. J. Auerbach and A. M. Wodtke, Science, 2000, 290, 111-114.

572 C. Carbogno, J. Behler, A. Groß and K. Reuter, Phys. Rev. Lett., 2008, 101, 096104.

573 C. Carbogno, J. Behler, K. Reuter and A. Groß, Phys. Rev. B: Condens. Matter Mater. Phys., 2010, 81, 035410.

574 J. C. Tully, J. Chem. Phys., 1990, 93, 1061-1071.

575 N. Shenvi, S. Roy and J. C. Tully, J. Chem. Phys., 2009, 130, 174107.
576 B. Gergen, H. Nienhaus, W. H. Weinberg and E. W. McFarland, Science, 2001, 294, 2521-2523.

577 J. T. Kindt, J. C. Tully, M. Head-Gordon and M. A. Gomez, J. Chem. Phys., 1998, 109, 3629-3636.

578 L. Martin-Gondre, G. A. Bocan, M. Blanco-Rey, M. Alducin, J. I. Juaristi and R. Díez Muiño, J. Phys. Chem. C, 2013, 117, 9779-9790.

579 L. Martin-Gondre, G. A. Bocan, M. Alducin, J. I. Juaristi and R. Díez Muiño, Comput. Theor. Chem., 2012, 990, 126-131.

580 O. Galparsoro, J. I. Juaristi, C. Crespos, M. Alducin and P. Larrégaray, J. Phys. Chem. C, 2017, 121, 19849-19858.

581 C. T. Rettner, H. A. Michelsen and D. J. Auerbach, Chem. Phys., 1993, 175, 157-169.

582 D. Novko, M. Blanco-Rey, J. I. Juaristi and M. Alducin, Phys. Rev. B: Condens. Matter Mater. Phys., 2015, 92, 201411.

583 J. L. Chen, X. Y. Zhou and B. Jiang, J. Chem. Phys., 2019, 150, 061101.

584 L. S. Zhou, B. Jiang, M. Alducin and H. Guo, J. Chem. Phys., 2018, 149, 031101.

585 L. S. Zhou, X. Y. Zhou, M. Alducin, L. Zhang, B. Jiang and H. Guo, J. Chem. Phys., 2018, 148, 014702.

586 X. Y. Zhou, L. Zhang and B. Jiang, J. Phys. Chem. C, 2018, 122, 15485-15493.

587 M. Karplus, R. N. Porter and R. D. Sharma, J. Chem. Phys., 1965, 43, 3259-3287.

588 R. N. Porter and L. M. Raff, in Dynamics of Molecular Collisions, Part B, ed. W. H. Miller, Plenum, New York, 1976, p. 1.

589 T. D. Sewell and D. L. Thompson, Int. J. Mod. Phys. B, 1997, 11, 1067-1112.

590 H. F. Busnengo, C. Crespos, W. Dong, J. C. Rayez and A. Salin, J. Chem. Phys., 2002, 116, 9005-9013.

591 G. J. Kroes, Prog. Surf. Sci., 1999, 60, 1-85.

592 G. J. Kroes and M. F. Somers, J. Theor. Comput. Chem., 2005, 4, 493-581.

593 C. Díaz and F. Martín, Phys. Rev. A: At., Mol., Opt. Phys., 2010, 82, 012901.

594 L. Bonnet and J. C. Rayez, Chem. Phys. Lett., 1997, 277, 183-190.

595 L. Bonnet, Int. Rev. Phys. Chem., 2013, 32, 171-228.

596 A. Rodríguez-Fernández, L. Bonnet, C. Crespos, P. Larrégaray and R. Diéz Muiño, J. Phys. Chem. Lett., 2019, 10, 7629-7635.

597 Q. H. Liu, L. Zhang, Y. Li and B. Jiang, J. Phys. Chem. Lett., 2019, 10, 7475-7481.

598 M. Mastromatteo and B. Jackson, J. Chem. Phys., 2013, 139, 194701.

599 G. R. Schoofs, C. R. Arumainayagam, M. C. McMaster and R. J. Madix, Surf. Sci., 1989, 215, 1-28.

600 B. Jiang, personal communication.

601 L. Bonnet, J. Chem. Phys., 2008, 128, 044109.

602 C. Crespos, J. Decock, P. Larrégaray and L. Bonnet, J. Phys. Chem. C, 2017, 121, 16854-16863.

603 A. Rodríguez-Fernández, L. Bonnet, C. Crespos, P. Larrégaray and R. Diéz Muiño, Phys. Chem. Chem. Phys., 2020, 22, 22805-22814.

604 X. Y. Zhou, Y. L. Zhang, H. Guo and B. Jiang, Phys. Chem. Chem. Phys., 2021, 23, 4376-4385. 
605 R. Kosloff, J. Phys. Chem., 1988, 92, 2087-2100.

606 Y. Huang, W. Zhu, D. J. Kouri and D. K. Hoffman, Chem. Phys. Lett., 1993, 206, 96-101.

607 G. J. Kroes and D. Neuhauser, J. Chem. Phys., 1996, 105, 8690-8698.

608 M. D. Feit, J. J. A. Fleck and A. Steiger, J. Comput. Phys., 1982, 47, 412-433.

609 U. Manthe, H.-D. Meyer and L. S. Cederbaum, J. Chem. Phys., 1992, 97, 3199-3213.

610 T. Wu, H.-J. Werner and U. Manthe, Science, 2004, 306, 2227-2229.

611 G. Schiffel and U. Manthe, J. Chem. Phys., 2010, 133, 174124.

612 R. v. Harrevelt and U. Manthe, J. Chem. Phys., 2004, 121, 3829-3835.

613 R. v. Harrevelt and U. Manthe, J. Chem. Phys., 2005, 123, 064106. 614 M. M. Teixidor and F. Huarte-Larrañaga, Chem. Phys., 2012, 399, 264-271.

615 P. Nieto, E. Pijper, D. Barredo, G. Laurent, R. A. Olsen, E. J. Baerends, G. J. Kroes and D. Farías, Science, 2006, 312, 86-89.

616 T. H. Liu, B. N. Fu and D. H. Zhang, Chem. Phys. Lett., 2020, 761, 138078.

617 T. H. Liu, B. N. Fu and D. H. Zhang, J. Chem. Phys., 2019, 151, 144707.

618 N. Schumacher, A. Boisen, S. Dahl, A. A. Gokhale, S. Kandoi, L. C. Grabow, J. A. Dumesic, M. Mavrikakis and I. Chorkendorff, J. Catal., 2005, 229, 265-275.

619 A. Mondal, H. Seenivasan and A. K. Tiwari, J. Chem. Phys., 2012, 137, 094708.

620 L. Zhang and B. Jiang, J. Chem. Phys., 2020, 153, 214702.

621 T. H. Liu, Z. J. Zhang, B. N. Fu, X. M. Yang and D. H. Zhang, Phys. Chem. Chem. Phys., 2016, 18, 8537-8544.

622 T. H. Liu, B. N. Fu and D. H. Zhang, Phys. Chem. Chem. Phys., 2017, 19, 11960-11967.

623 B. Jiang and H. Guo, J. Chem. Phys., 2015, 143, 164705.

624 Á. Vibók and G. G. Balint-Kurti, J. Phys. Chem., 1992, 96, 8712-8719.

625 J. Palma and D. C. Clary, J. Chem. Phys., 2000, 112, 1859-1867.

626 A. P. J. Jansen and H. Burghgraef, Surf. Sci., 1995, 344, 149-158.

627 M. N. Carré and B. Jackson, J. Chem. Phys., 1998, 108, 3722-3730.

628 Y. Xiang, J. Z. H. Zhang and D. Y. Wang, J. Chem. Phys., 2002, 117, 7698-7704.

629 S. Nave and B. Jackson, J. Chem. Phys., 2007, 127, 224702.

630 S. Nave and B. Jackson, J. Chem. Phys., 2009, 130, 054701.

631 R. Liu, H. W. Xiong and M. H. Yang, J. Phys. Chem. Lett., 2012, 3, 3776-3780.

632 R. Welsch and U. Manthe, J. Chem. Phys., 2015, 142, 064309. 633 B. Zhao and U. Manthe, J. Chem. Phys., 2019, 150, 184103. 634 B. Jackson, J. Chem. Phys., 2020, 153, 034704.

635 R. Bisson, M. Sacchi, T. T. Dang, B. Yoder, P. Maroni and R. D. Beck, J. Phys. Chem. A, 2007, 111, 12679-12683.

636 S. Habershon, D. E. Manolopoulos, T. E. Markland and T. F. Miller III, Annu. Rev. Phys. Chem., 2013, 64, 387-413.
637 Y. V. Suleimanov, F. J. Aoiz and H. Guo, J. Phys. Chem. A, 2016, 120, 8488-8502.

638 D. Chandler and P. G. Wolynes, J. Chem. Phys., 1981, 74, 4078-4095.

639 R. Welsch, K. Song, Q. Shi, S. C. Althorpe and T. F. Miller III, J. Chem. Phys., 2016, 145, 204118.

640 H. Y. Jiang, M. Kammler, F. Z. Ding, Y. Dorenkamp, F. R. Manby, A. M. Wodtke, T. F. Miller III, A. Kandratsenka and O. Bünermann, Science, 2019, 364, 379-382.

641 C. T. Rettner, H. A. Michelsen and D. J. Auerbach, J. Chem. Phys., 1995, 102, 4625-4641.

642 K. D. Rendulic, G. Anger and A. Winkler, Surf. Sci., 1989, 208, 404-424.

643 R. J. Gallagher and J. B. Fenn, J. Chem. Phys., 1974, 60, 3492-3499.

644 D. R. Killelea, PhD thesis, Tufts University, 2007.

645 H. A. Michelsen and D. J. Auerbach, J. Chem. Phys., 1991, 94, 7502-7520.

646 D. J. Auerbach, in Atomic and Molecular Beam Methods, ed. G. Scoles, Oxford University Press, New York/Oxford, 1988, vol. 1, pp. 362-379.

647 A. Hodgson, personal communication.

648 H. F. Berger, PhD thesis, Technische Universität Graz, 1992.

649 H. F. Berger, M. Leisch, A. Winkler and K. D. Rendulic, Chem. Phys. Lett., 1990, 175, 425-428.

650 G. G. Balint-Kurti, R. N. Dixon and C. C. Marston, Int. Rev. Phys. Chem., 1992, 11, 317-344.

651 R. C. Mowrey and G. J. Kroes, J. Chem. Phys., 1995, 103, 1216-1225.

652 D. Neuhauser, Chem. Phys. Lett., 1992, 200, 173-178.

653 A. Perrier, L. Bonnet, D. A. Liotard and J.-C. Rayez, Surf. Sci., 2005, 581, 189-198.

654 A. Perrier, L. Bonnet and J.-C. Rayez, J. Phys. Chem. A, 2006, 110, 1608-1617.

655 A. Perrier, L. Bonnet and J.-C. Rayez, J. Chem. Phys., 2006, 124, 194701.

656 C. Díaz, A. Perrier and G. J. Kroes, Chem. Phys. Lett., 2007, 434, 231-236.

657 O. Galparsoro, S. Kaufmann, D. J. Auerbach, A. Kandratsenka and A. M. Wodtke, Phys. Chem. Chem. Phys., 2020, 22, 17532-17539.

658 E. W. F. Smeets and G. J. Kroes, Phys. Chem. Chem. Phys., 2020, DOI: 10.1039/D1030CP05173J.

659 A. Hodgson, P. Samson, A. Wight and C. Cottrell, Phys. Rev. Lett., 1997, 78, 963-966.

660 M. J. Murphy and A. Hodgson, J. Chem. Phys., 1998, 108, 4199-4211.

661 G. Comsa and R. David, Surf. Sci., 1982, 117, 77-84.

662 J. M. Tao, J. P. Perdew, V. N. Staroverov and G. E. Scuseria, Phys. Rev. Lett., 2003, 91, 146401.

663 I. Hamada, Phys. Rev. B: Condens. Matter Mater. Phys., 2014, 89, 121103.

664 M. Bonfanti, C. Díaz, M. F. Somers and G. J. Kroes, Phys. Chem. Chem. Phys., 2011, 13, 4552-4561.

665 K. H. Chae, H. C. Lu and T. Gustafsson, Phys. Rev. B: Condens. Matter Mater. Phys., 1996, 54, 14082-14086. 
666 C. T. Rettner and D. J. Auerbach, J. Chem. Phys., 1996, 104, 2732-2739.

667 G. Anger, A. Winkler and K. D. Rendulic, Surf. Sci., 1989, 220, 1-17.

668 A. C. Luntz, J. K. Brown and M. D. Williams, J. Chem. Phys., 1990, 93, 5240-5246.

669 J. P. Perdew, Phys. Rev. B: Condens. Matter Mater. Phys., 1986, 33, 8822-8824.

670 M. del Cueto, R. J. Maurer, A. Al Taleb, D. Farías, F. Martín and C. Díaz, J. Phys.: Condens. Matter, 2019, 31, 135901.

671 P. Samson, A. Nesbitt, B. E. Koel and A. Hodgson, J. Chem. Phys., 1998, 109, 3255-3264.

672 B. Hammer, Phys. Rev. Lett., 1999, 83, 3681-3684.

673 J. Werdecker, B. J. Chen, M. E. van Reijzen, A. Farjamnia, B. Jackson and R. D. Beck, Phys. Rev. Res., 2020, 2, 043251.

674 P. Nieto, D. Barredo, D. Farías and R. Miranda, J. Phys. Chem. A, 2011, 115, 7283-7290.

675 C. Resch, V. Zhukov, A. Lugstein, H. F. Berger, A. Winkler and K. D. Rendulic, Chem. Phys., 1993, 177, 421-431.

676 H. Mortensen, E. Jensen, L. Diekhöner, A. Baurichter, A. C. Luntz and V. V. Petrunin, J. Chem. Phys., 2003, 118, 11200-11209.

677 C. Cottrell, R. N. Carter, A. Nesbitt, P. Samson and A. Hodgson, J. Chem. Phys., 1997, 106, 4714-4722.

678 C. Resch, H. F. Berger, K. D. Rendulic and E. Bertel, Surf. Sci., 1994, 316, L1105-L1109.

679 M. Beutl, M. Riedler and K. D. Rendulic, Chem. Phys. Lett., 1995, 247, 249-252.

680 M. Beutl, J. Lesnik, K. D. Rendulic, R. Hirschl, A. Eichler, G. Kresse and J. Hafner, Chem. Phys. Lett., 2001, 342, 473-478.

681 J. Lesnik, PhD thesis, Technische Universität Graz, 2001.

682 M. Gostein and G. O. Sitz, J. Chem. Phys., 1997, 106, 7378-7390.

683 M. A. Di Césare, H. F. Busnengo, W. Dong and A. Salin, J. Chem. Phys., 2003, 118, 11226-11234.

684 I. M. N. Groot, J. C. Juanes-Marcos, C. Díaz, M. F. Somers, R. A. Olsen and G. J. Kroes, Phys. Chem. Chem. Phys., 2010, 12, 1331-1340.

685 H. Ueta, I. M. N. Groot, M. A. Gleeson, S. Stolte, G. C. McBane, L. B. F. Juurlink and A. W. Kleyn, ChemPhysChem, 2008, 9, 2372-2378.

686 T. F. Hanisco and A. C. Kummel, J. Vac. Sci. Technol., A, 1993, 11, 1907-1913.

687 C. T. Rettner, H. Stein and E. K. Schweizer, J. Chem. Phys., 1988, 89, 3337-3341.

688 C. T. Rettner, E. K. Schweizer, H. Stein and D. J. Auerbach, Phys. Rev. Lett., 1988, 61, 986-989.

689 B. J. Lynch, P. L. Fast, M. Harris and D. G. Truhlar, J. Phys. Chem. A, 2000, 104, 4811-4815.

690 A. D. Becke, J. Chem. Phys., 1996, 104, 1040-1046.

691 J. Paier, M. Marsman and G. Kresse, J. Chem. Phys., 2007, 127, 024103.

692 J. Heyd, G. E. Scuseria and M. Ernzerhof, J. Chem. Phys., 2003, 118, 8207-8215.

693 J. Heyd, G. E. Scuseria and M. Ernzerhof, J. Chem. Phys., 2006, 124, 219906.
694 C. Mosch, C. Koukounas, N. Bacalis, A. Metropoulos, A. Gross and A. Mavridis, J. Phys. Chem. C, 2008, 112, 6924-6932.

695 N. Gerrits, J. Geweke, E. W. F. Smeets, J. Voss, A. M. Wodtke and G. J. Kroes, J. Phys. Chem. C, 2020, 124, 15944-15960.

696 J. P. Perdew, M. Ernzerhof and K. Burke, J. Chem. Phys., 1996, 105, 9982-9985.

697 B. Hammer and J. K. Nørskov, Nature, 1995, 376, 238-240.

698 B. Hammer and J. K. Nørskov, Surf. Sci., 1995, 343, 211.

699 S. L. Tait, Z. Dohnáleck, C. T. Campbell and B. D. Kay, Surf. Sci., 2005, 591, 90-107.

700 R. D. Levine, Molecular Reaction Dynamics, Cambridge University Press, Cambridge, 2005.

701 R. A. Marcus, J. Chem. Phys., 1966, 45, 4493-4499.

702 E. A. McCullough and R. E. Wyatt, J. Chem. Phys., 1969, 51, 1253-1254.

703 F. Abild-Petersen, O. Lytken, J. Engbæk, G. Nielsen, I. Chorkendorff and J. K. Nørskov, Surf. Sci., 2005, 590, 127-137.

704 R. C. Egeberg, S. Ullmann, I. Alstrup, C. B. Mullins and I. Chorkendorff, Surf. Sci., 2002, 497, 183-193.

705 T. P. Beebe, D. W. Goodman, B. D. Kay and J. T. Yates, J. Chem. Phys., 1987, 87, 2305-2315.

706 D. R. Palo, R. A. Dagle and J. D. Holladay, Chem. Rev., 2007, 107, 3992-4021.

707 J. P. Perdew, R. G. Parr, M. Levy and J. L. Balduz, Phys. Rev. Lett., 1982, 49, 1691-1694.

708 E. Livshits, R. Baer and R. Kosloff, J. Phys. Chem. A, 2009, 113, 7521-7527.

709 A. Hellman, B. Razaznejad and B. I. Lundqvist, Phys. Rev. B: Condens. Matter Mater. Phys., 2005, 71, 205424.

710 Y. Zhang and W. Yang, J. Chem. Phys., 1998, 108, 2604-2608.

711 A. J. Cohen, P. Mori-Sánchez and W. T. Yang, Science, 2008, 321, 792-794.

712 C. Li, X. Zheng, N. Q. Su and W. T. Yang, Natl. Sci. Rev., 2018, 5, 203-215.

713 Y. K. Zhang and W. T. Yang, J. Chem. Phys., 1998, 109, 2604-2608.

714 A. J. Cohen, P. Mori-Sánchez and W. T. Yang, Chem. Rev., 2012, 112, 289-320.

715 A. Salin, J. Chem. Phys., 2006, 124, 104704.

716 A. Patra, J. W. Sun and J. P. Perdew, Phys. Rev. B, 2019, 035442.

717 K. T. Lundgaard, J. Wellendorff, J. Voss, K. W. Jacobsen and T. Bligaard, Phys. Rev. B, 2016, 93, 235162.

718 J. Q. Dai and J. C. Light, J. Chem. Phys., 1998, 108, 7816-7819.

719 B. Hammer, M. Scheffler, K. W. Jacobsen and J. K. Nørskov, Phys. Rev. Lett., 1994, 73, 1400-1403.

720 U. Manthe, J. Chem. Phys., 2015, 142, 244109.

721 U. Manthe, J. Condens. Matter Phys., 2017, 29, 253001.

722 D. Schapers, B. Zhao and U. Manthe, Chem. Phys., 2018, 509, 37-44.

723 U. Manthe, Chem. Phys., 2018, 515, 279-286.

724 B. C. Krüger, N. Bartels, C. Bartels, A. Kandratsenka, J. C. Tully, A. M. Wodtke and T. Schafer, J. Phys. Chem. C, 2015, 119, 3268-3272.

725 C. L. Box, Y. L. Zhang, R. R. Yin, B. Jiang and R. J. Maurer, J. Am. Chem. Soc., 2020, DOI: 10.1021/jacsau.1020c00066. 
726 R. Armiento and A. E. Mattsson, Phys. Rev. B: Condens. Matter Mater. Phys., 2005, 72, 085108.

727 L. Hedin, Phys. Rev., 1965, 139, A796-A823.

728 F. Abild-Petersen and M. P. Andersson, Surf. Sci., 2007, 601, 1747-1753.

729 H. Guo and B. Jackson, J. Phys. Chem. C, 2015, 119, 14769-14779.

730 H. F. Berger and K. D. Rendulic, Surf. Sci., 1991, 253, 325-333.

731 D. J. Auerbach, personal communication.

732 I. M. N. Groot, A. W. Kleyn and L. B. F. Juurlink, Angew. Chem., Int. Ed., 2011, 50, 5174-5177.

733 D. C. Seets, C. T. Reeves, B. A. Ferguson, M. C. Wheeler and C. B. Mullins, J. Chem. Phys., 1997, 107, 10229-10241.
734 G. N. Derry, M. E. Kern and E. H. Worth, J. Vac. Sci. Technol., A, 2015, 33, 060801.

735 P. J. Feibelman, Phys. Rev. B: Condens. Matter Mater. Phys., 1995, 52, 16845-16854.

736 NIST, Computational Chemistry Comparison and Benchmark DataBase Release 20, https://cccbdb.nist.gov/elecaff1x.asp.

737 A. Ramírez-Solís, J. Vigué, G. Hinojosa and H. Saint-Martin, Phys. Rev. Lett., 2020, 124, 056001.

738 K. Yang, J. J. Zheng, Y. Zhao and D. G. Truhlar, J. Chem. Phys., 2010, 132, 164117.

739 H. S. Yu, X. He, S. L. Li and D. G. Truhlar, Chem. Sci., 2016, 7, 5032-5051.

740 D. Mahlberg, S. Sakong, K. Forster-Tonigold and A. Groß, J. Chem. Theory Comput., 2019, 15, 3250-3259. 